\title{
Intestinal and systemic cytotoxic T lymphocyte and humoral immune responses to oral and parenteral reovirus infection
}

Jonathan Reid Fulton

West Virginia University

Follow this and additional works at: https://researchrepository.wvu.edu/etd

\section{Recommended Citation}

Fulton, Jonathan Reid, "Intestinal and systemic cytotoxic T lymphocyte and humoral immune responses to oral and parenteral reovirus infection" (2006). Graduate Theses, Dissertations, and Problem Reports. 2398.

https://researchrepository.wvu.edu/etd/2398

This Dissertation is protected by copyright and/or related rights. It has been brought to you by the The Research Repository @ WVU with permission from the rights-holder(s). You are free to use this Dissertation in any way that is permitted by the copyright and related rights legislation that applies to your use. For other uses you must obtain permission from the rights-holder(s) directly, unless additional rights are indicated by a Creative Commons license in the record and/ or on the work itself. This Dissertation has been accepted for inclusion in WVU Graduate Theses, Dissertations, and Problem Reports collection by an authorized administrator of The Research Repository @ WVU.

For more information, please contact researchrepository@mail.wvu.edu. 
Intestinal and Systemic Cytotoxic T Lymphocyte and Humoral

Immune Responses to Oral and Parenteral Reovirus Infection

\author{
Jonathan Reid Fulton
}

\begin{abstract}
A Dissertation Submitted to
The School of Medicine at

West Virginia University in partial fulfillment

of the requirements for the degree of
\end{abstract}

Doctor of Philosophy

in

Microbiology and Immunology

Christopher F. Cuff, Ph.D. Chair

James M. Sheil, Ph.D.

Albert S. Berrebi, Ph.D.

Douglas L. Drevets, M.D.

Solveig G. Ericson, M.D., Ph.D.

Department of Microbiology and Immunology

Morgantown, WV

2006

Keywords: Reovirus, Cytotoxic T Lymphocytes, Intestinal Immunity, Aging 


\begin{abstract}
Intestinal and Systemic Cytotoxic T Lymphocyte and Humoral Immune Responses to Oral and Parenteral Reovirus Infection
\end{abstract}

Jonathan Reid Fulton

This work was undertaken to determine effects of routes of infection with reovirus induction of responder cytotoxic T lymphocytes (CTL). Further, this work concerns differential effects of age on humoral and CTL responses following oral and parenteral reovirus infection. Despite considerable knowledge about non-intestinal systemic viral immunity, intestinal immune response to viral infection is less understood, and it remains unknown what differences, may occur in CTL populations induced by local viral infections in the intestine or systemic periphery.

We infected mice orally or in hind footpads with reovirus, serotype 1, strain Lang (TIL), and utilized flow cytometry to assess $\mathrm{T}$ cell receptor (TCR) V $\beta$ repertoire of $\mathrm{CD} 8^{+}$cells in draining lymphoid tissues and spleens for reovirus-driven proliferative changes. We observed predominant expansion of $\mathrm{V} 6^{+} \mathrm{CD} 8^{+} \mathrm{CTL}$ in spleens and Peyer's patches (PP) of orally infected mice, as well as spleens and popliteal lymph nodes of footpad infected mice. $\mathrm{V} \beta 6^{+}$ $\mathrm{CD} 8^{+}$cells from orally and footpad infected mice mediated reovirus-specific cytotoxicity. TCR $\beta$ chain complementarity determining region $3 \beta$ (CDR $3 \beta$ length profile analysis of $\mathrm{V} \beta 6^{+} \mathrm{CD} 8^{+}$cell lines from orally and footpad infected mice, as well as cells recovered from adoptive transfer into reovirus infected SCID recipients, showed a consistent, clear, and uniform expansion of one or few clones bearing identical CDR3 $\beta$ length, indicating that CTL responses following oral or parenteral infection are likely dominated by identical CTL populations.

We also compared the ability of old and young mice to mount CTL and humoral responses to reovirus. We found old and young mice had similar CTL frequency and cytotoxicity following oral and parenteral infection. We observed that while old mice mounted $\operatorname{IgG}$ responses to parenteral reovirus equivalent to young mice, $\operatorname{IgA}$ responses of orally infected aged mice were elevated compared to young mice. These data indicate old mice retain the ability to mount CTL and humoral responses against reovirus in the intestine and systemic periphery. Furthermore, old mice have potentiation of intestinal IgA responses compared to young following intestinal infection. Our results suggest the intestine can be utilized as a route of immunization to provide efficacious cell-mediated and humoral immunity in the aged. 


\section{Table of Contents}

Intestinal and Systemic Cytotoxic T Lymphocyte and Humoral Immune Responses to Oral and

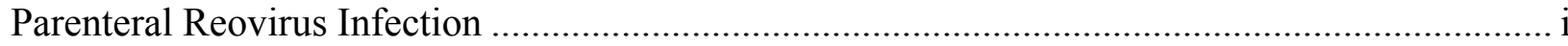

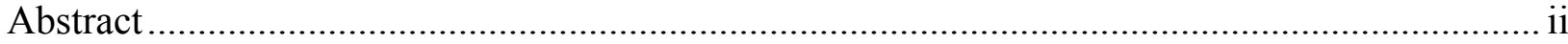

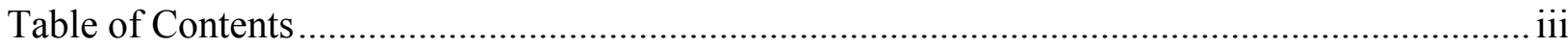

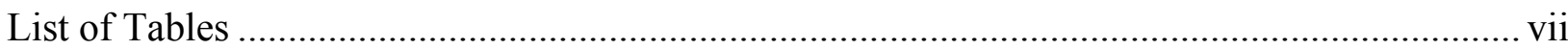

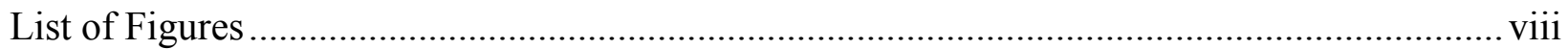

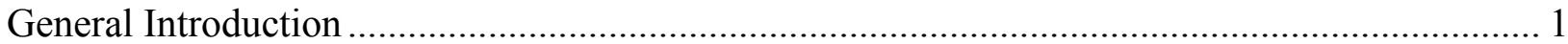

Gastrointestinal immunity: passive or active, innate or adaptive....................................... 2

Sleuth of the sluice; sentinel tissues and immune priming sites that are the Peyer's patches.

Intestinal T cells are often of uncertain origin and function Vis a Vis peripheral T cells.... 6

$\mathrm{T}$ cells of the intestinal mucosa are strikingly oligoclonal, by comparison with those of the

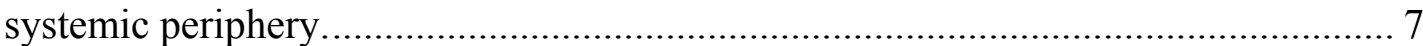

Antigen-specific cytotoxicity is mediated by intestinal IEL of conventional CD8 $\alpha \beta^{+}$

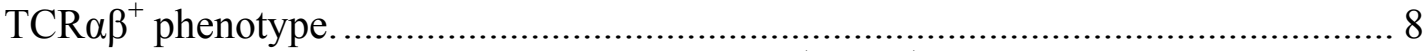

Functions of intestinal IEL with unconventional $\mathrm{CD}^{+} \mathrm{TCR}^{+}$phenotypes........................ 9

Impact of unique intestinal $\mathrm{CD}^{+}$responder populations primed in situ. ......................... 11

Reovirus as a model intestinal and systemic pathogen ....................................................... 12

Reovirus, model infectious organism for the elucidation of mucosal and systemic immune

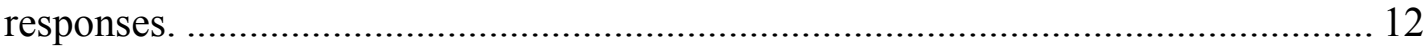

Natural reovirus T1L infection causes ileal disease .................................................... 13

Reovirus elicits innate immune responses............................................................... 15

Reovirus elicits adaptive immune responses................................................................ 16

The phenotype and function of reovirus-specific CTL .................................................. 17

$\mathrm{CD}^{+} \mathrm{CTL}$ play a role in clearing reovirus infection, but are not necessarily a sine qua non of effective anti-reovirus immunity.................................................................... 19

Reovirus-specific lymphocytes protect against reovirus-induced pathology..................... 20

Reovirus-specific CTL help protect against visceral organ disease................................... 21

Conventional and unconventional CTL are induced in respiratory tract associated lymphoid tissue following infection of the respiratory tract with reovirus............... 21

The $\mathrm{T}$ cell receptor; rearrangement, selection, and function ................................................ 22

Rearrangement of the TCR gene segments during T cell ontogeny; specificity through

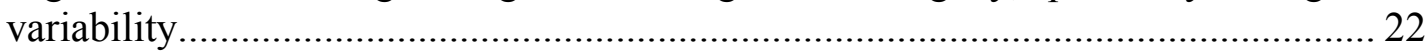

Germ-line polymorphism governs the potential of the TCR V $\beta$ repertoire. ...................... 25

Intra-thymic post-rearrangement negative selection of the TCR repertoire congenital

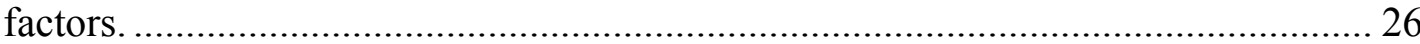

Extra-thymic modification of the TCR repertoire of the mature peripheral T cell pool....27

Positive and negative selection of mature peripheral $\mathrm{T}$ cells by superantigens. ................ 27

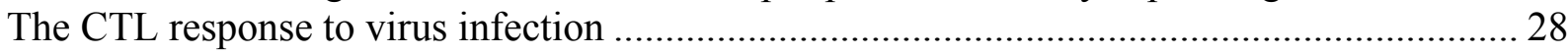

General characteristics of viral infection and the innate host immune response. .............. 28

CTL and NK cells utilize similar mechanisms to mediate cellular cytotoxicity................ 29

Innate immunity to viral infection both presages and precipitates adaptive $\mathrm{T}$ cell

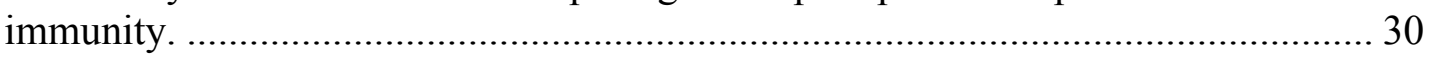

Dendritic cells are the premier antigen presenting cells in priming a CTL response. ....... 31 
Generation and presentation of viral peptide epitopes.

Ligation of co-stimulatory molecules on APC by responder T cell receptors potentiates, augments, and maintains $\mathrm{T}$ cell responses.

$\mathrm{CD}^{+} \mathrm{T}$ cells are induced to proliferate, emigrate and differentiate after TCR ligation of cognate antigen and restricting MHC molecules.

Kinetics of the CTL response are similar for several viral infections; prima facie and heuristic evidence that proliferative characteristics of anti-viral CTL responses are conserved.

Quantification by LDA of CTL response magnitude, and cotemporaneous generation of

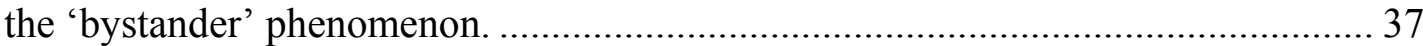

Reevaluation of quantification by LDA of CTL response magnitude, and cotemporaneous reassessment of the 'bystander' phenomenon. ..................................................... 38

Quantification of CTL response magnitude by IFN- $\gamma$ ELISPOT, intracellular cytokine staining, and $\mathrm{MHC}$ class I/peptide tetramers staining.............................................. 40

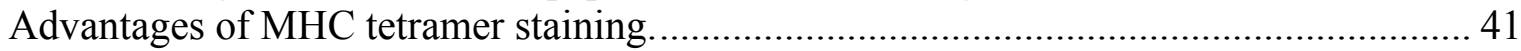

Advantages of intracellular interferon- $\gamma$ staining............................................................. 42

Decline of the CTL response with resolution of infection, maintenance of memory and

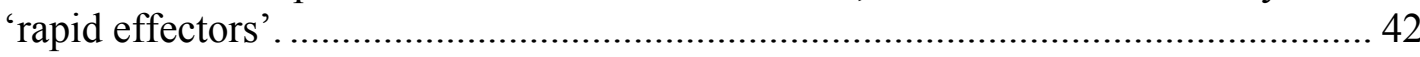

Loss of CTL with continuous antigenic stimulation can be limited by anti-apoptotic and T

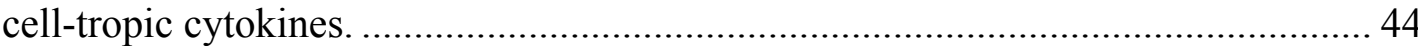

Anti-viral CTL can be generated against epitopes derived from numerous viral proteins. 46

Anti-viral CTL responses are usually, but not exclusively, generated against a panel of distinct epitopes.

Differential influences of the intestinal and systemic peripheral immune tissues upon cellular

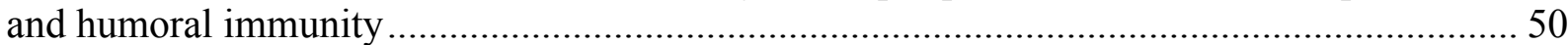

Issues of integrin-addressin mediated lymphocyte trafficking, and 'common mucosal

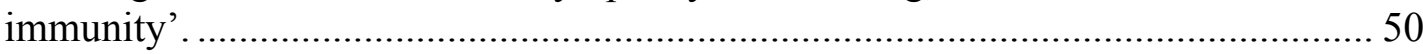

Expression of cell surface integrin molecules direct subsets of activated lymphocytes to the mucosa..................................................................................................... 52

Immune responses are not necessarily confined to either the systemic or mucosal immune

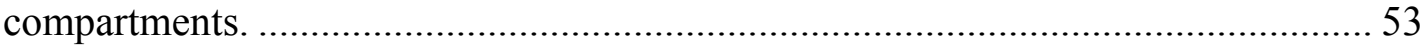

Differences in the systemic and mucosal humoral immune responses may reflect underlying $\mathrm{T}$ helper $1 / \mathrm{T}$ helper 2 or other microenvironmental differences............. 55

Dendritic cells at different anatomic locations are functionally distinct as regards $\mathrm{T}$ cell priming and differentiation, as evidenced by $\mathrm{T}$ helper cell cytokine profiles........... 58

Dendritic cells are also capable of unconventional epitope processing and presentation. . 60

Dendritic cells at different anatomic locations may be functionally distinct as regards epitope processing and presentation; a mechanism of possible tissue-specific $\mathrm{T}$ cell priming as a function of microenvironment........................................................... 61

Tissue-specific modification of an extant, ongoing CTL response may reshape the final TCR repertoire. 62

Evidence for continuous reshaping of the $\mathrm{T}$ cell repertoire in the intestinal mucosa............... 63

Differential reshaping of the virus-specific CTL repertoire as a function of distinct

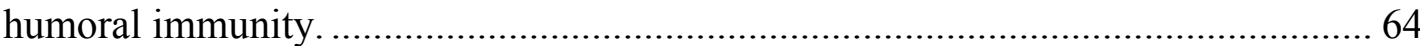

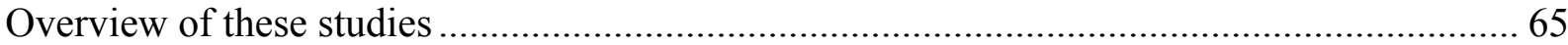

The CTL V $\beta$ Repertoire Induced Following Oral or Systemic Infection with Reovirus... 66 


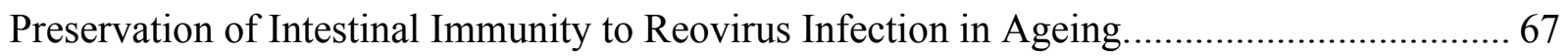

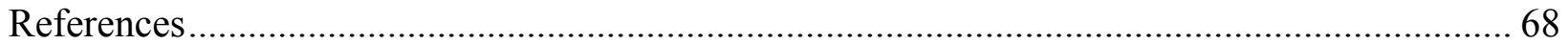

The CTL V $\beta$ Repertoire Induced Following Oral or Systemic Infection with Reovirus ........... 116

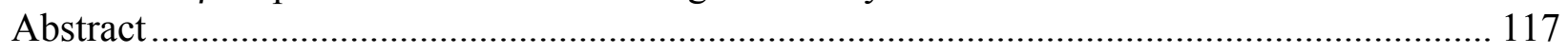

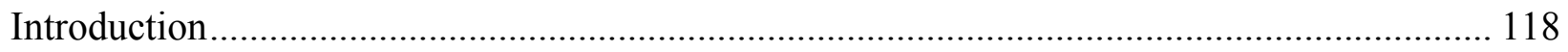

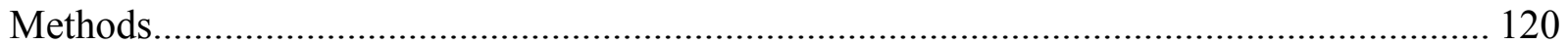

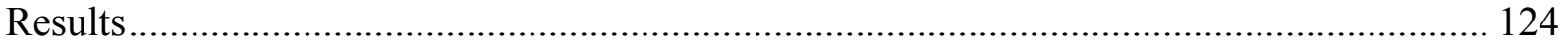

Reovirus T1L infection alters the $\mathrm{V} \beta$ repertoire of $\mathrm{CD}^{+} \mathrm{T}$ cells in the spleen............. 124

In vitro re-stimulation with reovirus also expands virus-specific $\mathrm{V} \beta 6^{+} \mathrm{CD} 8^{+} \mathrm{T}$ cells from the spleens of hind footpad infected mice..................................................... 125

Effect of oral and hind footpad infection with reovirus T1L on the V $\beta$ repertoire of CD8 ${ }^{+}$

cells in local lymphoid tissue. ...................................................................... 125

$\mathrm{V} \beta$ repertoire of reovirus-specific CTL in PP and PLN ......................................... 126

$\mathrm{V} \beta 6^{+} \mathrm{CD}^{+}$cells mediate reovirus-specific cytotoxicity. ........................................... 126

Adoptively transferred, reovirus-primed $\mathrm{CD}^{+}$cells from immunocompetent donors expand in reovirus-infected SCID recipients. .................................................. 127

$\mathrm{CD}^{+} \mathrm{T}$ cells recovered from the spleens of infected SCID recipients of immune splenocytes are enriched for virus-specific CTLs ........................................... 128

Adoptively transferred PP lymphocytes from reovirus immune donors, but not those from naïve donors, are enriched for virus-specific CTLs in reovirus-infected SCID

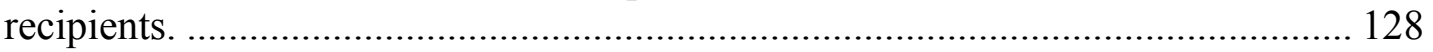

In vitro re-stimulation results in the continued expansion of adoptively transferred immune $\mathrm{V} 6^{+} \mathrm{CD}^{+} \mathrm{PP}$ lymphocytes recovered from the spleens of reovirus infected SCID recipients.

Effect of oral and hind footpad infection with reovirus T1L on the CDR3 $\beta$ length profile of $\mathrm{CD}^{+}$cells in local lymphoid tissue and spleen.

Adoptively transferred, reovirus-primed $\mathrm{V} \beta 6^{+}$, but not $\mathrm{V} \beta 2^{+}, \mathrm{CD} 8^{+}$cells from reovirusimmune donors show altered CDR3 $\beta$ length profiles upon recovery from reovirusinfected SCID recipients.

In vitro re-stimulation with reovirus alters the $\mathrm{CDR} 3 \beta$ length profiles $\mathrm{V} \beta 6^{+} \mathrm{CD} 8^{+} \mathrm{T}$ cells from the spleens of orally infected and footpad infected mice. ............................ 130

In vitro re-stimulation with reovirus further alters the CDR3 $\beta$ length profiles $\mathrm{V} \beta 6^{+} \mathrm{CD} 8^{+} \mathrm{T}$ cells recovered from the spleens of infected SCID recipients of orally infected and

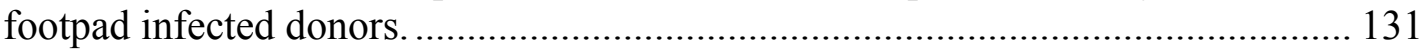

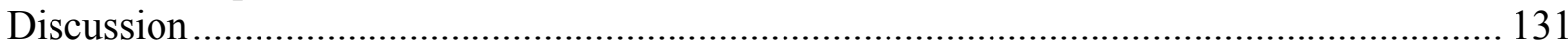

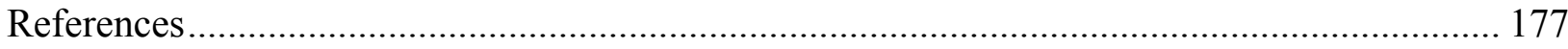

Preservation of Intestinal Immunity to Reovirus Infection in Ageing.................................. 185

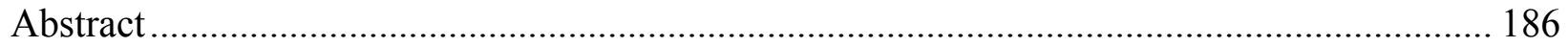

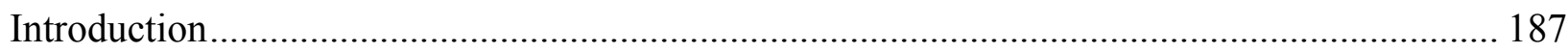

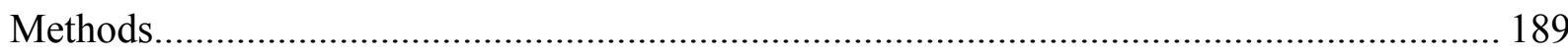

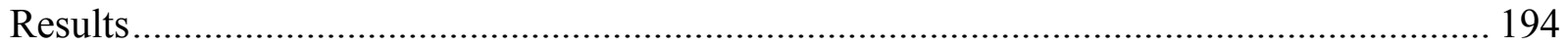

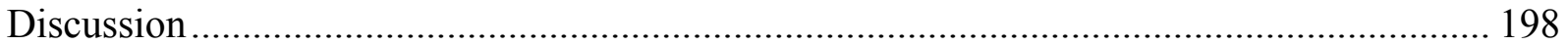

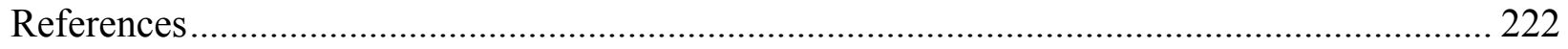

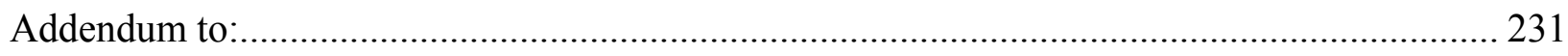

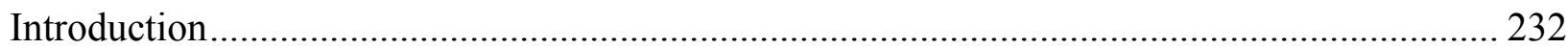

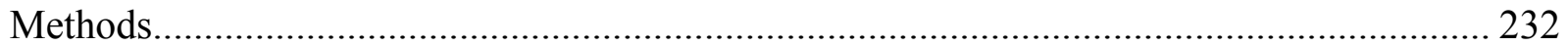




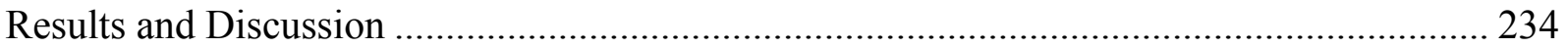

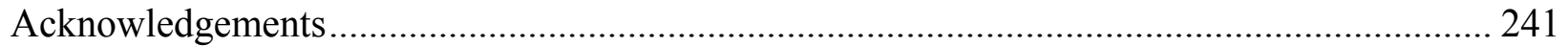

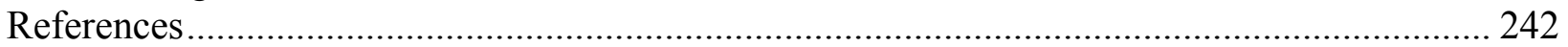

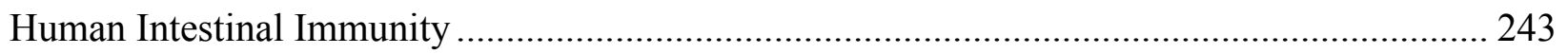

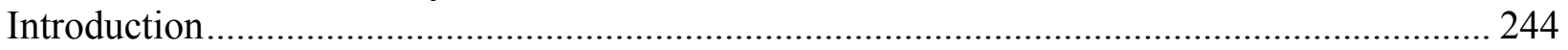

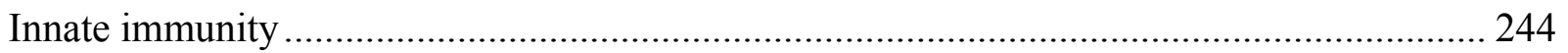

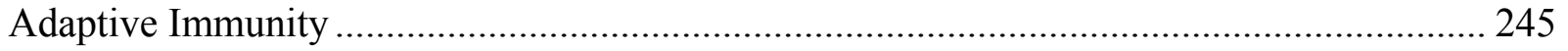

Organization and Location of Intestinal Lymphocytes: ............................................ 245

Cellular Immunity ......................................................................................... 248

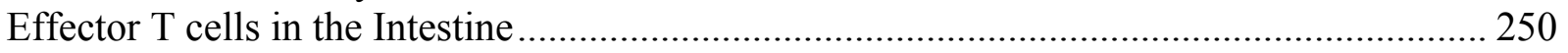

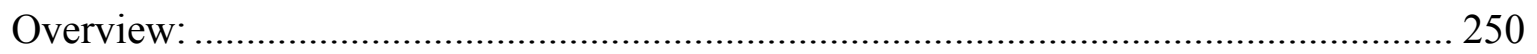

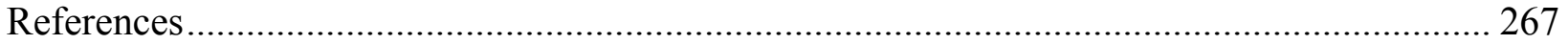

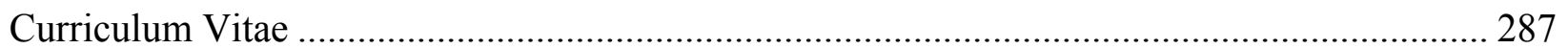




\section{List of Tables}

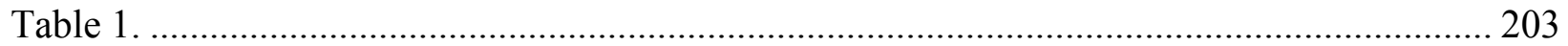

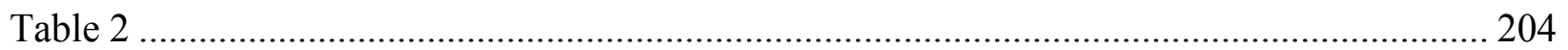

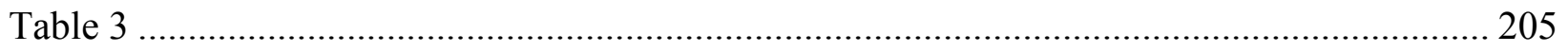




\section{List of Figures}

Figure 1. V $\beta$ repertoire of $\mathrm{CD} 8^{+} \mathrm{T}$ cells from the Peyer's patches of $\mathrm{C} 3 \mathrm{H}$ mice infected perorally and the popliteal lymph nodes of mice infected in the hind footpads with reovirus 7 days previously (A and B respectively), compared to Peyer's patch and popliteal 1 Data are expressed as mean percentage \pm standard deviations.

Figure 2. $\mathrm{V} \beta$ repertoire of representative cultures of $\mathrm{CD} 8^{+}$Peyer's patches from $\mathrm{C} 3 \mathrm{H}$ mice infected perorally and popliteal lymph nodes from mice infected in the hind footpads with reovirus (A and $\mathrm{B}$ respectively). Shown are the percentages of $\mathrm{CD}^{+}$cells staining positive with a panel of FITC-anti-V $\beta$ antibodies immediately ex vivo and after 2 weeks of in vitro restimulation with reovirus. In both cultures there has been an expansion of the $\mathrm{V} \beta 6^{+} \mathrm{CD} 8^{+}$ subpopulation, with $\mathrm{V} \beta 8.1,2^{+}$and $\mathrm{V} \beta 8.3^{+}$also showing increased representation in Peyer's patch cultures. Data is show as mean percentage expression.

Figure $3 .{ }^{51} \mathrm{Cr}$ release assay of Peyer's patch and popliteal lymph node cells derived from $\mathrm{C} 3 \mathrm{H}$ mice infected perorally or in the hind footpads and restimulated with reovirus in vitro for a period of 2 weeks. Data is given as mean percent specific lysis \pm standard deviation...... 144

Figure 4. Ten days following peroral or hind footpad infection of $\mathrm{C} 3 \mathrm{H}$ mice with reovirus, splenocytes were assessed by flow cytometry for $\mathrm{V} \beta$ repertoire expression among $\mathrm{CD} 8^{+} \mathrm{T}$ cells. (A.) The percentage of $\mathrm{CD} 8^{+}$cells staining positive with each anti-V $\beta$ antibody were determined for infected and non-infected control mice. Data are given as mean percentage \pm standard deviation. For all three groups, $\mathrm{n}=9$.

Figure 5. V $\beta$ repertoire of cultured $\mathrm{CD} 8^{+}$splenocytes from $\mathrm{C} 3 \mathrm{H}$ mice infected perorally with reovirus. Shown are selected $\mathrm{V} \beta$ staining profiles of $\mathrm{CD} 8^{+}$splenocytes (A) and after 5 weeks in culture (B). Restimulation in vitro with reovirus during culture has selectively expanded the $\mathrm{V} \beta 6^{+} \mathrm{CD} 8^{+}$subpopulation, with conspicuous representation of the $\mathrm{V} \beta 7^{+}$, $\mathrm{V} \beta 8.1,2^{+}$and $\mathrm{V} \beta 8.3^{+}$and $\mathrm{V} \beta 14^{+}$subpopulations after 5 weeks.

Figure $6 .{ }^{51} \mathrm{Cr}$ release assay of splenocytes derived from $\mathrm{C} 3 \mathrm{H}$ mice previously infected perorally with reovirus. Splenocytes were restimulated in vitro for a period of 5 weeks prior to analysis. Data is given as mean lysis.

Figure 7. Figure 7. $\mathrm{V} \beta$ repertoire of $\mathrm{CD}^{+}$splenocytes from $\mathrm{C} 3 \mathrm{H}$ mice infected with reovirus in the hind footpads. Shown are selected $\mathrm{V} \beta$ staining profiles of $\mathrm{CD} 8^{+}$splenocytes after 2 weeks of culture (A) and after 5 weeks of culture (B). Re-stimulation with reovirus in vitro has selectively expanded the $\mathrm{V} \beta 6^{+} \mathrm{CD}^{+}$subpopulation, and to a lesser extent the $\mathrm{V} \beta 7$ and $\mathrm{V} \beta 8.1$ subpopulations, from weeks 2 to 5 .

Figure 8. Flow cytometric analysis of the $\mathrm{V} \beta 6, \mathrm{~V} \beta 7$, and $\mathrm{V} \beta 8$ expression of fractions of sorted $\mathrm{CD}^{+}$splenocytes from footpad-infected $\mathrm{C} 3 \mathrm{H}$ mice. Splenocytes were cultured for 3 weeks and complement depleted of $\mathrm{CD} 4^{+}$cells as described in methods. $\mathrm{CD} 8^{+}$cells were then sorted into $\mathrm{V} \beta 6^{+}, \mathrm{V} \beta 7^{+}$, and $\mathrm{V} \beta 8^{+}$enriched and depleted fractions by indirect panning... 154

Figure 9. Representative ${ }^{51} \mathrm{Cr}$ release assays of virus-specific cytotoxicity mediated by selected $\mathrm{V} \beta$ subpopulations of cultured $\mathrm{CD}^{+}$splenocytes from $\mathrm{C} 3 \mathrm{H}$ mice previously infected in the hind footpads or perorally with reovirus. Splenocytes from footpad and perorally infected mice were cultured for 3 weeks and then complement depleted of $\mathrm{CD}^{+}$cells as detailed in methods. $\mathrm{CD}^{+}$cells from footpad infected mice were then incubated with monoclonal antibodies specific for either V $\beta 6, \mathrm{~V} \beta 7$, or V $\beta 8.1,2$, and 3. Incubated cells were then separated by indirect panning into enriched and depleted fractions of the $\mathrm{V} \beta$ subpopulation 
of interest prior to analysis of anti-viral cytotoxicity by ${ }^{51} \mathrm{Cr}$ release assay, as detailed in methods (A and B are depleted and enriched fractions, respectively). CD8 ${ }^{+}$splenocytes of perorally infected mice were similarly sorted and assessed for anti-viral cytotoxicity (C).156

Figure 10. Intracellular interferon- $\gamma$ staining of a $\mathrm{CD} 8^{+}$splenocytes from perorally infected $\mathrm{C} 3 \mathrm{H}$ mice after 3 weeks of reovirus restimulation in vitro. $5.0 \times 10^{6}$ cell fractions were incubated for 6 hours in the presence of $2.5 \times 10^{6}$ PECs either pulse or unpulsed with reovirus as described in methods. Brefeldin A was added for the last 5 hours of culture. Cells were stained first with anti-Fc $\gamma R$, then with antibodies to $\mathrm{CD} 8 \beta$ and select $\mathrm{V} \beta$ subpopulations. $\mathrm{CD} 8 \beta^{+} \mathrm{V} \beta 6^{+}, \mathrm{V} \beta 8.1,2^{+}, \mathrm{V} \beta 8.3^{+}$, or $\mathrm{V} \beta 12^{+}$and $\mathrm{V} \beta 17^{+}$cells were permeabilized and assessed for the intracellular accumulation of interferon- $\gamma$ with PE-anti-IFN- $\gamma$ antibody.. 158

Figure 11. V $\beta$ repertoire of $\mathrm{CD} 8^{+} \mathrm{T}$ cells recovered from reovirus-infected SCID recipients of immune splenocytes from donor mice infected perorally (A.) or in the hind footpads (B.) one month before. Splenocytes were isolated from recipient reovirus infected SCID mice at days 8,10 , or 12 post-transfer. Data are given as (mean \pm standard deviation) $\times 10^{-6}$ 160

Figure 12. . Immediate ex vivo ${ }^{51} \mathrm{Cr}$ release assay of splenocytes recovered from reovirus infected C3H SCID recipients ten days after adoptive transfer of purified splenic $\mathrm{T}$ cells from donor $\mathrm{C} 3 \mathrm{H}$ mice previously infected perorally (A) or in the hind footpads (B) with reovirus. Recovered splenocytes were complement depleted of either $\mathrm{CD} 4^{+}$cells or $\mathrm{CD} 8^{+}$ cells as described in methods. Control fractions were treated with complement alone. .... 162

Figure 13. $\mathrm{V} \beta$ repertoire of $\mathrm{CD} 8^{+}$lymphocytes recovered from the spleens of infected $\mathrm{C} 3 \mathrm{H}$ SCID recipient mice of perorally infected (A) or naïve (B) donor $\mathrm{C} 3 \mathrm{H}$ mice, ten days after adoptive transfer of Peyer's patch cells as described in methods. Data are expressed as (means \pm standard deviations) $\times 10^{-6} \mathrm{CD}^{+}$cells

Figure 14. Ex vivo ${ }^{51} \mathrm{Cr}$ release assay of splenocytes from reovirus-infected C3H SCID mice 10 days after adoptive transfer of purified Peyer's patch $\mathrm{T}$ cells from naïve $\mathrm{C} 3 \mathrm{H}$ donors or immune donors infected perorally with reovirus one month previously. Splenocytes from infected SCID recipients of immune donor T cells mediate virus-specific cytotoxicity immediately ex vivo, whereas splenocytes of infected SCID recipients of naïve T cells do not.

Figure 15. CDR3 $\beta$ length profiles of $\mathrm{V} 6^{+}$and $\mathrm{V} \beta 2^{+} \mathrm{CD} 8^{+} \mathrm{T}$ cells from the popliteal lymph nodes of footpad infected mice (A) and Peyer's patch cells of orally infected mice (B) compared to cells from non-infected controls. Cells from infected and non-infected mice were harvested 10 days after infection, and were depleted of $\mathrm{CD}^{+}$cells. Cellular RNA was isolated and used as template for RT-PCR using V $\beta 2$ or $\mathrm{V} \beta 6$ and $\mathrm{C} \beta$ primers and assessed using an automated sequencer as described in methods. Data are shown as representative $\mathrm{CDR} 3 \beta$ length profiles for $\mathrm{V} \beta 6$ and $\mathrm{V} \beta 2 \mathrm{CD}^{+}$. No differences are seen in the profiles of cells from infected mice compared to those of non-infected mice immediately ex vivo. .. 168

Figure 16. CDR3 $\beta$ length profiles of $\mathrm{V} 6^{+}$and $\mathrm{V} \beta 2^{+} \mathrm{CD} 8^{+} \mathrm{T}$ cells from the spleens of footpad infected mice and orally infected mice, compared to cells from non-infected controls. Cells from infected and non-infected mice were harvested 10 days after infection, and were depleted of $\mathrm{CD}^{+}$cells. Cellular RNA was isolated and used as template for RT-PCR using $\mathrm{V} \beta 2$ or $\mathrm{V} \beta 6$ and $\mathrm{C} \beta$ primers and assessed using an automated sequencer as described in methods. Data are shown as representative CDR3 $\beta$ length profiles for V $\beta 6$ and $\mathrm{V} \beta 2 \mathrm{CD} 8^{+}$. No differences are seen in the profiles of cells from infected mice compared to those of noninfected mice immediately ex vivo. 
Figure 17. CDR3 $\beta$ length profiles of $\mathrm{V} \beta 6^{+}$and $\mathrm{V} \beta 2^{+} \mathrm{CD} 8^{+} \mathrm{T}$ cells recovered from the spleens of reovirus infected SCID recipients of purified splenic T cells from footpad infected mice or purified T cells from the spleens or Peyer's patches of orally infected donor mice. Cells were recovered from recipient mice 10 days post-transfer, and were depleted of $\mathrm{CD} 4^{+}$cells. Cellular RNA was isolated and used as template for RT-PCR using V $\beta 2$ or V $\beta 6$ and C $\beta$ primers and assessed using an automated sequencer as described in methods. Data are shown as representative CDR3 $\beta$ length profiles for $\mathrm{V} \beta 6$ and $\mathrm{V} \beta 2 \mathrm{CD} 8^{+}$cells. A consistent expansion of $\mathrm{V} 6^{+} \mathrm{CD} 8^{+}$cells using a TCR $\beta$ chain $\mathrm{CDR} 3 \beta$ length is indicated by the arrow

$(\boldsymbol{\Delta})$. No consistent or clear changes are seen in the control V $\beta 2$ profiles. 172

Figure 18. CDR3 $\beta$ length profiles of $\mathrm{V} \beta 6^{+}$and $\mathrm{V} \beta 2^{+} \mathrm{CD} 8^{+} \mathrm{T}$ cells from cultured cell lines established from the spleens of footpad infected mice or orally infected mice. Cells were restimulated in vitro with reovirus for 2 weeks, and were depleted of $\mathrm{CD} 4{ }^{+}$cells prior to analysis. Cellular RNA was isolated and used as template for RT-PCR using V $\beta 2$ or V $\beta 6$ and $C \beta$ primers and assessed using an automated sequencer as described in methods. Data are shown as representative CDR $3 \beta$ length profiles for $\mathrm{V} \beta 6$ and $\mathrm{V} \beta 2 \mathrm{CD} 8^{+}$. A consistent expansion of $\mathrm{V} 6^{+} \mathrm{CD} 8^{+}$cells using a TCR $\beta$ chain $\mathrm{CDR} 3 \beta$ length is indicated by the arrow $(\boldsymbol{\Delta})$. No consistent or clear changes are seen in the control V $\beta 2$ profiles.

Figure 19. CDR3 $\beta$ length profiles of $\mathrm{V} \beta 6^{+} \mathrm{CD} 8^{+} \mathrm{T}$ cells from cell lines established from recovered splenocytes of reovirus infected SCID recipients of purified splenic $\mathrm{T}$ cells from footpad infected mice or purified $\mathrm{T}$ cells from the spleens of orally infected donor mice. Cells were recovered from recipient mice 10 days post-transfer, and were depleted of CD4 ${ }^{+}$ cells. The cells were re-stimulated with reovirus in vitro for a period of 3 weeks. Cellular RNA was then isolated and used as template for RT-PCR using V $\beta 6$ and C $\beta$ primers and assessed using an automated sequencer as described in methods. Data are shown as CDR3 $\beta$ length profiles for $\mathrm{V} \beta 6 \mathrm{CD} 8^{+}$cells. A dramatic and overwhelming expansion of $\mathrm{V} \beta 6^{+} \mathrm{CD} 8^{+}$ cells using a TCR $\beta$ chain CDR3 $\beta$ length is indicated by the arrow ( $\boldsymbol{\Delta})$.V $\beta 2$ profiles were not assessed, as the cell lines from recipients of footpad infected donors and orally infected donors were shown to be $100 \%$ and $65 \% \mathrm{~V} \beta 6^{+}$, respectively....................................... 176

Figure 20. Reovirus-specific and total IgA in LP fragment cultures. Old and young Balb/c mice were infected orally with reovirus, and 10 days later small intestines were removed, fragmented, and cultured for 5 days as described in methods. Reovirus-specific and total IgA concentrations were determined for individual mice by ELISA. Data are expressed in $\mathrm{ng} / \mathrm{ml}$ for reovirus-specific $\operatorname{IgA}$, and $\mu \mathrm{g} / \mathrm{ml}$ for total IgA, as determined by titration of supernatants in comparison to a known murine IgA standards. Reovirus-specific IgA concentration of LP culture supernatants from aged mice was found to be significantly elevated compared to LP culture supernatants from young mice $(p=0.001)$. No significant difference was found in total IgA concentration between old and young mice $(p=0.452) .207$ Figure 21. Splenic reovirus-specific pCTL frequencies of young and old mice. Balb/c (A) and $\mathrm{B} 6 \mathrm{C} 3 \mathrm{~F} 1 \mathrm{C}$ ) mice were infected orally with reovirus and 10 days later spleens were assessed for the frequency of pCTL by limiting dilution analysis as described in methods. Data are expressed as means \pm standard deviation $/ 10^{6} \mathrm{CD}^{+}$cells.

Figure 22. ${ }^{51} \mathrm{Cr}$ release assay of pooled PP lymphocytes from orally infected young and old B6C3F1 mice re-stimulated in vitro with reovirus for 2 weeks. Data are expressed as the mean cytotoxicity against reovirus-infected ( $\boldsymbol{\Delta}$ young PP, $\bullet$ old PP) and non-infected $(\triangle$ young PP, $\bigcirc$ old PP) targets. 
Figure 23. Splenic reovirus-specific IgG AFC frequencies of young and old mice. B6C3F1 (A) and $\mathrm{Balb} / \mathrm{c}(\mathrm{B})$ mice were infected subcutaneously with reovirus and 10 days later spleens were assessed for the frequency of reovirus-specific IgG AFC by ELISPOT analysis as described in methods. Data are expressed as means \pm standard error IgG AFC / $10^{2} \mathrm{Ig} \mathrm{AFC}$.

Figure 24. Reovirus-specific serum IgG titers from young and old mice. B6C3F1 (A) and $\mathrm{Balb} / \mathrm{c}(\mathrm{B})$ mice were infected subcutaneously with reovirus and 10 days later sera were collected and assessed for reovirus-specific IgG by ELISA. Data are expressed as reciprocal mean titer \pm standard error $\operatorname{IgG}\left(\times 10^{-3}\right)$.......

Figure 25. Peyer's patch and splenic CT-specific IgA AFC frequencies of young and old mice.

$\mathrm{B} 6 \mathrm{C} 3 \mathrm{~F} 1$ (A and $\mathrm{C}$ ) and Balb/c (B) mice were immunized orally 3 times with $\mathrm{CT}$ and 7 days following the last immunization PP and spleens were assessed for the frequency of CT specific IgA AFC by ELISPOT analysis as described in methods. Data are expressed as means \pm standard error IgA or IgG AFC / $10^{2} \mathrm{Ig}$ AFC. Young Balb/c mice had significantly more PP CT-specific IgA AFC than old mice $(p=0.015)$; young B6C3F1 mice had significantly more splenic CT-specific IgG AFC than old mice $(p=0.013)$. N.T. $=$ not tested.

Figure 26. CT-specific IgA in fecal pellet extracts (A) and LP fragment cultures (B). Young and old B6C3F1 and Balb/c mice were orally immunized 3 times with $\mathrm{CT}$, and 7 days following the final immunization, small intestines were removed, fragmented, and cultured for 5 days as described in methods. Additionally, fecal pellets formed on day seven following the final immunization were collected and extracted with PBS. CT-specific IgA concentrations were determined for individual mice by ELISA. Data are expressed in ng CT-specific IgA / ml supernatant and ng CT-specific IgA / $\mathrm{ml}$ fecal extract, as determined by titration of samples in comparison to a known murine IgA standard. 219

Figure 27. Splenic reovirus-specific pCTL frequencies of mice infected orally 16 months previously. Mice were either re-infected orally ( $\mathbf{n})$ or not re-infected ( $\mathbf{\square})$, and 10 days later spleens were assessed for the frequency of pCTL by limiting dilution analysis as described in methods. Data are expressed as (means \pm standard deviation) $/\left(10^{6} \mathrm{CD} 8^{+}\right.$cells $)$. Reinfected groups consisted of $n=3$ and $n=2$ mice for experiments 1 and 2, respectively. Not re-infected groups, $n=2$ and $n=3$ mice.

Figure 28. $\mathrm{CD}^{+}$and $\mathrm{CD}^{+}$cell numbers recovered from reovirus infected SCID recipient mice of immune donor T cells. Infected CB.17 SCID mice received $1.5 \times 10^{7}$ purified splenic $\mathrm{T}$ cells from orally infected (A) and hind footpad infected (B) donor mice as described in methods. Splenocytes were isolated from recipient SCID mice at days 9, 11, or 13 posttransfer and assessed for $\mathrm{CD}^{+}(\bullet)$ and $\mathrm{CD}^{+}(\circ) \mathrm{T}$ cells by flow cytometry. Data are given as (mean \pm standard deviation $)\left(\times 10^{-6}\right.$ cells $)$.

Figure 29. V $\beta$ repertoire, in absolute numbers, of $\mathrm{CD} 8+\mathrm{T}$ cells recovered from reovirus-infected SCID recipients of purified $T$ cells from immune donor mice. Infected CB.17 SCID mice received $1.5 \times 107$ purified splenic $T$ cells from orally infected (A) and hind footpad infected (B) donor mice as described in methods. Splenocytes were isolated from recipient SCID mice at days 9,11 , or 13 post-transfer and assessed for changes in the $\mathrm{V} \beta$ repertoire $\mathrm{CD} 8^{+} \mathrm{T}$ cells by flow cytometry, and absolute numbers were calculated. 240 


\section{General Introduction}

Parenteral and enteric viral infections elicit $\mathrm{T}$ cell-mediated immunity tasked with controlling and, insofar as possible, eradicating the invading virus. The tissue environments at distinct anatomic sites of infection may be quite different, and as regards the small intestine Vis a Vis the non-mucosal peripheral immune system, specialized lymphoid tissues and lymphocyte populations exist to tailor the immune response to the unique characteristics of the host tissue. It remains unknown whether distinct anatomic sites of viral infection preferentially select for different $\mathrm{T}$ cell populations to respond to the virus in the setting of the infected tissue.

This introduction serves to review elements of intestinal and non-intestinal systemic immunity, including the priming and effector sites of adaptive immunity, and the immune cells and factors involved in mucosal and systemic immunity to virus infection. Additionally, the model intestinal pathogen, reovirus, is described, in terms of its structure, mechanisms of infection, and the host immune responses it elicits. Finally, consideration of the unique characteristics of the intestinal environment, resident antigen presenting cell populations and responder T cell populations is given with the aim of introducing the hypothesis that an intestinal virus infection may induce the selection and expansion of responder $\mathrm{CD} 8^{+} \mathrm{T}$ cells populations that are distinct from those induced following a parenteral reovirus infection.

A reader may peruse the first part of the introduction to become familiar with the intestinal immune environment, and skip the reovirus section with the understanding that reovirus infection elicits a cytotoxic T lymphocyte (CTL), and that CTL can function to assist in the resolution of reovirus infection. A separate chapter reviewing all the elements of intestinal immunity, especially in humans, is included at the end of this dissertation for the interested reader. 


\section{Intestinal Immunity}

\section{Gastrointestinal immunity: passive or active, innate or adaptive.}

The gastrointestinal system is essentially a long, muscular tube, the functional surface of which is a thin, mucous-coated layer $1 \mathrm{~mm}$ thick that is joined at both ends with the external integument and thus is a contact surface of the external environment (MacDonald et al., 1999). As such, it is in contact with a vast array of environmental antigens ranging from dietary sources and innocuous commensal organisms to frank pathogens. Normal functions of the GI system provide non-specific defenses against foreign organisms (Mowat and Viney, 1997). These defenses include iron-sequestering lactoferrin in saliva and other GI exocrine secretions; peptidoglycan hydrolyzing salivary and gastric lysozyme, secreted complement components such as factor $\mathrm{C} 3$ and factor $\mathrm{B}$, the initiating components of the "alternative pathway" of complement activation; anion-binding pore-forming molecules such as intestinal crypt Paneth cell derived $\alpha$ defensins, and intestinal epithelial cell derived $\beta$-defensins; and Paneth cell secretory phospholipase $\mathrm{A}_{2}$. New defenses are continuously being uncovered, such as the up-regulated production of nitric oxide by duodenal epithelial cells to combat infestation by the parasite Giardia lamblia (Eckmann et al., 2000). Other GI functions with defensive benefits include normal peristalsis, both triggered by bulk in the lumen and normal vagal major motility complexes, gastric parietal cell acid secretion, gastric chief cell pepsin production; pancreatic trypsin, chymotrypsin, phospholipase and bile acids; local insult repair molecules such as intestinal goblet cell derived trefoil proteins (Podolsky, 2000); constant epithelial cell turnover (Merrit et al., 1995); and perhaps most important, the ever present mass of viscous barrier mucous. 
Lymphoid tissues of the intestinal immune system can be divided into two types (Mowat and Viney, 1997). First is the organized priming or inductive tissue such as the Peyer's patches (PP) and mesenteric lymph nodes where antigen-naïve lymphocytes are first activated and induced to proliferate. Second, there are the dispersed effector sites, which include both the lamina propria (LP) underlying the absorptive epithelium, where antigen experienced cells mediate their specialized effector functions such as cytokine secretion and cytotoxicity or antibody production, and the T lymphocyte compartment interspersed within the absorptive epithelial cell layer, containing the so-called intraepithelial lymphocytes (IEL) (MacDonald et al., 1999).

Proceeding distally along the GI tract from the oropharynx, the hostile environment of the stomach gives way to the major absorptive organ of digestion, the small intestine. This is a site of great antigenic load and variability, requiring careful regulation of adaptive immune function. Dangerous organisms must be identified and adequate defenses produced, and yet innocuous dietary antigens must be ignored. This regulation of response vs. non-response is an intrinsic function of the small intestine not normally emulated by most other immune tissues. Additionally, the intestinal mucosal contains newly identified lymphoid-tissue structures, such as the small intestine lymphocyte filled villi (LFV) (Moghaddami et al., 1998) and deep LP crytopatches (Ishikawa et al., 1999), which are of uncertain function, although there is mounting evidence that these tissues may be involved in the generation of unconventional, extra-thymic $\mathrm{T}$ cells which constitute part of the intestinal IEL compartment (Saito et al., 1998; Poussier and Julius, 1999; Oida et al., 2000). 


\section{Sleuth of the sluice; sentinel tissues and immune priming sites that are the Peyer's patches.}

The PP, unlike the peripheral lymph nodes, have no afferent lymphatics, and so the influx of mature, activated dendritic cells that is the central requirement of priming of naïve $\mathrm{T}$ cells does not occur. The PP, in its role as the premier and initial priming tissue of the small intestine has developed specialized structures and tissue organization adapted to its location (Kelsall et al., 1996). The sequence of an active immune response in the small intestine begins with an ingested foreign antigen or organism, such as reovirus, being endocytosed by the microfold cells ( $\mathrm{M}$ cells) of the so-called follicle associated epithelium at the luminal surface of the patch (Owen, 1994). M cells, unlike the columnar absorptive epithelial cells, lack the covering 'molecular sieve' barriers of the glycoprotein glycocalyx and mucous coat on their lumenal surface, and thus are easily able to interact with particulate antigens and organisms in the gut lumen (Nuetra et al., 1996). M cells are thin, endocytic cells that continuously sample the lumenal contents of the small intestine, and transcytose antigen across the $\mathrm{M}$ cell into a region of the PP immediately underlying the FAE known as the 'sub-epithelial dome' that is highly enriched for endocytic dendritic cells (Kelsall et al., 1996). Despite the fact that the M cell is in intimate contact with a population of $\mathrm{T}$ and $\mathrm{B}$ lymphocytes actually encased within pockets of the $\mathrm{M}$ cell membrane (Farstad et al., 1994), antigen processing is not thought to be an important function of the $\mathrm{M}$ cell, and as the M cells have little MHC class II and no co-stimulatory molecule expression, they are thought to not be directly involved in antigen presentation (Farstad et al., 1994). Rather, M cells transport antigen from the intestinal lumen directly into the subepithelial dome region of the PP (Mowat and Viney, 1997). Underlying DCs of the subepithelial dome internalize transcytosed antigen, processing it and presenting it in association with surface MHC molecules during maturation (Keren, 1992). Mature DC migrate from the subepithelial dome region into the T cell 
enriched interfollicular regions of the PP, analogous to the migration of dendritic cells of the non-intestinal periphery to draining lymph nodes. Once in the interfollicular region, mature DC can activate naïve $\mathrm{CD} 4^{+}$and $\mathrm{CD} 8^{+} \mathrm{T}$ cells. Activated T cells are induced first to proliferate, and within one or two cell divisions T cells may take on effector function (Ben-Sasson et al., 2001). Primed T cells may then migrate to the mesenteric lymph nodes via the efferent lymphatic vessels and thence proceed via the thoracic duct directly to the systemic venous circulation (Rothkotter et al., 1999). Like T cells primed in the peripheral lymph nodes, many PP primed cells will deposit in the spleen and continue to proliferate (Bradley et al., 1999). Intestinally primed T cells may leave the PP, MLN, or spleen and circulate via the blood to the LP of the intestine, and to a lesser degree to other mucosal tissues via interactions of post-capillary venule addresin molecules such as MadCAM-1 expressed on the surface of normal or inflamed flat endothelial cells with specific lymphocyte surface integrins such as $\alpha_{4} \beta_{7}$ (Farstad et al., 1996; Wagner et al., 1996). T and B cells exiting the venules of the submucosa take up residence in the LP to mediate effector function, or to await subsequent activation (Molberg et al., 1998). Some of the $\mathrm{T}$ cells, primarily of the $\mathrm{CD} 8^{+}$phenotype and expressing the $\alpha_{\mathrm{E}} \beta_{7}$ integrin, enter the intraepithelial cell compartment, adhering to the palisade-like intestinal epithelial cells (IEC) of the villi via interaction of IEL integrin $\alpha_{E} \beta_{7}$ to IEC E-cadherin (Cepek et al., 1994). Within the $\mathrm{LP}, \mathrm{CD} 4^{+}$cells mediate effector function by producing cytokines to activate resident tissue macrophages and parenchymal cells (MacDonald et al., 1999), and to support antigen-specific B cell differentiation into IgA secreting plasma cells. $\mathrm{CD} 8^{+}$cells within the LP and IEL compartments may mediate their effector function by the killing of infected host cells (Kim et $a l ., 1999)$ and the production of cytokines such as TNF- $\alpha$ and IFN- $\gamma$. 


\section{Intestinal $T$ cells are often of uncertain origin and function $V$ is a $V$ is peripheral $T$ cells.}

The earliest differentiated precursors of T lymphocytes are found in the cortex of the thymus. During a progressive migration of these cells through the medulla they must sequentially successfully rearrange both the $\alpha$ and $\beta$ chains of the TCR. They must then undergo positive selection for the ability to bind self MHC class I or class II molecules, and negative selection to delete $\mathrm{T}$ cells that may become aberrantly activated by self MHC and antigen complexes (Laufer et al., 1999). Although the majority of GALT CD8 ${ }^{+}$cells are thymus-derived (Lin et al., 1996), $\mathrm{CD}^{+} \mathrm{T}$ cells of the GALT are a diverse group, both with regard to function (Mayrhofer, 1980; Guy-Grand et al., 1996), derivation (Mayrhofer, 1980), and cell surface phenotype (Klein, 1986). Antigen-specific $\mathrm{CD}^{+} \mathrm{T}$ cells primed in the PP are thymus-derived, $\mathrm{CD} 8 \alpha \beta$ heterodimer $^{+}$ $\mathrm{TCR} \alpha \beta^{+}$cells, whereas within the intestinal epithelium a minor population of CD8 $\alpha \alpha$ homodimer ${ }^{+} \mathrm{TCR}_{\alpha} \beta^{+}$cells exists which may be extra-thymically derived (Guy-Grand et al., 1991), possibly from the cryptopatches which have been identified in mice, rats, and humans (Saito et al., 1998; Ishikawa et al., 1999), or the lymphocyte filled villi (LFV), which are also conserved among humans and rodents (Moghaddami et al., 1998). There also exists in the epithelium an enrichment of TCR $\gamma \delta^{+}$cells that may express CD8 $\alpha \alpha$ or be $\mathrm{CD}^{-}$, which may be extra-thymically derived (Guy-Grand and Vassalli, 1993). It remains uncertain whether any intestinal CD8 $\alpha \beta^{+}$TCR $\alpha \beta^{+}$cells develop in situ, as opposed to being recruited to the epithelium from the vasculature (Muller et al., 2000). It also remains uncertain as to what immune role extra-thymically derived $\mathrm{T}$ cells of any phenotype actually mediate. 


\section{T cells of the intestinal mucosa are strikingly oligoclonal, by comparison with those of the systemic periphery.}

It has been shown that the TCR $\alpha \beta^{+}$IEL are not polyclonal as are T cells of the peripheral blood and lymphoid tissues (Akolkar et al., 1995), but rather are oligoclonal, and that nearly the total population of TCR $\alpha \beta^{+}$IEL of any individual human or mouse are due to a limited number of highly expanded clones that are distributed along the length of the intestine (Cumano et al., 1994). This suggests proliferation and recirculation of selected populations of TCR $\alpha \beta \mathrm{T}$ cells through the systemic circulation and recruitment back to the mucosal (Arstila et al., 2000). For example, normal human jejunal IEL from six individuals were shown to have expanded representation of one to three TCR V $\beta$ subpopulations accounting for more than $40 \%$ of the total TCR $\alpha \beta^{+}$IEL. Sequencing of cloned PCR products of TCR $\beta$ chain transcripts of one of the overrepresented V $\beta$ subpopulations from two of the donors yielded identical $\beta$ chain sequences in 13 out of 21 clones in one donor. The second donor had a conserved $\beta$ chain sequence in 18 of 21 clones. In both humans and mice, the expanded oligoclonal populations are unique to the individual host, and are distinct from the IEL of even genetically identical animals reared under similar conditions (Cumano et al., 1994). Evidence suggests, however, that despite the oligoclonality of the $\mathrm{CD} 8 \alpha \beta^{+} \mathrm{TCR} \alpha \beta^{+} \mathrm{IEL}$, these cells have immigrated from the peripheral secondary lymphoid tissues. As an example, CD $8 \alpha \beta^{+}$TCR $\alpha \beta^{+}$IEL of DBA/2 mice expressing the minor lymphocyte stimulating antigen alpha $\left(\mathrm{Mls}^{1 \mathrm{a}}\right)$ phenotype were devoid of $\mathrm{V} \beta 6, \mathrm{~V} \beta 8.1$ and V $311^{+} \mathrm{TCR}$ as were $\mathrm{CD} 8^{+} \mathrm{T}$ cells of the peripheral lymphoid tissues (Rocha et al., 1994), whereas $\mathrm{T}$ cells of the deleted phenotype should have been represented in the intestinal mucosal if the cells were derived in situ, thus bypassing normal thymic selection. Nevertheless, these $\mathrm{CD} 8 \alpha \beta^{+} \mathrm{TCR} \alpha \beta^{+}$IEL have been shown to be oligoclonal (Regnault et al., 1994), reflecting V $\beta$ repertoire skewing unseen in the peripheral $\mathrm{CD} 8^{+} \mathrm{T}$ cell compartment, and thus demonstrating 
selective processes on otherwise conventional pCTL not seen outside the intestine. Oligoclonal expansion has also been demonstrated for TCR $\alpha \beta^{+} \mathrm{T}$ cells of the LP, although the T cell population as a whole is far more polyclonal than the IEL. Identical T cell clones have been isolated from the peripheral blood and LP compartments (Kim et al., 1999), from the IEL and LP compartments (Bennet et al., 1999; Kim et al., 1999), and from the thoracic duct, IEL and LP, indicating migration of $\mathrm{T}$ cells to and/or from the peripheral blood, and a common origin of some LP and IEL T cells (Arstila et al., 2000). Additionally, oligoclonality is found within all subsets of LP and IEL TCR $\alpha \beta^{+}$T cells, including CD8 $\alpha \beta^{+}, \mathrm{CD} 8 \alpha \alpha^{+}$(Cumano et al., 1994), and CD4 ${ }^{+} \mathrm{T}$ cells (Arstila et al., 2000), although the same $\beta$ chain sequences are not shared among the $\mathrm{T}$ cells of different subsets.

\section{Antigen-specific cytotoxicity is mediated by intestinal IEL of conventional CD8 $\alpha \beta^{+}$ TCR $\alpha \beta^{+}$phenotype.}

It should be noted that, as with the T cells of the LP, not all the of the TCR $\alpha \beta^{+} \mathrm{T}$ cells of the IEL compartment are members of the expanded oligoclonal populations, and these T cells may be recently arrived CD $8 \alpha \beta^{+}$TCR $\alpha \beta^{+}$T cells primed during incipient immune responses within the PP and other inductive tissues. For example, following infection of mice with reovirus (London et al., 1989; Cuff et al., 1993; Chen et al., 1997), rotavirus (Offit and Dudzik, 1989), LCMV (Sydora et al., 1996; Muller et al., 2000), and T. gondii tachyzoites (Chardes et al., 1994) organism-specific CTL can be isolated from the IEL with the conventional CD $8 \alpha \beta^{+} \mathrm{TCR} \alpha \beta^{+}$ phenotype. This is also true for alloantigen-specific CTL from the IEL compartment in alloantigen-primed mice (Parrott et al., 1983). It is believed that these IEL, like the T cells of the underlying LP, are derived from conventional T cells primed within the PP or peripheral organized lymphoid tissue, as their antigen specific CTL activity is dependent on recent antigen exposure. To date, the oligoclonal CD8 $\alpha \beta^{+} \mathrm{TCR} \alpha \beta^{+} \mathrm{T}$ cell populations of the naïve host IEL 
compartment have not been shown to possess a priori the ability to mediate specific responses against novel antigens, although a recent report suggests that some $\mathrm{CD} 8 \alpha \beta^{+} \mathrm{TCR} \alpha \beta^{+}$IEL from naïve mice can mediate very low levels of LCMV specific cytotoxicity by a Fas-Fas ligand mediated mechanism (Corazza et al., 2000). Nevertheless, expanded oligoclonal IEL populations of CD8 $\alpha \beta^{+} \mathrm{TCR} \alpha \beta^{+} \mathrm{T}$ cells specific for known cognate antigens are present, and are likely derived from immigrant $\mathrm{T}$ cells originally primed in the PP and other peripheral secondary lymphoid tissues (Camerini et al., 1998). Their striking oligoclonality within the IEL compartment is probably reflective of chronic re-stimulation in situ by a restricted number of antigens derived from the diet or the normal intestinal commensals (Imaoka et al., 1996; Arstila et al., 2000), or an uncharacterized stimulatory influence of the intestinal epithelium independent of flora and diet (Guy-Grand et al., 1991; Guy-Grand et al., 1996). Indeed, the V $\beta$ repertoire of germ-free rat $\mathrm{CD} 8 \alpha \alpha^{+}$and $\mathrm{CD} 8 \alpha \beta^{+} \mathrm{TCR} \alpha \beta^{+}$IEL becomes skewed upon microbial colonization of the intestine, whereas the $\mathrm{V} \beta$ repertoire of $\mathrm{CD}^{+} \mathrm{T}$ cells in the mesenteric lymph nodes of these animals remains unchanged, indicating a unique stimulatory environment of the intestinal epithelium associated with normal flora (Helgeland et al., 1996).

\section{Functions of intestinal IEL with unconventional $\mathrm{CD8}^{+} \mathrm{TCR}^{+}$phenotypes.}

Antigen-specific cytotoxicity is not consistently or definitively mediated by the minor

IEL populations of non-conventional CD $8 \alpha \alpha^{+} \mathrm{TCR}_{\alpha} \beta^{+} \mathrm{T}$ cells, nor by the $\mathrm{TCR} \gamma \delta^{+} \mathrm{T}$ cells. Furthermore, these cells do not tend to proliferate in response to antigenic or mitogenic stimulation in vitro (Sydora et al., 1996; Lundquist et al., 1996), although evidence exists that IEL utilize a CD2-dependent co-stimulatory mechanism (Van Houten et al., 1993) and an IL-15 dependent signaling pathway (Inagaki-Ohara et al., 1997) to proliferate following stimulation. Nevertheless, they have been shown to mediate non-specific cytotoxicity in redirected CTL 
assays using anti-TCR $\alpha ß a$ anti-CD3, or $\gamma \delta$ mAb-coated target cells (Viney et al., 1990; Guy-Grand et al., 1991; (Lunquist et al., 1996). These IEL have also been shown to express NK cell surface molecules and mediate protective antiviral NK-like cytotoxicity (Carmen et al., 1986), although unlike conventional NK cells, this killing is not inhibited by target cell MHC class I molecule expression (Guy-Grand et al., 1996). Additionally, human jejunal IEL cell lines have been shown to recognize and kill target cells expressing the non-classical $\beta 2$ microglobulin-associated MHC class I-like molecule CD1c, which may be expressed on stressed or damaged intestinal epithelial cells (Griffith et al., 1998). TCR $\alpha \beta^{+} \mathrm{T}$ cells, which account for $20-80 \%$ of small intestinal IEL in mice (Goodman and Lefrancois, 1988; Hohara et al., 1990), depending on the age and strain (Mosley et al., 1994), are thought to play a role in the maintenance of the integrity of the intestinal epithelium by recognizing and removing stressed or damaged epithelial cells (Yoshikai, 1999), and by producing reparative enterocyte mitogens such as keratinocyte growth factor (Finch and Cheng, 1999). In further evidence of an epithelium-maintaining role, TCR $\gamma \delta^{+}$ T cells have been shown to recognize non-classical MHC-like molecules CD1.1 and the thymic leukemia antigen (TL) in mice (Shawar et al., 1994), and CD1d, MICA, and MICB in humans (Griffith et al., 199; Groh et al., 2000), molecules usually expressed only on distressed intestinal epithelium and professional APC (Shawar et al., 1994), suggesting that TCR $\gamma \delta^{+}$IEL respond to distressed IEC . Additionally, TCR $\gamma \delta^{+} \mathrm{T}$ cells have been shown to spontaneously produce IFN$\gamma \delta$ and IL-5, possibly to modulate other local immune cells (Yamamoto et al., 1993). Evidence also exists for a role of IEL TCR $\gamma \delta^{+} \mathrm{T}$ cells in promoting mucosal IgA responses, as $\beta$ chain gene knock out $\left(\beta^{-/}\right)$mice, which have no TCR $\alpha \beta^{+} \mathrm{T}$ cells, have deficient generation of intestinal $\operatorname{sIg} \mathrm{A}^{+} \mathrm{B}$ cell responses to oral challenge with cholera toxin $\mathrm{B}$ subunit and tetanus toxoid (Fujihashi et al., 1996). Furthermore, TCR $\gamma \delta^{+} \mathrm{T}$ cells appear regulate intestinal TCR $\alpha \beta^{+} \mathrm{T}$ cell 
responses, as $\beta^{-/ 2}$ mice infected orally with the parasite Emeria vermiformis display enhanced severity of TCR $\alpha \beta^{+} \mathrm{T}$ cell mediated mucosal damage in the small intestine compared to similarly infected wild type controls (Roberts et al., 1996). The enhanced pathology can be abrogated by the adoptive transfer of IEL TCR $\gamma \delta^{+} \mathrm{T}$ cells from naïve wild type mice into infected $\beta^{-/-}$recipient mice. While the function of TCR $\gamma \delta^{+} \mathrm{T}$ cells remains incompletely understood, there is ample reason to predict the TCR $\alpha \beta$ T cells of the intestinal mucosa mediate effects distinct from $\mathrm{TCR} \alpha \beta^{+} \mathrm{T}$ cells of the periphery. First, TCR $\alpha \beta \mathrm{T}$ cells make up less than $3 \%$ of $\mathrm{T}$ cells in the spleen and peripheral lymph nodes, whereas they often account for more than half of the murine IEL T cells (Goodman and Lefrancois, 1988; Hohara et al., 1990), and are also enriched in the human intestinal IEL compartment (Bucy et al., 1989). Additionally, the murine intestinal TCR $\alpha \beta^{+}$IEL utilize the TCR V $\beta 7$ segment, whereas those of the periphery mainly use V $\beta 4,5$ and 6 , which suggest distinct TCR specificities of intestinal TCR $\alpha \beta^{+}$T cells compared to those of the periphery (Haas, 1993). The vast majority of the TCR $\alpha \beta^{+}$IEL of the small intestine overlie the LP of the absorptive epithelium, and thus would tend to mediate their effects predominantly outside of the PP. Nevertheless, in view of the evidence that TCR $\alpha \beta^{+}$IEL influence LP lymphocytes, that specialized structures of the LP such as the crytopatches and LFV may serve as an alternate source of non-thymic responder T cells, and the mounting evidence that DC of the LP may prime responder T cells in situ (Muller et al., 2000), the unique influences of the IEL compartment, for which there may be no systemic equivalent, may well be imprinted upon the character of conventional intestinal $\mathrm{T}$ cell responses.

\section{Impact of unique intestinal $\mathrm{CD8}^{+}$responder populations primed in situ.}

It has been suggested that some $\mathrm{CD} 8 \alpha \beta^{+} \mathrm{TCR} \alpha \beta^{+} \mathrm{T}$ cells of the GALT may differ from those of the systemic periphery by their site of origin and priming, as in the case of extra- 
thymically derived $\mathrm{T}$ cells primed within the intestinal mucosa. Additionally, conventional, thymus-derived $\mathrm{CD} 8^{+} \mathrm{T}$ cells induced to respond in the PP may differ from those primed in the systemic periphery due to the unique microenvironment of the PP. Antigen processing and presentation by professional antigen presenting cells such as the dendritic cells of the subepithelial dome region is not definitively known to generate the same antigenic epitopes as APC in the peripheral lymph nodes. Indeed, differences may exist in the $\mathrm{T}$ cell priming and mediated by PP DC as compared with splenic DC that have been attributed to the antigen and cytokine milieu in the intestinal tissues (Hooper et al., 1994). Thus the naïve pool of PP $\mathrm{CD} 8^{+} \mathrm{T}$ cells that could be stimulated to respond to a foreign antigen may differ from that of the peripheral lymph nodes. Additionally, some as yet unidentified influences of the GALT microenvironment may impart unique characteristics on the responding $\mathrm{CD} 8^{+} \mathrm{T}$ cells, such as expression of the IEL phenotype marker, the cell surface integrin $\alpha_{\mathrm{E}} \beta_{7}$, which is induced on $\mathrm{CD} 8^{+}$cells primed in the PP by local transforming growth factor- $\beta 1$ (TGF $\beta 1$ ) production (Kilshaw et al., 1991). Clearly, the understanding of intestinal CTL responses involves a number of complicated elements. Reovirus, a proven inductive agent of systemic and intestinal CTL, provides a useful tool for determining the influence of the anatomic site of infection on CTL immune responses.

\section{Reovirus as a model intestinal and systemic pathogen \\ Reovirus, model infectious organism for the elucidation of mucosal and systemic immune responses.}

Reovirus, respiratory enteric orphan virus, is an icosahedral virus lacking a lipid envelope and glycoproteins (Palmer and Martin, 1977). Reovirus has a genome of 10 double-stranded RNA segments encoding 8 structural and three non-structural proteins (Joklik, 1980; Sakar et al., 1985). The genus Orthoreovirus contains three mammalian reovirus serotypes as determined by hemagglutination inhibition assays, each having a prototypical strain isolated from the 
respiratory or intestinal secretions of human children (Tyler and Fields, 1990). These serotypes are reovirus type 1, strain Lang (T1L), type 2 Jones (T2J), and type 3 strain Dearing (T3D). The most extensively studied serotypes, $\mathrm{T} 1$ and $\mathrm{T} 3$, are naturally infective for the $\mathrm{M}$ cells overlying the small intestinal PP, and for replicating, immature, small intestinal epithelial cells (IEC) of the small intestinal crypts. The outer capsid proteins, $\sigma 1$ and $\sigma 3$, are resistant to both low $\mathrm{pH}$ and lipid detergents, and protect the virus from the hostile environments of the stomach and duodenum. Following ingestion, reovirus undergoes endoproteolytic cleavage of the outer capsid $\sigma 1$ and removal of the $\sigma 3$ proteins due to the action of pancreatic chymotrypsin (Bodkin et al., 1989; Bass $e t a l$, 1990). This processing is an absolute requirement of the natural enteric infection, allowing the remaining intermediate subviral particle (ISVP) to bind to the $\mathrm{M}$ cell of overlying the Peyer's patch (Amerongen et al., 1994). The ISVP can infect the M cell or be transcytosed without further processing to the sub-epithelial dome region of the PP (Wolf et al., 1983), a region rich in dendritic cells.

\section{Natural reovirus T1L infection causes ileal disease.}

Per-oral infection of mice with reovirus T1L causes enteric disease primarily in the ileal crypts of Lieberkuln surrounding the PP, whose M cells serve as portals of egress from the intestinal lumen (Rubin et al, 1985). Reovirus infects and transiently decreases the number of M cells in the first 72 hours following per-oral application (Bass et al., 1988). Transcytosed virus infects dendritic cells and macrophages of the subepithelial dome, which subsequently prime a $\mathrm{T}$ helper cell and CTL response. Reovirus T1 and T3 have also been shown to bind the apical surface of IECs in vitro (Ambler and Mackay, 1991; Weiner et al, 1988 ), and reovirus T1L has been observed to bind the apical surface of goblet cells interspersed among the IEC of the intestinal villi. Although the related genus of Reoviridae, the rotaviruses, do infect the intestinal 
mucosal via the apical surface of mature enterocytes residing at the ends of the enteric villi (Starkey et al., 1986) this route has not been demonstrated for the reovirus. Although reovirus is able to enter cultured monolayers of Caco-2 intestinal epithelial cells via the 'apical' surface, reovirus has only been observed to infect IEC in small intestinal epithelium fragments in vitro via the basolateral surface (Weiner et al., 1988). Binding of reovirus T1L to epithelial fragments was observed to be 100 -fold greater along the basolateral surface than the apical surface, possibly due to interference of binding by the IEC microvillus brush border, the glycoprotein glycocalyx and the goblet cell-secreted mucous coat overlying the absorptive epithelium. PP M cells have no brush border or glycocalyx coating, and are free of secreted mucous, possibly facilitating virus binding (Neutra et al., 1996), and electron micrographs have shown that reovirus ISVPs have tendency to exclusively bind M cells in vivo (Bass et al., 1988). Thus, access to the epithelium is thought to be most likely due initially to infection or penetration of PP M cells by reovirus, which then extends either directly through the lamina propria to the basolateral surface of the crypt enterocytes or by dissemination of reovirus via the local circulation either freely or in association with infected APC. However, given the huge surface area of the small intestinal epithelium, estimated in humans to be approximately $400 \mathrm{~m}^{2}$ (Mowat and Viney, 1997), may allow for even exceptionally rare direct infection of enterocytes of the absorptive epithelium via the apical surface, thus bypassing the $\mathrm{M}$ cells, to be a significant pathway of infection in vivo. A recent report by Barton et al., (2001) that reovirus T1 and T3 can bind the junction adhesion molecules found in the tight junctions of a number of mammalian tissues, including the polarized epithelial cells of the intestine, lends support to the idea that reovirus may gain access to the absorptive epithelium directly, possibly through minor ruptures 
in the tight junctions between epithelial cells. Nevertheless, the majority of reovirus virions are believed to cross the M cell of the FAE.

Following transcytosis through the $\mathrm{M}$ cell into the sub-epithelial dome of the PP, the ISVP binds to and is internalized by host APC via receptor-mediated endocytosis (Wolf et al., 1981). The $\square 1$ hemagglutinin tetramers in the 12 vertices of the icosahedral ISVP bind sialyated glycoproteins on the surface of host cells (Kauffman et al., 1983). The endosomes containing the endocytosed ISVPs then fuse with lysosomes (Schiff and Fields, 1990). Further chymotryptic degradation by lysosomal proteases is thought to expose lipophilic amino acid residues of the subviral core particle, which facilitate fusion of the core with the lysosomal membrane phospholipids and subsequent escape of the core into the cytoplasm, allowing for genome transcription and viral replication (Schiff and Fields, 1990).

Reovirus is a cytopathic, lytic virus that ruptures the cell membrane of its host cell to allow dissemination of progeny virions, in addition to large amounts of non-infectious virus particle debris (Oberhaus et al., 1998). Infectious virions may escape the Peyer's patch through the draining lymphatics or by direct extension into the lamina propria of the small intestinal absorptive epithelium from the follicle associated epithelium, sequentially infecting ileal crypt IEC (Rubin et al, 1985) along their basolateral surfaces (Weiner et al., 1988) by binding to the epithelial growth factor receptor (Strong et al., 1993; Tang et al., 1993). Productive infection of immature crypt enterocytes by reovirus may be due to a requirement for factors available only in replicating host cells to support viral replication.

\section{Reovirus elicits innate immune responses.}

Reovirus type 1 and 3 infections of L929 murine fibroblasts in vitro have both been shown to induce an increase in IFN- $\alpha$ (type 1 interferon) production. The induction of IFN- $\alpha$ is 
due to a cascade of events subsequent to the activation of cellular dsRNA-dependent protein kinase by the dsRNA product of transcription of viral mRNA from negative sense viral template RNA, and is inhibited by binding of particles of the $\sigma 3$ structural protein to viral template RNA (Samuel, 1998). Type 1 interferons have been shown to play a protective role in murine infection with other cytopathic viruses such as vaccinia virus (Nagao et al., 1998), influenza A virus (Durbin et al., 2000), and vesicular stomatitis virus (van den Broek et al., 1995). The role of type 1 interferons in vivo has not been determined for reovirus infection nor for rotavirus infection. While reovirus infected cells are susceptible to NK cell mediated lysis, the role of NK cells in responding to reovirus infection has also not been thoroughly evaluated in vivo (Fawaz et al., 1999), although potent NK responses have been demonstrated during reovirus infections of athymic nude mice and CB.17 SCID mice (Taterka et al., 1995).

\section{Reovirus elicits adaptive immune responses.}

Reovirus-specific adaptive immunity has been extensively studied. Intraperitoneal or intragastric infection of immunocompetent mice with reovirus T1L induced Thy- $1^{+} \mathrm{T}$ cells that could mediate delayed type hypersensitivity (DTH) responses following injection of reovirus into the footpads of immune mice (Weiner et al., 1980; Letvin et al., 1981). Further, Thy-1 ${ }^{+} \mathrm{T}$ cells isolated from the spleens of immune mice could be induced to mediate anti-reovirus CTL effector function following re-stimulation in vitro with reovirus-pulsed APC. The generation of reovirus-specific CTL and DTH was dependent on professional APC, as mice depleted of professional APC by UV irradiation prior to infection had diminished ability to generate reovirus specific T cell immunity (Letvin et al., 1981). 


\section{The phenotype and function of reovirus-specific CTL.}

Reovirus-specific CTL have been shown to be H-2 (MHC class I) restricted (Ertl et al., 1982). Reovirus-specific CTL could be elicited in mice to viral epitopes in association a broad range of murine class I alleles (Parker and Sears, 1990). APC within the PP, such as dendritic cells or macrophages, prime the reovirus-specific CTL response following enteric reovirus infection (Letvin et al., 1981). It is unknown if dendritic cells or macrophages containing reovirus or even free virus particles gaining access to the mesenteric lymph node via the PP efferent lymphatics or lacteals draining the intestinal villi prime CTL in tissues other than the PP. However, replicating reovirus has been recovered from both the MLN and the spleen following enteric infection, suggesting that CTL could be primed outside the PP during enteric infection (Letvin et al., 1981). Reovirus-specific CTL can be derived from cultures of MLN, spleens, peripheral lymph nodes, and IEL in addition to PP following enteric infection, though it is uncertain whether these CTL were primed in situ or migrated to these lymphoid compartments after priming in the PP. Murine enteric reovirus T 1L infection resulted in precursor CTL (pCTL) frequencies by day 6 post-infection up to 100 times higher in the PP than the MLN, spleen, or PLN, as determined by LDA (London et al, 1987). A similar observation of graded pCTL frequencies was made in rat lymphoid tissues following enteric rhesus rotavirus infection. Nevertheless, the question of where the pCTL outside the PP were initially primed is still valid (Offit et al., 1991). It seems likely, however, that the majority of pCTL primed following enteric infection comes from the PP, with some extra-intestinal priming in the MLN and spleen.

CTL primed in the PP of mice enterically infected with reovirus T1L have been shown to be conventional class I MHC-restricted CD8 $\alpha \beta^{+} \mathrm{TCR} \alpha \beta^{+}$cells expressing the effector / memory hyaluronate adhesion molecule Pgp-1 (CD44), Thy-1 (London et al., 1987), and the novel 
germinal center T cell associated marker GCT (London et al., 1990). Reovirus-specific CTL have been isolated from the IEL compartment of enterically infected mice (London et al., 1989; Cuff et al., 1991; Chen et al., 1997), and as there exist unconventional CD $8^{+}$cells in the IEL, the origin of these cells and the site of their priming with reovirus are uncertain. Reovirus specific CTL derived from the PP were subsequently shown to be similar in phenotype to the reovirusspecific CTL populating the small intestinal IEL compartment following enteric reovirus infection in mice (Cuff et al., 1991). Additionally, the frequency of virus-specific pCTL among $\mathrm{CD}^{+} \mathrm{PP}$ cells following enteric infection, was similar to that of $\mathrm{CD} 8^{+} \mathrm{IEL}$, approximately 200/10 . Furthermore, adoptively transferred PP CTL populated the IEL compartment of SCID recipient mice, and persisted in situ for up to 4 weeks. Enteric reovirus T1L infection of $\mathrm{C} 3 \mathrm{H}$ mice has been shown to induce reovirus-specific CTL in the IEL compartment predominantly bearing TCR V $\beta 12$ or V $\beta 17$, with minor responder populations expressing V $\beta 2,7,9$, or 14 (Chen et al., 1997). These CTL are possibly conventional thymus-derived CD $8^{+} \mathrm{T}$ cells originally primed by dendritic cells within the PP.

The 3 serotypes of reovirus, T1, T2, and T3, were differentiated by antibody mediated blocking of viral hemagglutination. CTL responses to the three serotypes were initially shown to be completely serotype-restricted to unique epitopes derived from the product of the S1 gene, which encodes the viral cell attachment protein $\sigma 1$ (hemagglutinin) (Finberg et al., 1979). Reovirus-specific CTL induced by parenteral infection were later demonstrated to be serotype non-restricted, or cross-reactive among all three serotypes, presumably by recognizing common epitopes in association with a wide spectrum of murine H-2 molecules (Parker and Sears, 1990). CTL recovered from the PP and intestinal epithelium following enteric infection were similarly shown to be cross-reactive against target cells infected with reovirus T1L or T3D (London et al., 
1989b). Subsequently, clones of reovirus-specific CTL induced following per-oral infection were shown to be predominantly cross-reactive among the three serotypes, with very rare clones specific for T1 or T3 alone (Hogan and Cashdollar, 1991). Most recently, a second protein product from the bi-cistronic S1 gene (Munemitsu et al., 1986), the $\sigma 1$ non-structural ( $\sigma 1 \mathrm{NS}$ ) protein (Sarkar et al., 1985), was identified as containing a cross reactive epitope for CTL generated from $\mathrm{C} 3 \mathrm{H}$ mice infected with reovirus types 1 and 3 (Hoffman and Cashdollar, 1996). It is as yet unknown if all CTL epitopes are derived from the $\sigma 1 \mathrm{NS}$ protein.

\section{$\mathrm{CD8}^{+} \mathrm{CTL}$ play a role in clearing reovirus infection, but are not necessarily a sine qua non of effective anti-reovirus immunity.}

The role of $\mathrm{CD} 8^{+} \mathrm{CTL}$ in the response to reovirus infection has been extensively studied. It has been established that $\mathrm{CD} 8^{+}$cells have an important role in the antiviral immune response, although they are not absolutely required to clear reovirus infection. $\beta 2 \mathrm{~m}$ gene knock out $(\beta 2 \mathrm{~m}$ $\mathrm{KO}$ ) mice lacking conventional $\mathrm{CD} 8^{+} \mathrm{T}$ cells clear reovirus $\mathrm{T} 1 \mathrm{~L}$ infection within 10 to 14 days, kinetics that are comparable to wild type controls (Major and Cuff, 1997). $\beta 2 \mathrm{~m} \mathrm{KO}$ mice were similarly able to clear reovirus $\mathrm{T} 3$ infection within 7 days, the same time required for wild type controls (Barkon et al., 1996). Nude mice lacking $\mathrm{CD} 4^{+}$and $\mathrm{CD} 8^{+} \mathrm{T}$ cells are thought to be capable of controlling reovirus $\mathrm{T} 1$ infection following intraperitoneal injection, though at the day nine post-infection time point at which intestinal and lymphoid tissues were assessed for replicating reovirus it was merely demonstrated that the nude mice had comparable amounts of virus as euthymic controls, but had not yet cleared the virus (Letvin et al., 1981). Furthermore, euthymic mice depleted of APC by UV irradiation, and thus defective in the generation of virusspecific CTL and Thelper cell responses were able to clear reovirus infection as rapidly as nonirradiated mice (Letvin et al., 1981). Thus, $\mathrm{CD} 8^{+}$cells, and possibly $\mathrm{CD} 4^{+}$cells are not required for normal clearance of reovirus infection of mice. B cells are apparently also not absolutely 
required, as B cell deficient MuMT mice, which have a null mutation of the IgM transmembrane exon, clear reovirus infection with only a slight delay relative to wild type controls (Barkon et al., 1996). Nevertheless, SCID CB17 mice which have neither functional T nor B cells, and which mount a furious NK cell response to enteric reovirus T1L infection (Taterka et al., 1995), are unable to clear reovirus and die within six weeks post-infection of hepatic necrosis (George et al., 1990). Thus, while either cellular or humoral immunity are dispensable in clearance of reovirus infection, they are not both simultaneously dispensable. These observations are similar to those reported for rotaviruses (Franco and Greenberg, 1995; McNeal et al., 1997).

\section{Reovirus-specific lymphocytes protect against reovirus-induced pathology.}

The roles of T cells and B cells in the reovirus-specific immune response have been further assessed by the transfer of fractionated lymphocyte populations into reovirus-infected immunodeficient mice. As mentioned, SCID mice enterically infected with reovirus T1L or T3D cannot clear reovirus infection and die within 6 weeks (George et al., 1990). While replicating virus can be isolated from numerous organs, including the small intestinal crypts, the spleen, and the Purkinje cells, massive viral replication and necrosis occurs among liver hepatocytes. Transfer of $1.5 \times 10^{7}$ unfractionated PP cells from donor mice previously infected intraduodenally with the homologous serotype of reovirus into SCID recipient mice 2 days before challenge with reovirus could contain the infection within the small intestines of the SCID mice and completely clear the virus within the second week post-infection. Transferred PP cells from non-immune mice could also clear replicating virus within 2 weeks post-infection, although the reovirus infection could not be contained initially within the small intestine as it was by the immune PP cells. The protective effect of transferred PP cells was titratable, as $5 \times 10^{6}$ transferred immune cells mediated somewhat delayed protection, whereas $1 \times 10^{6}$ cells were not protective. 


\section{Reovirus-specific CTL help protect against visceral organ disease.}

It was subsequently observed that infection of visceral organs outside the intestines, such as the heart, was attenuated by transferred immune splenocytes to neonatal mice prior to infection (Virgin and Tyler, 1991). Neonatal mice transferred with immune splenocytes 2 days prior to infection with myocarditis -inducing reovirus strain T3/8B were protected from virusinduced myocarditis, whereas recipients of cells from naïve donors were not protected from myocarditis, although both immune and naïve cells eventually completely cleared replicating reovirus (Sherry et al., 1993). Together, these results indicate that reovirus can cause severe pathology in immunodeficient mice, which can be contained by the lymphocytes of adaptive immunity.

\section{Conventional and unconventional CTL are induced in respiratory tract associated lymphoid tissue following infection of the respiratory tract with reovirus.}

Reovirus-specific CTL are induced during murine bronchopneumonia following intratracheal instillation of reovirus T1L (Thompson et al., 1996; Periwal and Cebra, 1999). $\mathrm{CD} 8^{+}$reovirus-specific $\mathrm{pCTL}$ can be recovered from the draining mediastinal and tracheobronchiolar lymph nodes, and induced to mediate virus specific cytotoxicity following in vitro re-stimulation. Additionally, a $\mathrm{CD} 8^{+} \mathrm{T}$ cell infiltrate of putative $\mathrm{CTL}$ can be recovered from the airspaces by bronchoalveolar lavage in the first week post-infection (Thompson et al., 1996). The most intriguing result of reovirus T1L bronchopneumonia is the generation of an unusual population of $\mathrm{CD}^{+} \mathrm{CD}^{+}$double positive (DP) $\mathrm{T}$ cells in the draining mediastinal lymph nodes that are also capable of mediating reovirus-specific cytotoxicity following in vitro re-stimulation (Periwal and Cebra, 1999). While peripheral populations of mature DP T cells have been found in rats (Helgeland et al., 1999), mice (Das and Janeway, 1999), humans (Colombati et al., 1998), macaques (Currier et al., 1999), and pigs (Zuckermann and Gaskins, 1996), they usually express 
the non-conventional CD8 $\alpha \alpha$ homodimeric phenotype, whereas DP cells elicited by reovirus bronchopneumonia express the conventional CD8 $\alpha \alpha$ heterodimer, and are thus of uncertain lineage. Together, these experiments indicate an important role for $\mathrm{CD} 8^{+} \mathrm{T}$ cell in the immune response to reovirus infection at a number of anatomic locations. Furthermore, as demonstrated by Periwal and Cebra (1999) following intratracheal instillation of reovirus, the anatomic site of reovirus infection can influence the generation of unique reovirus-specific CTL populations. It remains unknown what differences exist among CTL populations induced following enteric infection with reovirus as opposed to infection at peripheral non-mucosal locations. Research outlined in the following chapters will address this question.

\section{The $T$ cell receptor; rearrangement, selection, and function \\ Rearrangement of the TCR gene segments during $T$ cell ontogeny; specificity through variability.}

$\mathrm{T}$ cells play an important role in adaptive immune responses, which are directed specifically against invading organisms and foreign antigen. Conventional $\mathrm{T}$ cells usually respond specifically to unique peptide sequences presented on the surface of host cells in association with class I or class II major histocompatibility complex (MHC)-encoded proteins. To limit the $\mathrm{T}$ cell response solely to peptide sequences unique to foreign antigens, and conversely to avoid responding to self-peptides, the $\mathrm{T}$ cell relies on the exquisite antigen specificity of its T cell receptor (TCR) (Kaye et al., 1992; Sandberg et al., 2000). The TCR is a heterodimeric molecule of an $\alpha$ and $\beta$ chain (Samelson et al., 1985), the distal ends of which have variable protein structural conformations unique to any given naïve $\mathrm{T}$ cell and its progeny that confer upon the $\mathrm{T}$ cell its antigen specificity(Goyarts et al., 1998; Garcia et al., 1998). The $\alpha$ and $\beta$ chain genes are composites of germ line DNA segments that undergo rearrangement during T cell ontogeny (Kronenberg et al., 1986). The $\beta$ chain gene is a composite of a single V $\beta$ 
gene element, a diversity (D $\beta$ ) element, and a joining $(\mathrm{J} \beta)$ element. Post-transcriptional modification of nascent $\beta$ chain mRNA involves the splicing of the rearranged $V \beta D \beta J \beta$ gene mRNA with the mRNA encoding a non-rearranged constant $(C \beta)$ element, yielding a complete $\beta$ chain mRNA with the order $\operatorname{V} \beta \mathrm{D} \beta \mathrm{J} \beta \mathrm{C} \beta($ Kronenberg et al., 1986). The rearrangement of the $\beta$ chain gene elements and post-transcriptional splicing are similar except that there are no $\alpha$ chain diversity (D) elements (Winoto et al., 1985; Kronenberg et al., 1986; Yague et al., 1988). The $\alpha$ and $\beta$ chains each contain 3 structurally variable regions, called complementarity-determining regions (CDR) responsible for the specific recognition of the cognate antigen in association with a specific self-MHC molecule, known as the restricting MHC molecule (Teng et al., 1998; Garcia et al., 1998). The CDR1 and CDR2 regions of the $\beta$ chain (CDR1 $\beta$ and CDR2 $\beta)$ are encoded completely by the rearranged $\mathrm{V} \beta$ gene element. The CDR3 $\beta$ region is, by contrast, encoded by the site of joining of the rearranged $\mathrm{V} \beta$ gene element and the rearranged $\mathrm{J} \beta$ gene element. Similarly, the CDR $1 \beta$ and CDR $2 \beta$ are encoded by the rearranged $V \beta$ gene element, with the CDR3 $\beta$ region encoded by the joining of the $\operatorname{V\beta D} \beta \mathrm{J} \beta$ elements, inclusive of the entire $\mathrm{D}$ element. The CDR 1 and CDR $2 \beta$ and $\beta$ regions are responsible for recognizing and binding the restricting self-MHC allele, with the CDR3 $\beta$ and to a lesser extent the CDR3 $\beta$ regions responsible for recognizing the cognate antigenic peptide epitope (Garcia et al., 1998). Recently, the CDR1 $1 \beta$ region has been shown to play a minor supporting role in antigen recognition as well (Pannetier et al., 1991). That the CDR3 $\beta$ and CDR1 $\beta$ are derived in part or in whole from the selected $\mathrm{V} \beta$ gene element, and that these regions contribute to the majority of cognate antigen recognition (Pannetier et al., 1991), allows the use of monoclonal antibodies specific for distinct, invariant sequences within the amino acid chain encoded by a given $\mathrm{V} \beta$ element as phenotypic 
markers of $\mathrm{T}$ cell populations utilizing that $\mathrm{V} \beta$ element in the $\beta$ chain of their TCR (Faint et al., 1999).

Random rearrangement of $\beta$ chain elements allow for the characterization of antigendriven proliferative expansion of T cells bearing identical $\beta$ chain sequences by CDR3 $\beta$ length profile analysis.

Rearrangement of $\beta$ chain gene elements to give T cells bearing TCR of unique sequences and specificities has been exploited to allow identification of proliferative expansion by responder $\mathrm{T}$ cells of a known $\mathrm{V} \beta$ phenotype. The unique sequences of the CDR3 $\beta$ region are due to the messy process of rearrangement of the $\mathrm{V} \beta \mathrm{D} \beta \mathrm{J} \beta$ elements. The $\beta$ chain must be derived of elements that rearrange to give a gene that is in-frame for appropriate translation. Thus, in addition to the randomly cut and ligated portions of the stochastically selected $\mathrm{V} \beta, \mathrm{D} \beta$, and $\mathrm{J} \beta$ elements in a given $\mathrm{T}$ cell, random, non-templated bases are added during the rearrangement process that both facilitate ligation and provide an in-frame $\beta$ chain of variable length, always varying by multiples of 3 . The variable amino acid sequence of the CDR3 $\beta$ region grants a $\mathrm{T}$ cell and all of its progeny a unique antigen specificity. Proliferation of a given T cell will create numerous progeny bearing identical TCR, and thus identical CDR3 $\beta$ sequences and lengths. Thus, changes in the distribution of all CDR3 $\beta$ lengths for a responder $\mathrm{V} \beta$ subpopulations following exposure to cognate antigen allows for a more detailed assessment of the antigen-specific $\mathrm{T}$ cells. The 'CDR3 $\beta$ length profile' is the distribution of PCR products of cDNA derived from the mRNA for TCR $\beta$ chains. Primers specific for elements of the TCR flanking the CDR3 $\beta$ region are selected to look at the CDR3 $\beta$ length profiles of the $\mathrm{V} \beta$ populations of interest. The primers allow for the PCR amplification of the variable length CDR3 $\beta$ regions from a population of $\mathrm{T}$ cells, that in the unimmunized state, give a normal distribution of DNA fragments varying from each other by 
multiples of 3 base pairs. Following exposure to cognate antigen, proliferative expansion of responder T cells generate large numbers of progeny $\mathrm{T}$ cells with identical TCR. Thus, the immunized $\mathrm{T}$ cell population will not show a normal distribution of CDR3 $\beta$ PCR fragment lengths, but will instead show a skewing of the distribution to reflect the expansion of $\mathrm{T}$ cells bearing an identical CDR3 $\beta$ length (Pannetier et al., 1993). This technique can be used like flow cytometric $\mathrm{V} \beta$ repertoire analysis to determine antigen-specific $\mathrm{T}$ cell subpopulations on the basis of CDR3 $\beta$ length profile 'skewing' associated with antigen exposure, and provides a further insight into the clonalality of the $\mathrm{T}$ cell response, on the assumption that responder $\mathrm{T}$ cells bearing TCR utilizing the same V $\beta$ element, but having different CDR3 $\beta$ sequences, and possibly lengths, will give distinct patterns of CDR3 $\beta$ length profile skewing.

\section{Germ-line polymorphism governs the potential of the TCR V $\beta$ repertoire.}

As mentioned previously, the TCR $\beta$ chain is responsible for the majority of the antigen recognition. As the $V \beta$ element contains the entire $C D R 1 \beta$ region and contributes to the $C D R 3 \beta$ region that mediates TCR $\beta$ chain antigen recognition, the selected $\mathrm{V} \beta$ element of any given TCR $\beta$ chain determines much of a given T cell's antigen specificity (Pannetier et al., 1993). Thus, the available $\mathrm{V} \beta$ repertoire utilized by peripheral $\mathrm{T}$ cells can influence the generation of an adaptive immune response to a foreign organism or antigen (Cao et al., 1996). Anything that alters the available T cell TCR V $\beta$ repertoire can affect the magnitude or character of the $\mathrm{T}$ cell response. A number of factors can affect the $\mathrm{V} \beta$ repertoire available in the peripheral $\mathrm{T}$ cell pool. Germ-line V $\beta$ gene elements present in one animal strain or individual may be deleted in another. For example, mice of the $\mathrm{V} \beta{ }^{\mathrm{a}}$ haplotype have a deletion of the $\mathrm{V} \beta 5,8,9,11,12$, and 13 elements, all of which are retained in mice of the $\mathrm{V} \beta^{\mathrm{b}}$ haplotype, radically altering the potential antigenic specificities of the $T$ cell pool available to $V \beta^{\mathrm{a}}$ haplotype mice as compared to $V \beta^{\mathrm{b}}$ 
haplotype animals (Osman et al., 1999). Additionally, mutations have led to polymorphisms within the germ-line Vßelements, changing the resultant amino acid sequences, which has been demonstrated to possibly influence thymic selection processes, and thus the peripheral $\mathrm{T}$ cell pool, as well as peripheral immune responses to cognate antigen (Osman et al., 1999). Other mutations may render a $\mathrm{V} \beta$ element nullified, as in the nonsense mutation within the $\mathrm{V} \beta 17$ gene element in C3H mice (Wade et al., 1988).

\section{Intra-thymic post-rearrangement negative selection of the TCR repertoire congenital factors.}

Intra-thymic selection processes following successful rearrangement the TCR $\alpha$ and $\beta$ chains bring new influences to bear on the immature DP T cell. The positive selection of DP T cells has been demonstrated to be dependent mostly on the selected $\mathrm{V} \beta$ element of the $\beta$ chain (Wang et al., 1998), with preferential usage of some V $\beta$ elements among T cells recognizing class I or class II molecules (Manning et al., 1999). There is, however, MHC selective pressure on some $\mathrm{V} \beta$ elements, as in the positive selection of TCR $\mathrm{V} \beta 5^{+}, \mathrm{V} \beta 8.2^{+}, \mathrm{V} \beta 9^{+}, \mathrm{V} \beta 12^{+}$, and

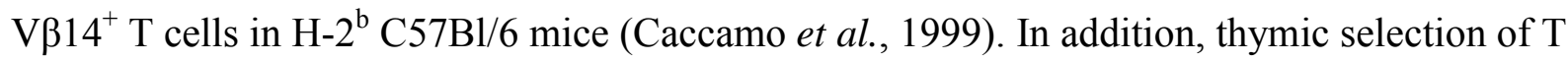
cells with TCR expressing distinct V $\beta$ elements has been strongly associated with provirusencoded endogenous superantigens. Endogenous retroviral superantigens, known as minor lymphocyte stimulating (Mls) antigens for the ability to induce allogenic mixed lymphocyte reactions among MHC haplo-identical mice, are derived from the protein products encoded by the long terminal repeats of murine mammary tumor viruses (MMTV)(Tomonari et al., 1992; Jarvis et al., 1994). There are 4 classic Mls antigens $\left(\mathrm{Mls}^{1-4}\right)$ the expression of which by thymic epithelial cells result in the negative selection of DP T cells expressing certain V $\beta$ elements, a phenomenon known as clonal deletion (Acha-Orbea and MacDonald, 1995). For example, H-2 ${ }^{\mathrm{d}}$ mice expressing Mls ${ }^{1 a}$ have intra-thymic deletion of all DP T cells expressing V $\beta 6$. Thus, no 
$\mathrm{V} 6^{+} \mathrm{CD} 4^{+}$or $\mathrm{CD} 8^{+}$cells are allowed to mature or be exported to the periphery. $\mathrm{DBA} / 2$ mice are devoid of $\mathrm{V} \beta 3,6,7$, and $17^{+}$cells due to intra-thymic expression of an endogenous MMTV superantigen (Marodon and Rocha, 1994). There are up to 30 endogenous retroviruses of the MMTV type integrated into the murine genome, with any given strain expressing 3 to 8 viral proteins to varying degrees (Acha-Orbea and MacDonald, 1995). It is unknown what influence endogenous retroviral proteins may have on human thymic selection processes.

\section{Extra-thymic modification of the TCR repertoire of the mature peripheral T cell pool.}

There are also extra-thymic influences in the periphery that further shape the $\mathrm{T}$ cell repertoire of $\mathrm{T}$ cells surviving the thymic selection processes. Normal antigen specific responses induce the expansion, often quite dramatically, of antigen specific $\mathrm{T}$ cells in the periphery, and this is especially true for virus-specific $\mathrm{CD} 8^{+} \mathrm{T}$ cell populations (Mongkolsapaya et al., 1999). There are also mitogenic influences on the peripheral $\mathrm{T}$ cell repertoire mediated by bacterial (Sundstedt et al., 1998; Haut et al., 1999), parasitic (Denkers et al., 1994), and viral superantigenic proteins (Smith et al., 1993), which can have long-term effects on T cell immunity. For example, bacterial superantigens are thought to play a role in activating and causing the proliferative expansion of $\mathrm{CD} 4^{+}$and $\mathrm{CD} 8^{+} \mathrm{T}$ cells expressing $\mathrm{V} \beta 8$ in the bronchus associated lymphoid tissues of humans with poorly controlled chronic asthma (Haut et al., 1999).

\section{Positive and negative selection of mature peripheral $T$ cells by superantigens.}

Superantigens influence the peripheral $\mathrm{T}$ cell repertoire by interacting with conserved sequences of select TCR V $\beta$ elements and the $\beta 2$ domain of MHC class II molecules, causing T cell activation and proliferation, and often though not always subsequent deletion or anergy of the responder T cells (Sundstedt et al., 1995). Protein homogenates of Toxoplasma gondii tachyzoites have been shown to cause an MHC class II dependent expansion of the $\mathrm{V} \beta 5^{+} \mathrm{CD} 8^{+}$ 
peripheral T cells in C57BL/6 mice (Denkers et al., 1994), an example of the ability of superantigens, which preferentially activate $\mathrm{CD} 4^{+}$cells in association with MHC class II molecules, to also activate $\mathrm{CD} 8^{+} \mathrm{T}$ cells by cross-linking their TCR V $\beta$ element with otherwise non-restricting MHC class II molecules (Sundstedt et al., 1995; Sundstedt et al., 1998). Additionally, viral superantigen(s) of a transmissible B cell tropic MMTV passed in the milk of lactating Balb/J dams to their offspring are expressed on infected PP B cells, causing deletion throughout the peripheral lymphoid tissues of ${\mathrm{V} B 6^{+}}^{\mathrm{T}}$ cells $\mathrm{CD} 4^{+}$and $\mathrm{CD} 8^{+}$(Tucek et al., 1993). Thus, genetic background, intra-thymic selection events, and post-thymic proliferation and deletion events serve to shape the peripheral $\mathrm{T}$ cell repertoire.

It seems certain that in shaping the possible responder $\mathrm{T}$ cell pool, factors such as intrathymic and extra-thymic selection and deletion of the available $\mathrm{T}$ cell pool may influence antigen-specific immune responses. Other influences, such as the microenvironment of the anatomic site of infection, the infecting agent, and the antigen presenting cell population, also shape the immune response. Nowhere is this more evident than the distinct features of immunity following immune priming at a mucosal location as compared to priming at a non-mucosal site.

\section{The CTL response to virus infection}

\section{General characteristics of viral infection and the innate host immune response.}

Virus infections elicit varied immune responses dependent upon the infecting virus, the host tissue, the immune capability of the host, and other factors. Nevertheless, there are stereotypical features of the immune response. A typical virus infects a host tissue, initially adhering to the cell membrane by a specific viral ligand-binding event called adsorption (Perez and Carrasco, 1994). Internalization of the virus occurs by direct membrane fusion in the case of enveloped virus such as influenza A and B (Roth et al., 1986), and Sendai virus (Sechoy et al., 
1989) or by receptor mediated endocytosis for non-enveloped viruses, such as poliovirus or reovirus (Perez and Carrasco, 1994). Depending on the viral products produced in the cytosol, and especially in response to dsRNA intermediaries produced by some viruses such as reovirus, innate cellular defensive measures, such as production of type 1 interferon $\alpha$ and $\beta$, attempt to forestall viral reproduction by shutting down ribosomal translation and inducing enzymatic cleavage of all cytosolic mRNA (Samuel, 1998). Viral hijacking of cellular translational machinery results in decreased cell surface MHC class I molecule expression, rendering infected cells susceptible to killing by natural killer (NK) cells, which are otherwise inhibited by cellsurface expression of normal levels of MHC class I (Huard and Fruh, 2000). NK cells probably play an important role in suppressing the spread of the virus until virus-specific lymphocytes can be activated to clear the infection. NK cell activity is increased by local type 1 interferon, which induces NK cells to produce the anti-viral Type 2 interferon, interferon- $\gamma$ (IFN- $\gamma$ ) (Biron, 1999) which enhances CTL responses, increases MHC class II expression, and recruits circulating lymphocytes to sites of active inflammation (Parr and Parr, 2000). Type 1 interferons produced by infected cells induces an increase in the expression of MHC class I molecules on surrounding cells, which increases the presentation of viral antigen in infected cells to newly generated virusspecific $\mathrm{CD} 8^{+} \mathrm{CTL}$ of the adaptive immune response while simultaneously protecting noninfected cells from indiscriminate lysis by activated NK cells (Biron, 1999).

\section{CTL and NK cells utilize similar mechanisms to mediate cellular cytotoxicity.}

NK cells and CTL lyse infected target cells by perforin and granzyme mediated cytotoxicity (Shi et al., 1997; Trapani et al., 1999). Granzymes are serine esterases that proteolytically cleave and activate members of the interleukin $1 \beta$ converting enzyme (ICE)/ ced3 "cell death proteases" inducing an intracellular signaling cascade resulting in apoptosis (Shi et 
al., 1997). Perforin is another granule-associated protein whose monomers self-associated into a membrane pore-forming complex that allows granzyme access into the target cell cytosol (Shi et al., 1997; Trapani et al., 1999). The granules of cytotoxic immune cells also contain the antibacterial protein granulysin, which is believed to play a role against intracellular bacteria such as mycobacteria (Stenger et al., 1998). NK cells and CTL also produce IFN- $\gamma($ Gregg and Bernstein, 1997), which activates the antigen presenting cell (APC) functions of local tissue macrophages, and induces them to produce a battery of pro-inflammatory cytokines such as IL-12, IL-1, TNF$\alpha$, and IL-6 (Creery et al., 1996).

\section{Innate immunity to viral infection both presages and precipitates adaptive $T$ cell immunity.}

In the first few days of a virus infection, before the generation of a virus-specific CTL response, NK cells, activated by type 1 interferon produced by infected cells, subsequently produce IFN- $\gamma$, inducing macrophage local tissue macrophage activation and cytokine production in paracrine fashion (Biron, 1999). Macrophage TNF- $\alpha$ and especially IL-12, reciprocally activate the NK cells, inducing further IFN- $\gamma$ production and thereby creating a positive feedback cycle of inflammatory mediators (Carson et al., 1999). Inflammatory cytokines, and possibly debris of damaged, infected cells induces immature resident dendritic cells to begin maturation and migration via the tissue lymphatic vessels into the afferent lymphatics of local draining lymph nodes (Moodycliffe et al., 2000). Dendritic cells, the premier APC of the immune system, are highly endocytic cells of hematopoeitic origin that avidly accumulate protein antigens from their local tissue microenvironment, during their immature phase (Moodycliffe et al., 2000). Maturation of dendritic cells, mediated by elevated local inflammatory cytokine levels, causes the cessation of antigen uptake, and the commencement of antigen processing within endolysosomal vesicles, followed by presentation of antigenic peptides 
in association with cell surface MHC class II molecules coincident with migration to local lymphoid tissue (Moodycliffe et al., 2000). Dendritic cells are also readily infected by viruses, and endogenous viral antigens are enzymatically processed in the cytosol for presentation in association with MHC class I molecules (Muller et al., 2000). Dendritic cells arrive at the draining lymph node and encounter naïve T cells in the paracortex (Moodycliffe et al., 2000). Interdigitating dendritic cells are also located within the node from the region of the paracortex to the medulla in the $\mathrm{T}$ cell zones surrounding the $\mathrm{B}$ cell follicles, and also serve to present peptides processed from antigens that have either flowed freely in the afferent lymphatic vessel to the node, or that is donated on membrane vesicles pinched off from immigrant dendritic cells from the inflamed tissues (Russo et al., 2000).

\section{Dendritic cells are the premier antigen presenting cells in priming a CTL response.}

Dendritic cells are unique among APC in having the ability to stimulate naïve $\mathrm{CD} 4{ }^{+}$and $\mathrm{CD}^{+} \mathrm{T}$ cells (Banchereau et al., 2000). T cells recognize fragments of peptide antigens, known as epitopes, expressed on APC cell surfaces in association with the specialized cell surface antigen presentation complexes; HLA in humans and H-2 antigens in mice (Lambrecht et al., 2000). A peptide fragment expressed on an MHC molecule is called an epitope. When an epitope is specifically recognized by a $\mathrm{T}$ cell, and induces a $\mathrm{T}$ cell response, it is called a 'cognate epitope'.

\section{Generation and presentation of viral peptide epitopes.}

Peptide antigen derived from exogenous sources (those that are phagocytosed from outside the dendritic cell) are processed in endolysosomal vesicles wherein protein antigen is fragmented by acid dependent proteases (cathepsins), and fragments 18-22 amino acids long can become associated with MHC class II molecules within the endosome, and subsequently shuttled 
to the cell membrane for presentation to the $\mathrm{T}$ cell receptor (TCR) of $\mathrm{CD} 4^{+}$helper $\mathrm{T}$ cells (Chapman et al., 1998). Self or viral peptide antigen derived from the cytosol is enzymatically cleaved by the large multicatalytic proteosome (LMP), and peptide fragments are then transported into the lumen of the endoplasmic reticulum (ER) by the transporter associated with antigen presentation (TAP 1 and 2) heterodimer (Hammond et al., 1993). Within the ER, antigenic peptides are trimmed by aminopeptidases to fragments of roughly 8-12 amino acids, and are bound within the binding cleft of MHC class I molecules, which are themselves associated with $\beta 2$ microglobulin (York et al., 1999). The resultant heterotrimeric complex is then transported to the cell surface by way of the golgi apparatus (York et al., 1999). Dendritic cells are also capable of presenting exogenous, phagocytosed antigen in the context of MHC class I molecules, a phenomenon known as cross-presentation, thus allowing the priming of $\mathrm{CD} 8^{+}$cells to antigens that would under conventional circumstances be presented to $\mathrm{CD} 4^{+}$cells (Castellino et al., 2000; Hamano et al., 2000).

\section{Ligation of co-stimulatory molecules on APC by responder $\mathrm{T}$ cell receptors potentiates, augments, and maintains $T$ cell responses.}

Virus-specific $\mathrm{CD}^{+}$and $\mathrm{CD} 8^{+} \mathrm{T}$ cells interacting with cognate MHC-peptide via their TCR are initially induced to proliferate. Priming of naïve $\mathrm{CD} 4^{+}$and $\mathrm{CD} 8^{+} \mathrm{T}$ cells is thought of in terms of the two-signal model. Upon TCR ligation, a signal is transduced by the TCR-associated CD3 complex, providing the first and most important signal toward activation. In the absence of a second, co-stimulatory signal, the $\mathrm{T}$ cell is generally rendered anergic, or refractory to further stimulation, and thus unable to respond to cognate antigen (Swartz, 1997). Mature dendritic cells, like activated macrophages and B cells, provide the required costimulatory signal via cell surface expression of B7.1 and B7.2, which are the ligands of the T cell surface molecule CD28 (Andreasen et al., 2000). Ligation of CD28 by dendritic cell B7 enhances the TCR mediated 
signal to fully activate the naïve $\mathrm{T}$ cell (Vieira et al., 2000). While the mature dendritic cell constitutively expresses surface B7, interaction of the APC surface receptor CD40 with the T cell surface CD40 ligand (CD40L) found predominately on the surface of $\mathrm{CD} 4^{+} \mathrm{T}$ cells, greatly increases the APC expression of B7 (Andreasen et al., 2000), as well as the secretion of T cell stimulatory cytokine IL-12 (Snijders et al., 1998). Because of the enhancement of APC costimulatory activity following CD40 ligation by $\mathrm{CD}^{+} \mathrm{T}$ cell CD40L, it is believed that most $\mathrm{CD}^{+} \mathrm{T}$ cell responses require that $\mathrm{CD} 4^{+}$cells first interact with the dendritic cell to fully condition APC co-stimulatory activity (Lefrancois et al., 1999; Andreasen et al., 2000). However, $\mathrm{CD}^{+}$cells are not absolutely required to generate $\mathrm{CTL}$ responses in some massive systemic virus infections, such as infection with LCMV, or to induce CTL responses to nonreplicating peptide antigen in transgenic mice with $\mathrm{CD} 8^{+} \mathrm{T}$ cells expressing invariant TCR (Lefrancois et al., 1999). It is believed that the presence of inflammatory cytokines causes dendritic cells to acquire sufficient co-stimulatory ability to prime a CTL response without the need of prior CD40L-CD40 interaction (Kim et al., 1998; Andreasen et al., 2000), although there is evidence that $\mathrm{CD} 40 \mathrm{~L}$ expressed on $\mathrm{CD} 8^{+}$cells also interacts with $\mathrm{CD} 40$ on dendritic cells to upregulated B7 expression (Andreasen et al., 2000). The requirement of co-stimulation to fully activate a naïve $\mathrm{CD} 8^{+} \mathrm{T}$ cell is, however, variable, depending on the strength of the TCRMHC/peptide complex interaction (Andreasen et al., 2000), the number of cognate MHC-peptide complexes on the surface of the APC (Wherry et al., 1999), the local cytokine milieu (Kim et al., 1998), and the prior antigen experience of the T cell (naïve vs. memory). One of the effects of CD28-B7 interaction is to augment the production of IL-2, the premier T cell proliferation and activation cytokine. This augmentation is mediated by the stabilization of IL- 2 mRNA in CD4 ${ }^{+} \mathrm{T}$ cells, which are the major IL-2 producing cells, and to a lesser extent in $\mathrm{CD} 8^{+}$cells (McAdam et 
al., 1998). Evidence also exists for other co-stimulatory molecules on the surface of the APC, possibly related to B7.1 and B7.2, but their role in $\mathrm{T}$ cell responses, if any, are as yet not known (Swallow et al., 1999).

\section{$\mathrm{CD8}^{+} \mathrm{T}$ cells are induced to proliferate, emigrate and differentiate after TCR ligation of cognate antigen and restricting MHC molecules.}

Responder T cells initially proliferate, creating progeny cells bearing identical TCR, and thus having identical antigen specificity. Murine $\mathrm{CD} 8^{+} \mathrm{T}$ cells can proliferate at a maximal rate of 3 times per day in response to LCMV infection (Zimmerman et al., 1999), although the rate for other $\mathrm{T}$ cell responses likely varies as a function of the conditions that also influence the magnitude of the response (Bousso et al., 1999). Proliferating antigen specific $\mathrm{CD} 8^{+} \mathrm{T}$ cells may remain in the lymph node, or may emigrate from the lymph node via the efferent lymphatic vessels to continue proliferating and differentiating in the periarterilolar sheath of the splenic white pulp (Tarazona et al., 1996). It is not certain if the proliferation of recently activated CD $8^{+}$ T cells emigrating to the spleen requires continued stimulation in situ by APC presenting cognate antigen or is an intrinsic ability of these cells, but proliferation in the spleen believed to dramatically enhance the overall number of progeny antigen-specific $\mathrm{T}$ cells as much as 50 -fold in some models, and possible much more in some systemic virus infections (Vasseur et al., 1999). Whether in the spleen or lymph node, the $\mathrm{CD} 8^{+} \mathrm{T}$ cells eventually shift from a naïve phenotype, such as $\mathrm{CD}_{2}{ }^{+}\left(\mathrm{L}-\right.$ selectin $\left.^{+}\right), \mathrm{CD}^{+} 8^{+} \mathrm{CD} 4 \mathrm{RA}^{+}$(Doyle et al., 1999), to an effector phenotype of CD $62^{\text {lo }} \mathrm{CTLA}^{+} \mathrm{CD} 44^{+} \mathrm{CD} 45 \mathrm{RA} / \mathrm{RO}^{+}, \mathrm{CD} 25^{+} \mathrm{CD} 69^{+} \mathrm{LFA}^{+}{ }^{+}$VLA $-4^{+} \mathrm{CD} 56^{+}$ (Doyle et al., 1999). These phenotypic changes coincide with the acquired ability of the $\mathrm{CD} 8^{+} \mathrm{T}$ cell to migrate to inflamed peripheral tissue via the venous circulation, and to adhere via the LFA-1 $\left(\alpha_{x} \beta_{2}\right)$ and VLA-4 $\left(\alpha_{4} \beta_{1}\right)$ integrins to ICAM-1 and VCAM-1 expressed on the surface of endothelial cells of post-capillary venules in inflamed peripheral tissue (Parr and Parr, 2000), to 
cross the endothelial barrier to the extravascular compartment, there to mediate effector functions such as cytotoxicity and IFN- $\gamma$ and TNF- $\alpha$ production (Cerwenka et al., 1999; Trapani et al., 1999). Tissue-specific heterodimeric integrin molecules are expressed on antigenexperienced effector and memory cells dependent on the lymphoid tissue in which the naïve $\mathrm{T}$ cell precursor first encountered its cognate antigen, and governing the tissue to which the antigen experienced cell will most likely migrate (MacKay et al., 1996; Tarazona et al., 1996). Thus, the progeny of naïve $\mathrm{CD} 8^{+} \mathrm{T}$ cells encountering their cognate antigen on the MHC class I molecules of dendritic cells in lymph nodes draining the skin will express $\alpha_{4} \beta_{1}$ (MacKay et al., 1996) and the cutaneous lymphocyte antigen (Santamaria et al., 1995), which are specific for the skin epithelia, as well as the LFA-1 and VLA integrins, which are specific for ligands such as VCAM-1 expressed on inflamed epithelial cells, allowing extravasation into inflamed tissues. By contrast, the progeny of naïve $\mathrm{CD} 8^{+} \mathrm{T}$ cells encountering cognate viral peptide in the mesenteric lymph nodes, Peyer's patches, or other mucosa-associated lymphoid tissue (MALT) will express $\alpha_{4} \beta_{7}$, (MacKay et al., 1996) an integrin specific for the mucosal addressin cell adhesion molecule (MadCAM-1) (Lefrancois et al., 1999). MadCAM-1 is constitutively expressed on the endothelial cells of post-capillary venules of mucosal tissues, as well as specialized highendothelial venules of the MALT (Berlin et al., 1993). Mucosally primed CD8 ${ }^{+}$cells may also express $\alpha_{\mathrm{E}} \beta_{7}$, an integrin specific for E-cadherin expressed in the epithelial cells of mucosal tissue (Lefrancois et al., 1999). The acquired effector phenotype also coincides with effector function, which in the case of $\mathrm{CD} 8^{+}$cells is perforin/granzyme or FasL mediated cytotoxicity and the production of IFN- $\gamma$, and TNF- $\alpha$, or rarely IL-4 (Harty et al., 2000). 


\section{Kinetics of the CTL response are similar for several viral infections; prima facie and heuristic evidence that proliferative characteristics of anti-viral CTL responses are conserved.}

Maximal numbers of virus-specific $\mathrm{CD}^{+} \mathrm{T}$ cells are usually reached within the first or second week post-infection (Doherty et al., 1997). Murine intraperitoneal infection with lymphocytic choriomeningitis virus (LCMV), Pichinde virus, vesicular stomatitis virus, vaccinia virus, and murine cytomegalovirus (MCMV) elicited maximal virus-specific CTL frequencies by days 7-8 post-infection as determined by limiting dilution analysis (LDA) (Selin et al., 1996). Oral infection of immunocompetent mice with reovirus and murine rotavirus was usually cleared no later than day 10 post-infection (Barkon et al., 1996), which would occur after the maximal CTL response. Similar rates of maximal CTL response generation also occur for influenza infection (Jameson et al., 1998) and vesicular stomatitis virus infection of humans (Sharp et al., 1992), although Epstein Barr virus infection of human patients induces maximal CTL responses

usually 2 weeks after presentation of patients with acute infectious mononucleosis (Lynne et al., 1998), and the peak of the cell mediated response in measles infection is coincident with the development of the characteristic rash 2 to 3 weeks after infection (Mongkolsapaya et al., 1999). Despite some variability in the timing of virus-specific CTL responses, conserved features are a rapid increase in the frequency of virus-specific cells that is in the main positively correlated with the degree to which the virus replicates in the host tissue, the subsequent death of the majority of the progeny CTL following resolution of the infection (Lynne et al., 1998), and the maintenance of memory CTL (mCTL) which are antigen-experienced and have a lowered threshold of required stimulation and co-stimulation for reactivation despite being otherwise quiescent with regard to proliferation or effector function in the absence of antigen (Lynne et al., 1998). 


\section{Quantification by LDA of CTL response magnitude, and cotemporaneous generation of the 'bystander' phenomenon.}

Proliferative responses of virus-specific CTL following infection have classically been quantified directly by LDA or indirectly by determining the effector to target ratio required for measurable virus-specific killing of infected target cells by ${ }^{51} \mathrm{Cr}$ release assay (Doherty et al., 1997). The number of cells determined to be antigen-specific by these assays is dependent on the number of virus-specific cells that are capable of proliferating in vitro and the number of resulting progeny cells that can assume CTL effector function. By LDA, the frequency of virusspecific cells was determined to increase often a thousand-fold following a primary virus infection (Doherty et al., 1997), and this expanded CTL population was found to decrease by less than a factor of ten following the resolution of the virus infection (Selin et al., 1996). For example, LDA of splenocytes from LCMV and VV infected mice showed a frequency of virusspecific CTL at day 8 post-infection of approximately 1 in 30 and 1 in 70, respectively, decreasing to approximately 1 in 200 to 1 in 500 within one month and there remaining constant up to one year post-infection (Selin et al., 1996). However, the number of proliferating CD $8^{+}$ cells recoverable during a virus infection is usually far in excess of the number of virus-specific CTL that can be accounted for by LDA (Doherty et al., 1997). This led to speculation that the unaccountable proliferation was due to the proliferative expansion of $\mathrm{CD}^{+}$cells of various other specificities, non-specifically activated in the environment of a massive ongoing immune

response to the infecting virus. This could occur due to cross-reactivity of some $\mathrm{CD} 8^{+}$cells with non-cognate virus antigen that 'looks' structurally similar to cognate antigen to the TCR of cross-reactive T cells or due to cytokine (especially IL-2) driven antigen-independent proliferation of mCTL due to their decreased activation threshold (Hou et al., 1994). These nonspecific responses were termed "bystander" responses, and were believed to underlie the 
observation that the onset of a number of autoimmune conditions was apparently linked to prior viral infection (Horwitz and Sarvetnick, 1999). It was eventually demonstrated using naïve or antigen -experienced transgenic $\mathrm{CD} 8^{+} \mathrm{T}$ cells bearing invariant TCR specific for a known LCMV antigen, adoptively transferred into vaccinia virus-infected or Listeria monocytogenesinfected non-transgenic recipients, that bystander responses did occur, in this case inducing antiLCMV CTL effector function by the transgenic T cells (Ehl et al., 1997). However, the induced bystander CTL responses were very weak. Thus, the cause of the massive 'bystander' response seen following LCMV infection, and other virus-induced $\mathrm{CD}^{+}$responses, had to be reevaluated.

\section{Reevaluation of quantification by LDA of CTL response magnitude, and cotemporaneous reassessment of the 'bystander' phenomenon.}

Virus infections can cause a massive expansion of $\mathrm{CD} 8^{+} \mathrm{T}$ cells. Infection of $\mathrm{C} 57 \mathrm{BL} / 6$ with LCMV has been shown to cause a proliferative expansion of $\mathrm{V} \beta 8.1^{+} \mathrm{CD} 8^{+} \mathrm{T}$ cell population from $3 \%$ to $16 \%$ of splenic $\mathrm{CD} 8^{+}$cells, and to increase the overall number of $\mathrm{CD} 8^{+}$cells in the spleen from about $20 \%$ of total splenic mononuclear cells to more than $35 \%$ (Lin and Welsh, 1998). Intranasal infection of C57BL/6 mice with influenza A results by day 5 post-infection in a 5 -fold increase in the percentage of mediastinal lymph node $\mathrm{CD} 8^{+}$cells in the $\mathrm{S}$ and G2/M stage of the cell cycle as compared to cells from non-infected controls Tripp et al., 1995). Furthermore, up to $20 \%$ of splenic $\mathrm{CD} 8^{+}$cells are found to be proliferating in the spleens of influenza infected mice by day 5. LDA analysis of splenocytes from influenza infected mice revealed an unexpectedly low frequency of just 1 virus- specific pCTL per $600 \mathrm{CD} 8^{+}$cells at the time of the maximal CTL response, and preceding the clearance of replicating virus by 2 days (Tripp et al., 1995). Influenza infection caused a skewing of the V $\beta$ repertoire of infected mice in favor of $\mathrm{V} \beta 8^{+} \mathrm{CD} 8^{+}$cells, and virus-specific CTL were shown to express predominantly V $\beta 8.3$ 
and to a lesser extent V $\beta 8.1$ (Doherty et al., 1997), but the increase in $\mathrm{V} \beta 8^{+} \mathrm{CD} 8^{+}$cells was paradoxically not in agreement with the results of the LDA.

Symptomatic human infection with EBV, 'acute infectious mononucleosis', is a lymphocytosis in which the absolute number of circulating $\mathrm{CD}^{+}$cells increases 4 to eight times over that of healthy controls (Lynne et al., 1998). These cells show an activated phenotype with expression of HLA-DR, an MHC class II molecule expressed on activated human T cells, and the low molecular weight isoform of CD45 (CD45RO) expressed by antigen-experienced T cells (Silins et al., 1998). The expansion of $\mathrm{CD}^{+}$cells is limited to one or a few V $\beta$ subpopulations as determined by flow cytometric staining for $\mathrm{V} \beta$ repertoire (Silins et al., 1998). LDA for pCTL specific for EBV lytic cycle proteins give an estimate of virus specific CTL among total CD8 ${ }^{+}$ cells among peripheral blood mononuclear cells (PBMC) of only $1 / 100$ to $1 / 500$ (BenningerDoring et al., 1999). This pattern of massive virus-driven expansion of CD8 ${ }^{+}$cells in infected hosts far in excess of the number of virus-specific pCTL detectable by LDA, yet still demonstrating TCR V $\beta$ repertoire skewing in favor of $\mathrm{V} \beta$ phenotypes utilized by virus-specific CTL has also been seen in macaques infected with simian immunodeficiency virus (SIV) (Wilson et al., 1998), and in humans infected with HIV (Cabonari et al., 1999) and measles (Mongkolsapaya et al., 1999). By way of example, in a human measles infection, peripheral blood $\mathrm{CD}^{+} \mathrm{TCR} \mathrm{V} \beta^{+}$populations of acutely infected hosts expand up from 3 to 10 -fold over healthy controls (Mongkolsapaya et al., 1999). While peripheral blood CD8 ${ }^{+}$cells from infected subjects can be induced to mediate virus-specific cytotoxicity by ${ }^{51} \mathrm{Cr}$ release assay, depletion of the $\mathrm{CD}^{+}$cells expressing the expanded TCR V $\beta$ phenotype by magnetic sorting abrogates the ability of the $\mathrm{CD}^{+}$cells to kill virus-infected targets, suggesting that expanded $\mathrm{CD} 8^{+} \mathrm{TCR} \mathrm{V} \beta$ subpopulations are virus-specific (Mongkolsapaya et al., 1999). Furthermore, cloning of PCR 
amplified cDNA generated from reverse transcription of TCR beta chain mRNA indicated that the expanded V $\beta$ subpopulations were dominated by a handful of clones in infected patients, with some clones accounting for more than $60 \%$ of all cells from an expanded V $\beta$ subpopulation (Mongkolsapaya et al., 1999). This was more evidence to indicate that the massive expansion of $\mathrm{CD}^{+} \mathrm{T}$ cells following infection was mainly virus-specific, as a true bystander response would be expected to cause a broadly polyclonal, stochastic expansion.

\section{Quantification of CTL response magnitude by IFN- $\gamma$ ELISPOT, intracellular cytokine staining, and MHC class I/peptide tetramers staining.}

The discordance between the quantity of virus-specific CTL as determined by LDA and the observed dramatic increases of activated $\mathrm{CD} 8^{+} \mathrm{T}$ cells following virus infection was finally put to rest by assays looking at other CTL effector functions that, unlike the LDA, were not dependent on proliferation or cytotoxicity. Two new techniques, the determination of virusspecific cells by intracellular IFN- $\gamma$ (or TNF- $\alpha$ ) production by flow cytometry, and the determination of IFN- $\gamma$ secretion by ELISPOT analysis, allowed more accurate quantitation of virus-specific effector T cells. Another flow cytometry technique, which relied on knowledge of the cognate viral epitopes and the restricting MHC class I alleles of the responder CTL, was the peptide-MHC class I tetramer staining. For viruses the epitopes of which were already mapped, such as LCMV, the number of virus-specific CTL determined by IFN- $\gamma$ staining or ELISPOT was found to closely approximate tetramer-positive $\mathrm{CD} 8^{+}$cells, and to be more than 10 -fold greater than the number of virus-specific cells determined by LDA, thus accounting for almost all the 'bystander' response (Murali-Krishna et al., 1998). By intracellular IFN- $\gamma$ staining of $\mathrm{CD} 8^{+}$splenocytes in mice infected with LCMV, Butz and Bevan (1998) showed that virtually all the activated $\mathrm{CD}^{+}$cells, which can account for more than $50 \%$ of all $\mathrm{CD} 8^{+}$cells in the spleen, were LCMV-specific. Similar results were obtained by IFN- $\gamma$ specific ELISPOT analysis of 
$\mathrm{CD}^{+}$splenocytes from LCMV-infected mice. $\mathrm{H}-2 \mathrm{k}^{\mathrm{d}}$ tetramers complexed with the known immunodominant LCMV epitopes showed almost all the activated CD $8^{+}$cells from LCMV infected Balb/c mice were specific to a single peptide epitope (van der Most et al., 1998), whereas in $\mathrm{C} 57 \mathrm{BL} / 6$ mice, the majority of activated $\mathrm{CD}^{+} \mathrm{T}$ cells could be accounted for by tetramers using a panel of 4 epitopes (van der Most et al., 1998). Class I tetramers complexed with epitopes from the gag and pol proteins of HIV have also shown $\mathrm{CD}^{+} \mathrm{T}$ cell expansions in the peripheral blood of infected humans are virus-specific (Gary et al., 1999), and similar results have been found in SIV infected macaques (Donahoe et al., 2000), Tetramer staining or assessment of IFN- $\gamma$ production have given revised frequencies of virus-specific CTL ranging from 20 to 500 times greater than those previously determined by LDA (Murali-Krishna et al., 1998). While tetramer staining and the quantification of IFN- $\gamma$ producing $C D 8^{+}$cells have been considered essentially equivalent in detection efficacy, there are some differences regarding application. Tetramer staining is only feasible when the restricting $\mathrm{H}-2$ or MHC molecule and also the cognate epitopes are known.

\section{Advantages of MHC tetramer staining.}

If the cognate epitopes are known, tetramer staining allows quantitation of the magnitude of the CTL response to each epitope of an infectious agent. Tetramer staining also allows concomitant assessment of cell surface phenotypic markers such as the CD45 isoforms, surface integrins, and activation markers of memory, effector, and naive T cells, and does not rely on the ability of the $\mathrm{CD}^{+}$cell to mediate effector function. $\mathrm{V} \beta$ repertoire can also be assessed, as apparently there is no steric hindrance between $\mathrm{mAb}$ reacting to conserved structures of the $\mathrm{V} \beta$ chain and the tetramers/epitope complex (Busch and Pamer, 1998). Intracellular IFN- $\gamma$ staining also allows assessment of surface phenotypic markers such as the $\mathrm{V} \beta$ repertoire of virus-specific 
cells. This method is dependent on the ability of the cells to mediate an effector function - IFN- $\gamma$ production- although not cytotoxicity or proliferation. This method also has the advantage of not requiring knowledge of the cognate epitope or the restricting H-2 or HLA allele.

\section{Advantages of intracellular interferon- $\gamma$ staining.}

Without some degree of prior cell sorting, IFN- $\gamma$ ELISPOT analysis does not allow correlation of effector cell number with cell phenotype, as $\mathrm{CD} 4^{+}$cells and NK cells also may produce IFN- $\gamma$ in response to virus, but like intracellular IFN- $\gamma$ staining, ELISPOT analysis does not require knowledge of the cognate epitopes and restricting H-2/HLA alleles. ELISPOT analysis has also been shown to be far more sensitive than the flow cytometric techniques in detecting extremely small numbers of effectors (<1:1000) (Murali-Krishna et al., 1998). Although this is hardly a problem for most acute virus infections, it may have application in quantifying long-term antiviral memory.

\section{Decline of the CTL response with resolution of infection, maintenance of memory and 'rapid effectors'.}

The vast majority of the virus specific CTL generated in the acute response is predisposed to die by apoptosis (Selin et al., 1996). Following infection in C57BL/6 mice, the frequency of LCMV, Pichinde virus, vaccinia virus, and MCMV virus-specific pCTL was found by LDA to decline 4 to 10 fold within 1 month following infection, and to decrease steadily, though less rapidly, over the next several months (Selin et al., 1996). Similar kinetics were observed by LDA for the decrease of splenic virus-specific pCTL from mice infected with Sendai virus, Influenza A, and respiratory syncytial virus (RSV) (Doherty et al., 1997). In humans, the TCR V $\beta$ perturbations of peripheral blood $\mathrm{CD} 8^{+} \mathrm{T}$ cell population corrected to "baseline" (not significantly different from the V $\beta$ repertoire of healthy controls) within 6 months for EBV acute infectious mononucleosis (Silins et al., 1998) and within 4 months for 
measles (Mongkolsapaya et al., 1999). Thus, without persistence of replicating virus, the frequency of virus-specific cells dramatically decreases. It was believed that as activated effector cells die (Ishigami et al., 1998) or become quiescent memory cells (Opferman et al., 1999), that the frequency of remaining virus specific cells would approach the frequency detectable by LDA, especially over a period of months or years post-infection. Thus, tetramer staining or intracellular IFN- $\gamma$ staining, both of which rely on flow cytometry, would lack the sensitivity to detect virus-specific cells without first a period of reactivation and proliferation. In human subjects having recovered from influenza A infection several months previously, $\mathrm{CD} 8^{+}$cells in the peripheral blood were found to be able to produce IFN- $\gamma$ within 6 hours of exposure to influenza infected syngenic APC by ELISPOT (Lalvani et al., 1997). The frequency of IFN- $\gamma$ producing $\mathrm{CD}^{+}$cells was found to be 7 to 10 fold greater than that detectable by LDA, although still too few to effectively enumerate by intracellular IFN- $\gamma$ staining or tetramer staining. These findings imply that following the apoptotic contraction of virus-specific CTL numbers after resolution of acute infection, a population of virus-specific $\mathrm{CD} 8^{+}$memory cells that can rapidly mediate effector function (IFN- $\gamma$ production) persist at a frequency several times greater than the pCTL detectable by LDA, which of course means these cells lack the ability to proliferate (Alexander-Miller et al., 1996), or mediate cytotoxicity, or both. That virus-specific cells that cannot proliferate or kill virus-infected targets, yet can produce cytokines, do exist in large numbers must account for the great majority of the $\mathrm{CD} 8^{+} \mathrm{T}$ cell expansion seen following acute virus infection (Lalvani et al., 1997). That this condition persists following the post-resolution apoptotic contraction phase raises the question as to whether some effector cells that lack proliferative and/or cytotoxic potential escape from apoptosis? 


\section{Loss of CTL with continuous antigenic stimulation can be limited by anti-apoptotic and $T$ cell-tropic cytokines.}

$\mathrm{CD}^{+}$cells can be induced to proliferate, and even to mediate effector function (IFN- $\gamma$ production) before acquiring a proliferation-incompetent anergic state, yet such cells may persist in vivo for extended periods (Dubois et al., 1998). Adoptive transfer of transgenic CD8 ${ }^{+}$cells with a $\mathrm{V} \beta 5^{+} \mathrm{TCR}$ into congenic recipient mice expressing a provirus encoded cognate antigen resulted in rapid activation and proliferation of the transferred cells (Dubois et al., 1998). Progeny transgenic cells had an effector phenotype, but rapidly became proliferation incompetent even in the presence of IL-2, yet they were able to produce IFN- $\gamma$ on cross-linking of $\mathrm{CD} 3$ complexes by anti-CD3 $\alpha$ monoclonal antibodies. These cells persisted in vivo with a $\mathrm{t}_{1 / 2}$ of 5 days, although this was in the continued presence of the cognate antigen that induced their inactivation. Such models of 'high dose tolerance' with respect to $\mathrm{CD} 8^{+} \mathrm{T}$ cell effector function using TCR transgenic $\mathrm{CD} 8^{+}$cells provide useful tools for understanding stereotypical aspects of CTL responses also seen in virus infections. For instance, activated, proliferation-incompetent CTL were shown to have decreased expression of Bcl-2, an apoptosis-inhibiting factor the downregulation of which is seen in anti-viral CTL at the resolution of a viral infection (Grayson et al., 2000). Cytokines such as IL-2, IL-4, IL-7, and IL-15 bind to receptors that share common transmembrane signal transducing $\gamma$ chains. These cytokines act in paracrine fashion to increase Bcl-2 levels in activated $\mathrm{T}$ cells, thus preventing apoptosis. Type 1 interferon also has a role in preserving effector cells from apoptosis by a mechanism independent of Bcl-2 (Mitchell et al., 1999). In the absence of anti-apoptotic factors, especially the $\mathrm{CD}^{+} \mathrm{T}$ helper cell cytokine IL-2, most $\mathrm{CD}^{+}$effector cells are fated to die (Grayson et al., 2000). This is best exemplified in LCMV infection of CD4 gene "knock out" (CD4KO) (von Herrath et al., 1996) or IL-2KO mice (Bachmannl et al., 1995). C57BL/6 mice infected with LCMV mount rapid and protective CTL 
responses to the epitopes GP33 and NP396 (van der Most et al., 1998). By contrast, LCMV infection of CD4KO mice resulted in chronic infection (von Herrath et al., 1996). Tetramer epitope staining of $\mathrm{CD}^{+}$cells during the first week post-infection showed induction of GP33 and NP396 specific CD8 ${ }^{+}$cells with comparable kinetics for both wild type and CD4KO mice, but apoptosis soon claimed the NP396 specific and most of the GP33 specific cells in the CD4KO mice, with those few GP33 specific cells remaining being unable to proliferate (Gallimore et al, 1999): Antiviral CD ${ }^{+} \mathrm{T}$ cells responses were inducible in the absence of CD $4^{+}$ $\mathrm{T}$ helper cells, but the assistance of the $\mathrm{CD} 4^{+}$cells was required to maintain the response. Studies using IL-2KO mice indicated that $\mathrm{CD}^{+}{ }^{+} \mathrm{T}$ cells were dispensable, but IL-2 was essential in the generation and maintenance of antiviral CTL responses (Bachmann et al., 1995). It is thought that $\mathrm{CD}^{+} \mathrm{T}$ cell independent $\mathrm{CD} 8^{+}$responses can occur because APC B7 co-stimulation through the $\mathrm{CD}^{+} \mathrm{T}$ cell $\mathrm{CD} 28$ molecule allows the responding $\mathrm{CD} 8^{+} \mathrm{T}$ cell to produce sufficient IL- 2 to proliferate (Gajewski et al., 1995), but the very process of continuous activation through the TCR renders the progeny $\mathrm{T}$ cells prone to apoptosis, a process known as activation induced cell death (AICD). AICD can be prevented in the presence of exogenous CD4 ${ }^{+}$T helper cytokine IL2 in quantities far in excess of that produced by $\mathrm{CD}^{+}$cells (Deeths et al., 1999). It is therefore very significant that virus-specific $\mathrm{CD}^{+}$cells proliferate 10 to 100 fold less than $\mathrm{CD} 8^{+}$cells (Whitmire and Ahmed, 2000), and that most of the T cells isolated at sites of inflammation are $\mathrm{CD}^{+}$effector cells, as many of the activated $\mathrm{CD} 8^{+}$cells may not receive sufficient $\mathrm{CD} 4^{+}$ cytokine support to prevent apoptosis even with a normal $\mathrm{CD} 4^{+}$response (Whitmire and Ahmed, 2000). Thus, the range and quantity of anti-apoptotic cytokines in the local milieu of effector $\mathrm{CD}^{+}$cells determines future potential: cell death, proliferation and cytotoxicity incompetent 
IFN- $\gamma$ producing effector function, or fully replication competent mCTL that are detectable by LDA.

\section{Anti-viral CTL can be generated against epitopes derived from numerous viral proteins.}

CTL responses are generated against one or more epitopes for any given infectious agent. In a virus infection, epitopes can be peptide fragments of viral structural proteins that form the infectious viral particle, such as HA and NA from influenza (Gianfrani et al., 2000) or HA from reovirus (Finberg et al., 1981), capsid proteins VP5, VP7 (Heath et al., 1997) VP3 and VP6 (Franco et al., 1994) from rotavirus, and nucleoproteins from the viral core in influenza (Kees and Krammer, 1984), parainfluenza (Cole et al., 1995), and LCMV (van der Most et al., 1998). Non-structural viral proteins, which are involved in replication of the virus but not incorporated into the complete virion, such as reovirus $\sigma 1 \mathrm{NS}$ (Hoffman et al., 1996) and human papilloma virus (HPV) early proteins E6 and E7 (Bourgault et al., 2000) can also be the source of antigenic epitopes for CTL responses. It has been suggested that CTL responses are stereotypically generated against invariant viral peptides (Kees and Krammer, 1984), whereas for example influenza may undergo antigenic drift, minor changes due to in the protein sequence of the HA and NA molecules due to mutations in the genomic RNA (Lambkin et al., 1994), or antigenic shift due to reassortment of genome RNA segments between related heterologous influenza viruses co-infecting the same host and thus rendering influenza virions able to escape previously elicited humoral immunity (Castrucci et al., 1993), the influenza specific CTL recognize epitopes from conserved NP proteins, which make virions unable to escape the CTL recall response (Kees and Krammer, 1984). However, this phenomenon does not seem axiomatic, and the factors governing the immunogenicity of an epitope in eliciting the anti-viral CTL response seem more related to the quantity of that epitope that can be stably processed and presented on 
the MHC molecules of an APC (Wherry et al., 1999), and the ability of the $\mathrm{CD} 8^{+} \mathrm{T}$ cell repertoire to recognize the MHC epitope complex, than what role the viral protein plays in viral replication or structure. While the ability of some mCTL to recognize conserved epitopes may give a selective advantage in recall responses over $\mathrm{mCTL}$ that recognize epitopes prone to mutation, CTL do not seem to play much role in protection from re-infection as compared to circulating antibody. For a number of viruses, protection from re-infection is mediated by neutralizing and even non-neutralizing antibody, and it is unlikely much selection would occur on the virus-specific mCTL population in the face of extant protective humoral immunity.

\section{Anti-viral CTL responses are usually, but not exclusively, generated against a panel of distinct epitopes.}

The virus-specific response to a given virus is often reactive to more than one viral peptide epitope, giving a graded response pattern where one or a few epitopes are strongly responded to, and others are responded to various lesser degrees, so-called immunodominant and subdominant epitopes. Factors possibly influencing relative immunodominance and subdominance of epitopes are the efficiency of viral protein fragmentation by host proteases both at the level of the LMP in the cytosol and exopeptidases resident in the ER, the efficiency of transportation of potential epitopes into the ER via the TAP heterodimer, the strength of the interaction of the epitope with MHC class I alleles which directly relates to the number of peptide MHC class I complexes on the surface of the APC and the time those complexes remain stable. Additionally, $\mathrm{CD} 8^{+} \mathrm{T}$ cell dependent factors include the frequency of available naïve precursor CTL specific for a given epitope (Cao et al., 1996), and the strength of the recognition of a pCTL with a peptide-MHC class I complex. For example, there are 2 class I $\left(\mathrm{H}-2 \mathrm{~K}^{\mathrm{d}}\right)$ restricted epitopes from the hemagglutinin (HA) molecule of influenza A/Japan/57 generated in infected Balb/c mice, one of which is strongly immunodominant although both are processed and 
presented to comparable degrees (Cao et al., 1996). The initial precursor frequencies of naïve $\mathrm{CD} 8^{+}$cells that can respond to each epitope have been shown to be different, thus the magnitude of the response to the HA epitopes are dramatically different although clones of CTL specific for each epitope show equal ability on a per cell basis to kill virus infected target cells in vitro. Epitopes which do not elicit any immune response are known as cryptic epitopes, and may be due to 'holes' in the repertoire of possible responder $\mathrm{CD} 8^{+} \mathrm{T}$ cells (Cao et al., 1996), or conditional presentation by APC dependent on the degree of APC activation.

The panel of epitopes recognized by an elicited CTL response to a given virus or intracellular bacteria has been found to be stable with regard to secondary (recall) mCTL responses. For example, Listeria monocytogenes (Lin and Welsh, 1998) elicits CTL responses in mice to a panel of immunodominant and subdominant epitopes. These graded strength of the CTL response to immunodominant and subdominant epitopes is preserved among $\mathrm{mCTL}$, and the same pattern of response is seen on reinfection. Further, if mice are infected initially with L. monocytogenes mutants without the immunodominant epitopes, the subdominant responses are not made concomitantly stronger, and on secondary infection with wild type L. monocytogenes, the immunodominant epitopes elicit a CTL response of greater magnitude than the memory response against the subdominant epitopes (Busch et al., 1998; Vijh and Pamer, 1997). This indicates immunodominant epitopes do not out-compete subdominant epitopes, a finding that explains the stability of the virus-specific mCTL repertoire following subsequent reinfection. As the magnitude of the virus-specific mCTL population has been shown to be most dependent on the size of the initial proliferative response during the preceding viral infection (Hou et al., 1994), and the size of the proliferative response has been shown to be most directly related to the amount of cognate epitope expressed by APC (Wherry et al., 1999) and the size of the naïve 
$\mathrm{CD} 8^{+} \mathrm{T}$ cell population that can recognize the cognate epitope (Cao et al., 1996), it is no surprise that the relative strength of the responses for the various immunodominant and subdominant responses would be seen in subsequent recall responses (Naumov et al., 1998). Indirectly, conservation of a broad $\mathrm{T}$ cell reactivity from primary to recall responses can be inferred by the maintenance of similar TCR $\beta$ chain heterogeneity in peripheral blood mononuclear cells from EBV immune human donors responding to a subsequent EBV exposure (Coudel et al., 1999), and in mice sequentially infected with LCMV (Sourdive et al., 1998) and Listeria (Lin and Welsh, 1998). It is curious, however, that such preservation of both strong and weak epitope specific responses are maintained in vivo, but not in vitro, as cultures of $\mathrm{CD} 8^{+}$splenocytes from LCMV infected mice that are restimulated over a period of weeks progressively lose CTL responses to everything but the cognate immunodominant epitopes, a phenomenon called "repertoire focusing" (van der Most et al., 1998). It is clear, however, that in vitro culture systems may often skew CTL populations with time due to unnatural selective pressures (Arenz et al., 1997). In any event, maintenance of graded CTL responses to a plethora of epitopes is advantageous to the host, as evidenced by chronic murine hepatitis virus infection induced CNS demyelination, wherein infected C57BL/6 mice generate a massive CTL response specific for a single epitope from a viral genomic RNA associated protein (Pewe et al., 1997). The massive epitope mono-specific CTL response, as opposed to a broadly reactive response against numerous epitopes, selects among the replicating virions in the CNS for mutation in the cognate epitope. Once demyelinating disease is established in these mice, CNS viral isolates show mutation in the cognate epitope that have allowed the replicating virus to escape recognition by the wild-type virus specific CTL. Such mutant virions are not found in the CNS of infected severe combined immunodeficient (SCID) mice, indicating the selective pressure of a narrow 
CTL response has granted an advantage to mutant virions. Thus, a broad CTL response against a number of viral epitopes is advantageous to the host.

\section{Differential influences of the intestinal and systemic peripheral immune tissues upon cellular and humoral immunity}

Unique intestinal environment and cell populations may influence the selection and expansion of reovirus-specific CTL, altering the responder T cell repertoire compared to that elicited following infection in the systemic periphery.

$\mathrm{CD}^{+} \mathrm{T}$ cell responses are induced following enteric and parenteral infection of mice with reovirus. There is no evidence to definitively support or refute the selection of different

populations of $\mathrm{CD}^{+} \mathrm{CTL}$ following enteric or parenteral reovirus infection, or indeed following any virus infection. There are, however, features of the intestinal immune responses distinct from those of the systemic periphery that may exert differential selective influences on the $\mathrm{CD} 8^{+} \mathrm{T}$ cell populations induced following enteric reovirus infection.

\section{Issues of integrin-addressin mediated lymphocyte trafficking, and 'common mucosal immunity'.}

Mucosal and systemic immunity can be thought of as two separate, partially overlapping components consisting of humoral (B cell and antibody) and cell-mediated (T helper cell and CTL) elements. Mucosal immune responses are initiated within the priming sites of the mucosal associated lymphoid tissue (MALT) and provide immunity at mucosal surfaces as well as the systemic, non-mucosal periphery (Bergquist et al., 1995;Benedetti et al., 1997;Kuklin et al., 1998; Liu et al., 1998; Brennan et al., 1999; Gherardi and Esteban, 1999). Conversely, systemic immune responses generated in the peripheral lymph nodes and spleen, elicit both systemic and to a lesser extent, mucosal immunity (Premier et al., 1996; Coffin and Offit, 1998; Muir et al., 1998;Herremans et al., 1999). Mucosal humoral immunity consists primarily of immunoglobulin 
of the IgA isotype, with minor IgG and IgM components, whereas parenterally generated humoral responses are characterized primarily by immunoglobulin of the $\operatorname{IgG}$ isotypes (Weinstein and Cebra 1991; Major and Cuff, 1996). T helper cell and CTL responses can be elicited at either mucosal or systemic priming sites, although whether there are tissue specific differences in the responder T cell populations has not been determined. Antigen-specific B cells primed in the peripheral lymph nodes or spleen tend to recirculate to non-mucosal tissues such as the spleen and bone marrow (Bachmann et al., 1994). By contrast, B cells primed in the MALT priming tissues distribute themselves among all the mucosal tissues, such as the nasal, respiratory, gastrointestinal, vaginal and lacrimal mucosa, a phenomenon called 'common mucosal immunity' (Montgomery et al., 1980; Pal et al., 1996; Farstad et al., 1997). CD4 ${ }^{+}$and $\mathrm{CD}^{+} \mathrm{T}$ cells primed in either the peripheral lymph nodes or the MALT are thought to also migrate through the circulation back to the systemic or mucosal compartments, respectively (Mackay et al., 1996; Farstad et al., 1997). In practice, the tendency of T and B cells primed in the peripheral lymph nodes or MALT to redistribute along non-mucosal and mucosal segregational lines is far from absolute, and is governed by the cell surface integrins expressed by antigen-experienced lymphocytes. It has been shown that the entry of naïve lymphocytes into the priming sites of the MALT, such as the intestinal Peyer's patches and mesenteric lymph nodes, or into the spleen or peripheral lymph nodes is equivalent due to binding of 'peripheral lymph node addressin' by the lymphocyte integrin L-selectin, CD62L (Mackay et al., 1996; Farstad et al., 1997). Indeed, the majority of the T cells in the peripheral lymph nodes or Peyer's patches are naïve cells (Tietz and Hamann 1997). Antigen driven T and B cell responses result in a decrease in CD62L expression on responder lymphocytes, limiting their ability to migrate to the lymphoid tissue of the systemic and mucosal compartments indiscriminately (Chao et al., 
1997). Primed B and T cells undergo a concomitant increase in CD44, LFA-1, VLA-4, and CD2, which allows for their recruitment and extravasation at sites of active inflammatory responses (Picker et al., 1990). Mucosally primed lymphocytes and a fraction of those primed systemically also express the mucosa-specific integrin, $\alpha_{4} \beta_{7}$ (Farstad et al., 1996).

\section{Expression of cell surface integrin molecules direct subsets of activated lymphocytes to the mucosa.}

${ }_{\alpha 4} \beta_{7}$, the lymphocyte surface ligand for mucosal addressin cellular adhesion molecule, MadCAM-1, is expressed on a fraction of B and T lymphocytes primed within the peripheral lymph nodes, and on virtually all lymphocytes primed in the MALT (Kantele et al., 1997). As MadCAM-1 is normally expressed almost exclusively on the endothelium of the post-capillary venues of mucosal lamina propria (Farstad et al., 1996), and the specialized cuboidal endothelial cells of the MALT priming tissue high endothelial venules (HEV) (Brisken et al., 1993), lymphocytes primed in the mucosa are preferentially recruited back to the MALT from the circulation (Farstad et al., 1996). Expression of $\alpha_{4} \beta_{7}$ by subsets of lymphocytes primed in the periphery would lead to the prediction that these cells may also preferentially be recruited to the MALT, and this does indeed seem to be true (Farstad et al., 1997). It has been observed that antigen-experienced $\alpha_{4} \beta_{7}{ }^{+}$lymphocytes can enter all the intestinal tissues; whereas antigenexperienced $\alpha_{4} \beta_{7}{ }^{-}$cells were excluded (Farstad et al., 1996; Williams and Butcher, 1997). Naïve $\mathrm{CD}_{22} \mathrm{~L}^{+} \alpha_{4} \beta_{7}{ }^{-}$cells could enter Peyer's patches, mesenteric lymph nodes, or peripheral lymph nodes with equal efficacy but were also excluded from the lamina propria (Williams and Butcher, 1997). Antigen experienced cells $\alpha_{4} \beta_{7}{ }^{+}$and $\alpha_{4} \beta_{7}{ }^{-}$cells were ten-fold less able to enter the peripheral lymph nodes than naïve cells (Tietz and Hamann, 1997), although the spleen recruited naïve and antigen experienced cells with equal efficacy, regardless of phenotype (Williams and Butcher, 1997). Thus, the ability of $\alpha_{4} \beta_{7}{ }^{+}$cells to enter both the mucosal priming 
sites and lamina propria due to interaction with endothelium expressing MAdCAM-1provides both an explanation for the common mucosal immune system as well as the prediction that the fraction of $\alpha_{4} \beta_{7}{ }^{+}$lymphocytes primed in the peripheral lymph nodes can mediate mucosal immune function. Conversely, the fact that $\alpha_{4} \beta_{7}{ }^{+}$cells can be recruited to the spleen suggests that mucosal immune priming could also provide systemic immunity. Both these predictions hold true. For example, parenteral priming of mice with rotavirus induced rotavirus-specific $\operatorname{IgG}$ producing cells in the intestinal lamina propria, and potentiated a subsequent IgA immune response to oral challenge with rotavirus (Coffin et al., 1995). Further, parenteral LCMV infection of mice induces LCMV specific CTL in both the lamina propria and intraepithelial (IEL) compartments of the small intestine (Muller et al., 2000). Additionally, parenteral immunization with ovalbumin protein of mice adoptively transferred with transgenic $\mathrm{CD} 8^{+} \mathrm{T}$ cells bearing a $\mathrm{T}$ cell receptor (TCR) specific for an ovalbumin epitope resulted in a 700-fold increase in the number of the transgenic $\mathrm{T}$ cells recovered from the IEL compartment, and the acquisition of cytotoxic effector function (Lefrancois et al., 1999; Lefrancois et al., 2000). Conversely, mucosal immune responses to foreign organisms such as reovirus (London et al., 1987) and rotavirus (Offit and Dudzik, 1989; Feng et al., 1994), results in both mucosal and systemic immunity, in terms of both cell mediated immunity and IgA- and IgG- producing B cells.

\section{Immune responses are not necessarily confined to either the systemic or mucosal immune compartments.}

The degree to which immune priming in one anatomic compartment provides antigenexperienced cells and antibody at another site depends mostly on the immunogenicity and the amount of the priming antigen (Blanas et al., 2000), and the degree of inflammatory response induced (Lefrancois et al., 1999); factors known to influence the overall magnitude of the 
adaptive immune response. Another factor is whether the antigen or invading organism remains confined to the initial priming site. London et al. (1987) demonstrated a higher reovirus specific pCTL frequency in the Peyer's patches and mesenteric lymph nodes compared to the spleen and peripheral lymph nodes following per-oral infection of mice with reovirus T1L, and assumed these differences in frequency of pCTL in the peripheral lymph nodes and spleen were due to the dissemination of enterically primed pCTL, not cells primed locally in the systemic periphery by disseminating antigen or replicating virus, either freely or in association with migrating APC (Dharakul et al., 1988; Brown and Offit, 1998). Infection of the intestinal mucosa of immunocompetent mice with LCMV (Muller et al., 2000), rotavirus (Uhnoo et al., 1990), or reovirus (Letvin et al., 1981; Major and Cuff, 1997), can result in the dissemination of replicating virus to the mesenteric lymph nodes and spleen. Parenteral infection of mice with reovirus results in the recovery of replicating virus from the small intestinal crypts (Rubin et al., 1985) as well as the Peyer's patches, peripheral lymph nodes, and spleen (Letvin et al., 1981), quite possibly in association with migrating dendritic cells and macrophages. Indeed, macrophages and dendritic cells have been observed to migrate into the Peyer's patches from the non-mucosal periphery (Soesatyo et al., 1993). Dendritic cells containing LCMV mRNA have been observed by in situ hybridization in the small intestine lamina propria and intestinal epithelium of parenterally infected mice (Muller et al., 2000), suggesting dissemination of LCMV to the mucosa from the systemic periphery. Infectious virions as well as non-replicating endocytosed reovirus antigen may gain access to the systemic periphery from the mucosa or the reverse within migrating antigen presenting cells, as has been suggested in studies of rotavirus infection (Dharakul et al., 1988; Brown and Offit, 1998). Furthermore, dissemination of antigen in association with dendritic cells from the intestinal mucosa has been observed. Cells recovered 
from the thoracic duct lymph of mesenteric lymphadenectomized mice include numerous dendritic cells, termed 'veil cells' due to cell membrane folds that resemble veils (Liu and Macpherson, 1991; Liu and Macpherson, 1993). These dendritic cells are presumed to originate in the lamina propria and Peyer's patches, and contained experimentally fed ovalbumin protein. Furthermore, adoptive transfer of these cells elicited ovalbumin-specific $\mathrm{T}$ cell responses in recipient mice, indicating that cells migrating from the intestine are able to process and present antigen gathered in the intestine to naïve $\mathrm{T}$ cells in systemic tissues. In any event, regardless of the magnitude of free or dendritic cell-associated antigen dissemination, replicating virus can be isolated from the spleen following oral infection with reovirus, suggesting at least some of the pCTL in the non-mucosal systemic periphery may be primed in situ.

\section{Differences in the systemic and mucosal humoral immune responses may reflect underlying $T$ helper $1 / T$ helper 2 or other microenvironmental differences.}

As mentioned, there are distinct features of the systemic and mucosal immune responses. The mucosal humoral response is predominately of the IgA isotype, whereas that of the systemic periphery is mainly IgG. In order to determine if the IgA response was a byproduct of a chronic state of immune activation in Peyer's patches mediated by intestinal microflora, or an intrinsic feature of the MALT microenvironment, Weinstein and Cebra, (1991) infected germ-free mice either orally or parenterally with reovirus T1L. Germinal center reactions caused by proliferating B cells, otherwise absent in germ-free animals, were induced by reovirus infection. Immunohistochemical analysis of germinal center B cells showed that the reovirus infection induced surface $\operatorname{IgG}^{+}\left(\mathrm{sIgG}^{+}\right)$and $\operatorname{sIgM}^{+} \mathrm{B}$ cells in the draining lymph nodes, and a major $\operatorname{sIg} \mathrm{A}^{+}$ B cell response with a minor population of $\operatorname{sgM}^{+} \mathrm{B}$ cells in the Peyer's patches. Restriction enzyme digestion of the germinal center B cell DNA and subsequent southern blot analysis of the fragments confirmed rearrangement of the immunoglobulin heavy chain isotype genes. 
Similar isotypic responses were found using germ free mice mono-associated with Morganella enterically or parenterally injected, and are in keeping with isotype responses elicited from conventionally reared animals (Logan et al., 1991). Thus the preferential IgA response of the Peyer's patches is due to some intrinsic factor of the Peyer's patches not active in the peripheral lymphoid tissues. IgA isotype switching is known to be induced by T helper 2 type cytokine TGF- $\square$ Sonoda et al., 1992; van Vlasselaer et al., 1992), in addition to the interaction of B-cell expressed CD40 with T helper cell CD40L. IL-5, a T helper 2-type cytokine, is a known inducer of IgA production (Cebra et al., 1991a; Cebra et al., 1991b; Sonoda et al., 1992). IL-6 is yet another T helper 2 cytokine which augments IgA responses (Cebra et al., 1991b; McGhee et al., 1991). Therefore in mucosal immune responses, such as the response to enteric reovirus infection generated in the Peyer's patches (VanCott et al., 2000), a T helper 2 cell response might be expected to predominate and underlie the observed $\operatorname{sig} \mathrm{A}^{+} \mathrm{B}$ cell response (Cebra et al., 1991b).

Enteric reovirus infection induces predominantly a T helper 1 type cytokine profile, however, with upregulation of IFN- $\gamma$ message and production of IFN- $\gamma$ and IL-2 proteins (Fan et al., 1998). Furthermore, the immunoglobulin of the minor IgG isotype fraction induced by enteric reovirus infection is of the IgG2a and IgG2b subclasses (Major and Cuff, 1996), which are associated with IFN- $\gamma$ production by T helper 1 cells (Bossie and Vitetta, 1991). By contrast, the IgG antibody produced following parenteral infection with reovirus has a substantial IgG1 component in addition to IgG2a (Major and Cuff, 1996), indicating a potentially mixed underlying T helper 2/T helper 1 cytokine response. Nevertheless, semi-quantitative RT-PCR assessment of $\mathrm{T}$ helper 2/T helper 1 cytokine mRNA message levels in peripheral lymph nodes and Peyer's patches following parenteral and oral infection, respectively, were not found to be 
different (Major and Cuff, 1996). Thus, the use of antibody as a teleological assessment of the differences in T cell populations primed in Peyer's patches and peripheral lymph nodes is not sustained at the level of the $\mathrm{T}$ helper cell cytokine response with regard to classic $\mathrm{T}$ helper 2/T helper 1 cytokines such as IL-4, IL-5, IL-6 and IFN- $\gamma$, although the role of other cytokines, such as IL-10, IL-12, IL-15, and IL-18 remains to be determined. Nevertheless, assessment of T cell immune responses to reovirus in other mucosal tissues may to clarify the issue. Mixed Thelper 2/T helper 1 cytokine production is observed using intracellular cytokine staining and flow cytometry of pulmonary airway infiltrating $\mathrm{CD} 4^{+}$lymphocytes during reovirus bronchopneumonia (Thompson et al., 1999). T cell mediated immune responses generated within the priming sites of the lung, the tracheobronchial and mediastinal lymph nodes, are preferentially of the T helper 2 type (Constant et al., 2000), and it is similarly thought that most intestinal immune responses stimulated within the Peyer's patches also follow a default $\mathrm{T}$ helper 2 program, except in cases where a sufficient inflammatory cytokine override accompanies the antigen (Strober and Kelsall, 1998; Stumbles et al., 1998). Inflammation acts at the level of the antigen presenting cells, dendritic cells and macrophages, priming the naïve antigen specific responder $\mathrm{T}$ cells. The adoption of a $\mathrm{T}$ helper 1 immune response is governed in large part by dendritic cell and macrophage produced IL-12 (Fuss et al., 1999) and IL-15 (Liu et al., 2000) in response to local levels of inflammatory mediators and cytokines such as IL-16 (Mathy et al., 2000) and IL-18 (Liete-de-Morales et al., 1999; Chang et al., 2000). Thus the confusing array of $\mathrm{T}$ cell mediated and influenced responses share a common initiator focus, the priming APC, and it is from the event of the priming of the naïve virus-specific $\mathrm{T}$ cell response that all subsequent tissue dependent priming differences will arise. 


\section{Dendritic cells at different anatomic locations are functionally distinct as regards $T$ cell priming and differentiation, as evidenced by $T$ helper cell cytokine profiles.}

Evidence exists that the dendritic cell populations of the MALT are functionally different from those of the spleen or peripheral lymph nodes. Murine intestinal lamina propria dendritic cells pulsed with keyhole limpet haemocyanin preferentially stimulated naïve $\mathrm{CD}^{+} \mathrm{T}$ cell proliferation in vitro (Williams et al., 1992). By contrast, splenic and peripheral lymph node dendritic cells preferentially stimulated $\mathrm{CD}^{+} \mathrm{T}$ cells in vitro despite comparable surface expression of MHC class I and class II molecules (Williams et al., 1992). Further, upon monoclonal antibody ligation of CD40, murine dendritic cells isolated from Peyer's patches, but not those from the spleen, were shown to produce IL-10 (Iwasaki and Kelsall, 1999), a pleiotropic cytokine that induces $\mathrm{CD}^{+} \mathrm{T}$ cell proliferation and effector function (Rowbottom et al., 1999; Santin et al., 2000), B cell proliferation (Itoh and Hirohata, 1995), and Th2 responses (Cua et al., 1996). Dendritic cells also induce differential T helper 2/T helper 1 cytokine profiles by mechanisms as yet incompletely understood (Schuhbauer et al., 2000). For example, in vitro stimulation of naive transgenic $\mathrm{CD}^{+} \mathrm{T}$ cells bearing a TCR specific for an epitope from ovalbumin with Peyer's patch dendritic cells pulsed with ovalbumin induced the production of T helper 2 type cytokines, whereas splenic dendritic cells induced a mixed T helper 2/T helper 1 cytokine response (Iwasaki and Kelsall, 1999). Everson et al., (1998) showed mitogen and allogenic cell-stimulated Peyer's patch and splenic T cells produce T helper 2 type cytokines IL4 and IL-6 protein in the presence of Peyer's patch derived dendritic cells. By contrast, the presence of splenic DC tended to induce IL-2 and IFN- $\gamma$ among similarly stimulated T cells, and little IL-4 or IL-6, again suggesting an underlying difference in intestinal and systemic dendritic cells. Dendritic cells from other mucosal tissues aside from the intestine also induce $\mathrm{T}$ cell function distinct from the dendritic cells of non-mucosal lymphoid tissues. For instance, rat 
bronchus associated lymphoid tissue (BALT) dendritic cells pulsed in vitro with ovalbumin and subsequently intravenously transferred into recipient rats previously adoptively transferred with transgenic $\mathrm{CD} 4{ }^{+} \mathrm{T}$ cells bearing a TCR specific for an ovalbumin derived epitope induced an ovalbumin-specific T helper 2 response in naïve recipient rats (Stumbles et al., 1998). A mixed $\mathrm{T}$ helper 2/T helper 1 response could be obtained if BALT dendritic cells were incubated with the cytokine GM-CSF prior to ovalbumin pulsing, and a dominant T helper 1 response could be obtained if TNF- $\gamma$ was added in addition to GM-CSF, possibly by mimicking a cytokine milieu reminiscent of an inflammatory response (Stumbles et al., 1998). Another difference with regard to intestinal immune tissue is the anatomic fact that while peripheral lymph nodes receive activated dendritic cells and antigen via the afferent lymphatics, Peyer's patches have no afferent lymphatics (Witmer and Steinman., 1998). However, Peyer's patch dendritic cells in the subepithelial dome region underlying the $\mathrm{M}$ cells showed the ability to migrate in vitro in response to the inflammatory chemokine MIP-3 $\alpha$, whereas splenic dendritic cells lacked the ability to migrate in response to MIP-3a (Iwasaki and Kelsall, 2000). Furthermore, parenteral injection of soluble Toxoplasma gondii tachyzoite protein antigen caused dendritic cells of the subepithelial dome region off Peyer's patches to migrate deep into the $\mathrm{T}$ cell rich paracortical regions of the patches (Iwasaki and Kelsall, 2000). This migration in response to inflammation may be analogous to the migration of peripheral dendritic cells to the draining peripheral lymph nodes via the afferent lymphatics or via the blood to the spleen. Clearly then, the premier initiator APC of adaptive immunity has distinct functional characteristics in the systemic periphery and mucosal immune compartments (Iwasaki and Kelsall, 2000). 


\section{Dendritic cells are also capable of unconventional epitope processing and presentation.}

The effect of dendritic cells from mucosal or systemic peripheral immune tissue on the priming of $\mathrm{CD}^{+} \mathrm{T}$ cells is not well understood. Long believed to be primed exclusively by MHC class I molecules in association with cytosol derived cognate antigen, $\mathrm{CD} 8^{+}$cells can also be primed by endocytosed exogenous antigen in a manner normally associated with the MHC class II presentation pathway by an alternative mechanism phenomenon called 'cross-presentation' (Castellino et al., 2000; Hamano et al., 2000). Furthermore, CD8 ${ }^{+}$CTL responses have long been thought to be dependent on concomitant Th1 responses due to the direct relationship between and IL-18 (Okamoto et al., 1999), IL-12 (Paganin et al., 1995), IFN- $\gamma$ and IL-2 (Mehorotra et al., 1995) and the magnitude of the CTL response. Recently, CTL responses have been shown to be enhanced by $\mathrm{Th} 2$ type cytokines. For example, $\mathrm{CD} 8^{+} \mathrm{T}$ cells can proliferate in response to exogenous IL-10 (Rowbottom et al., 1999), and both IL-10 (Santin et al., 2000) and IL-4 (Specht et al., 1998) have been shown to enhance $\mathrm{CD} 8^{+} \mathrm{T}$ cell cytotoxicity. As CTL responses can be induced in the absence of Th cell assistance provided sufficient levels of cognate antigen presentation, co-stimulatory molecule expression, and cytokine production by priming dendritic cells, it is clear that the priming dendritic cells population may most heavily influence primary CTL responses. As conventional naïve T cells show no differences in recruitment to mucosal and non-mucosal priming sites, which recruit equally all $\mathrm{CD} 62 \mathrm{~L}^{+}$naïve $\mathrm{T}$ cells any differences in the CTL populations primed in the mucosa or peripheral lymph nodes would likely be due to local dendritic cells influence. 


\section{Dendritic cells at different anatomic locations may be functionally distinct as regards epitope processing and presentation; a mechanism of possible tissue-specific $T$ cell priming as a function of microenvironment.}

As previously mentioned, antigen-specific $\mathrm{CD}^{+}$and $\mathrm{CD} 4^{+} \mathrm{T}$ cells utilize their TCR to recognize cognate epitopes in association with MHC class I or class II, respectively. The generation of a panel of small peptide fragment epitopes from native foreign protein is dependent on the antigen processing and presentation pathways of the APC. It is unknown if dendritic cells from MALT or priming sites of the systemic periphery generate differential antigenic epitopes from the same native protein. However, it is known that dendritic cells can process and display differential epitopes of a given protein in response to exogenous cytokine tension. For instance, splenic dendritic cells pulsed with myelin basic protein process and display a characteristic panel of MHC class II associated epitopes, but treatment with exogenous IL-6 causes the dendritic cells to express novel epitopes, known as cryptic epitopes because they are otherwise not processed or expressed (Drakesmith et al., 1998). The IL-6 is believed to cause a decrease in the $\mathrm{pH}$ of dendritic cells endolysosomal vesicles, enhancing the activity of endogenous acid proteases to liberate the cryptic epitopes. It is as yet unknown whether other cytokines or chemokines of the local tissue milieu can similarly affect the panel of epitopes generated, although the ability of and type 2 interferons to enhance the function of the cytosolic multicatalytic proteosome and to enhance MHC class I expression of cryptic epitopes has been described (Sewell et al., 1999). Given the ability of dendritic cells to cross-present endocytosed antigen epitopes on MHC class I molecules, it is possible the IL-6 mediated alteration of antigen processing can also affect the priming of CTL populations in vivo (Drakesmith et al., 1998). 


\section{Tissue-specific modification of an extant, ongoing CTL response may reshape the final TCR repertoire.}

Immune responses are dynamic, as opposed to static events. Activated $\mathrm{CD} 8^{+} \mathrm{T}$ cells can migrate to peripheral sites of inflammation, assume effector function, and either die by in situ apoptosis or migrate via the lymphatics to draining lymph nodes. Within the lymph node, the antigen-experienced T cell will encounter APC which will express antigen acquired from the site of inflammation. The recently arrived effector CTL will recognize cognate epitopes on the APC by MHC-peptide-TCR interaction, possibly resulting in activation induced cell death (AICD) (Sarin et al., 1995). Ironically, CTL recognizing subdominant epitopes, or bearing TCR with less avidity for cognate antigen and thus less prone to being strongly activated by cognate epitopes presented by the APC, may more likely be spared than those CTL recognizing dominant antigens or having high avidity TCR (She et al., 1998). Extreme examples of this tendency toward AICD, termed 'clonal exhaustion', are seen in the deletion of the most avid CTL in chronic LCMV infection of mice and hepatitis B virus infection of humans, and are due to chronic stimulation of effector CTL by viral antigen (Welsh and McNally, 1999). However, in view of the predisposition for IL-10 production by the dendritic cells of the mucosa, a cytokine known to decrease epitope presentation (Faulkner et al., 2000), and also the production of immunosuppressive prostaglandin $\mathrm{E}_{2}$ (Newberry et al., 1999) and TGF- $\beta$ by macrophages and dendritic cells of the mucosa, effector CTL may receive less re-stimulation with in the mucosal lymphoid tissue compared to draining peripheral lymph nodes. Thus, mucosal CTL may be more likely to survive encounters with APC within the mucosa by avoiding AICD as compared to effectors in the systemic periphery. Additionally, CTL primed later in the immune response, after the generation of the $\mathrm{T}$ helper cell response, may respond to epitopes distinct from those primed in the very beginning, as $\mathrm{T}$ helper cell cytokine and CD40L mediated 'conditioning' of dendritic 
cells (Andreason et al., 2000) could allow for the generation and expression of cryptic epitopes. Indeed, the IL-6 dependent unmasking of cryptic epitopes subsequent to the priming of cryptic antigen specific $\mathrm{T}$ cell populations is thought to be most pronounced during an ongoing, previously established T helper 2 response (Drakesmith et al., 1998).

\section{Evidence for continuous reshaping of the $T$ cell repertoire in the intestinal mucosa.}

Arstila et al., (2000) demonstrated that activated 'blasting' (proliferating) $\mathrm{CD}^{+}$and $\mathrm{CD} 8^{+}$cells trafficking through the thoracic duct circulated back to the intestinal mucosal. Indeed, many of the activated T cells had TCR $\beta$ chains identical to TCR of cells distributed through the oligoclonal populations of the small intestinal lamina propria and IEL populations. Thus, $\mathrm{CD} 8^{+}$ cells restimulated within the effector site become activated and induced to proliferate, and recirculate through the intestinal lymphatics to the peripheral circulation and back to the intestinal mucosa. This observation, taken with the characteristic oligoclonality of conventional $\mathrm{CD}^{+} \mathrm{T}$ cells in the IEL and lamina propria (Regnault et al., 1995; Arstila et al., 2000) suggests a continuous modification of the antigen specific $\mathrm{T}$ cell repertoire occurs well after the initial antigen priming event in the Peyer's patches. Modification of the CTL repertoire in enteric reovirus infection may thus be more pronounced in the case of intestine than in the systemic periphery. This is possibly due to the fact that intestinal epithelial cells (IEC) are believed to act as non-classical APC, as they express MHC class I and can be induced to express class II in response to TNF- $\alpha$ and IFN- $\gamma($ Lopes et al., 1999; Hershberg and Mayer, 2000). IEC also express novel co-stimulatory molecules such as gp180 (Hershberg and Mayer, 2000), which interacts with $\mathrm{T}$ cell surface molecule CD2 (Horie et al., 1998) to induce T cell activation by an

alternative mechanism as yet not entirely understood (Bachmann et al., 1999). IEC, like resident professional APC such as tissue macrophages and dendritic cells, may be exposed to antigen 
epitopes unseen in the Peyer's patches as the initial phase of infection requires chymotrypic processing of the outer capsid proteins $\sigma 3$ and $\sigma 1 \mathrm{c}$ to yield the M cell-binding ISVP (Bodkin $e t$ al., 1998). Cleaved outer capsid proteins taken up directly by IEC or transported to the lamina propria by transcellular or paracellular routes may be later presented to reovirus-specific CTL. It has been established that serum and tissue protease-mediated extracellular proteolytic processing of viral antigens, such as the $\delta$ antigen of the hepatitis D virus, can result in the priming of T cell responses tissue macrophage (Accapezzato et al., 2000). Additionally, specialized dendritic cells subpopulations resident in the intestinal mucosal have been identified that phagocytose apoptotic IEC and subsequently migrate via the villus-draining lacteal to the mesenteric lymph nodes and via the thoracic duct to the spleen. It is possible that such dendritic cells may cross-present antigenic epitopes generated in the intestinal lumen or in the mucosa that are unseen in the systemic periphery (Huang et al., 2000). Such dendritic cells would influence both the initial priming of the responder T cells and could modify the repertoire of antigen experienced T cells (Albert et al., 2000).

\section{Differential reshaping of the virus-specific CTL repertoire as a function of distinct humoral immunity.}

Cross-presentation pathways are facilitated by the binding Fc receptors on dendritic cells to antibody complexed with foreign antigen (Regnault et al., 1999). Thus, immune complexes of antibody-antigen aggregates may interact with dendritic cells to modify the MHC class I restricted CTL response (Hamano et al., 2000). This could be important to the modification of CTL responses primed in the mucosal or systemic periphery given that evidence exists that antibody induced at mucosal sites may recognize some conformational antigenic determinants or epitopes distinct from those recognized by systemically primed antibody responses, due possibly to the local antigenic milieu (Bos et al., 1988). Modification of the CTL response by these 
mechanisms is, of course, speculative, as no evidence exists to support or refute the influence of the route of infection on the populations of responder CTL. Determination of differences in induced CTL populations would be a teleological assessment of distinct antigen processing and MHC class I presentation in much the same way as the $\mathrm{T}$ helper 2/T helper 1 cytokine profiles reflect MHC class II mediated T cell priming. Elucidating the mechanisms of antigen specific T cell priming, the central event of adaptive immunity, in general and as pertains to mucosal immunity, is of paramount importance for such applications as vaccine design, tolerance induction, and the control of immunopathology.

\section{Overview of these studies}

Oral and parenteral reovirus infections induce both virus-specific CTL and antibody. However, the humoral immune response to oral reovirus infection is dominated by antibody of the $\operatorname{IgA}$ isotype, whereas infection of the systemic periphery is dominated by $\operatorname{IgG}$ (Weinstein and Cebra, 1991). Furthermore, such IgG as is induced following intestinal reovirus infection is predominantly of a different subclass than that induced following parenteral infection (Major and Cuff, 1996). It has been suggested that these differences are due to intrinsic differences in the lymphocyte priming conditions of the intestinal and peripheral immune tissue environments, and may reflect differences in the $\mathrm{T}$ cell populations induced in these tissues. Indeed, evidence exists for the induction of unusual $\mathrm{T}$ cell populations during reovirus infection of the pulmonary mucosal (Thompson et al., 1996; Periwal and Cebra, 1999). These studies were undertaken primarily to determine what differences, if any, exist in the CTL populations that are induced following oral or parenteral infection with reovirus. Furthermore, other aims included determining if different immune compartments of the intestine contained distinct CTL populations following oral infection. Additionally, we sought to determine the effects of ageing 
on CTL induction, humoral immunity, and immune memory following oral and parenteral reovirus infection.

\section{The CTL V $\beta$ Repertoire Induced Following Oral or Systemic Infection with Reovirus.}

We hypothesized that virus-specific CTL populations induced following oral reovirus T1L infection would be distinct from those induced following parenteral infection. We assessed $\mathrm{CD}^{+}$cells from the Peyer's patches and spleens of orally infected mice and popliteal lymph nodes and spleens of mice infected in the hind footpads for changes in the V $\beta$ repertoire by flow cytometry. We also established cell lines from these tissues to enrich for virus-specific CTL, and concomitantly assessed for changes in the $\mathrm{V} \beta$ repertoire of $\mathrm{CD}^{+} \mathrm{T}$ cells. We also adoptively transferred $\mathrm{CD}^{+}$cells from the spleens and Peyer's patches of orally infected donor mice, and the spleens of hind footpad infected mice, into reovirus infected SCID recipient mice, and assessed for changes in the $\mathrm{V} \beta$ repertoire of recovered $\mathrm{CD} 8^{+} \mathrm{T}$ cells. We observed a consistent expansion of virus specific $\mathrm{V} \beta 6^{+} \mathrm{CD} 8^{+} \mathrm{CTL}$ for both orally infected and hind footpad infected mice. Subsequently, we assessed the $\mathrm{V} \beta 6^{+} \mathrm{CD} 8^{+} \mathrm{T}$ cells, which dominate the reovirus-specific CTL response, for differences in the CDR3 $\beta$ length profiles of cells derived from orally infected and footpad infected donors, to better determine if distinct clonal populations of the general $\mathrm{V} 6^{+} \mathrm{CD}^{+} \mathrm{T}$ cell response were induced following oral or parenteral reovirus infection. We observed a the expansion of a clonal population(s) of $\mathrm{V} 6^{+} \mathrm{CD}^{+} \mathrm{T}$ cells utilizing the same length CDR3 $\beta$ region, regardless of the route of infection. We conclude that the CTL population induced following oral reovirus infection is similar, if not identical to the CTL population induced following parenteral reovirus infection. 


\section{Preservation of Intestinal Immunity to Reovirus Infection in Ageing.}

It has been reported that intestinal immunity is diminished in ageing (Schmucker et al., 1996; Koga et al., 2000). We hypothesized that the intestinal CTL and humoral immune response to reovirus infection would be diminished in aged mice compared to young mice. We assessed the splenic pCTL, and the virus-specific cytotoxicity of splenic and Peyer's patch cells from aged and young mice infected orally with reovirus. We also assessed the intestinal IgA response and systemic IgG response to oral reovirus infection in aged and young mice. We observed similar CTL frequencies and levels of virus-specific cytotoxicity for old and young mice, and similar systemic IgG responses to oral reovirus infection. However, intestinal IgA responses were elevated in old mice compared to young mice, suggesting maintenance of intestinal CTL responsiveness and possible potentiation of intestinal humoral immunity in ageing. 


\section{References}

Accapezzato, D., Nisini, R., Paroli, M., Bruno, G., Bonino, F., Houhgton, M., and V. Barnaba. 1998. Generation of an MHC class II-restricted T cell epitope by extracellular processing of hepatitis delta antigen. J. Immunol. 160:5262-5256.

Akolkar, P. N., Gulwani-Akolkar, B., McKinley, M., Fisher, S.E., and J. Silver. 1995.

Comparisons of $\mathrm{T}$ cell receptor (TCR) V $\beta$ repertoires of lamina propria and peripheral blood lymphocytes with respect to frequency and oligoclonality. Clin. Immunol. Immunopathol. 76:155-163.

Albert, M. L., Sauter, B., and N. Bhardwaj. 1998. Dendritic cells acquire antigen from apoptotic cells and induce class I-restricted CTL. Nature. 392:86-89.

Alexander-Miller, M. A., Leggat, G.M., Sarin, A., and J.A. Berzofsky. 1996. Role of antigen, CD8, and cytotoxic T lymphocyte (CTL) avidity in high dose antigen induction of apoptosis of effector CTL. J. Exp. Med. 184:485-492.

Andreasen, S. O., Christensen, J. E., Marker, O., and A.R. Thomsen. 2000. Role of CD40 ligand and CD28 in induction and maintenance of antiviral CD8+ effector T cell responses. J. Immunol. 164:3689-3697. 
Angel, J., Franco, M. A., Greenberg, H. B., and D. Bass. 1999. Lack of a role for type I and type II interferons in the resolution of rotavirus-induced diarrhea and infection in mice. J. Inter. Cyto. Res. 19:655-659.

Anjuere, F., Martin, P., Ferrero, I., Fraga, M.L., del Hoyo, G.M., Wright, N., and C. Ardavin. 1999. Definition of dendritic cell subpopulations present in the spleen, Peyer's patches, lymph nodes, and skin of the mouse. Blood. 93:590-598.

Arena, A., Liberto, M. C., Iannello, D., Capozza, A. B., and A. Foca. 1999. Altered cytokine production after human herpes virus type 6 infection. New Microbiol. 22:293-300.

Arenz, M., Herzog-Hauff, S., Meyer zum Buschenfelde, K. H., and H. F. Lohr. 1997. Antigenindependent in vitro expansion of $\mathrm{T}$ cells does not affect the $\mathrm{T}$ cell receptor $\mathrm{V}$ beta repertoire. $\mathrm{J}$. Mol. Med. 75:678-686.

Arstila, T., Arstila, T. P., Calbo, S., Selz, F., Malassis-Seris, M., Vassalli, P., Kourilsky, P., and D. guy-Grand. 2000. Identical T cell clones are located in the mouse gut epithelium and lamina propria and circulate in the thoracic duct lymph. J. Exp. Med. 191:823-834.

Banchereau, J., Briere, F., Caux, J., Davoust, J., Lebecque, S., Liu, Y.J., Pulendron, B., and K. Palucka. 2000. Immunobiology of dendritic cells. Annu. Rev. Immunol. 18:767-811. 
Bachmann, M.F., Schorle, H., Kuhn, R., Muller, W., Hengartner, H., Zinkernagel, R.M., and I. Horak. Antiviral immune responses in mice deficient for both interleukin-2 and interleukin-4. J. Virol. 69:4842-4846.

Bachmann, M. F., Barner, M., and M. Kopf. 1999. CD2 sets quantitative thresholds in T cell activation. J. Exp. Med. 190:1383-1391.

Badovinac, V. P., and J. T. Harty. 2000. Intracellular staining for TNF and IFN-gamma detects different frequencies of antigen-specific CD8(+) T cells. J. Immunol. Methods. 238:107-117. Barton, E.S., Forrest, J.C., Connolly, J.L., Chappell, J.D., Liu, Y., Schnell, F.J., Nusrat, A., Parkos, C.A., and T.S. Dermody. 2001. Junction adhesion molecule is a receptor for reovirus. Cell. 104:441-451.

Bennet, J. D., Brown, W. R., and B. L. Kotzin. 1999. Regional variation in the lamina propria T cell receptor V beta repertoire in normal human colon. Clin. Immunol. 90:38-46.

Benninger-Doring, G., Pepperl, S., Deml, L., Modrow, S., Wolf, H., and W. Jilg. 1999. Frequency of CD8(+) T lymphocytes specific for lytic and late antigens of Epstein-Barr virus in healthy virus carriers. Virology. 264:289-297.

Berin, M.C., Yang, P.C., Ciok, L., Waserman, S., and M.H. Purdue. 1999. Role for IL-4 in macromolecular transport across human intestinal epithelium. Am. J. Physiol. 276:1046-1052. 
Berquist, C., Lagergard, T., Lindblad, M., and J. Holmgren. 1995. Local and systemic antibody responses to dextran-cholera toxin B subunits. Infect. Immun. 63:2021-2025.

Blanas, E., Davey, G. M., Carbone, F. R., and W. R. Heath. 2000. A bone marrow-derived APC in the gut-associated lymphoid tissue captures oral antigens and presents them to both CD4+ and CD8+ T cells. J. Immunol. 164:2890-2896.

Bloom, E. T., and J. A. Horvath. 1994. Cellular and molecular mechanisms of the IL_12-induced increase in allospecific murine cytotoxic $\mathrm{T}$ cell activity. Implications for the age related decline in CTL. J. Immunol. 152:4242-4254.

Biron, C. A. 1999. Initial and innate responses to viral infections - pattern setting in immunity or disease. Curr. Opin. Microbiol. 2:374-381.

Bode, U., Duda, C., Weidner, F., Rodriguez-Palmero, M., Wonigeit, K., Pabst, R., and J. Westermann. 1999. Activated T cells enter rat lymph nodes and Peyer's patches via high endothelial venules: survival by tissue-specific proliferation and preferential exit of CD8+ T cell progeny. Eur. J. Immunol. 29:1487-1495.

Bodkin, D. K., Nibert, M. L., and B. N. Fields. 1989. Proteolytic digestion of reovirus in the intestinal lumens of neonatal mice. J. Virol. 63:4676-4681. 
Bos N.A., Meeuwsen, C.G., Wostmann, B.S., Pleasants, J.R., R. Brenner. 1988. The influence of exogenous antigenic stimulation on the specificity repertoire of background immunoglobulinsecreting cells of different isotypes. Cell. Immunol. 112:371-380.

Bossie, A., and E. S. Vitetta. 1991. IFN-gamma enhances secretion of IgG2a from IgG2acommited LPS-stimulated murine B cells: implications for the role of IFN-gamma in class switching. Cell. Immunol. 135:95-104.

Bousso, P., Lemaitre, F., Laouini, D., Kanellopoulos, J., and P. Kourilsky. The peripheral CD8 T cell repertoire is largely independent of the presence of intestinal flora. Int. Immunol. 12:425430.

Bousso, P., Levraud, J. P., Kourilsky, P., and J. P. Abastado. 1999. The composition of a primary $\mathrm{T}$ cell response is largely determined by the timing of recruitment of individual $\mathrm{T}$ cell clones. $J$. Exp. Med. 189: 1591-1600.

Brennan, F.R., Bellaby, T., Helliwell, S.M., Jones, T.D., Kamstrup, S., Dalsgaard, K., Flock, J.I., W.D. Hamilton. 1999. Chimeric plant virus particles administered nasally or orally induce systemic and mucosal immune response in mice. J. Virol. 73:930-938.

Busch, D. H., and E. G. Pamer. 1998. MHC class I/peptide stability: implications for immunodominance, in vitro proliferation, and diversity of responding CTL. J. Immunol. 160: $4441-4448$. 
Busch, D. H., Pilip, I., and E.G. Pamer. 1998. Evolution of a complex T cell receptor repertoire during primary and recall bacterial infection. J. Exp. Med. 188:61-70.

Busch, D. H., and E.G. Pamer. 1999. T cell affinity maturation by selective expansion during infection. J. Exp. Med. 189:701-710.

Butz, E. A., and M. J. Bevan. 1998. Massive expansion of antigen-specific CD8+ T cells during an acute virus infection. Immunity. 8:167-175.

Callan, M. F., Annels, N., Steven, N., Tan, L., Wilson, J., McMichael, A.J., and A.B. Rickson. 1998. T cell selection during the evolution of CD8+ T cell memory in vivo. Eur. J. Immunol. 28:4382-4390.

Camerini, V., Sydora, B. C., Aranda, R., Nguyen, C., MacLean, C., McBride, W. H., and M. Kronenberg. 1998. Generation of intestinal mucosal lymphocytes in SCID mice reconstituted with mature, thymus-derived T cells. J. Immunol. 160:2608-2618.

Campos-Lima, P. O., Levitsky, V., Imreh, M. P., Gavioli, R., and M. G. Masucci. 1997. Epitopedependent selection of highly restricted or diverse $\mathrm{T}$ cell receptor repertoires in response to persistent infection by Epstein-Barr virus. J. Exp. Med. 186:83-89.

Cao, W., Myers-Powell, B. A., and T. J. Braciale. 1996. The weak CD8_CTL response to an influenza hemagglutinin epitope reflects limited T cell availability. J. Immunol. 157:505-511. 
Carbonari, M., Cibati, M., Pesce, A. M., Dell’Anna, L., D’Offizi, G., Angelici, A., Uccini, S., and M. Fiorilli. 1996. Comparison of the Vbeta repertoire in peripheral blood and in the lymph nodes of HIV-infected subjects reveals skewed usage predominantly in CD8+ T cells. Clin. Immunol. Immunopathol. 81:200-209.

Carman, P. S., Ernst, P. B., Rosenthal, K. L., Clark, D. A., Befus, A. D., and J. Bienenstock. 1986. Intraepithelial leukocytes contain a unique subpopulation of NK-like cytotoxic cells active in the defense of gut epithelium to enteric murine coronavirus. J. Immunol. 136:1548-1553.

Carson, W.E., Yu, H., Diersheide, J., Pfeffer, K., Bouchard, P., Clark, R., Durbin, J., Baldwin, A.S., Peschon, J., Johnson, P.R., Ku, G., Baumann, H., and M.A. Caliguri. 1999. A fatal cytokine-induced systemic cytokine response reveals a critical role for NK cells. J. Immunol. 162:4943-4951.

Castellino, F., Boucher, P. E., Eichelberg, K., Mayhew, M., Rothman, J.E., Houghton, A.N., and R.N. Germain. 2000. Receptor-mediated uptake of antigen/heat shock protein complexes results in major histocompatibility complex class I antigen presentation via two distinct processing pathways. J. Exp. Med. 191:1957-1964.

Castrucci, M. R., Donatelli, I., Sidoli, L., Barigazzi, G., Kawaoka, Y., and R.G. Webster. 1993. Genetic reassortment between avian and human influenza A viruses in Italian pigs. Virology. 193:503-506. 
Cebra, J. J., George, A., and R. L. Kerlin. 1991. A cautionary note concerning the possibility that transforming growth factor-beta is a switch factor that acts on primarily B cells to initiate IgA expression. Immunol. Res. 10:404-406.

Cebra, J. J., George, A., and C.E. Schrader. 1991. A microculture containing Th2 and dendritic cells supports the production of IgA by clones from both primary and IgA memory B cells and by single germinal center B cells from Peyer's patches. Immunol. Res. 10:389-392.

Cerwenka, A., Morgan, T. M., Harmsen, A. G., and R. W. Dutton. 1999. Migration kinetics and final destination of type 1 and type 2 CD8 effector cells predict protection against pulmonary virus infection. J. Exp. Med. 189:423-434.

Chang, J. T., Segal, B. M., Nakanishi, K., Okamura, H., and E. M. Shevach. 2000. The costimulatory effects of IL-18 on the induction of antigen-specific interferon- $\gamma$ production by resting T cells is IL-12 dependent and is mediated by upregulation of the IL-12 receptor $\beta 2$ subunit. Eur. J. Immunol. 30:1113-1119.

Chapman, H.A. 1998. Endosomal proteolysis and MHC class II function. Curr Opin. Immunol. 10:93-102.

Chardes, T., Velge-Roussel, F., Mevelec, P., Mevelec, M. N., Buzoni-Gatel, D., and D. Bout. 1993. Mucosal and systemic cellular immune responses induced by Toxoplasma gondii antigens in cyst orally infected mice. Immunology. 78:421-429. 
Chen, W., Yewdell, J. W., Levine, R. L., and J. R. Bennink. 1999. Modification of cysteine residues in vitro and in vivo affects the immunogenicity and antigenicity of major histocompatibility complex class I-restricted viral determinants. J. Exp. Med. 189:1757-1764.

Coffin, S. E., Klinek, M., and P. A. Offit. 1995. Induction of virus-specific antibody production by lamina propria lymphocytes following intramuscular inoculation with rotavirus. J. Infect. Dis. 172: 874-878.

Coffin, S. E., and P. A. Offit. 1998. Induction of mucosal B cell memory by intramuscular inoculation of mice with rotavirus. J. Virol. 72:3479-3483.

Coffin, S. E., Clark, S. L., Bos, N. A., Brubaker, J. O., and P. A. Offitt. 1999. Migration of antigen-presenting B cells from peripheral to mucosal lymphoid tissues may induce intestinal antigen-specific IgA following parenteral immunization. J. Immunol. 163:3064-3070.

Cole, G. A., Hogg, T. L., and D. L. Woodland. 1995. T cell recognition of the immunodominant Sendai virus NP324-332/Kb epitope is focused on the center of the peptide. J. Immunol. 155:2841-2848.

Constant, S. L., Lee, K. S., and K. Bottomly. 2000. Site of antigen delivery can influence T cell priming: pulmonary environment promotes preferential Th2-type differentiation. Eur. J. Immunol. 30:840-847. 
Corazza, N., Muller, S., Brunner, T, Kagi, D., and C. Mueller. 2000. Differential contribution of Fas- and perforin -mediated mechanisms to the cell-mediated cytotoxic activity of naïve and in vivo primed intestinal intraepithelial lymphocytes. J. Immunol. 164:398-403.

Couedel, C., Peyrat, M. A., Brossay, L., Koezuka, Y., Porcelli, S. A., Davodeau, F., and M. Bonneville. 1998. Diverse CD1d-restricted reactivity patterns of human T cells bearing “invariant” AV24BV11 TCR. Eur. J. Immunol. 28:4391-4397.

Couedel, C., Bodinier, M., Peyrat, M.A., Bonneville, M., Davodeau, F., and F. Lang. 1999. Selection and long-term persistence of reactive CTL clones during an EBV chronic response are determined by avidity, CD8 variable contribution compensating for differences in TCR affinities. J. Immunol. 162:6351-6358.

Couedel-Courteille, A., Le Grand, R., Tulliez, M., Guilllet, J.G., and A. Venet. 1997. Direct ex vivo simian immunodeficiency virus (SIV)-specific cytotoxic activity detected from the small intestine intraepithelial lymphocytes of SIV-infected macaques at an advanced stage of infection. J. Virol. 71:1052-1057.

Creery, W. D., Diaz-Mitoma, F., Filion, L., and A. Kumar. 1996. Differential modulation of B71 and B7-2 isoform expression on human monocytes by cytokines which influence the development of T helper cell phenotype. Eur. J. Immunol. 26:1273-1277. 
Croitoru, K., Ernst, P. B., Bienenstock, J., Padol, I.., and A. M. Stanisz. 1990. Selective modulation of the natural killer activity of murine intestinal intraepithelial leukocytes by the neuropeptide substance P. Immunology. 71:196-201.

Crowley, M. P., Fahrer, A. M., Baumgarth, N., Hampl, J., Gutgemann, I., Teyton, L., and Y. Chien. 2000. A population of murine gd T cells that recognize an inducible MHC class Ib molecule. Science. 287:314-316.

Cua, D. J., Coffman, R. L., and S. A. Stohlman. 1996. Exposure to Th2 cytokines in vivo before encounter with antigen selects for $\mathrm{T}$ helper subsets via alterations in antigen-presenting cell function. J. Immunol. 157:2830-2836.

Cuff, C. F., Cebra, C. K., Rubin, D. H., and J. J. Cebra. 1993. developmental relationship between cytotoxic alpha/beta $\mathrm{T}$ cell receptor-positive intraepithelial lymphocytes and Peyer's patch lymphocytes. Eur. J. Immunol. 23:1333-1339.

Cumberbatch, M., Dearman, R. J., and I. Kimber. 1996. Constitutive and inducible expression of Interleukin- 6 by Langerhans cells and lymph node dendritic cells. Immunology. 87:513-518.

Deeths, M. J., Kedl, R. M., and M. F. Mescher. 1999. CD8+ T cells become nonresponsive (anergic) following activation in the presence of costimulation. J. Immunol. 163:102-110. 
Denkers, E. Y., Caspar, P., and A. Sher. 1994. Toxoplasma gondii possesses a superantigen activity that selectively expands murine $\mathrm{T}$ cell receptor $\mathrm{V}$ beta5-bearing $\mathrm{CD} 8+$ lymphocytes. $J$. Exp. Med. 180:985-994.

Dharakul, T., Riepenhoff-Talty, M., Albini, B., and P. L. Ogra. 1988. Distribution of rotavirus antigen in intestinal lymphoid tissues: potential role in development of the mucosal immune response to rotavirus. Clin. Exp. Immunol. 74:14-19.

Doherty, P. C., Topham, D. J., Tripp, R. A., Cardin, R. D., Brooks, J. W., and P. G. Stevenson. 1997. Effector CD4+ and CD8+ T-cell mechanisms in the control of respiratory virus infections. Immunol. Rev. 159:105-117.

Donahoe, S. M., Moretto, W. J., Samuel, R. V., Marx, P. A., Hanke, T., Connor, R. I., and D. F. Nixon. 2000. Direct measurement of CD8+ T cell responses in macaques infected with simian immunodeficiency virus. Virology. 272:347-356.

Doyle, A. G., Buttigieg, K., Groives, P., Johnson, B. J., and A. Kelso. 1999. The activated type 1-polarized $\mathrm{CD} 8+\mathrm{T}$ cell population isolated from an effector site contains cells with flexible cytokine profiles. J. Exp. Med. 190:1081-1091.

Drakesmith, H., O’Neil, D., Schneider, S. C., Binks, M., Medd, P., Sercarz, E., Beverly, P., and B. Chain. 1998. In vivo priming of T cells against cryptic determinants by dendritic cells exposed to Interleukin 6 and native antigen. Proc. Natl. Acad. Sci. USA. 95:14903-14908. 
Dubois, P.M., Pihlgren, M., Tomkowiak, M., Van Mechelen, M., and J. Marvel. 1998. Tolerant CD8 T cells induced by multiple injections of peptide antigen show impaired TCR signaling and altered proliferative responses in vitro and in vivo. J. Immunol. 161:5260-5267.

Ehl, S., Hombach, J., Aichele, P., Hengartner, H., and R.M. Zinkernagel. 1997. Bystander activation of cytotoxic $\mathrm{T}$ cells: studies on the mechanism and evaluation of in vivo significance in a transgenic mouse model. J. Exp. Med. 185:1241-1251.

Ehl, S., Klenerman, P., Zinkernagel R. M., and G. Bocharov. 1998. The impact of variation in the number of CD8(+) T-cell precursors on the outcome of virus infection. Cell. Immunol. 189:67-73.

Everson, M.P., Lemak, D.G., McDuffie, D. S., Koopman, W. J., McGhee, J. R., and K.W. Beagley. 1998. Dendritic cells from Peyer's patch and spleen induce different T helper cell responses. J. Inter. Cyto. Res. 18:103-115.

Fan, J. Y., Boyce, C. S., and C. F. Cuff. 1998. T-helper 1 and T-helper 2 cytokine responses in gut-associated lymphoid tissue following enteric reovirus infection. Cell. Immunol. 188:55-63.

Farstad, I.N., Halstensen, T. S., Lien, B., Kilshaw, P. J., and A. I. Lazarovitz. 1996. distribution of b7 integrins in the human intestinal mucosal and organized gut-associated lymphoid tissue. Immunology. 89:227-237. 
Farstad, I. N., Halstensen, T. S., Kvale, D., Fausa, O., and P. Brandtzaeg. 1997. Topographic distribution of homing receptors on B and T cells in human gut-associated lymphoid tissue: relation of L-selectin and integrin alpha 4 beta 7 to naïve memory phenotypes. Am. J. Pathol. 150:187-199.

Faulkner, L., Buchan, G., and M. Baird. 2000. Interleukin-10 does not affect phagocytosis of particulate antigen by bone marrow-derived dendritic cells but does impair antigen presentation. Immunology. 99:523-531.

Feng, N., Burns, J. W., Bracy, L., and H. B. Greenberg. 1994. Comparison of mucosal and systemic humoral immune responses and subsequent protection in mice orally inoculated with a homologous or a heterologous rotavirus. J. Virol. 68:7766-7773.

Finberg, R., Weiner, H.L., Burakoff, S. J., and B. N. Fields. 1981. Type-specific reovirus antiserum blocks the cytotoxic T-cell-target cell interaction: evidence for the association of the viral hemagglutinin of a non-enveloped virus with the cell surface. Infect. Immun. 31:646-649.

Finch, P. W., and A. L. Cheng. 1999. Analysis of the cellular basis of keratinocyte overexpression in inflammatory bowel disease. Gut. 45:848-855.

Flynn, K. J., Riberdy, J. M., Christensen, J. P., Altman, J. D., and P. C. Doherty. 1999. In vivo proliferation of naïve and memory influenza-specific CD8(+) T cells. Proc. Natl. Acad. Sci. U.S.A. 96:8597-8602. 
Franco, M. A., Lefevre, P., Willems, P., Tosser, G., Lintermanns, P., and J. Cohen. 1994. Identification of cytotoxic T cell epitopes on the VP3 and VP6 rotavirus proteins. J. Gen. Virol. 75:589-596.

Friend, D. S., Gurish, M. F., Austen, K. F., Hunt, J., and R. L. Stevens. 2000. Senescent jejunal mast cells and eosinophils in the mouse preferentially translocate to the spleen and draining lymph node, respectively, during the recovery phase of helminth infection. J. Immunol. 165:344352.

Fujihashi, K., McGhee, J.R., Kweon, M.N., Cooper, M.D., Tonegawa, S., Takahashi, I., Hiroi, T., Mesteckey, J., and H. Kiyono. 1996. gamma-delta T-cell deficient mice have impaired immunoglobulin A responses. J. Exp. Med. 183:1929-1935.

Fuss, I. J., Marth, T., Neurath, M.F., Pearlstein, G.R., Jain, A., and W. Strober. 1999. Antiinterleukin 12 treatment regulates apoptosis of Th1 cells in experimental colitis in mice. Gastroenterology. 117:1078-1088.

Franco, M. A., and H. B. Greenberg. 1995. Role of B cells and cytotoxic T lymphocytes in clearance of and immunity to rotavirus infection in mice. J. Virol. 69:7800-7806.

Gajewski, T. F., Renauld, J. C., Van Pel, A., and T. Boon. 1995. Costimulation with B7-1, IL-6, and IL-12 is sufficient for primary generation of murine antitumor cytolytic T lymphocytes in vitro. J. Immunol. 154:5637-5648. 
Gallimore, A., Glithero, A., Godkin, A., Tissot, A. C., Pluckthun, A., Elliot, T., Hengartner, H., and R. Zinkernagel. 1998. Induction and exhaustion of lymphocytic choriomeningitis virusspecific cytotoxic $\mathrm{T}$ lymphocytes visualized using soluble tetrameric major histocompatibility complex class-I peptide complexes. J. Exp. Med. 187:1383-1393.

Gherardi, M. M., and M. Esteban. 1999. Mucosal and systemic immune responses induced after oral delivery of vaccinia virus recombinants. Vaccine. 17:1074-1083.

Gianfrani, C., Oseroff, C., Sideny, J., Chesnut, R. W., and A. Sette. 2000. Human memory CTL response specific for influenza A virus is broad and multispecific. Hum. Immunol. 61:438-452.

Goodman, T., and L. Lefrancois. 1988. Expression of the gamma-delta T cell receptor on intestinal CD8+ intraepithelial lymphocytes. Nature. 333:855-858.

Gray, C.M., Lawrence, J., Schapiro, J.M., Altman, J.D., Winters, M.A., Crompton, M., Loi, M., Kundu, S.K., Davis, M.M., and T.C. Merigan. 1999. Frequency of class I MHC-restricted antiHIV CD8+ T cells in individuals receiving highly active antiretroviral therapy (HAART). $J$. Immunol. 162:1780-1788.

Grayson, J. M., Zajac, A. J., Altman, J. D., and R. Ahmed. 2000. Cutting edge: increased expression of Bcl-2 in antigen-specific memory CD8+ T cells. J. Immunol. 164:3950-3954. 
Griffith, E., Ramsburg, E. and A. Hayday. 1998. Recognition of human gut gamma-delta cells of stress-inducible major histocompatibility molecules on enterocytes. Gut. 43:166-167.

Groh, V., Steinle, A., Bauer, S., and T. Spies. 1998. Recognition of stress-induced MHC molecules by intestinal epithelial gamma delta T cells. Science. 279:1737-1740.

Guimaraes, M. A., Nozawa, C.M., Guimaraes, A.C., and S. Ramos. 1997. Rotavirus and reovirus interaction with mouse peritoneal resident phagocytic cells. Braz. J. Med. Biol. Res. 30:11871190.

Guy-Grand, D., Malassis-Seris, M.., and P. Vassalli. 1991. Cytotoxic differentiation of mouse gut thymodependent and independent intraepithelial $\mathrm{T}$ lymphocytes is induced locally. Correlation between functional assays, presence of perforin and granzyme transcripts, and cytoplasmic granules. J. Exp. Med. 173:1549-52.

Guy-Grand, D., Cuenod-Jabri, B., Malassis-Seris, M., Selz, F., and P. Vassalli. 1996. Complexity of the mouse gut $\mathrm{T}$ cell immune system: identification of two distinct natural killer $\mathrm{T}$ cell intraepithelial lineages. Eur. J. Immunol. 26:2248-2256.

Haanen, J. B., Toebes, M., Cordaro, T.A., Wolkers, M. C., Kruisbeek, A.M., and T.N. Schumacher. 1999. Systemic T cell expansion during a localized viral infection. Eur. J. Immunol. 29:1168-1174. 
Hamano, Y., Arase, H., Saisho, H., and T. Saito. 2000. Immune complex and Fc receptormediated augmentation of antigen presentation for in vivo Th cell responses. J. Immunol. 164:6113-6119.

Hammond, S.A., Bollinger, R.C., Tobery, T.W., and R.F. Silliciano. 1993. Transporterindependent processing of HIV-1 envelope protein for recognition by CD8+ cells. Nature. 364:158-161.

Harty, J. T., Tvinnereim, A.R., and D. W. White. 2000. CD8+ T cell effector mechanisms in resistance to infection. Annu. Rev. Immunol. 18:275-308.

Hauk, P J., Wenzel, S. E., Trumble, A. E., Szefler, S. J., and D. Y. Leung. 1999. Increased T cell receptor Vbeta8+ T cells in bronchoalveolar lavage fluid of subjects with poorly controlled asthma: a potential role for microbial superantigens. J. Allergy Clin. Immunol. 104:37-45.

Heath, R. R., Stagg, S., Xu, F., and M. A. McCrae. 1997. Mapping of the target antigens of the rotavirus-specific cytotoxic T cell response. J. Gen. Virol. 78:1065-1075.

Helgeland, L., Vaage, J. T., Rolstad, B., Midtveldt, T., and P Brandtzaeg. 1996. Microbial colonization influences composition and $\mathrm{T}$-cell receptor $\mathrm{V}$ beta repertoire of intraepithelial lymphocytes in rat intestine. Immunology. 89:494-501. 
Herremans, T. M., Reimerink, J. H., Buisman, A. M., Kimman, T. G., and M. P. Koopmans. 1999. Induction of mucosal immunity by inactivated poliovirus vaccine is dependent on previous mucosal contact with live virus. J. Immunol. 162:5011-5018.

Hershberg, R. M., and L. F. Mayer. 2000. Antigen processing and presentation by cellsintestinal epithelial polarity and complexity. Immunol. Today. 21: 123-128.

Heyman, M., Corthier, G., Petit, A., Meslin, J. C., Moreau, C., and J.F. Desjeux. 1987. Intestinal absorption of macromolecules during viral enteritis: and experimental study on rotavirus-infected and germ-free mice. Pediatr. Res. 22:72-78.

Hoffman, L. M., Hogan, K. T., and L. W. Cashdollar. 1996. The reovirus nonstructural protein sigma1NS is recognized by murine cytotoxic T lymphocytes. J. Virol. 70:8160-8164.

Hooper, D. C., Rubin, D. H., and J. J. Cebra. 1994. Spontaneous proliferation of Peyer's patch cells in vitro. Int. Immunol. 6:873-880.

Hou, S., Hyland, L., Ryan, K. W., Portner, A., and P. C. Doherty. 1994. Virus-specific CD8+ Tcell memory determined by clonal burst size. Nature. 369:652-654.

Horie, Y., Chiba, M., Suzuki, T., Kudo, Kamata, A., Iizuka, M., and O. Masamune. 1998. Induction of the major histocompatibility class II antigens on human colonic epithelium by interferon-gamma, tumor necrosis factor-alpha, and Interleukin-2. J. Gastroenterol. 33:39-47. 
Horwitz, M. S., and N. Starvenick. 1999. Viruses, host responses, and autoimmunity. Immunol. Rev. 169:241-253.

Huang, F. D., Platt, N., Wykes, M., Major, J. R., Powell, T. J., Jenkins, C. D., and G. G. MacPherson. 2000. A discrete subpopulation of dendritic cells transports apoptotic intestinal epithelial cells to the T cell areas of the mesenteric lymph nodes. J. Exp. Med. 191:435-444.

Huard, B., and K. Fruh. A role for MHC class I down-regulation in the NK cell lysis of herpes virus-infected cells. Eur. J. Immunol. 30:509-515.

Imaoka, A., Matsumoto, S., Setoyama, H., Okada, Y., and Y. Umesaki. 1996. Proliferative recruitment of intestinal intraepithelial lymphocytes after microbial colonization of germ-free mice. Eur. J. Immunol. 26:945-948.

Inagaki-Ohara, K., Nishimura, H., Mitani, A., and Y. Yoshikai. 1997. Interleukin-15

preferentially promotes the growth of intestinal intraepithelial lymphocytes bearing gamma delta T cell receptor in mice. Eur. J. Immunol. 27:2885-2891.

Ishigama, T., White, C. A., and M. P. Pender. 1998. Soluble antigen therapy induces apoptosis of autoreactive $\mathrm{T}$ cells preferentially in the target organ rather than the peripheral lymphoid organs. Eur. J. Immunol. 28:1626-1635. 
Ishikawa, H., Saito, H., Suzuki, K., Oida, T., and Y. Kanamori. 1999. New gut-associated lymphoid tissue cryptopatches breed murine intestinal intraepithelial T cell precursors. Immunol. Res. 20:243-250.

Itoh, K., and S. Hirohata. 1995. The role of IL-10 in human B cell activation, proliferation, and differentiation. J. Immunol. 154:4341-4350.

Iwasaki, A., and B. L. Kelsall. 1999. Freshly isolated Peyer's patch, but not spleen, dendritic cells produce Interleukin-10 and induce the differentiation of T helper Type 2 cells. J. Exp. Med. 190:229-239.

Iwasaki, A., and B. L. Kelsall. 2000. Localization of distinct Peyer's patch dendritic cell subsets and their recruitment by chemokines macrophage inflammatory protein (MIP)-3a, MIP-3b, and secondary lymphoid organ chemokine. J. Exp. Med. 191:1381-1393.

Jameson, J., Cruz, J., and F. A. Ennis. 1998. Human cytotoxic T-lymphocyte repertoire to influenza A viruses. J. Virol. 72:8682-8689.

Jarry, A., Cerf-Bensussan, N., Brousse, N., Selz, F., and D. Guy-Grand. 1990. Subsets of CD3+ ( $\mathrm{T}$ cell receptor alpha/beta or gamma/delta) and CD3- lymphocytes isolated from normal human gut epithelium display phenotypical features different from their counterparts in peripheral blood. Eur. J. Immunol. 20:1097-1103. 
Kees, U., and P. H. Kramer. 1984. Most influenza A virus-specific memory cytotoxic T lymphocytes react with antigenic epitopes associated with internal virus determinants. $J$. Exp. Med. 159:365-377.

Kelsall, B. L., and W. Strober. 1996. Distinct populations of dendritic cells are present in the subepithelial dome and T cell regions of the murine Peyer's patch. J. Exp. Med. 183:237-247.

Kim, S. K., Reed, D. S., Olson, S., Schnell, M. J., Rose, J. K., Morton, P. A., and L. Lefrancois. 1998. Generation of mucosal cytotoxic T cells against soluble protein by tissue-specific environmental and costimulatory signals. Proc. Natl. Acad. Sci. U.S.A. 95:10814-10819.

Kim, S. K., Schluns, K. S., and L. Lefrancois. 1999. Induction and visualization of CD8 T cells following systemic virus infection. J. Immunol. 163:4125-4132.

Klein, J. R., and M. F. Kagnoff. 1984. Nonspecific recruitment of cytotoxic effector cells in the intestinal mucosal of antigen-primed mice. J. Exp. Med. 160:1931-1936.

Klein, J.R. 1986. Ontogeny of the Thy-1-, Lyt-2+ murine intestinal intraepithelial lymphocyte. J. Exp. Med. 164:309-314.

Koga, T., McGhee, J. R. Kato, H. Kato, R. Kiyono, H. and K. Fujihashi. 2000. Evidence for early ageing in the mucosal immune system. J. Immunol. 165:5352. 
Kronenberg, M., Brossay, L., Kurepa, Z., and J. Forman. 1999. Conserved lipid and peptide presentation functions of nonclassical class I molecules. Immunol. Today. 20:515-520.

Kuklin, N. A., Daheshia, M., Marconi, P. C., Krisky, D. M., Rouse, R. J. D., Glorioso, J. C., Manican, E., and B. T. Rouse. 1998. Modulation of mucosal and systemic immunity by enteric administration of nonreplicating herpes simplex virus expressing cytokines. Virology. 240:245253.

Lalvani, A., Brookes, R., Hambleton, S., Britton, W. J., Hill, A. V., and A. J. McMichael. 1997. Rapid effector function of CD8+ memory T cells. J. Exp. Med. 186:859-865.

Lambkin, R., McLain, L., Jones, S.E., Aldridge, S. L., and N. J. Dimmock. 1994. Neutralization escape mutants of type A influenza are readily selected by antisera from mice immunized with whole virus: a possible mechanism for antigenic drift. J. Gen. Virol. 75:3493-3502.

Lambrecht, B., Npauwels, R. A., and B. Fazekas De St. Groth. 2000. Induction of rapid T cell activation, division, and recirculation by intratracheal injection of dendritic cells in a TCR transgenic model. J. Immunol. 164:2937-2946.

Laufer, T. M., Glimcher, L. H., and D. Lo. 1999. Using thymic anatomy to dissect T cell repertoire selection. Semin. Immunol. 11:65-70. 
Lee, H. M., and S. Rich. 1993. Differential activation of CD8+ T cells by transforming growth factor-beta 1. J. Immunol. 151:668-677.

Lefrancois, L., Olson, S., and D. Manopust. 1999. A critical role for CD40-CD40 ligand interactions in the amplification of the mucosal CD8 T cell response. J. Exp. Med. 190: 12751284.

Lefrancois, L., Parker, C.M., Olson, S., Muller, W., Wagner, N., Schon, M.P., and L. Puddington. 1999. The role of beta 7 integrins in CD8 T cell trafficking during an antiviral immune response. J. Exp. Med. 189:1631-1638.

Lefrancois, L., Altman, J.D., Williams, K., and S. Olson. 2000. Soluble antigen and CD40 triggering are sufficient to induce primary and memory cytotoxic T cells. J. Immunol. 164: 725732.

Leitie-de-Moraes, M. C., Hameg, A., Arnould, A., Machavoine, F., Koezuka, Y., Schneider, E., Herbelin, A., and M. Dy. 1999. A distinct IL-18-induced pathway to fully activate NK T lymphocytes independently from TCR engagement. J. Immunol. 163:5871-5876.

Lillard, J. W., Boyaka, P. N., Hedrick, J.A., Zlotnik, A., and J. R. McGhee. 1999. Lymphotactin acts as an innate mucosal adjuvant. J. Immunol. 162:1959-1965.

Lin, M. Y., and R. M. Welsh. 1998. Stability and diversity of T cell receptor repertoire usage during lymphocytic choriomeningitis virus infection of mice. J. Exp. Med. 188:1993-2005. 
Lin, T., Matsuzaki, G., Yoshida, H., Kenai, H., Omoto, K., Umesue, M., Singaram, C., and K. Nomoto. 1996. Thymus ontogeny and the development of TCR $\alpha \beta$ intestinal intraepithelial lymphocytes. Cell. Immunol. 171:132-139.

Liu, L. M., and G. G. MacPherson. 1991. Lymph-borne (veiled) dendritic cells can acquire and present intestinally administered antigens. Immunology. 73:281-286.

Liu, L. M., and G. G. MacPherson. 1993. Antigen acquisition by dendritic cells: intestinal dendritic cells acquire antigen administered orally and can prime naïve T cells in vivo. J. Exp. Med. 177:1299-1307.

Liu, X. S., Abdul-Jabbar, I., Qi, Y. M., Frazer, I.H., J. Zhou. 1998. Mucosal immunization with papillomavirus virus-like particles elicits systemic and mucosal immunity in mice. Virology. 252:39-45.

Liu, Z., Geboes, K., Colpaert, S., D’Haens, G. R., Rutgeerts, P., and J. L. Ceuppens. 2000. IL-15 is highly expressed in inflammatory bowel disease and regulates local T cell-dependent cytokine production. J. Immunol. 164:3608-3615.

Livingstone, A. M., and M. Kuhn. 1999. Dendritic cells need T cell help to prime cytotoxic T cell responses to strong antigens. Eur. J. Immunol. 29:2826-2834. 
Logan, A.C., Chow, K. N., George, A., Weinstein, P. D., J. J. Cebra. 1991. Use of Peyer's patch and lymph node fragment cultures to compare local immune response to Morganella morganii. Infect. Immun. 59:1024-1031.

London, S. D., Rubin, D. H., and J. J. Cebra. 1987. Gut mucosal immunization with reovirus serotype 1/L stimulates virus-specific cytotoxic T cell precursors as well as IgA memory cells in Peyer's patches. J. Exp. Med. 165:830-847.

London, S. D., Cebra, J. J., and D. H. Rubin. 1989. Intraepithelial lymphocytes contain virusspecific, MHC-restricted cytotoxic cell precursors after gut mucosal immunization with reovirus serotype 1/Lang. Reg. Immunol. 2:98-102.

Lopes, L. M., Hughson, E., Anstee, Q., O’neil, D., Katz, D. R., and B. M. Chain. 1999. Vectorial function of human major histocompatibility complex class II in a human intestinal cell line. Immunology. 98:16-26.

Lundqvist, C., Melgar, S., Yeung, M. M., Hammarstrom, S., and M. L. Hammarstrom. 1996. Intraepithelial lymphocytes in human gut have lytic potential and a cytokine profile that suggests T helper 1 and cytotoxic functions. J. Immunol. 157:1926-1934.

Lynne, J. E., Schmid, I., Matud, J. L., Hirji, K., Buessow, S., Shlian, D. M., and J. V. Giorgi. 1998. Major expansions of select CD8+ subsets in acute Epstein-Barr virus infection: Comparison with chronic human immunodeficiency virus disease. J. Infect. Dis. 177:1083-1087. 
MacDonald, T. T., Bajaj-Elliot, M., and S. L. F. Pender. 1999. T cells orchestrate intestinal mucosal shape and integrity. Immunol. Today. 20:505-510.

Mackay, C. R., Marston, W. L., and L. Dudler. 1990. Naïve and memory T cells show distinct pathways of lymphocyte recirculation. J. Exp. Med. 171:801-817.

Mackay, C. R., Andrew, D. P., Brisken, M., Ringeler, D. J., E. C. Butcher. 1996. Phenotype, and migration properties of three major subsets of tissue homing T cells in sheep. Eur. J. Immunol. 26:2433-2439.

Major, A. S., and C. F. Cuff. 1996. Effects of the route of infection on immunoglobulin G subclasses and specificity of the reovirus-specific humoral immune response. J. Virol. 70:59685974.

Mathy, N. L., Scheuer, W., Lanzendorfer, M., Honold, K., Ambrosius, D., Norley, S., and R. Kurth. 2000. Interleukin-16 stimulates the expression and production of pro-inflammatory cytokines by human monocytes. Immunology. 100:63-69.

Matloubian, M., Concepcion, R. J., and R. Ahmed. CD4+ T cells are required to sustain CD8+ cytotoxic T-cell responses during chronic viral infection. J. Virol. 68:8056-8063. 
Mayrhofer, G. 1980. Thymus-dependent and thymus-independent subpopulations of intestinal intraepithelial lymphocytes: a granular subpopulation of probable bone-marrow origin and relationship to mucosal mast cells. Blood. 55:532-535.

McAdam, A. J., Schweitzer, A. N., and A. H. Sharpe. 1998. The role of B7 co-stimulation in activation and differentiation of CD4+ and CD8+ T cells. Immunol. Rev. 165:231-247. McGhee, J.R., Fujihashi, K., Beagley, K.W., and H. Kiyono. 1991. Role of Interleukin-6 in human and mouse mucosal IgA plasma cell responses. Immunol. Res. 10:418-422.

Mehrota, P. T., Grant, A. J., and J. P. Siegel. 1995. Synergistic effects of IL-7 and IL-12 on human T cell activation. J. Immunol. 154:5093-5102.

Mengel, J., Cardillo, F., Aroeira, L. S., Williams, O., Russo, M., and N. M. Vaz. 1995. Anti- $\gamma \delta$ T cell antibody blocks the induction and maintenance of oral tolerance to ovalbumin in mice. Immunol. Lett. 48:97-102.

Merritt, A. J., Potten, C. S., Watson, A. J.M., Loh, D. Y., Nakayama, K., Kakayama, K., and J. A. Hickman. 1995. Differential expression of Bcl-2 in intestinal epithelia. Correlation with attenuation of apoptosis in colonic crypts and the incidence of colonic neoplasia. J. Cell Sci. 108:2261-2271.

Milligan, G. N., and D. I. Bernstein. 1997. Interferon- $\gamma$ enhances resolution of herpes simplex virus type 2 infection of the murine genital tract. Virology. 229:259-268. 
Mitchell, T., Kappler, J., and P. Marrack. 1999. Bystander virus activation prolongs activated T cell survival. J. Immunol. 162:4527-4535.

Moghaddami, M., Cummins, A., and G. Mayerhofr. 1998. Lymphocyte-filled villi: comparisons with other lymphoid aggregations in the mucosal of the human small intestine. Gastroenterology. 115:1414-1425.

Molberg, O., Nilsen, E. M., Sollid, L. M., Scott, H., Brandtzaeg, P., Thorsby, E., and K. E. A. Lundin. 1998. CD4+ T cells with specific reactivity against astrovirus isolated from normal human small intestine. Gastroenterology. 114:115-122.

Mosley, R.L., Whetsell, M., Stickney, D., Whetsell, L., Schaefer, F.V., Miller, K.S., and J.R. Klein. 1994. Phenotype and TCR gamma delta repertoire of intestinal intraepithelial lymphocytes of wild mice (Mus musculus domesticus): abundance of V gamma 1 transcripts and extensive delta gene diversity. Int. Immunol. 6:231-238.

Mongkolsapaya, J., Jaye, A., Callan, M. F., Magnusen, A. F., McMichael, A. J., and H. C. Whittle. 1999. Antigen-specific expansion of cytotoxic T lymphocytes in acute measles virus infection. J. Virol. 73:67-71.

Montgomery, P. C., Lemaitre-Coelho, I. M., and J. P. Vaerman. 1980. A common mucosal immune system. Antibody expression in secretions following gastrointestinal stimulation. Immunol. Commun. 9:705-713. 
Moodycliffe, A. M., Shreedhar, V., Ullrich, S. E., Walterscheid, J., Bucana, C., Kripke, M. L., and L. Flores-Romo. 2000. CD40-CD40 ligand interactions in vivo regulate migration of antigen-bearing dendritic cells from the skin to draining lymph nodes. J. Exp. Med. 191:20112020.

Muller, S., Buhler-Jungo, M., and C. Mueller. 2000. Intestinal intraepithelial lymphocytes exert potent protective cytotoxic activity during an acute viral infection. J. Immunol. 164:1986-1994.

Muir, W. I., Bryden, W. L., and A. J. Husband. 1998. Evaluation of the efficacy of intraperitoneal immunization in reducing salmonella typhimurium infection in chickens. Poult. Sci. $77: 1874-1883$.

Murali-Krishna, K., Altman, J. D., Suresh, M., Sourdive, D. J., Zajac, A. J., Miller, J. D., Slansky, J., and R. Ahmed. 1998. Counting antigen-specific CD8+ T cells: a reevaluation of bystander activation during viral infection. Immunity. 8:177-187.

Murali-Krishna, K., Altman, J. D., Suresh, M., Sourdive, D. J., Zajac, A. J., and R. Ahmed. 1998. In vivo dynamics of anti-viral CD8 T cell responses to different epitopes. An evaluation of bystander activation in primary and secondary responses to viral infection. Adv. Exp. Med. Biol. 452:123-142. 
Nanno, M., Matsumoto, S., Koike, R., Miyasaka, M., Kawaguchi, M., Masuda, T., Miyawaki, S., Cai, Z., Shimamura, T., and Y. Fujiura. 1994. Development of intestinal intraepithelial T lymphocytes is independent of Peyer's patches and lymph nodes in aly mutant mice. J. Immunol. 153:2014-2020.

Naumov, Y. N., Hogan, K. T., Naumova, E. N., Pagel, J. T., and J. Gorski. 1998. A class I MHCrestricted recall response to a viral peptide is highly polyclonal despite stringent CDR3 selection: Implications for establishing memory T cell repertoires in "real-world" conditions. J. Immunol. 160:2842-2852.

Newberry, R. D., Stenson, W. F., and R. G. Lorenz. 1999. Cyclooxygenase-2-dependent arachidonic acid metabolites are essential modulators of the intestinal immune response to dietary antigen. Nat. Med. 5:900-906.

Nguyen, H. H., van Ginkel, F. W., Vu, H. L., Novak, M. J., McGhee, J. R., and J. Mestecky. 2000. Gamma interferon is not required for mucosal cytotoxic T lymphocyte responses or heterosubtypic immunity to influenza A infection in mice. J. Virol. 74:5495-5501.

Oehen, S., and K. Brduscha-Riem. 1998. Differentiation of naïve CTL to effector and memory CTL: correlation of effector function with phenotype and cell division. J. Immunol. 161:53385346. 
Offit, P. A., and K. I. Dudzik. 1989. Rotavirus-specific cytotoxic T lymphocytes appear in the intestinal mucosal surface after rotavirus infection. J. Virol. 63:3507-3512.

Offit, P.A., Cunningham, S. L., and K. I. Dudzik. 1991. Memory and distribution of virusspecific cytotoxic T lymphocytes (CTL) and CTL precursors of rotavirus infection. J. Virol. 65:1318-1324.

Oida, T., Suzuki, K., Nanno, M., Kanamori, Y., Saito, H., Kubota, E., Kato, S., Itoh, M., Kaminogawa, S., and H. Ishikawa. 2000. Role of gut cryptopatches in early extrathymic maturation of intestinal intraepithelial T cells. J. Immunol. 164:3616-3626.

Okamoto, I., Kohno, K., Tanimoto, T., Ikegami, H., and M. Kurimoto. 1999. Development of CD8+ effector T cells is differentially regulated by IL-18 and IL-12. J. Immunol. 162:3202-3211.

Opferman, J. T., Ober, B. T., and P. G. Ashton-Rickardt. 1999. Linear differentiation of cytotoxic effectors into memory T lymphocytes. Science. 283:1745-1748.

Paganin, C., Frank, I., and G. Trinchieri. 1995. Priming for high interferon-gamma production induced by Interleukin-12 in both CD4+ and CD8+ T cell clones from HIV-infected patients. $J$. Clin. Invest. 96:1677-1682. 
Pal, S., Peterson, E. M., and L. M., de la Maza. 1996. Intranasal immunization induces long-term protection in mice against a Chlamydia trachomatis genital challenge. Infect. Immun. 64:53415348.

Park, S., Roark, J. H., and A. Bendelac. 1998. Tissue-specific recognition of mouse CD1 molecules. J. Immunol. 160:3128-3134.

Parr, M. B., and E. L. Parr. 2000. Interferon-g up-regulates intercellular adhesion molecule-1 and vascular cell adhesion molecule-1 and recruits lymphocytes into the vagina of immune mice challenged with herpes simplex virus-2. Immunology. 99:540-545.

Parrott, D. M., Tait, C., MacKenzie, S., Mowat, A. M., Davies, M. D., and H. S. Micklem. 1983. Analysis of the effector functions of different populations of mucosal lymphocytes. Ann. N.Y. Acad. Sci. 409:307-320.

Perez, L., and L. Carrasco. 1994. Involvement of the vacuolar H(+) ATPase in animal virus entry. J. Gen. Virol. 75:2595-2606.

Pewe, L., Xue, S., and S. Perlman. 1997. Cytotoxic T-cell-resistant variants arise at early times after infection in C57BL/6 but not in SCID mice infected with a neurotropic coronavirus. $J$. Virol. 71:7640-7647. 
Podolsky, D. K. 2000. Mechanisms of regulatory peptide action in the gastrointestinal tract: trefoil peptides. Gastroenterology. 35:69-74.

Poussier, P., and M. Julius. 1999. Speculations on the lineage relationships among CD4(-)8(+) gut-derived T cells and their role(s). Semin. Immunol. 11:293-303.

Premier R. R., Jacobs, H. J., Brandon, M. R., and E. N. Meeusen. 1996. Distribution of antigenspecific memory $\mathrm{T}$ cells in lymph nodes after immunization at peripheral or mucosal sites. Immunol. Cell Biol. 74:265-273.

Ratner, A and W. R. Clark. 1993. Role of TNF-alpha in CD8+ cytotoxic T lymphocytesmediated lysis. J. Immunol. 150:4303-4314.

Redchenko, I. V., and A.B. Rickenson. 1999. accessing Epstein-Barr virus specific T-cell memory with peptide-loaded dendritic cells. J. Virol. 73:334-342.

Regnault, A., Cumano, A., Vassalli, P., Guy-grand, D., and P. Kourilsky. 1994. Oligoclonal repertoire of the CD8 alpha alpha and the CD8 alpha beta TCR alpha/beta murine intestinal intraepithelial T lymphocytes: evidence for the random emergence of T cells. J. Exp. Med. $180: 1345-1358$.

Regnault, A., Kourilsky, P., and A. Cumano. 1995. The TCR-beta chain repertoire of gut-derived T lymphocytes. Semin. Immunol. 7:307-319. 
Regnault, A., Levraud, J.P., Lim, A., Six, A., Moreau, C., Cumano, A., and P. Kourilsky. 1996. The expansion and selection of $\mathrm{T}$ cell receptor alpha beta intestinal intraepithelial $\mathrm{T}$ cell clones. Eur. J. Immunol. 26:914-921.

Regnault, A., Lankar, D., Lacabanne, V., Rodriguez, A., Thery, C., Rescigno, M., Saito, T., Verbeek, S., Bonnerot, C., Ricciardi-Castagnoli, P., and S. Amigorena. 1999. Fcgamma receptormediated induction of dendritic cell maturation and major histocompatibility complex class Irestricted antigen presentation after immune complex internalization. J. Exp. Med. 189:371-380.

Roberts, S. J., Smith, A. L., West, A. B., Wen, L., Findly, R. C., Owen, M. J., and A. C. Hayday. 1996. T-cell alpha-beta + and gamma-delta + deficient mice display abnormal but distinct phenotypes toward a natural, widespread infection of the intestinal epithelium. Proc. Natl. Acad. Sci. U.S.A. 93:11774-11779.

Rocha, B., Vassalli, P., and D. Guy-Grand. 1994. Thymic and extrathymic origins of gut intraepithelial lymphocyte populations in mice. J. Exp. Med. 180:681-686.

Rocha, B., Vassalli, P., and D. Guy-Grand. 1991. The V beta repertoire of mouse gut homodimeric alpha CD8+ intraepithelial $\mathrm{T}$ cell receptor alpha/beta + lymphocytes reveals a major extrathymic pathway of T cell differentiation. J. Exp. Med. 173:483-486. 
Roth, M. G., Doyle, C., Sambrook, J., and M. J. Gething. 1986. Heterologous transmembrane and cytoplasmic domains direct functional chimeric influenza virus hemagglutinins into the endocytic pathway. J. Cell. Biol. 102:1271-1283.

Rowbottom, A. W., Lepper, M. W., Garland, R. J., Cox, C. V., Corley, E. G., Oakhill, A., and C.G. Steward. 1999. Interleukin-10-induced CD8 cell proliferation. Immunology. 98:80-89. Rubin, D.H., Kornstein, M.J., and A.O. Anderson. 1985. Reovirus serotype 1 intestinal infection: a novel replicative cycle with ileal disease. J. Virol. 53:391-398.

Russo, V., Zhou, D., Sartirana, C., Rovere, P., Villa, A., Rossini, S., Traversari, C., and C. Bourdignon. 2000. Acquisition oh intact allogenic human leukocyte antigen molecules by human dendritic cells. Blood. 95:3473-3477.

Santamaria., B. L. F., Moser, R., Perez, S. M. T., Picker, L .J., Blaser, K., and C. Hauser. 1995. Migration of skin-homing $\mathrm{T}$ cells across cytokine-activated human endothelial cell layers involves interaction of the cutaneous lymphocyte-associated antigen (CLA), the very late antigen-4 (VLA-4), and the lymphocyte function-associated antigen-1 (LFA-1). J. Immunol. 154:1543-1550.

Saito, H., Kanamori, Y., Takemori, T., Nariuchi, H., Kubota, E., Takahashi-Iwanaga, H., Iwanaga, T., and H. Ishikawa. 1998. Generation of intestinal T cells from progenitors residing in gut cryptopatches. Science. 280:275-278. 
Samuel, C. E. 1998. Reoviruses and the interferon system. Curr. Top. Microbiol. Immunol. 233:125-145.

Santin, A. D., Hermonat, P. L., Ravaggi, A., Bellone, S., Pecorelli, S., Roman, J. J., Parham, G. P., and M. J. Cannon. 2000. Interleukin-10 increases Th1 cytokine production and cytotoxic potential in human papillomavirus-specific CD8+ cytotoxic T lymphocytes. J. Virol. 74:47294737.

Sarin, A., Nakajima, H., and P.A. Henkart. 1995. A protease-dependent TCR-induced pathway in mature lymphocytes. J. Immunol. 154:5806-5812.

Schuhbauer, D. M., Mitchison, N. A., and B. Mueller. 2000. Interactions within clusters of dendritic cells and helper T cells during initial Th1/Th2 commitment. Eur. J. Immunol. 30:12551262.

Schmucker, D. L., Heyworth, M. F., Owen, R. L., and Daniels, C.K. 1996. Impact of aging on gastrointestinal mucosal immunity. Dig. Dis. Sci. 41:1183-1193.

Schwartz, R.H. 1997. T cell clonal anergy. Curr. Opin. Immunol. 9:351-357.

Sechoy, O., Vidal, M., Philippot, J.R., and A. Bienvenue. 1989. Interactions of human lymphoblasts with targeted vesicles containing Sendai virus envelope proteins. Exp. Cell. Res. 185:122-131. 
Selin, L. K., Nahill, S. R., and R. M. Welsh. 1994. Cross-reactivities in memory cytotoxic T lymphocyte recognition of heterologous viruses. J. Exp. Med. 179:1933-1943.

Selin, L. K., Vergilis, K., Welsh, R. M., and S. R. Nahill. 1996. Reduction of otherwise remarkably stable virus-specific cytotoxic T lymphocyte memory by heterologous viral infections. J. Exp. Med. 183:2489-2499.

Selin, L. K., Lin, M. Y., Kraemer, K. A., Pardoll, D. M., Schneck, J. P., Varga, S. M., Santolucito, P. A., Pinto, A. K., and R. M. Welsh. 1999. Attrition of T cell memory: Selective loss of LCMV epitope-specific memory CD8 T cells following infections with heterologous viruses. Immunity.

11:733-742.

Sewell, A. K., Price, D. A., Teisserenc, H., Booth, B. L., Gileadi, U., Flavin, F.M., Trowsdale, J., Phillips, R.E., and V. Cerundolo. 1999. IFN-gamma exposes a cryptic cytotoxic T lymphocyte epitope in HIV-1 reverse transcriptase. J. Immunol. 162:7075-7079.

Sharp, M., Terada, K., Wilson, A., Nader, S., Kinchington, P.E., Ruyechan, W.T., Hay, J., and A.M. Arvin. 1992. Kinetics and viral protein specificity of the cytotoxic T lymphocyte response in healthy adults immunized with live attenuated varicella vaccine. J. Infect. Dis. 165-852-858.

Shawar, S. M., Vyas, J. M., Rodgers, J. R., and R. R. Rich. 1994. Antigen presentation by major histocompatibility complex class I-B molecules. Annu. Rev. Immunol. 12:839-880. 
She, J., Matsui, K., Terhorst, C., and S.T. Ju. 1998. Activation-induced apoptosis of mature T cells is dependent upon the level of surface TCR but not on the presence of the CD3 zeta ITAM. Int. Immunol. 10:1733-1740.

Sher, A., Fiorentino, D., Caspar, P., Pearnce, E., and T. Mossman. 1991. Production of IL-10 by CD4+ T lymphocytes correlates with down-regulation of Th1 cytokine synthesis in helminth infection. J. Immunol. 147:2713-2716.

Shi, L., Mai, S., Israels, S., Browne, K., Trapani, J.A., and A.H. Greenberg. 1997. Granzyme B (GraB) autonomously crosses the cell membrane and perforin initiates apoptosis and GraB nuclear localization. J. Exp. Med. 185:855-866.

Silins, S. L., Cross, S. M., Elliot, S. L., Pye, S. J., Burrows, S. R., Burrows, J. M., Moss, D. J., Argaet, V. P., and I. S. Misko. 1996. Development of Epstein-Barr virus-specific memory T cell receptor clonotypes in acute infectious mononucleosis. J. Exp. Med. 184:1815-1824.

Silins, S. L., Cross, S. M., Krauer, K. G., Moss, D. J., Schmidt, K. W., and I. S. Misko. 1998. A functional link for major TCR expansions in healthy adults caused by persistent Epstein-Barr virus infection. J. Clin. Invest. 102:1551-1558.

Smith, T. J., Terada, N., Robinson, C. C., and E. W. Gelfand. 1993. Acute infectious mononucleosis stimulates the selective expression/expansion of Vbeta 6.1-3 and Vbeta7 T cells. Blood. 81:1521-1526. 
Snijders, A., Kalinski, P., Hilkens, C. M., and M. L. Kapsenberg. 1998. High-level IL-12 production by human dendritic cells requires two signals. Int. Immunol. 10:1593-1598.

Soesatyo, M., Thepen, T., Ghufron, M., Biewenga, J., and T. Sminia. 1993. Peritoneal cell labeling: a study on the migration of macrophages and dendritic cells towards the gut. Adv. Exp. Med. Biol. 329:321-326.

Sonoda, E., Hitoshi, Y., Yamaguchi, N., Ishii, T., Tominaga, A., Araki, S., and K. Takatsu. 1992. Differential regulation of IgA production by TGF-beta and IL-5: TGF-beta induces surface IgApositive cells bearing IL-5 receptor, whereas IL- 5 promotes their survival and maturation into IgA-secreting cells. Cell. Immunol. 140:158-172.

Sourdive, D.J., Murali-Krishna, K., Altman, J. D., Zajac, A. J., Whitmire, J. K., Pannetier, C., Kourilsky, P., Evavold, B., Sette, A., and R. Ahmed. 1998. Conserved T cell receptor repertoire in primary and memory CD8 T cell responses to an acute viral infection. J. Exp. Med. 188:71-82.

Specht, C., Pauels, H. G., Becker, C., E. Kolsch. 1998. Differential activation of CD8+ tumorspecific Tc1 and Tc2 cells by an IL-10-producing murine plasmacytoma. Dev. Immunol. 6:331342.

Stenger, S., Hanson, D.A., Teitelbaum, R., Dewan, P., Niazi, K. R., Froelich, C. J., Ganz, T., Thoma-Uszynski, S., Melian, A., Bogdan, C., Porcelli, S.A., Bloom, B.R., Krensky, A.M., and 
R.L. Modlin. 1998. An antimicrobial activity of cytolytic T cells mediated by granulysin. Science. 282:121-125.

Strayer, D. S., and J.L. Liebowitz. 1987. Virus-lymphocyte interactions during the course of immunosuppressive virus infection. J. Gen. Virol. 68:463-472.

Strober, W., and B. Kelsall. 1998. To be response or not to be responsive, that is the mucosal question. Gastroenterology. 114:214-217.

Stumbels, P. A., Thomas, J. A., Pimm, C. L., Lee, P. T., Venaille, T. J., Proksch, S., and P. G. Holt. 1998. Resting respiratory tract dendritic cells preferentially stimulate T helper cell type 2 (Th2) responses and require obligatory cytokine signals for induction of Th1 immunity. J. Exp. Med. 188:2019-2031.

Sundstedt, A., Hoiden, I., Hansson, J., Hedlund, G., Kalland, T., and M. Dohlsten. 1995. Superantigen-induced anergy in cytotoxic CD8+ T cells. J. Immunol. 154:6306-6313.

Sundstedt, A., Grundstrom, S., and M. Dohlsten. 1998. T cell- and perforin-dependent depletion of B cells in vitro by staphylococcal enterotoxins A. Immunology. 95:76-82.

Swallow, M. M., Wallin, J.J., and W.C. Sha. 1999. B7h, a novel co-stimulatory homolog of B7.1 and B7.2, is induced by TNF-alpha. Immunity. 11:423-432. 
Sydora, B. C., Mixter, P. F., Holcombe, H. R., Eghtesady, P., Williams, K., Amaral, M. C., Nel, A., and M. Kronenberg. 1993. Intestinal intraepithelial lymphocytes are activated and cytolytic but do not proliferate as well as other T cells in response to mitogenic signals. J. Immunol. 150:2179-2191.

Sydora, B. C., Jamieson, B. D., Ahmed, R., and M. Kronenberg. 1996. Intestinal intraepithelial lymphocytes respond to systemic lymphocytic choriomeningitis virus infection. Cell. Immunol. 167:161-169.

Tarazona, R., Sponaas, A. M., Mavria, G., Zhou, M., Schulz, R., Tomlinson, P., Antoniou, J., and A.L. Mellor. 1996. Effects of different antigenic microenvironments on the course of CD8+ T cell responses in vivo. Int. Immunol. 8:351-358.

Thompson, A. H., London, L., and S.D. London. 1999. Heterogeneous cytokine production by acutely stimulated bronchoalveolar T lymphocytes in reovirus 1/Lang-infected mice. Cell. Immunol. 198:120-130.

Tietz, W., and A. Hamann. 1997. The migratory behavior of CD4+ cells of memory phenotype. Eur. J. Immunol. 27:2225-2232.

Trapani, J. A., Sutten, V. R., and M. J. Smyth. 1999. CTL granules: evolution of vesicles essential for combating virus infections. Immunol. Today. 20:351-356. 
Trimble, L. A., Xu, Z., and J. Lieberman. 1999. Clonal expansion of antigen-specific CD8+ cytotoxic T lymphocytes is regulated by late exposure to serum to prevent apoptosis. J. Immunol. Methods. 225:39-52.

Tripp, R. A., Hou, S., Mcmickle, A., Houston, J., and P. C. Doherty. 1995. Recruitment and proliferation of CD8+ T cells in respiratory virus infections. J. Immunol. 154:6013-6021.

Tripp, R. A., Hou, S., and P. C. Doherty. 1995. Temporal loss of the activated L-selectin-low phenotype for virus-specific CD8+ memory T cells. J. Immunol. 154:5870-5875. Tucek, C.L., Desaymard, C., and M. Papiernik. 1993. A kinetic study on the deletion of thymic, peripheral, and gut-associated $\mathrm{V}$ beta6 $+\mathrm{T}$ cells in an Mls-1b BALB/c colony infected with an exogenous mouse mammary tumor virus. J. Immunol. 151:759-766.

Uhnoo, I., Riepenhoff-Talty, M., Dharakul, T., Chegas, P., Fisher, J.E., Greenberg, J.B., and P.L. Ogra. 1990. Extramucosal spread and development of hepatitis in immunodeficient and normal mice infected with rhesus rotavirus. J. Virol. 64:361-368.

Usherwood, E. J., Hogan, R. J., Crowther, G., Surman, S. L., Hogg, T. L., Altman, J. D., and D. L. Woodland. 1999. Functionally heterogeneous CD8(+) T-cell memory is induced by Sendai virus infection of mice. J. Virol. 73:7278-7286. 
Van der Most, R.G., Murali-Krishna, K., Whitton, J. L., Oseroff, C., Alexander, J., Southwood, S., Sidney, J., Chesnut, R. W., Sette, A., and R. Ahmed. 1998. Identification of the Db- and Kbrestricted subdominant cytotoxic T-cell responses in lymphocytic choriomeningitis virus-infected mice. Virology. 240-158-167.

Van Kerckhove, C., Russell, G. J., Deusch, K., Reich, K., Bhan, A. K., DerSimonian, H., and M. B. Brenner. 1992. Oligoclonality of human intestinal intraepithelial T cells. J. Exp. Med. 175:5763.

VanCott, J. L., Franco, M. A., Greenberg, H. B., Sabbaj, S., Tang, B., Murray, R., and J. R. McGhee. 2000. Protective immunity to rotavirus shedding in the absence of Interleukin-6: Th1 cells and immunoglobulin A develop normally. J. Virol. 74:5250-5256.

Van Houten, N., Mixter, P. F., Wolfe, J., and R. C. Budd. 1993. CD2 expression on murine intestinal intraepithelial lymphocytes is bimodal and defines proliferative capacity. Int. Immunol. 5:665-672.

Van Vlasselaer, P., Punnonen, J., and J.E. de Vries. 1992. transforming growth factor beta directs IgA switching in human B cells. J. Immunol. 148:2062-2067.

Vasseur, F., Le Campion, A., Pavlovitch, J. H., and C. Penit. 1999. Distribution of cycling T lymphocytes in blood and lymphoid organs during immune responses. J. Immunol. 162:51645172. 
Verdin, E. M., King, J. L., and E. Maratos-Flier. 1989. Characterization of a comm0on highaffinity receptor for reovirus serotypes 1 and 3 on endothelial cells. J. Virol. 63: 1318-1325.

Vieira, P. L., de Jong, E. C., Wierenga, E. A., Kapsenberg, M. L., and P. Kalinsky. 2000. Development of Th1-inducing capacity in myeloid dendritic cells requires environmental instruction. J. Immunol. 164:4507-4512.

Vijh, S., and E. G. Pamer. 1997. Immunodominant and subdominant CTL responses to Listeria monocytogenes infection. J. Immunol. 158:3366-3371.

Vijh, S., Pilip, I. M., and E. G. Pamer. 1999. Noncompetitive expansion of cytotoxic T lymphocytes specific for different antigens during bacterial infection. Infect. Immun. 67:13031309.

Viney, J. L., Kilshaw, P. J., and T. T. MacDonald. 1990. Cytotoxic alpha/beta+ and gamma/delta+ Tcells in the murine intestinal epithelium. Eur. J. Immunol. 20:1623-1626.

Von Herrath, M. G., Yokoyama, M., Dockter, J., Oldstone, M. B., and J. L. Whitton. 1996. CD4deficient mice have reduced levels of memory cytotoxic $\mathrm{T}$ lymphocytes after immunization and show diminished resistance to subsequent virus challenge. J. Virol. 70:1072-1079.

Wack, A., Corbella, P., Harker, N., Crispe, I. N., and D. Kioussis. 1997. Multiple sites of postactivation CD8+ T cell disposal. Eur. J. Immunol. 27:577-583. 
Wade, T., Bill, J., Marrack, P.C., Palmer, E., and J. W. Kappler. 1988. Molecular basis for the non-expression of V $\beta 17$ in some strains of mice. J. Immunol. 141:2165-2167.

Weinstein, P. D., and J. J. Cebra. 1991. The preference for switching to IgA expression by Peyer's patch germinal center B cells is likely due to the intrinsic influence of their microenvironment. J. Immunol. 147:4126-4135.

Welsh, R. M., and J. M. McNally. 1999. Immune deficiency, immune silencing, and clonal exhaustion of T cell responses during viral infections. Curr. Opin. Microbiol. 2:382-387. Westermann, J., and U. Bode. 1999. Distribution of activated T cells migrating through the body: a matter of life and death. Immunol. Today. 20:302-306.

Wherry, E. J., Puorro, K. A., Porgador, A., and L. C. Eisenlohr. 1999. The induction of virusspecific CTL as a function of increasing epitope expression: responses rise steadily until excessively high levels of epitope are attained. J. Immunol. 163:3735-3745.

Whitmire, J. K., and R. Ahmed. 2000. Costimulation in antiviral immunity: differential requirements for CD4(+) and CD8(+) T cell responses. Curr. Opin. Immunol. 12:448-455.

Williams, M. B., and E. C. Butcher. 1997. Homing or naïve and memory T lymphocyte subsets to Peyer's patches, lymph nodes and spleen. J. Immunol. 159:1746-1752. 
Williams, N. A., Wilson, A. D., Bailey, M., Bland, P. W., and C. R. Stokes. 1992. Primary antigen-specific $\mathrm{T}$-cell proliferative responses following presentation of soluble protein antigen by cells from the murine small intestine. Immunology. 75:608-613.

Wilson, J. D., Cranage, M., Cook, N., Leech, S., McMichael, A. J., and M. F. Callan. 1998. Evidence for the persistence of monoclonal expansions of $\mathrm{CD} 8+\mathrm{T}$ cells following primary simian immunodeficiency virus infection. Eur. J. Immunol. 28:1172-1180.

Witmer, M. D., and R. M. Steinman. 1984. The anatomy of peripheral lymphoid organs with emphasis on accessory cells" light-microscopic immunocytochemical studies of mouse spleen, lymph node, and Peyer's patch. Am. J. Anat. 170:465-481.

Wolf, J. L., Rubin, D. H., Finberg, R., Kauffman, R. S., Sharpe, A. H., Trier, J. S., and B.N. Fields. 1983. Intestinal M cells: a pathway for entry of reovirus into the host. Science. 212:471472.

Yamamoto, M., Fujihashi, K., Beagley, K. W., McGhee, J. R., and H. Kiyono. 1993. Cytokine synthesis by intestinal intraepithelial lymphocytes. Both gamma/delta $\mathrm{T}$ cell receptor-positive and alpha/beta $\mathrm{T}$ cell receptor-positive T cells in the G1 phase of the cell cycle produce IFNgamma and IL-5. J. Immunol. 150:106-114.

York, I. A., Goldberg, A. L., Mo, X. Y., and K. L. Rock. 1999. Proteolysis and class I major histocompatibility complex antigen presentation. Immunol. Rev. 172:49-66. 
Yoshikai, Y. 1999. The interaction of intestinal epithelial cells and intraepithelial lymphocytes in host defense. Immunol. Res. 20:219-235.

Zhang, X., Sun, S., Hwang, I., Tough, D. F., and J. Sprent. 1998. Potent and selective stimulation of memory-phenotype CD8+ T cells in vivo by IL-15. Immunity. 8:591-599. 


\title{
The CTL V $\beta$ Repertoire Induced Following Oral or Systemic Infection with Reovirus
}

\author{
Jonathan R. Fulton and Christopher F. Cuff \\ Department of Microbiology and Immunology, \\ West Virginia University School of Medicine
}




\begin{abstract}
Reovirus type 1 strain Lang (T1L) induces both humoral and cell-mediated immune responses in the draining lymph nodes of parenterally infected mice and the Peyer's patches of orally infected mice. Distinct mechanisms of viral protein processing and epitope generation, naïve $\mathrm{CD}^{+} \mathrm{T}$ cell recruitment, or other micro-environmental influences might influence the magnitude and $\mathrm{T}$ cell receptor (TCR) repertoire of the responder $\mathrm{CD} 8^{+} \mathrm{T}$ cells at these priming sites. We sought to determine if the repertoire of TCR V $\beta$ populations of cytotoxic T lymphocyte (CTL) induced following parenteral infection were the same as or distinct from those induced following oral infection. We infected $\mathrm{C} 3 \mathrm{HeB} / \mathrm{FeJ}$ mice orally or in the hind footpads with reovirus T1 $\mathrm{L}$ and assessed for changes in the $\mathrm{V} \beta$ repertoire among $\mathrm{CD} 8^{+} \mathrm{T}$ cells in Peyer's patches (PP), or popliteal lymph nodes (PLN), and spleens.

We noted a consistent expansion of $\mathrm{V}^{+} 6^{+} \mathrm{CD} 8^{+} \mathrm{T}$ cells in the spleens of animals infected 10 days previously by either route, although similar expansions were not consistently found in the local draining lymphoid tissues. We also assessed changes in the V $\beta$ repertoire of CTL lines that were re-stimulated with reovirus over a period of several weeks. We consistently observed expansion of the $\mathrm{V} \beta 6^{+} \mathrm{CD} 8^{+} \mathrm{T}$ cells, with occasional representation of other minor $\mathrm{V} \beta$ populations. Cell lines were sorted into enriched and depleted fractions of V $\beta$ populations of interest. Subsequent assessment of sorted fractions for anti-viral cytotoxicity by ${ }^{51} \mathrm{Cr}$ release assay confirmed a predominant role for $\mathrm{V} \beta 6^{+} \mathrm{CD} 8^{+} \mathrm{T}$ cells, and a variable minor role for $\mathrm{V} \beta 8^{+}$ $\mathrm{CD}^{+} \mathrm{T}$ cells following both oral and parenteral infection. In addition, we adoptively transferred enriched memory CTL populations from orally or parenterally infected $\mathrm{C} 3 \mathrm{H}$ donor mice into reovirus infected SCID recipient mice. We were able to retrieve large numbers of CTL from recipient mice that could mediate high levels of virus-specific cytotoxicity immediately ex vivo. $\mathrm{V} \beta$ analysis of the retrieved donor cells, cell lines, and cells removed from previously infected immunocompetent mice all tended to indicate preferential utilization of $\mathrm{V} \beta 6^{+} \mathrm{TCR}$. We subsequently assessed CDR $3 \beta$ length profiles $\mathrm{V} \beta 6^{+} \mathrm{CD} 8^{+}$cells from orally infected or parenterally infected ex vivo, after in vitro re-stimulation, or after adoptive transfer into infected SCID recipients. We observed a consistent expansion of one or more clones utilizing TCR $\beta$ chains of a single CDR3 $\beta$ length for orally and parenterally infected mice. Thus, we conclude that the route of infection with reovirus does not overtly affect the selection and priming of the repertoire of V $\beta$ TCR populations of CTL.
\end{abstract}




\section{Introduction}

Respiratory enteric orphan virus (Reovirus), serotype 1 strain Lang (T1L) is a nonenveloped, segmented dsRNA virus (Palmer and Martin, 1977) that is infectious in mice following oral and parenteral application, and elicits both humoral (London et al., 1987; Weinstein and Cebra, 1991) and cell-mediated immunity (Finberg et al., 1979; Letvin et al., 1981). After gaining access to the intestinal tissue via the M cells of the PP (Wolf et al., 1983), reovirus infection of immunocompetent mice causes a self-limited disease of enterocytes of the crypts of Lieberkuln adjacent to the PP of the distal ileum (Rubin et al., 1985). In response, virus-specific cytotoxic T lymphocytes (CTL) are induced within the PP (London et al., 1987). CTL may transit via efferent lymphatic vessels to the mesenteric lymph nodes and via the thoracic duct lymph and the systemic circulation to the spleen, peripheral lymph nodes (London et al., 1987), or intestinal mucosal sites such as the intestinal intraepithelial lymphocyte (IEL) compartment (London et al., 1989; Cuff et al., 1993; Chen et al., 1997). Parenteral infection with reovirus induces virus-specific CTL in the draining peripheral lymph nodes and spleen (Letvin et al., 1981). CTL induced following infection with reovirus are $\operatorname{CD} 8 \alpha \beta^{+} \mathrm{TCR} \alpha \beta^{+}$Thy $-1^{+} \mathrm{H}-2$ class I-restricted T cells (London et al., 1987). It has been demonstrated that reovirus-specific $\mathrm{CD}^{+}$CTL induced in the PP following enteric reovirus infection (London et al., 1987) and in the lung following reovirus bronchopneumonia (Thompson et al., 1996) express the unusual cell surface marker germinal center and T antigen (GCT), but it remains unknown what other differences may exist among the cytotoxic $\mathrm{T}$ lymphocyte populations that are induced following enteric or systemic reovirus infection, especially as regards their selection and specificity.

Recently, it has been demonstrated that murine reovirus bronchopneumonia following intratracheal instillation of reovirus T1L induces an unusual population of cytotoxic $\mathrm{CD} 4^{+}$ 
$\mathrm{CD} 8 \alpha \beta^{+} \mathrm{TCR} \alpha \beta^{+} \mathrm{T}$ cells (Periwal and Cebra, 1999), indicating that unique CTL populations might be induced by reovirus infection at distinct anatomic locations. Enteric reovirus T1L infection of $\mathrm{C} 3 \mathrm{HeB} / \mathrm{FeJ}$ mice has been shown to elicit $\mathrm{CD} 8 \alpha \beta^{+} \mathrm{CTL}$ populations expressing $\mathrm{V} \beta 12$ and $\mathrm{V} \beta 17$, with minor populations expressing $\mathrm{V} \beta 2, \mathrm{~V} \beta 7, \mathrm{~V} \beta 9$, and $\mathrm{V} \beta 14$ in the intestinal IEL compartment (Chen et al., 1997). These CTL populations were thought to be representative of the CTL response primed in the PP following oral infection with reovirus T1L (Cuff et al., 1993), although some may have been derived in situ given the uncertain ontogeny of the IEL compartment lymphocytes.

We infected $\mathrm{C} 3 \mathrm{H}$ mice orally or parenterally with reovirus, and assessed the $\mathrm{V} \beta$ repertoire of splenic virus-specific CTL populations induced to determine if the route of infection affected the selection of responder CTL populations. We also assessed the V $\beta$ repertoire of virus-specific pCTL primed in the PP, as well as those primed in the peripheral lymph nodes, to determine if the virus-specific CTL V $\beta$ populations recruited to the spleen following reovirus infection were representative of all the populations initially induced in the priming sites. Additionally, we adoptively transferred splenic and PP T cells from mice previously immunized orally or in the hind footpads with reovirus into reovirus-infected syngenic SCID mice to determine what CTL V $\beta$ populations would expand in the presence of continuous virus restimulation in vivo.

The humoral immune response to reovirus is influenced by micro-environmental or cellular factors at the anatomic site of infection (Weinstein and Cebra, 1991; Major and Cuff, 1996). It is not known, however, if intestinal reovirus infection results in a CTL response distinct from that following infection in the systemic periphery. Differences in the recruitment of naïve $\mathrm{T}$ cells, and local antigen processing and presentation within the PP as opposed to the peripheral 
lymph nodes may be reflected in the repertoire of CTL induced following oral or parenteral infection.

\section{Methods}

Animals: $7-8$ week old male $\mathrm{C} 3 \mathrm{HeB} / \mathrm{FeJ}$ and $\mathrm{C} 3 \mathrm{HSmnPkrc}$ severe combined immunodeficiency (SCID) mice were purchased from Jackson Laboratories (Bar Harbor, MA) and were housed in microisolator cages under specific pathogen free conditions. Mice were allowed to acclimate for one week, and were maintained on autoclaved food and water. Infected mice were housed in microisolator cages in a laminar flow cabinet in a separate room kept under negative pressure.

Virus: Third passage reovirus T1L stocks were grown in L929 cells. Cells were disrupted by sonication in a buffer of $0.01 \mathrm{M}$ Tris, $0.25 \mathrm{M} \mathrm{NaCl}, 0.01 \mathrm{M} ß$-mercaptoethanol, and $0.1 \%$ sodium deoxycholate ( $\mathrm{pH} 7.4$ ) and purified by freon extraction followed by step-wise $\mathrm{CsCl}$ gradient centrifugation as described (Smith et al., 1969). Infectious reovirus titers were assessed by plaque assay in L929 cells as described (Cuff et al. 1990).

Animal infections: Orally-infected mice received $3 \times 10^{7}$ plaque-forming units (pfu) of reovirus in $50 \mu \mathrm{l}$ of sterile borate-buffered saline and gelatin by gavage using a stainless steel feeding tube attached to a $1 \mathrm{ml}$ syringe. Hind footpad infected mice received $1.5 \times 10^{7} \mathrm{pfu}$ of reovirus in $50 \mu \mathrm{l}$ of sterile borate-buffered saline and gelatin in each hind footpad. SCID recipient mice received $3 \times 10^{7}$ pfu of reovirus in $100 \mu$ l total of sterile borate-buffered saline and gelatin by intraperitoneal injection.

Preparation and culture of splenic, PLN, and PP cells: Single cell suspensions of spleens and PLN cells were prepared by expressing tissues through sterile nylon mesh. Singlecell suspensions of PP were prepared by mechanical dissociation using sterile 18-gauge needles. 
Splenocytes were depleted of red blood cells by incubation in a hypotonic solution of $\mathrm{NH}_{4} \mathrm{Cl}$ and Tris-HCl. Splenocytes and PLN cells were washed 3 times in medium consisting of RPMI-1640 (Biowhittaker, Walkersville, MD) supplemented with 10\% FBS (Hyclone, Logan, VT), 2mM Lglutamine (Biowhittaker), 100U penicillin $/ \mathrm{ml}, 0.1 \mu \mathrm{g}$ streptomycin $/ \mathrm{ml}$ (Biowhittaker), and $50 \mu \mathrm{M}$ $\beta$-mercaptoethanol, (TCM). Cell viability was determined by trypan blue exclusion.

Generation of reovirus-specific $\mathbf{T}$ cell lines: CTL lines were generated by suspending cells in TCM to a concentration of $10^{7}$ cells $/ \mathrm{ml}$ prior to re-stimulation with reovirus T1L at a multiplicity of infection (MOI) of 1 for $1 \mathrm{hr}$. at $25^{\circ} \mathrm{C}$. Cells were subsequently re-suspended in TCM to a final concentration of $2 \times 10^{6}$ cells $/ \mathrm{ml}$ and incubated at $37^{\circ} \mathrm{C}$ in an atmosphere of $5 \%$ $\mathrm{CO}_{2}$ for the first week. Cultures were re-stimulated at weekly intervals using syngenic naïve splenic feeder cells previously pulsed for $1 \mathrm{hr}$. at $25^{\circ} \mathrm{C}$ with reovirus $\mathrm{T} 1 \mathrm{~L}$ and irradiated with $2000 \mathrm{rad}$ of gamma radiation.

Effector cells were re-stimulated with syngenic, RBC-depleted splenic feeder cells from naive mice that were resuspended in TCM to a concentration of $10^{7} / \mathrm{ml}$ and pulsed with $3^{\text {rd }}$ passage reovirus $\mathrm{T} 1 \mathrm{~L}$ at for $1 \mathrm{hr}$. at $25^{\circ} \mathrm{C}$. The feeder cells were then washed and irradiated with 2000 rad of gamma radiation. Irradiated feeder cells were added with effector splenocytes at a ratio of 5 feeders to each effector and brought to a final concentration of $2 \times 10^{6}$ cells $/ \mathrm{ml}$ in fresh TCM containing 5\% IL-2-containing conditioned media from Con A-stimulated rat splenocytes, and $5 \% 1 \mathrm{mM} \alpha$-methylmannoside. Cultures were incubated at $37^{\circ} \mathrm{C}$ in an atmosphere of $5 \%$ $\mathrm{CO}_{2}$.

Enrichment and Depletion of V $\beta$-expressing populations by indirect panning. Three days following in vitro restimulation with reovirus T1L, Ficoll-Hypaque gradient-enriched cells were treated with sterile PBS-diluted anti-V $\beta$ antibodies. Antibodies used were: MR9-4 (mouse 


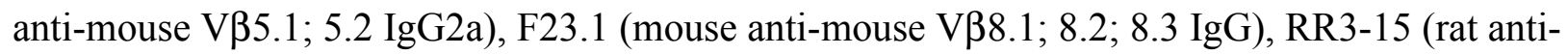

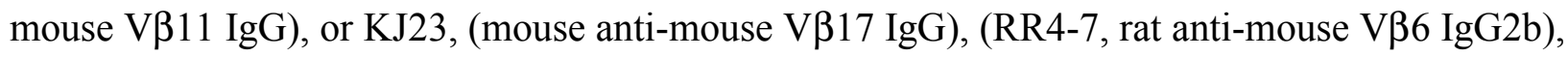

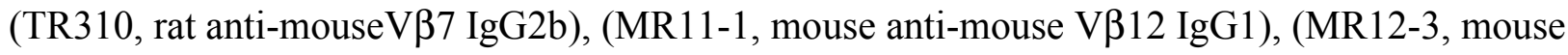

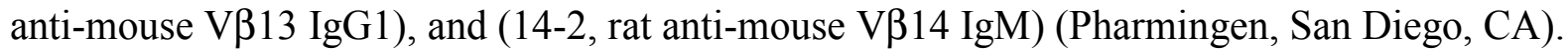
The cells were washed 3 times in PBS/5\% FCS, and resuspended in $4 \mathrm{ml}$ of PBS/5\% FCS. Half of the cells from each treatment group were panned by two successive incubations on sterile $16 \times$ $100 \mathrm{~mm}$ polystyrene bacteriology grade petri dishes (Falcon, Franklin Lakes, NJ) coated with $10 \mu \mathrm{g} / \mathrm{ml}$ goat anti-rat or goat anti-mouse IgG (Rockland, Gilbertsville, PA) in a $0.15 \mathrm{M}$ Tris buffer ( $\mathrm{pH}$ 9) for 45 minutes at $4^{\circ} \mathrm{C}$. Adherent cells were washed 3 times on the petri dishes with PBS/5\% FCS before being dislodged with a sterile rubber scrapper and cultured as above. Depleted and non-depleted cell cultures were assayed two days later for virus-specific cytotoxicity by ${ }^{51} \mathrm{Cr}$-release assay, and assessed for TCR expression by flow cytometry. Alternatively, depleted and enriched fractions were restimulated for a period of 1 week prior to analysis of and virus-specific cytotoxicity, using syngenic, irradiated, reovirus-pulsed thioglycollate-elicited peritoneal macrophages as feeder cells in a ratio of 1 feeder cell to 5 effector cells.

Adoptive transfer: RBC-depleted donor lymphocytes collected from mice that were infected 30 days previously were depleted of surface Ig-positive B cells by three successive incubations on sterile $16 \times 100 \mathrm{~mm}$ polystyrene bacteriology grade petri dishes (Falcon) coated with $100 \mu \mathrm{g} / \mathrm{ml}$ goat anti-mouse IgG, IgM and IgA antibody (Rockland) in a $0.15 \mathrm{M}$ Tris buffer (pH 9) for 45 minutes at $4^{\circ} \mathrm{C} .1 .5 \times 10^{7}$ non-adherent $\mathrm{T}$ cells were resuspended in $1 \mathrm{ml}$. of sterile PBS, and were injected intraperitoneally into reovirus-infected SCID recipient mice using a 20gauge needle. 
Depletion of $\mathrm{CD}^{+}$or $\mathrm{CD8}^{+}$cells: $\mathrm{CD}^{+}$or $\mathrm{CD}^{+} \mathrm{T}$ cells were depleted by treatment with anti-mouse CD4 IgM (RL172) or anti-mouse CD8 $\alpha$ IgM (3.155)-containing hybridoma culture supernatants, followed by the addition of Low-Tox rabbit complement (Cedarlane, Hornby, Ontario, Canada) to a final concentration of $10 \%$ by volume. The efficacy of complement-mediated depletion was determined by flow cytometry.

${ }^{51} \mathrm{Cr}$ release assay: Assays were performed as previously described (Cuff et al. 1998). Briefly, Ficoll-Hypaque gradient-purified effector CTLs were added in two-fold dilutions to duplicate or triplicate wells of $5 \times 10^{3}$ reovirus-infected or uninfected ${ }^{51} \mathrm{Cr}$-labeled $(200 \mu \mathrm{Ci}$; Dupont, Wilmington, DE) L929 target cells in a V-bottom 96-well plate (Nalgene, Rochester, NY) at effector: target cell ratios starting at 100 or 50:1. The assay plates were incubated for four hours at $37^{\circ} \mathrm{C}$ in an atmosphere of $5 \% \mathrm{CO}_{2}$. Specific lysis of reovirus infected targets for each effector: target ratio was calculated as described (London et al., 1987).

Flow cytometric analysis of effector cells: RBC-depleted mononuclear splenocytes, PP cells, and PLN cells from were stained with phycoerythrin (PE)-conjugated anti-CD8 $\alpha \mathrm{mAb}$ (CALTAG, Burlingame, CA), and fluorescein isothiocyanate (FITC)-conjugated mAbs to either CD4 or V $\beta 2,4,5,6,7,8.1 / 8.2,8.3,9,10,11,12,13,14,17$, (Pharmingen). In some experiments, cells were also stained with a biotinylated anti-V $33 \mathrm{mAb}$ (Pharmingen) followed by FITC-avidin (CALTAG). Percentages of cells staining positive for each marker were determined by analysis on a FACScan flow cytometer (Becton-Dickinson, Franklin Lakes, NJ), and results were further analyzed using WinMIDI 2.8 software (Joseph Trotter, Scripps Research Institute, La Jolla, CA).

CDR3 $\beta$ length profile analysis: Fragment analyses were performed by a modification of the methods originally reported by Pannetier et al., (1993). Briefly, single cell suspensions of 
purified $\mathrm{CD} 8^{+}$cells were prepared, and mRNA was isolated using the QIAGEN RNAeasy kit as per manufacturers instructions. Isolated mRNA was converted to cDNA and subsequently amplified using V $\beta 6$ or V $\beta 2$ primers and TAMRA-conjugated $C \beta$ primers (Integrated DNA Technologies, Coralville, IA) using sequences reported by Pannetier et al. using the QIAGEN RT-PCR kit (QIAGEN Inc., Valencia, CA) as per manufacturers instructions. Amplified products were analyzed using an ABI Prism model 377 automated sequencer (Applied Biosystems, Inc. Foster City, CA). CDR3 $\beta$ length distribution profiles were assessed by means of Genescan 3.0 software (Perkin Elmer, Norwalk, CT).

Statistical analysis: Means and standard deviations were calculated for each V $\beta$ population expressed among all groups of mice and tissues assessed by flow cytometry. Statistically significant differences in $\mathrm{V} \beta$ expression were determined by one - way analysis of variance followed by the Tukey test using Sigmastat 2.0 software (Jandel Scientific, Chicago, IL).

\section{Results}

\section{Reovirus $\mathrm{T} 1 \mathrm{~L}$ infection alters the $\mathrm{V} \beta$ repertoire of $\mathrm{CD8}^{+} \mathrm{T}$ cells in the spleen.}

The $\mathrm{V} \beta$ repertoire of $\mathrm{CD} 8^{+}$splenocytes from mice infected orally or in the hind footpads 10 days previously was assessed by flow cytometry (Figure 1). $\mathrm{CD} 8^{+}$splenocytes from footpadinfected mice showed a significantly elevated percentage of $\mathrm{V} 6^{+}$cells compared to orally infected mice $(p<0.05)$ and non-infected age-matched mice $(p<0.001)$ (Figure 1A). Additionally, orally infected mice had a significantly increased percentage of $\mathrm{V} \beta 9^{+} \mathrm{CD} 8^{+}$splenocytes compared to non-infected mice $(p=0.042)$. Absolute numbers of $\mathrm{CD} 8^{+}$splenocytes of each V $\beta$ population were calculated for orally infected, footpad infected, and non-infected mice (Figure 1B). Footpad infected mice had a significantly increased number of $\mathrm{V} \beta 6^{+}$and $\mathrm{V} \beta 7^{+} \mathrm{CD} 8^{+}$ 
splenocytes relative to non-infected mice ( $p=0.02$ and $p=0.022$, respectively). Orally infected mice also had elevated numbers of splenic $\mathrm{V} \beta 6^{+}$and $\mathrm{V} \beta 7^{+} \mathrm{CD} 8^{+}$cells compared to non-infected mice, but the differences were not significant ( $p=0.467$ and $p=0.404$, respectively).

In vitro re-stimulation with reovirus expands virus-specific $\mathrm{V} \beta$ populations of $\mathrm{CD} 8^{+} \mathrm{T}$ cells from the spleens of orally infected mice. Cultures of spleen cells from orally infected mice were assessed for changes in the $\mathrm{V} \beta$ repertoire of $\mathrm{CD} 8^{+} \mathrm{T}$ cells by flow cytometry and concomitantly assayed for enrichment of reovirus-specific CTLs by ${ }^{51} \mathrm{Cr}$ release assay. Cell lines consistently showed greatly increased percentages of $\mathrm{V} \beta 6^{+} \mathrm{CD} 8^{+} \mathrm{T}$ cells, with continued representation of minor $\mathrm{V} \beta 7^{+}$and $\mathrm{V} \beta 8.1,2^{+}$populations, while all other $\mathrm{CD} 8^{+} \mathrm{V} \beta$ populations decreased to very low levels after 5 weeks in vitro (Figure 2). In vitro re-stimulation with reovirus simultaneously enriched virus-specific CTLs, which mediated strong virus-specific cytotoxicity by ${ }^{51} \mathrm{Cr}$ release assay (Figure 3 ).

\section{In vitro re-stimulation with reovirus also expands virus-specific $\mathrm{V} 6^{+} \mathrm{CD8}^{+} \mathrm{T}$ cells from the spleens of hind footpad infected mice.}

Spleen cells from mice infected in the hind footpads with reovirus were similarly cultured and assessed for changes in $\mathrm{V} \beta$ repertoire of $\mathrm{CD} 8^{+}$cells and enrichment of virus-specific CTLs. As with cultures of splenocytes from orally infected mice, cultured splenocytes showed a greatly increased percentage of $\mathrm{VB} 6^{+} \mathrm{CD} 8^{+} \mathrm{T}$ cells, with representation of a few minor populations (Figure 4), concurrent with enriched virus-specific CTL activity (data not shown).

\section{Effect of oral and hind footpad infection with reovirus T1L on the $V \beta$ repertoire of $\mathrm{CD8}^{+}$cells in local lymphoid tissue.}

To determine if proliferative expansion of virus responsive $\mathrm{CD} 8^{+} \mathrm{T}$ cells in draining lymphoid tissues resulted in changes in the $\mathrm{V} \beta$ repertoire detectable by flow cytometry, mice were infected orally or in the hind footpads with reovirus. 7 days later, $\mathrm{CD} 8^{+} \mathrm{PP}$ lymphocytes 
from orally infected mice, and $\mathrm{CD} 8^{+}$PLN lymphocytes from footpad infected mice were stained with a panel of FITC-conjugated anti-V $\beta$ monoclonal antibodies, and compared to the V $\beta$ staining profiles of $\mathrm{CD} 8^{+} \mathrm{PP}$ or PLN lymphocytes from mock infected $\mathrm{C} 3 \mathrm{H}$ mice (Figure 5). No significant differences in the percentage expression of any $\mathrm{V} \beta$ population of $\mathrm{CD} 8^{+} \mathrm{T}$ cells were noted between mice infected by either route compared to mock infected controls.

\section{$V \beta$ repertoire of reovirus-specific CTL in PP and PLN.}

PP cells from orally infected mice or PLN cells from footpad-infected mice were cultured for a period of 2 weeks and assessed for changes in the V $\beta$ repertoire of $C D 8^{+} \mathrm{T}$ cells. PP and PLN cultures consistently showed increasing percentages of $\mathrm{V} \beta 6^{+}$cells (Figure 6), with concurrent enrichment of reovirus-specific CTL activity (Figure 7).

\section{$\mathrm{V} 6^{+} \mathrm{CD8}^{+}$cells mediate reovirus-specific cytotoxicity.}

Spleens from mice infected orally or in the hind footpads were expanded in culture for 3 weeks. Cultured cells were depleted of $\mathrm{CD} 4^{+} \mathrm{T}$ cells, the $\mathrm{CD} 8^{+}$cells were fractionated into $\mathrm{V} \beta 6^{+}$, $\mathrm{V} \beta 7^{+}$, or $\mathrm{V} \beta 8.1^{+}, 8.2^{+}$, and $8.3^{+}$depleted and enriched fractions by panning (Figure 8 ), and subsequently assessed for reovirus-specific cytotoxicity (Figure 9). The Vß6-enriched fractions of cultured splenocytes from both footpad infected mice (Figure 9B), and orally infected mice (Figure 9C) consistently mediated high levels of reovirus-specific cytotoxicity, whereas V $\beta 6$ depleted fractions showed diminished reovirus-specific killing (9A and C). By contrast, the V $\beta 7$ enriched fractions did not mediate reovirus-specific killing (9B). Interestingly, V $\beta 8$-enriched fractions did on rare occasion mediate a low level of killing (9B). No other $\mathrm{CD} 8^{+} \mathrm{T}$ cell $\mathrm{V} \beta$ populations in spleen cell lines from orally and footpad infected mice were shown to mediate reovirus-specific cytotoxicity, including $\mathrm{V} \beta 5, \mathrm{~V} \beta 9, \mathrm{~V} \beta 10, \mathrm{~V} \beta 11, \mathrm{~V} \beta 12, \mathrm{~V} \beta 13, \mathrm{~V} \beta 14$, or $\mathrm{V} \beta 17$ (not shown). 


\section{Adoptively transferred, reovirus-primed $\mathrm{CD8}^{+}$cells from immunocompetent donors expand in reovirus-infected SCID recipients.}

Splenocytes from $\mathrm{C} 3 \mathrm{H}$ mice infected orally or in the hind footpads with reovirus 30 days previously were depleted of B cells by panning on anti-murine Ig coated petri plates. $1.5 \times 10^{7}$ pooled cells $\left(4 \times 10^{6} \mathrm{CD} 8^{+}\right.$cells $)$were injected intraperitoneally into congenic SCID mice infected intraperitoneally with reovirus 4 days previously. Aliquots of donor cells were assessed for the $\mathrm{V} \beta$ repertoire of $\mathrm{CD} 8^{+}$cells to allow quantitation of the number of $\mathrm{CD} 8^{+}$cells of each $\mathrm{V} \beta$ population transferred to recipient mice. Recipient SCID mice were sacrificed at days 8,10 , and 12 post-transfer, and their splenocytes were stained for the $\mathrm{V} \beta$ expression (Figure 10). A dramatic expansion in the number of $\mathrm{V} \beta 6^{+} \mathrm{CD} 8^{+}$cells in the spleens of infected SCID recipients was observed for recipients of both orally infected and footpad infected donors. However, the number recovered from recipients of orally infected donors was significantly greater by day 12 post-transfer than the number recovered from recipients of footpad infected donors $(p<0.0144)$. The $\mathrm{V} B 7^{+} \mathrm{CD} 8^{+}$population was also increased in recipients of both donor groups, although spleens recovered from recipients of from orally infected donors had significantly more $\mathrm{V} \beta 7^{+}$ $\mathrm{CD} 8^{+}$cells than those of recipients of footpad infected donors at day 12 post-transfer $(p<0.0011)$. A similar outcome occurred for recovered V $\beta 8.1,2^{+} \mathrm{CD} 8^{+}$cells $(p<0.0089)$. Recipients of both donor groups also saw an expansion in the number of recovered $\mathrm{V} \beta 17^{+} \mathrm{CD} 8^{+}$over the course of the experiment. Reovirus infection of SCID recipient mice was essential in the recovery of donor cells and in the expansion of $\mathrm{V} \beta$ populations of $\mathrm{CD} 8^{+} \mathrm{T}$ cells, as immune donor cells transferred into non-infected SCID recipients showed no preference for expansion of any V $\beta$ population (not shown). 


\section{$\mathrm{CDB}^{+} \mathrm{T}$ cells recovered from the spleens of infected SCID recipients of immune splenocytes are enriched for virus-specific CTLs .}

Spleen cells recovered from infected SCID recipients of T cells from orally infected and footpad infected donor mice were assessed at days 8,10 , and 12 post-transfer for ex vivo cytotoxicity (Figure 11). Recovered spleen cells were depleted of $\mathrm{CD} 8^{+}$cells or $\mathrm{CD} 4^{+}$cells prior to ${ }^{51} \mathrm{Cr}$ release assay. Spleen cells recovered from infected SCID recipients of orally infected and footpad infected donors mediated strong ex vivo cytotoxicity. Depletion of $\mathrm{CD} 8^{+}$cells, but not $\mathrm{CD}^{+}$cells, ablated virus-specific killing. Reovirus infection of SCID recipient mice was essential in inducing CTL activity, as donor cells recovered from non-infected SCID recipients did not mediate ex vivo virus-specific cytotoxicity (not shown).

Adoptively transferred PP $\mathrm{CD} 8^{+}$lymphocytes from reovirus immune donors, but not those from naïve donors, expand in infected SCID recipients. $1 \times 10^{7}$ B cell-depleted PP lymphocytes from orally infected mice or naive mice were injected intraperitoneally into SCID mice infected with reovirus 4 days previously. Recipient SCID mice were sacrificed at day 10 post-transfer, and their splenocytes were stained for the $\mathrm{V} \beta$ repertoire of recovered $\mathrm{CD} 8^{+} \mathrm{T}$ cells (Figure 12). Consistent with the results for the adoptive transfer of immune spleen cells, a dramatic expansion in the number of $\mathrm{V} \beta 6^{+} \mathrm{CD} 8^{+}$cells in the spleens of infected SCID recipients was observed (Figure 12A). By contrast, $\mathrm{CD} 8^{+}$cells recovered from the spleens of infected SCID recipients of naive $\mathrm{CD} 8^{+}$lymphocytes showed no clear expansion of any $\mathrm{V} \beta$ population (Figure 12B).

\section{Adoptively transferred PP lymphocytes from reovirus immune donors, but not those from naïve donors, are enriched for virus-specific CTLs in reovirus-infected SCID recipients.}

Spleen cells recovered from infected SCID recipients of CD8 ${ }^{+}$PP lymphocytes from orally infected and naive donor mice were assessed at day 10 post-transfer for ex vivo cytotoxicity (Figure 13). Adoptively transferred $\mathrm{CD} 8^{+} \mathrm{PP}$ lymphocytes from immune donors 
recovered from the spleens of infected recipient SCID mice mediated strong reovirus-specific ex vivo cytotoxicity by ${ }^{51} \mathrm{Cr}$ release assay (Figure 13A). By contrast, $\mathrm{CD} 8^{+} \mathrm{PP}$ lymphocytes from naive donors recovered from infected SCID recipients did not mediate ex vivo reovirus-specific killing (Figure 13B).

In vitro re-stimulation results in the continued expansion of adoptively transferred immune $\mathrm{V}^{+} 6^{+} \mathrm{CD8}^{+} \mathrm{PP}$ lymphocytes recovered from the spleens of reovirus infected SCID recipients.

$\mathrm{CD} 8^{+} \mathrm{PP}$ lymphocytes from immune donor mice recovered from the spleens of reovirus infected SCID recipients at 10 days post-transfer were cultured for 3 weeks with reovirus pulsed PECs and Con-A conditioned media and assessed for changes in $\mathrm{V} \beta$ repertoire of $\mathrm{CD} 8^{+}$cells (Figure 14). Consistent with $\mathrm{CD} 8^{+}$splenic and PP lymphocytes from orally infected mice restimulated in vitro with reovirus, the percentage of $\mathrm{V} \beta 6^{+} \mathrm{CD} 8^{+}$cells increased with time.

\section{Effect of oral and hind footpad infection with reovirus T1L on the CDR3 $\beta$ length profile of $\mathrm{CD8}^{+}$cells in local lymphoid tissue and spleen.}

To determine if proliferative expansion of virus responsive $\mathrm{CD} 8^{+} \mathrm{T}$ cells in draining lymphoid tissues resulted in changes in the CDR3 $\beta$ length profile, mice were infected orally or in the hind footpads with reovirus. 10 days later, cells from spleens and the priming lymphoid tissues were depleted of $\mathrm{CD} 4^{+}$cells. $\mathrm{V} \beta 6^{+}$and $\mathrm{V} \beta 2^{+} \mathrm{CD} 8^{+} \mathrm{PLN}$ (Figure 15A) and splenic lymphocytes from footpad infected mice, and $\mathrm{V} \beta 6^{+}$and $\mathrm{V} \beta 2^{+} \mathrm{CD} 8^{+} \mathrm{PP}$ (Figure 15B) and splenic lymphocytes from orally infected mice were assessed for CDR3 $\beta$ length profile, and compared to the $\mathrm{CDR} 3 \beta$ length profiles of $\mathrm{V} \beta 6^{+}$and $\mathrm{V} \beta 2^{+} \mathrm{CD} 8^{+} \mathrm{PP}, \mathrm{PLN}$, or splenic lymphocytes from mock infected $\mathrm{C} 3 \mathrm{H}$ mice. $\mathrm{V} \beta 2$ was chosen as a control subpopulation, as $\mathrm{V} \beta 2^{+} \mathrm{CD} 8^{+}$cells consistently do not respond to reovirus $\mathrm{T} 1 \mathrm{~L}$ infection as determined by flow cytometry. No striking or consistent differences in the CDR3 $\beta$ length profile of PP, PLN or 
splenic $\mathrm{V} \beta 6^{+}$or $\mathrm{V} \beta 2^{+} \mathrm{CD} 8^{+} \mathrm{T}$ cells were noted between mice infected by either route compared to mock infected controls.

\section{Adoptively transferred, reovirus-primed $\mathrm{V} 6^{+}$, but not $\mathrm{V} \beta 2^{+}, \mathrm{CD8}^{+}$cells from reovirus-immune donors show altered CDR3 $\beta$ length profiles upon recovery from reovirus-infected SCID recipients.}

Spleen cells recovered from infected SCID recipients of splenic or PP T cells from orally infected donor mice, or splenic $\mathrm{T}$ cells from footpad infected donor mice depleted of $\mathrm{CD} 4^{+} \mathrm{T}$ cells were assessed at day 10 post-transfer for CDR3 $\beta$ length profiles among $\mathrm{V} \beta 6^{+}$or $\mathrm{V} \beta 2^{+}$ $\mathrm{CD} 8^{+}$cells. As previously shown, spleen cells recovered from infected SCID recipients of orally infected and footpad infected donors mediated strong ex vivo cytotoxicity (data not shown). $\mathrm{V} \beta 6^{+} \mathrm{CD}^{+}$cells recovered from reovirus-infected SCID recipients of cells from both orally infected and hind footpad infected donors showed dramatic and consistent alterations in the CDR3 $\beta$ length profile relative of the profiles for the input cells, with a predominant expansion of a subpopulation(s) bearing $\mathrm{V} \beta 6^{+}$TCR giving a CDR3 $\beta$ fragment length peak at 138bp (Figure $16)$.

\section{In vitro re-stimulation with reovirus alters the $\mathrm{CDR} 3 \beta$ length profiles $V \beta 6^{+} \mathrm{CD8}^{+} \mathrm{T}$ cells from the spleens of orally infected and footpad infected mice.}

Spleen cell lines from orally infected and parenterally infected mice were depleted of $\mathrm{CD}^{+}$cells and assessed for changes in the $\mathrm{CDR} 3 \beta$ length profile of $\mathrm{V} \beta 6^{+} \mathrm{CD} 8^{+}$or $\mathrm{V} \beta 2^{+} \mathrm{CD} 8^{+} \mathrm{T}$ cells. Cell lines from both orally infected and footpad infected mice consistently showed a uniform and dramatic alteration of the $\mathrm{CDR} 3 \beta$ length profile of $\mathrm{V} \beta 6^{+} \mathrm{CD} 8^{+} \mathrm{T}$ cells, with a predominant expansion of a clonal subpopulation(s) bearing $\mathrm{V} \beta 6^{+}$TCR giving a CDR3 $\beta$ length peak at 138bp (Figure 17). No consistent changes in the CDR3 $\beta$ length profile of $\mathrm{V} \beta 2^{+} \mathrm{CD} 8^{+}$ 
cells were noted. In vitro re-stimulation with reovirus simultaneously enriched virus-specific CTLs, which mediated strong virus-specific cytotoxicity (data not shown).

In vitro re-stimulation with reovirus further alters the CDR3 $\beta$ length profiles $V \beta 6^{+}$ $\mathrm{CD8}^{+} \mathrm{T}$ cells recovered from the spleens of infected SCID recipients of orally infected and footpad infected donors.

Purified $\mathrm{CD} 8^{+}$lymphocytes recovered at 10 days post-transfer from the spleens of reovirus infected SCID recipients of orally or footpad infected donors were re-stimulated for 3 weeks with reovirus, whereupon the cell lines were found to be nearly $100 \% \mathrm{~V} \beta 6^{+}$and $65 \%$ $\mathrm{V} \beta 6^{+}$, respectively, and mediated high levels of reovirus-specific cytotoxicity (data not shown). $\mathrm{CDR} 3 \beta$ length profile analysis of the $\mathrm{V} \beta 6^{+}$cells demonstrated a clear dominance of the $\mathrm{V} \beta 6^{+}$ cells by a clonal subpopulation(s) bearing TCR $\beta$ chains utilizing the single, uniform CDR3 $\beta$ length seen previously, regardless of the route of infection of the original donor mice (Figure $18)$.

\section{Discussion}

We sought to determine if the route of infection influenced the virus-specific CTL response. Specifically, we asked whether oral and footpad reovirus T1L infection of mice elicit different responder CTL populations utilizing distinct TCR V $\beta$ elements. There are many potential reasons why CTL induced following oral infection with reovirus could be different from those induced parenterally. During natural reovirus infection, the ingested virions undergo pancreatic chymotryptic proteolysis of the outer coat proteins in the duodenum to yield the infectious intermediate sub-viral particle (ISVP) (Bodkin et al., 1989). This processing within the lumen of the small intestine may generate antigenic determinants distinct from those generated in the systemic periphery. Additionally, cleaved viral antigens may be taken up by intestinal absorptive epithelial cells (Pang et al., 1981; So et al., 2000) which express a number 
of classical and non-classical antigen presentation and co-stimulatory molecules such as MHC class I, class II, CD1 and TL (mice) (Hershberg and Mayer, 2000; Matsumoto et al., 1992), CD1d (human), and the CD8 binding and activating ligand, gp180 (Campbell et al., 1999), and are thought to act as unconventional antigen presenting cells to lymphocytes of the intestinal mucosal (Kaiserlian and Etchart, 1999). Evidence also exists that lamina propria dendritic cells underlying the epithelium may sample luminal antigen directly (Kaiserlain and Etchart, 1999), or acquire antigen by from endocytosed, apoptotic intestinal epithelial cells (Huang et al., 2000), possibly acting in the priming of mucosal $\mathrm{T}$ cell populations (Muller et al., 2000). Thus, the generation of different antigenic determinants and the presence of distinct populations of antigen presenting cells could influence the priming of the CTL response.

10 days following oral or footpad infection of $\mathrm{C} 3 \mathrm{H}$ mice with reovirus $\mathrm{T} 1 \mathrm{~L}$, we observed a consistent expansion in the percentage and absolute number of splenic $\mathrm{V} \beta 6^{+} \mathrm{CD} 8^{+} \mathrm{T}$ cells compared to non-infected mice. However, while we also noted an increase in the percentage of $\mathrm{V} \beta 6^{+} \mathrm{CD} 8^{+}$cells in the PP 7 days following oral reovirus infection, we did not observe a similar increase of $\mathrm{V} 6^{+} \mathrm{CD} 8^{+} \mathrm{T}$ cells in the draining PLN of mice infected in the hind footpads. Nevertheless, the observed increase in splenic $\mathrm{V} \beta 6^{+} \mathrm{CD} 8^{+}$cells was consistently greater in footpad-infected mice than in orally infected mice (Figure 4). Uncertainty in the numbers of naïve $\mathrm{T}$ cells primed at each site, and the possible contribution of other potential draining lymphoid tissues such as the mesenteric lymph nodes or inguinal lymph nodes may tend to obscure the significance of these differences. However, the finding that the same V $\beta$ populations can be cultured from the spleen and the priming sites irrespective of the route of infection led us to analyze the spleen as a common repository of $\mathrm{CD} 8^{+}$memory cells derived from those $\mathrm{CD} 8^{+}$ cells induced in the priming tissues (Bousso et al., 1999). It is possible that differential CD8 ${ }^{+} \mathrm{T}$ 
cell migration and trafficking may be reflected in the disparity in observed $\mathrm{V} \beta 6^{+} \mathrm{CD} 8^{+} \mathrm{T}$ cells in draining lymphoid tissues and spleens. For instance, it has been observed that activated virusspecific naïve $\mathrm{T}$ cells primed in peripheral lymph nodes can deposit in the spleen, where subsequent rounds of proliferation dramatically increase the numbers of virus-specific $\mathrm{T}$ cells (Haanen et al., 1999). Thus, despite the fact that $\mathrm{V} \beta 6^{+} \mathrm{CD} 8^{+} \mathrm{T}$ cells seem not to be increased in the PLN of mice infected with reovirus T1L in the hind footpads, (although virus-specific $\mathrm{V} \beta 6^{+} \mathrm{CD} 8^{+} \mathrm{T}$ cells certainly are present as they can be expanded in vitro) activated $\mathrm{V} \beta 6^{+} \mathrm{CD} 8^{+} \mathrm{T}$ cells emigrating from the PLN that deposit in the spleen and subsequently proliferate may allow for our observation of elevated $\mathrm{V} \beta 6^{+} \mathrm{CD} 8^{+}$cell numbers in the spleen. This is likely also true for PP-derived ${\mathrm{V} \beta 6^{+}}^{+} \mathrm{CD} 8^{+} \mathrm{T}$ cells. However, the less dramatic increase noted in the spleens of orally infected mice, in light of the observed expansions of the percentage of $\mathrm{V} \beta 6^{+} \mathrm{CD} 8^{+} \mathrm{T}$ cells in the PP, may suggest that the gut-derived reovirus-specific CTL are less likely to deposit in the spleen, possibly instead trafficking preferentially to mucosal effector sites (Bode et al., 1999), perhaps by $\alpha_{4} \beta_{7}$-MadCAM-1 interaction (Farstad et al., 1996; Lefrancois et al., 1999).

Splenic, PP, and PLN cells consistently showed dramatically increased representation of $\mathrm{V} \beta 6^{+} \mathrm{CD} 8^{+} \mathrm{T}$ cells with continued restimulation in vitro. Other $\mathrm{CD} 8^{+} \mathrm{T}$ cell populations bearing other TCR V $\beta$ elements were also occasionally represented following several rounds of in vitro restimulation, albeit less consistently and to a less dramatic degree than the ubiquitous $\mathrm{V} \beta 6^{+}$ $\mathrm{CD}^{+} \mathrm{T}$ cells. These other $\mathrm{CD} 8^{+} \mathrm{V} \beta$-populations included cells bearing $\mathrm{V} \beta 8.1$ or V $\beta 8.2$, V $\beta 8.3$, $\mathrm{V} \beta 7, \mathrm{~V} \beta 14$, or $\mathrm{V} \beta 13$ in order of frequency of occurrence, and showed no association with the initial route of infection. However, only the $\mathrm{V} \beta 6^{+} \mathrm{CD} 8^{+}$cells consistently mediated virus-specific cytotoxicity, regardless of the initial route of infection. TheV $\beta 12^{+}$and $\mathrm{V} \beta 17^{+} \mathrm{CD} 8^{+} \mathrm{T}$ cell populations, found by Chen et al. (1997) to contain reovirus-specific cells within the IEL 
compartment of $\mathrm{C} 3 \mathrm{H}$ mice infected orally with reovirus $\mathrm{T} 1 \mathrm{~L}$, consistently could not be recovered following restimulation of splenocytes in vitro regardless of the route of infection. It should be remembered, however, that the intestinal IEL are an arcane population that may not be representative of conventional $\mathrm{T}$ cell populations.

It was possible that the different routes of infection yielded distinct reovirus-specific $\mathrm{CD} 8^{+} \mathrm{V} \beta$-populations that were not accurately represented by the CTL populations expanded in vitro. For instance, it was possible that culture conditions were causing the loss or underrepresentation of some virus-specific $\mathrm{CD}^{+} \mathrm{T}$ cell populations, as has been suggested in other culture systems (Arenz et al., 1997). Therefore, we sought to expand reovirus-specific CTL in vivo, in a model of chronic reovirus infection in the absence of humoral immunity. We adoptively transferred $\mathrm{B}$ cell-depleted splenic $\mathrm{T}$ cells from $\mathrm{C} 3 \mathrm{H}$ donors infected either orally or in the hind footpads with reovirus T1L, into reovirus-infected C3H SCID mice to observe the expansion of reovirus-specific $\mathrm{CD} 8^{+} \mathrm{T}$ cells. We reasoned that immune cells must expand in reovirus infected SCID mice, as previous reports indicated that unfractionated immune Peyer's patch cells adoptively transferred into SCID recipients were able to clear reovirus infection (George et al., 1990). Immune cells were transferred one month post-infection with reovirus to allow for the redistribution of reovirus-specific Ig-producing plasma cells from the spleen to effector sites such as the bone marrow (Slifka et al., 1995), as direct panning for surface Igpositive splenic B cells would not otherwise allow the effective depletion surface Ig-negative plasma cells from the transferred donor splenocytes. Transferred splenocytes and their progeny were recovered from the spleens of reovirus infected SCID mice at various time points in the second week post-transfer, and demonstrated high levels of virus-specific cytotoxicity immediately ex vivo. This cytotoxicity was completely abrogated by complement and 
monoclonal antibody mediated depletion of $\mathrm{CD} 8^{+}$cells but not $\mathrm{CD} 4^{+}$cells, thus demonstrating that recovered $\mathrm{CD} 8^{+} \mathrm{T}$ cells were responsible for the observed virus-specific cytotoxicity, and not $\mathrm{CD} 4^{+} \mathrm{CTL}$ or activated NK cells (Taterka et al., 1995). $\mathrm{CD} 4^{+}$cells were not depleted from the initial bolus of transferred donor cells to provide virus-specific $\mathrm{T}$ helper cell support, as we were concerned that massive stimulation of $\mathrm{CD} 8^{+} \mathrm{T}$ cells without $\mathrm{CD} 4^{+} \mathrm{T}$ cell support would lead to clonal exhaustion, as has been reported in murine LCMV infection (Battegay et al., 1994; Matloubian et al., 1994). Interestingly, in other adoptive transfer systems the relative ratio of $\mathrm{CD}^{+}$to $\mathrm{CD} 8^{+} \mathrm{T}$ cells transferred into $\mathrm{T}$ cell deficient mice has been shown to be tightly regulated (Rocha et al., 1989). However, we observed a dramatic and rapid inversion of the CD4/CD8 ratios of recovered donor cells, a result which closely agrees with the findings of Zimmermann and Pircher (1999) and Zimmermann et al., (1999) in which immune T cells from mice previously infected with vaccinia virus, vesicular stomatitis virus, or lymphocytic choriomeningitis virus (LCMV), that were subsequently transferred into congenic recipient mice infected with the homologous virus showed a rapid expansion of donor $\mathrm{CD} 8^{+} \mathrm{T}$ cells and an inversion of the donor $\mathrm{CD} 4 / \mathrm{CD} 8 \mathrm{~T}$ cell ratio by the second week post-transfer. The rapid proliferation of $\mathrm{CD}^{+} \mathrm{T}$ cells in our transfer experiments is also due mainly to the activation of memory CTL, as T cells recovered from the spleens of infected SCID recipients of PP T cells from naïve donors could not mediate reovirus-specific cytotoxicity ex vivo. Additionally, any non-specific proliferation of donor $\mathrm{CD} 8^{+} \mathrm{T}$ cells in the recipient to fill the otherwise empty $\mathrm{T}$ cell compartment (Rudolphi et al., 1991), a phenomenon called 'blind homeostasis' (Cole, 1995), did not result in same the $\mathrm{V} \beta$ repertoire distribution we observed for infected SCID recipients of immune $\mathrm{T}$ cells, as $\mathrm{CD} 8^{+}$spleen cells recovered from the infected SCID recipients of naïve PP 
cells, as well as $\mathrm{CD} 8^{+}$spleen cells recovered from non-infected SCID recipients of immune donor splenocytes, showed no preferential expansion of any $\mathrm{V} \beta$ population.

$\mathrm{V} \beta$ analysis of the recovered $\mathrm{CD} 8^{+}$cells from infected SCID recipients of both orally and hind-footpad infected donor mice demonstrated a dramatic and consistent expansion in the percentage and number of $\mathrm{V} \beta 6^{+} \mathrm{T}$ cells, with other minor expansions in some other populations, notably those utilizing $\mathrm{V} \beta 7$ or $\mathrm{V} \beta 8$. The $\mathrm{V} \beta$ repertoire profile recovered $\mathrm{CD} 8^{+}$cells did not, however, demonstrate any overt differences in responder $\mathrm{CD} 8^{+} \mathrm{T}$ cell $\mathrm{V} \beta$ subpopulations dependent on the route of infection of the donor mice, and overall the results closely agreed with our previous analyses of cultured $\mathrm{CD} 8^{+}$cells from orally and footpad infected animals. Further studies, using enriched $\mathrm{T}$ cells from the $\mathrm{PP}$ of immune donor $\mathrm{C} 3 \mathrm{H}$ mice infected orally with reovirus T1L one month previously transferred into reovirus infected C3H SCID mice also resulted in the expansion in vivo of $\mathrm{V} \beta 6^{+} \mathrm{CD} 8^{+} \mathrm{T}$ cells, indicating that the reovirus-specific $\mathrm{CD} 8^{+}$ $\mathrm{T}$ cells recovered from the spleens of recipient SCID mice reflected those reovirus-specific cells from the initial priming tissues. Thus, we conclude that the predominant CTL response to both oral and footpad infection of $\mathrm{C} 3 \mathrm{H}$ mice with reovirus $\mathrm{T} 1 \mathrm{~L}$ is of $\mathrm{V} \beta 6^{+} \mathrm{CD} 8^{+} \mathrm{T}$ cells. We further conclude that the recovered splenic populations are reflective of the $\mathrm{T}$ cell populations induced to respond at the initial priming sites, and therefore that the anatomic site of infection does not affect the selection of responder $\mathrm{CD} 8^{+} \mathrm{T}$ cell populations.

There are indications, however, that reovirus infection in distinct mucosal compartments, may prime unique CTL populations. For example, murine bronchopneumonia following intratracheal instillation with reovirus T1L primes CTL responses in the draining tracheobronchial and mediastinal lymph nodes, including unique $\mathrm{CD} 4^{+} \mathrm{CD} 8 \alpha \beta^{+} \mathrm{TCR} \alpha \beta^{+} \mathrm{CTL}$ (Periwal and Cebra, 1999). Additionally, Chen et al. (1997) determined that enteric reovirus T1L 
infection of $\mathrm{C} 3 \mathrm{HeB} / \mathrm{FeJ}$ mice induced reovirus-specific $\mathrm{CTL}$ in the intestinal IEL compartment that were $\mathrm{V} \beta 12^{+}$, or $\mathrm{V} \beta 17^{+}$, with other apparently virus-responsive $\mathrm{CD} 8^{+}$populations expressing either $\mathrm{V} \beta 2$, V $\beta$ 7, V $\beta$ 9, or $\mathrm{V} \beta 14$. We demonstrated that the CTL V $\beta$ populations recovered from the PP and spleen or the PLN and spleen following enteric and systemic infection of C3HeB/FeJ mice, respectively, were essentially the same, yet are distinct from those Chen et al. (1997) expanded in culture out of the IEL compartment. However, although evidence exists that reovirus-specific CTL recovered from the IEL compartment are originally primed in the $\mathrm{PP}$, the IEL compartment is largely populated by unconventional $\mathrm{CD} 8^{+} \mathrm{T}$ cell of uncertain ontogeny and function, which may not be representative of conventional $\mathrm{T}$ cells of the peripheral lymphoid tissues. Indeed, the presence of unusual lymphoid structures such as cryptopatches (Saito et al., 1998; Ishikawa et al., 1999), and lymphocyte filled villi (Moghaddami et al., 1998), postulated to provide intestinal sites of extrathymically-derived IEL, suggests that T cells produced and primed in situ may provide a source of virus-specific CTL in addition to those derived from the PP.

In any event, whether or not enteric reovirus infection induces minor populations of CTL of unconventional ontogeny, the CTL response was overwhelmingly dominated by $\mathrm{V} \beta 6^{+} \mathrm{CD} 8^{+}$ that, at the level of flow cytometric analysis, was not different from the CTL induced following parenteral footpad infection. However, distinct clonal responses might occur within the broad ${\mathrm{V} \beta 6^{+}}^{+}$response dependent on the anatomic site of infection. A more detailed characterization of the responder $\mathrm{V} \beta 6^{+} \mathrm{CD} 8^{+} \mathrm{T}$ cells in the spleens and priming lymphoid tissues was performed by CDR3 $\beta$ length profile analysis. While no difference ex vivo was found in the CDR3 $\beta$ length profile of $\mathrm{V} \beta 6^{+} \mathrm{CD}^{+}$from spleens and priming tissues relative to non-infected controls, restimulation with reovirus in vitro, and adoptive transfer of immune cells into infected SCID 
recipients yielded $\mathrm{V} \beta 6^{+} \mathrm{CD} 8^{+} \mathrm{CTL}$ with a consistent expansion of one or a few clones utilizing TCR $\beta$ chains of the same length. This CDR3 $\beta$ length profile was uniformly found in $\mathrm{V} \beta 6^{+}$ $\mathrm{CD} 8^{+} \mathrm{CTL}$ initially primed by either oral or parenteral infection, further suggesting that the route of infection does not select for the priming of different populations of responder $\mathrm{CD} 8^{+}$cells. These findings imply that the antigen processing and presenting machinery utilized by APC at the draining lymphoid tissue of different anatomic sites is conserved, indicating that perhaps viral antigen processing and epitope presentation events do not differ between the intestinal immune system and that of the systemic periphery. Thus, the cellular immune system seems to utilize similar strategies and cell populations to control infection within the intestine and the systemic periphery. 

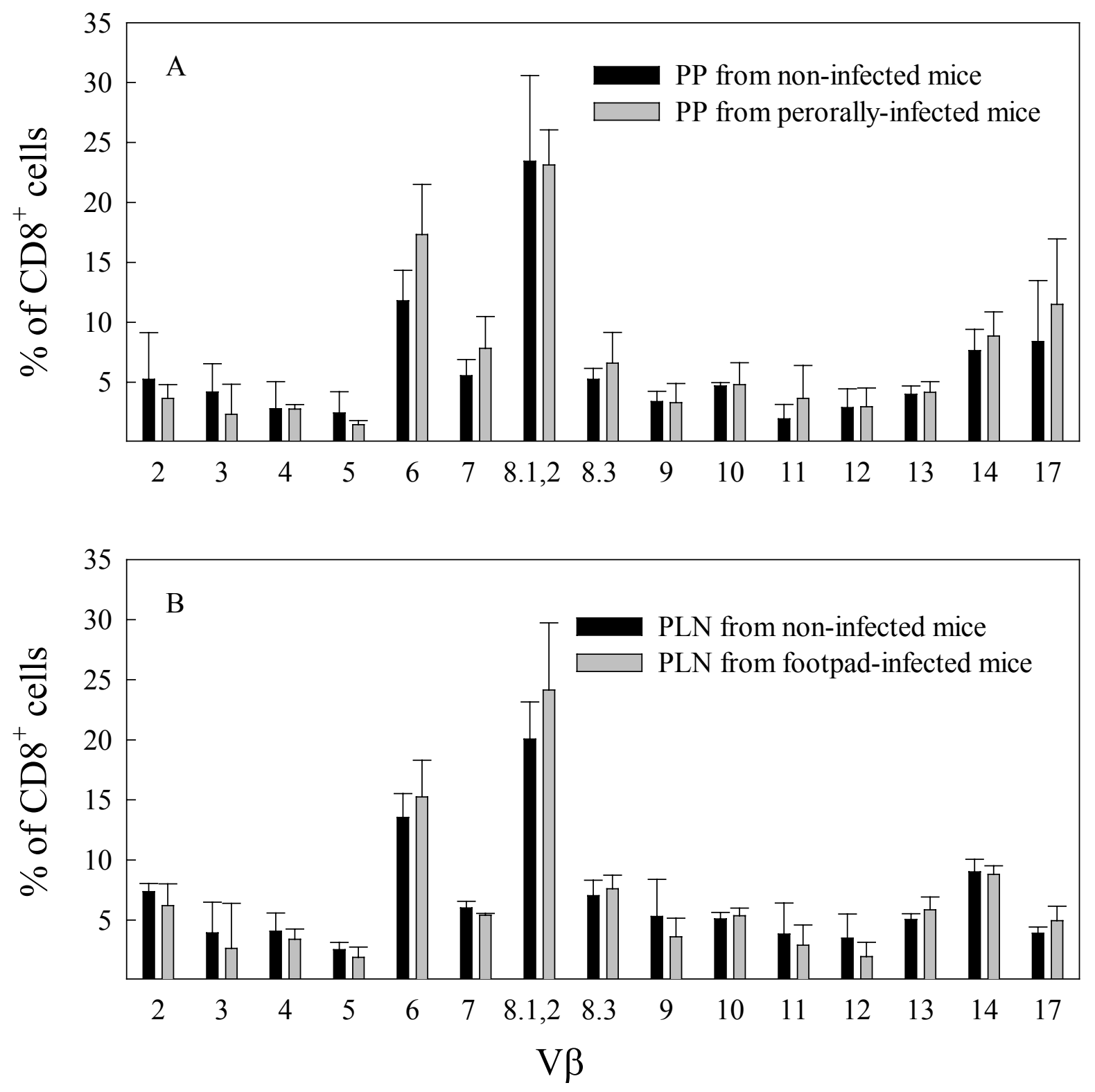
Figure 1. V $\beta$ repertoire of $\mathrm{CD8}+\mathrm{T}$ cells from the Peyer's patches of $\mathrm{C} 3 \mathrm{H}$ mice infected perorally and the popliteal lymph nodes of mice infected in the hind footpads with reovirus 7 days previously (A and B respectively), compared to Peyer's patch and popliteal I Data are expressed as mean percentage \pm standard deviations. 

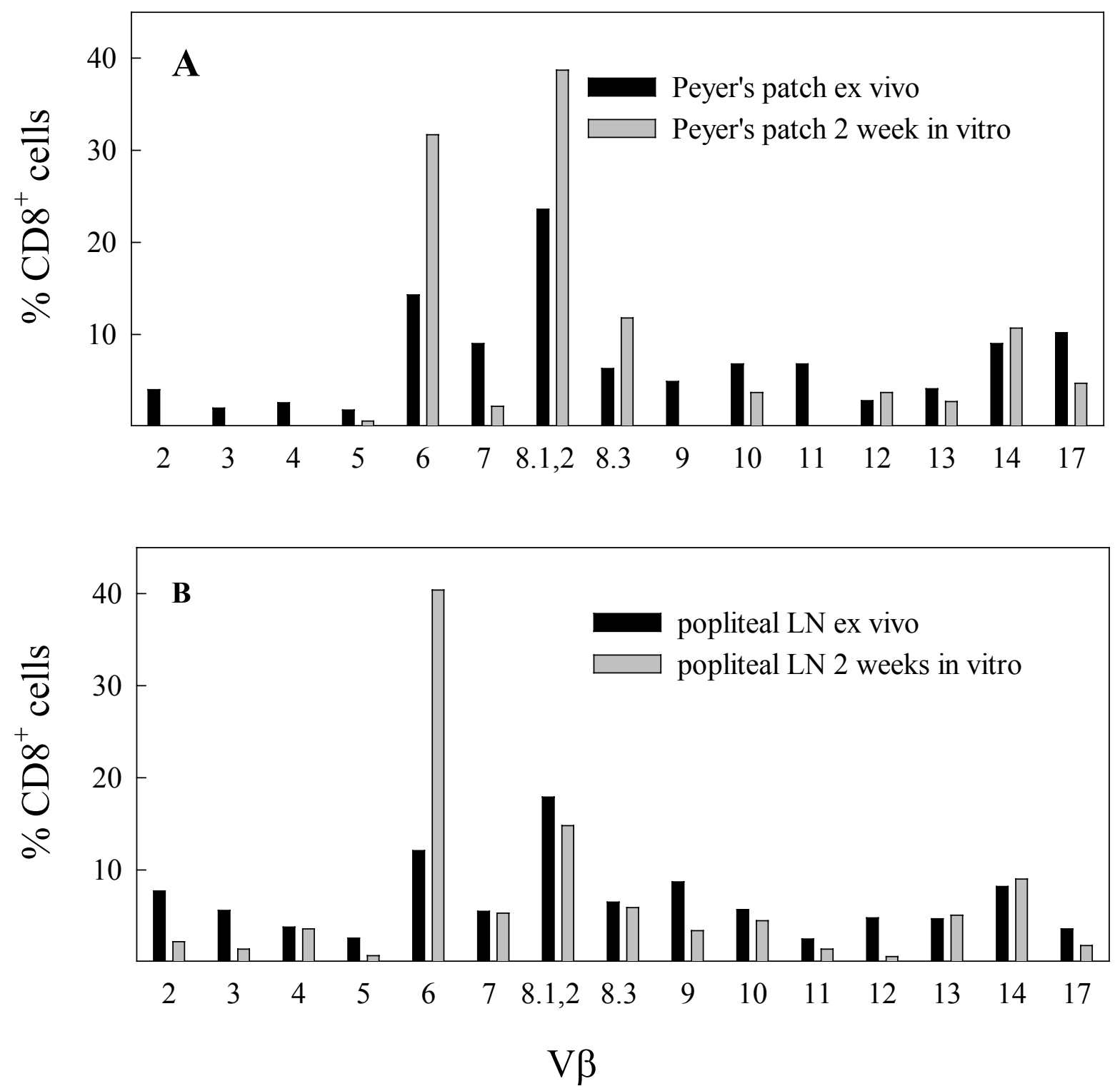
Figure 2. V $\beta$ repertoire of representative cultures of $\mathrm{CD8}+$ Peyer's patches from $\mathrm{C} 3 \mathrm{H}$ mice infected perorally and popliteal lymph nodes from mice infected in the hind footpads with reovirus (A and $B$ respectively). Shown are the percentages of $\mathrm{CD8}+$ cells staining positive with a panel of FITC-anti-V $\beta$ antibodies immediately ex vivo and after 2 weeks of in vitro

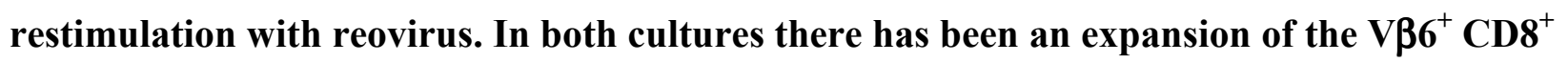
subpopulation, with $\mathrm{V} \beta 8.1,2^{+}$and $\mathrm{V} \beta 8.3^{+}$also showing increased representation in Peyer's patch cultures. Data is show as mean percentage expression. 


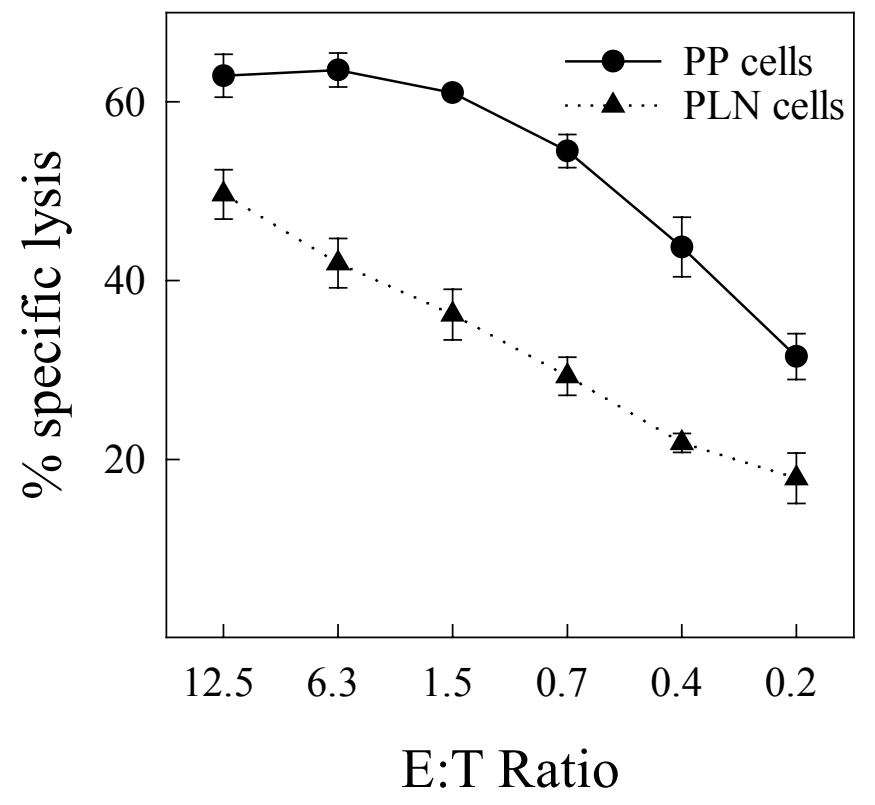


Figure 3. $51 \mathrm{Cr}$ release assay of Peyer's patch and popliteal lymph node cells derived from $\mathrm{C} 3 \mathrm{H}$ mice infected perorally or in the hind footpads and restimulated with reovirus in vitro for a period of 2 weeks. Data is given as mean percent specific lysis \pm standard deviation. 

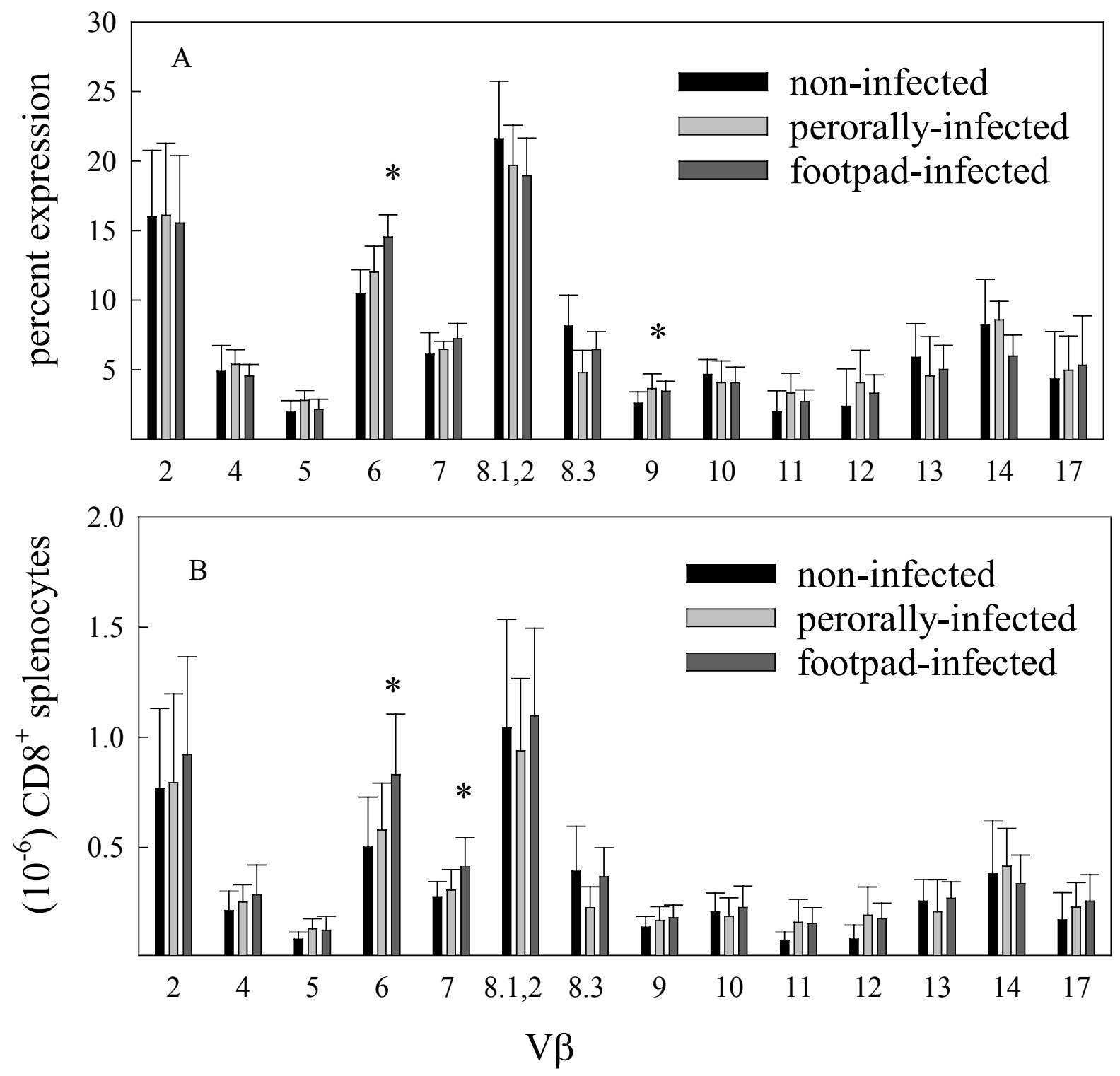
Figure 4. Ten days following peroral or hind footpad infection of $\mathrm{C} 3 \mathrm{H}$ mice with reovirus, splenocytes were assessed by flow cytometry for $\mathrm{V} \beta$ repertoire expression among CD8+ $T$ cells. (A.) The percentage of $\mathrm{CD8}+$ cells staining positive with each anti-V $\beta$ antibody were determined for infected and non-infected control mice. Data are given as mean percentage \pm standard deviation. For all three groups, $n=9$.

*Significant differences by one way ANOVA followed by the Tukey test. Footpad infected mice have an increased percentage of $\mathrm{V}^{+} 6^{+} \mathrm{CD}^{+}$cells compared to perorally infected and noninfected mice $(p<0.05$ and $p<0.001$, respectively). Perorally infected mice show increased expression of $\mathrm{V} \beta 9^{+} \mathrm{CD} 8^{+}$cells compared to non-infected $(p=0.042)$.

(B.) The absolute numbers of $\mathrm{CD}^{+}$expressing each V $\beta$ were calculated. Data are given as (means \pm standard deviations) $\times 10^{-6} \mathrm{CD}^{+}$cells.

Significant differences as determined by one-way ANOVA followed by the Tukey test. Footpadinfected mice have a greater number of $\mathrm{V} 6^{+}$and $\mathrm{V} \beta 7^{+} \mathrm{CD}^{+}$splenocytes compared to noninfected controls $(p=0.02$ and $p=0.022$, respectively). 

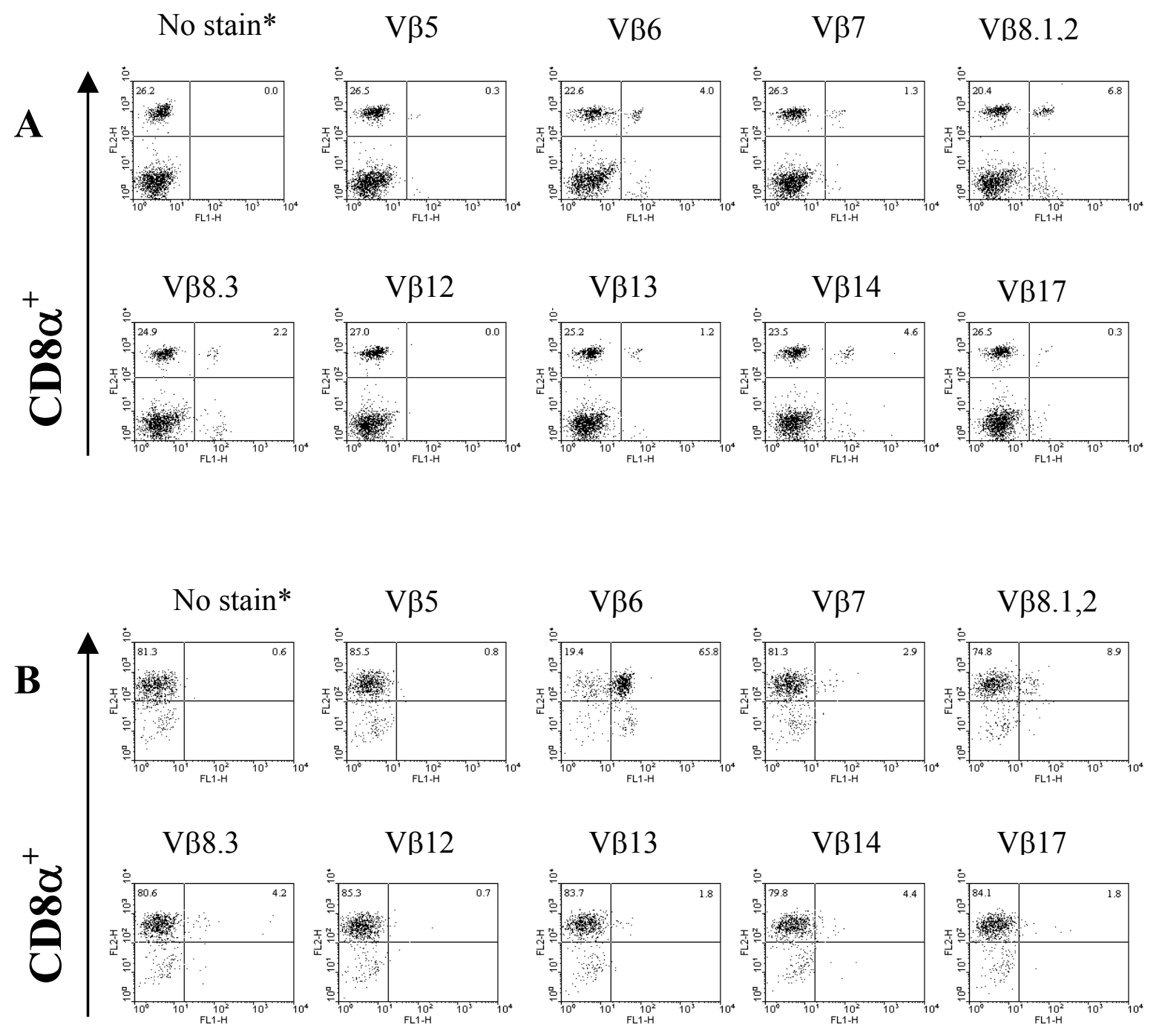
Figure 5. V $\beta$ repertoire of cultured CD8+ splenocytes from $\mathrm{C} 3 \mathrm{H}$ mice infected perorally with reovirus. Shown are selected VB staining profiles of CD8+ splenocytes (A) and after 5 weeks in culture (B). Restimulation in vitro with reovirus during culture has selectively expanded the VB6+CD8+subpopulation, with conspicuous representation of the VB7+,

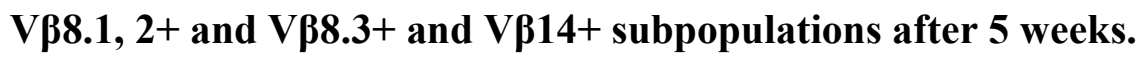

* No stain panels represent cells stained with PE-anti-CD8 $\alpha^{+}$alone. The percentage of cells in the upper right quadrant are subtracted from the percentage of $\mathrm{CD}^{+}$cells staining positive for each FITC-V $\beta$ antibody to correct for false-positives. 


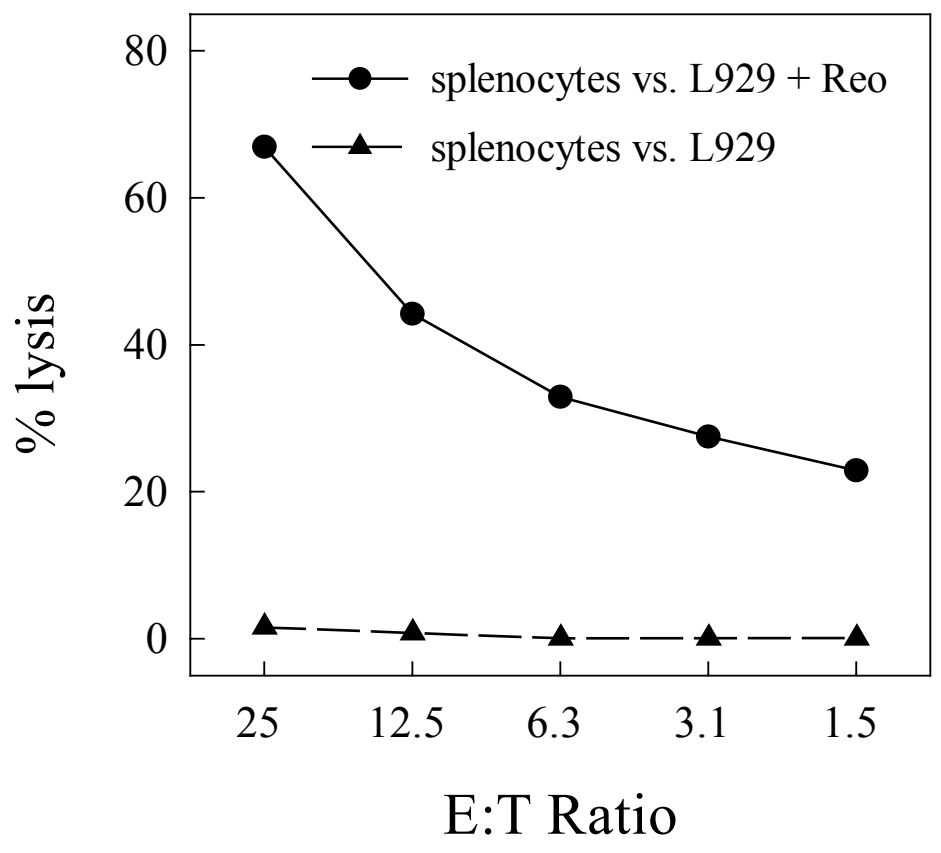


Figure 6. $51 \mathrm{Cr}$ release assay of splenocytes derived from $\mathrm{C} 3 \mathrm{H}$ mice previously infected perorally with reovirus. Splenocytes were restimulated in vitro for a period of 5 weeks prior to analysis. Data is given as mean lysis. 

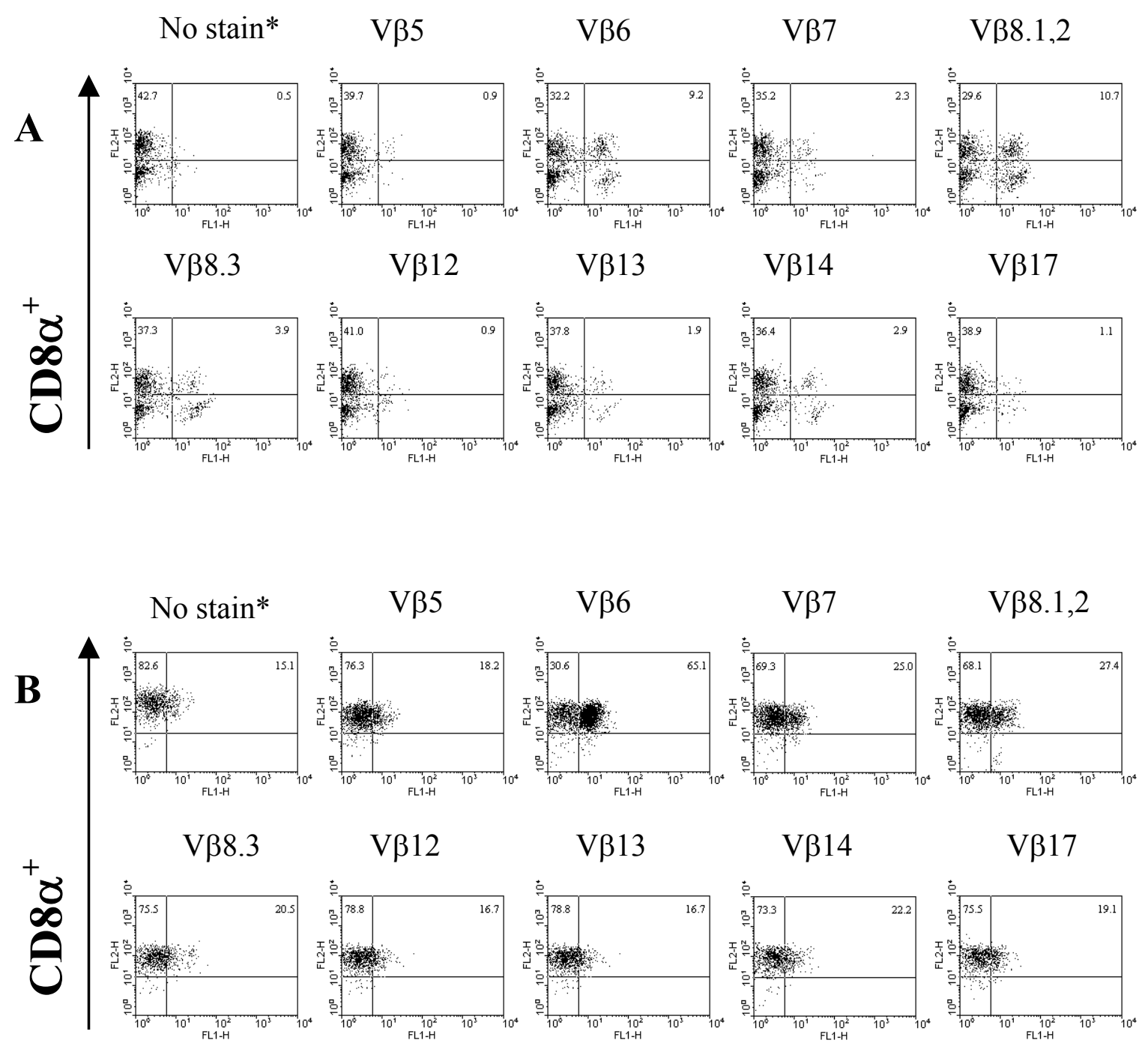
Figure 7. Figure 7. Vß repertoire of CD8+ splenocytes from $\mathrm{C} 3 \mathrm{H}$ mice infected with reovirus in the hind footpads. Shown are selected VB staining profiles of CD8+ splenocytes after 2 weeks of culture (A) and after 5 weeks of culture (B). Re-stimulation with reovirus in vitro has selectively expanded the $\mathrm{V} 6^{+} \mathrm{CD8}^{+}$subpopulation, and to a lesser extent the

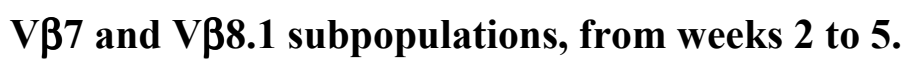

*No stain panels represent cells stained with PE-anti-CD8 $\alpha^{+}$alone. The percentage of cells in the upper right quadrant are subtracted from the percentage of $\mathrm{CD}^{+}$cells staining positive for each FITC-V $\beta$ antibody to correct for false-positives. 

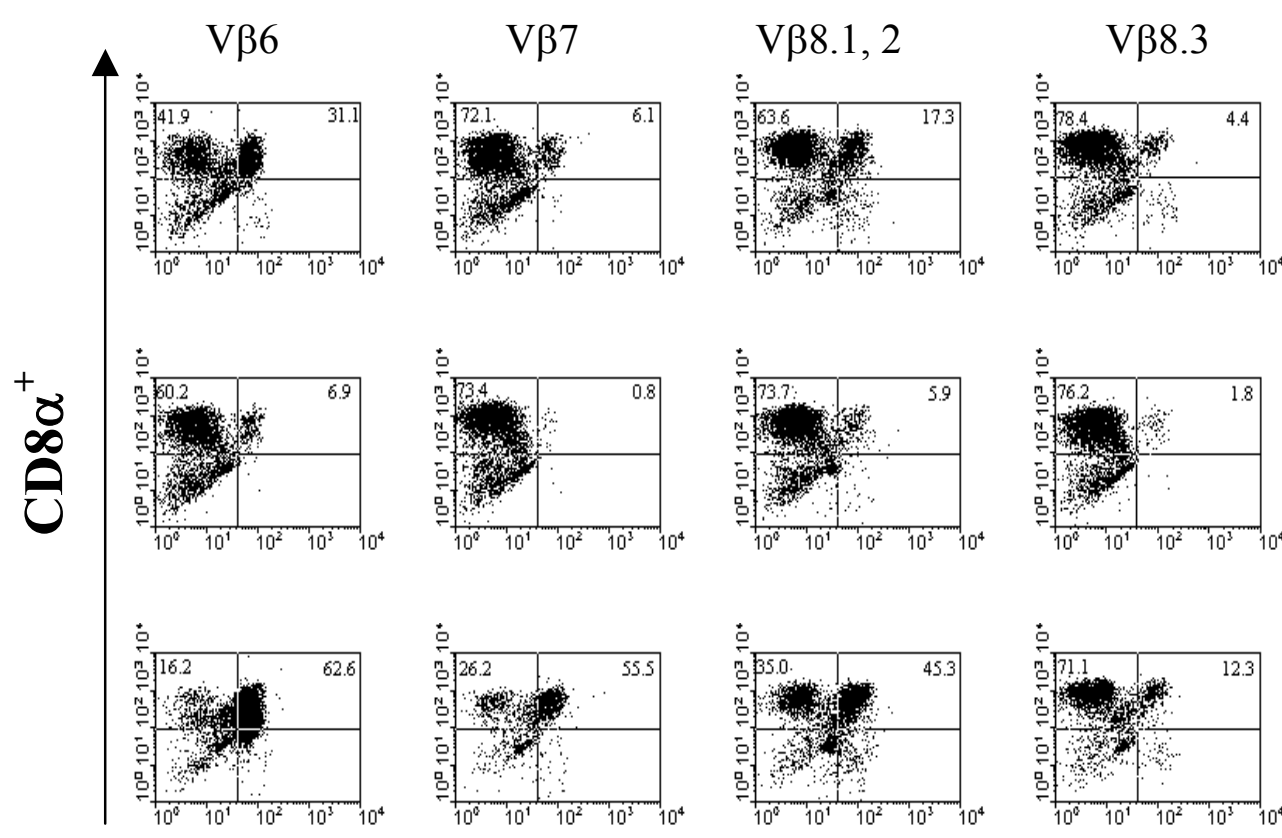

not sorted
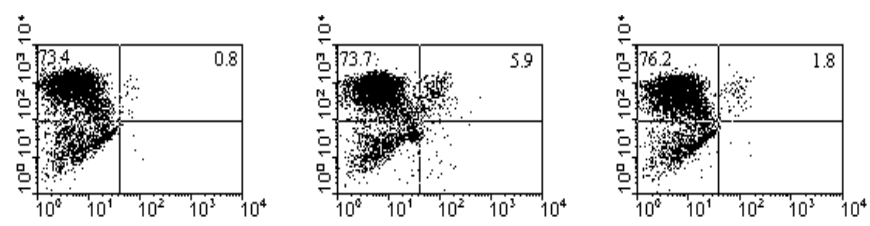

depleted
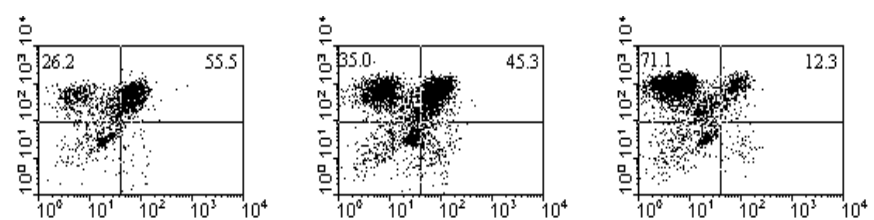

enriched 
Figure 8. Flow cytometric analysis of the V $\beta 6, V \beta 7$, and $V \beta 8$ expression of fractions of sorted CD8+ splenocytes from footpad-infected C3H mice. Splenocytes were cultured for 3 weeks and complement depleted of CD4+ cells as described in methods. CD8+ cells were then sorted into $\mathrm{V} \beta 6^{+}, \mathrm{V} \beta 7^{+}$, and $\mathrm{V} \beta 8^{+}$enriched and depleted fractions by indirect panning. 


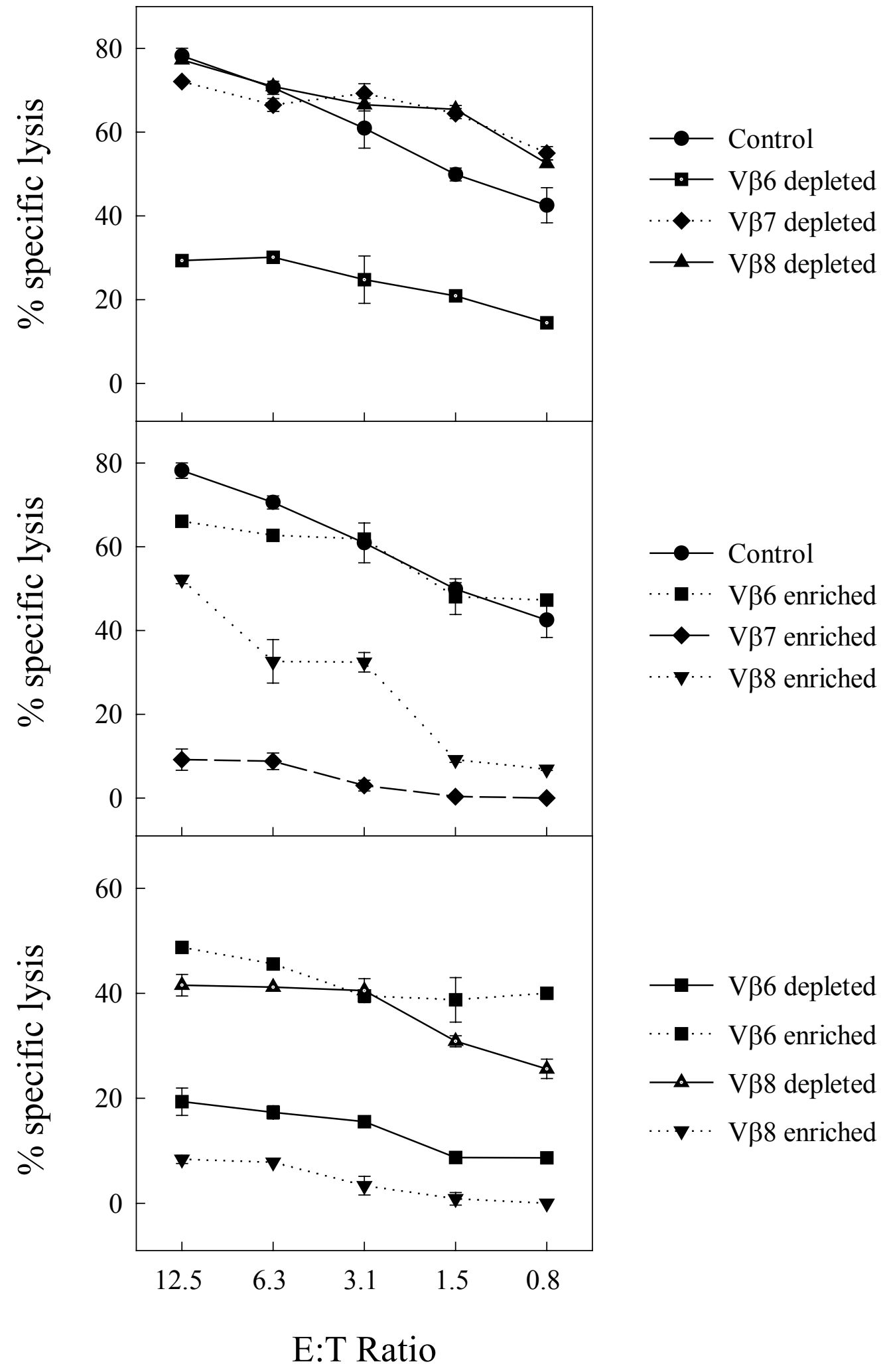


Figure 9. Representative 51Cr release assays of virus-specific cytotoxicity mediated by selected V $\beta$ subpopulations of cultured CD8+ splenocytes from C3H mice previously infected in the hind footpads or perorally with reovirus. Splenocytes from footpad and perorally infected mice were cultured for 3 weeks and then complement depleted of CD4 ${ }^{+}$ cells as detailed in methods. $\mathrm{CD8}^{+}$cells from footpad infected mice were then incubated with monoclonal antibodies specific for either $V \beta 6, V \beta 7$, or $V \beta 8.1,2$, and 3. Incubated cells were then separated by indirect panning into enriched and depleted fractions of the V $\beta$ subpopulation of interest prior to analysis of anti-viral cytotoxicity by ${ }^{51} \mathrm{Cr}$ release assay, as detailed in methods ( $A$ and $B$ are depleted and enriched fractions, respectively). $\mathrm{CD8}^{+}$ splenocytes of perorally infected mice were similarly sorted and assessed for anti-viral cytotoxicity (C). 

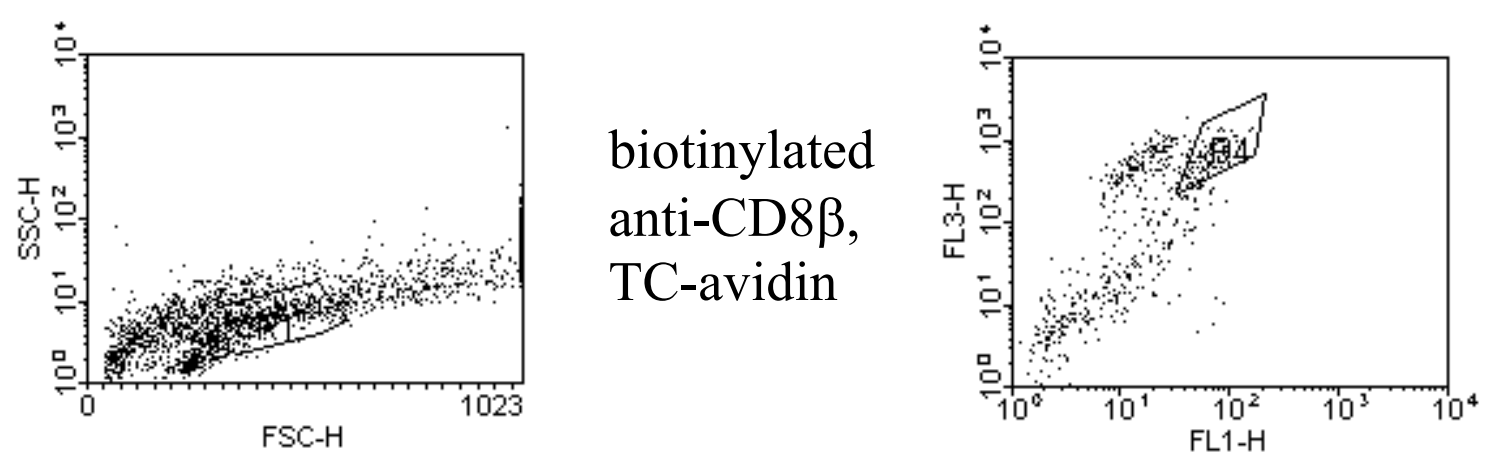

FITC-antiV $\beta 6$
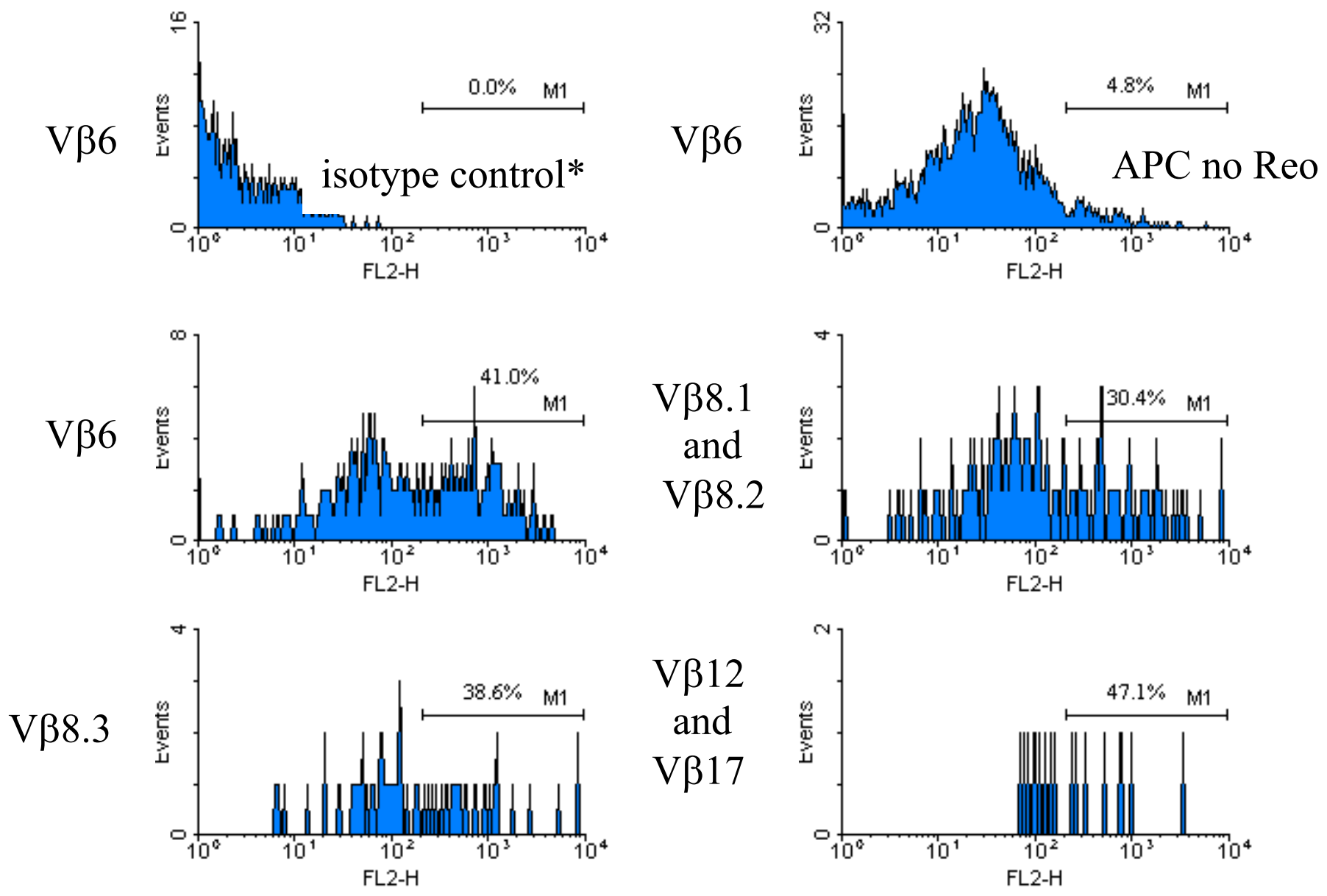
Figure 10. Intracellular interferon- $\gamma$ staining of a CD8+ splenocytes from perorally infected C3H mice after 3 weeks of reovirus restimulation in vitro. $5.0 \square \mathrm{x} 106$ cell fractions were incubated for 6 hours in the presence of 2.5x106 PECs either pulse or unpulsed with reovirus as described in methods. Brefeldin A was added for the last 5 hours of culture. Cells were stained first with anti-Fc $\gamma R$, then with antibodies to CD8 $\beta$ and select V $\beta$ subpopulations. $C D 8 \beta^{+} \mathrm{V} \beta 6^{+}, \mathrm{V} \beta 8.1,2^{+}, \mathrm{V} \beta 8.3^{+}$, or $\mathrm{V} \beta 12^{+}$and $\mathrm{V} \beta 17^{+}$cells were permeabilized and assessed for the intracellular accumulation of interferon- $\gamma$ with PE-antiIFN- $\gamma$ antibody.

*Isotype control antibody, PE-anti-rat IgG2b, added to splenocytes co-cultured with reoviruspulsed APC. 

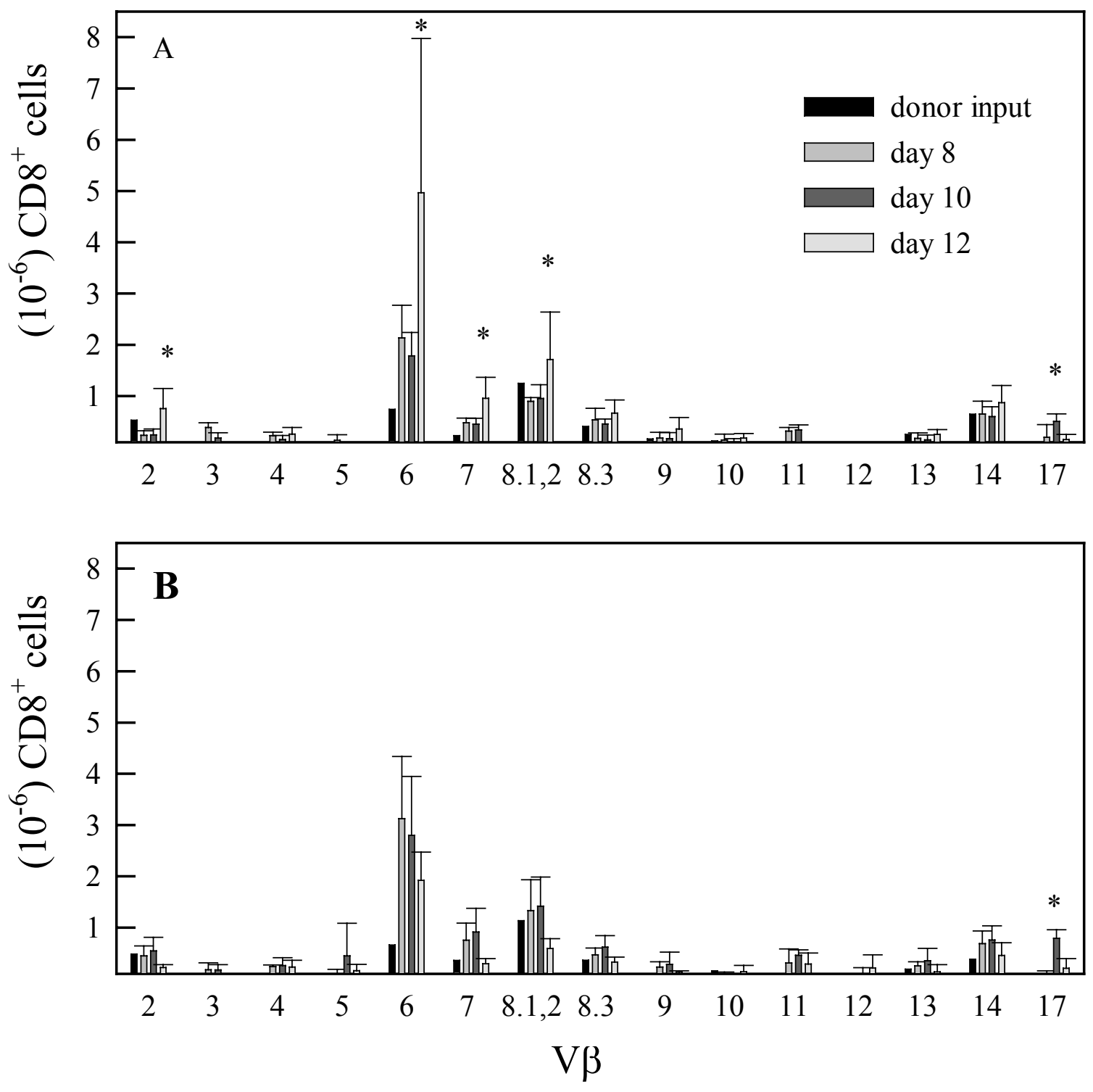
Figure 11. V $\beta$ repertoire of CD8+ $T$ cells recovered from reovirus-infected SCID recipients of immune splenocytes from donor mice infected perorally (A.) or in the hind footpads (B.) one month before. Splenocytes were isolated from recipient reovirus infected SCID mice at days 8, 10, or 12 post-transfer. Data are given as (mean \pm standard deviation) $\times 10^{-6}$.

* Significant by analysis of variance of the $\log _{10}$ ratio of the input cell numbers for each V $\beta$ subpopulation to the numbers recovered. SCID recipients of splenocytes from perorally-infected donors show significantly elevated $\mathrm{V} \beta 2^{+}(p<0.0036), \mathrm{V} \beta 6^{+}(p<0.0144), \mathrm{V} \beta 7^{+}(p<0.0011)$, and Vß8.1, $2^{+}(p<0.0089) \mathrm{CD}^{+} \mathrm{T}$ cells by day 12 post-transfer compared to SCID recipients of cells from footpad-infected donors. Cells recovered from SCID recipients of both perorally-infected and footpad-infected donors showed a significant increase in $\mathrm{V} \beta 17^{+}$cells $(p<0.0272)$ by day 10 post-transfer. 

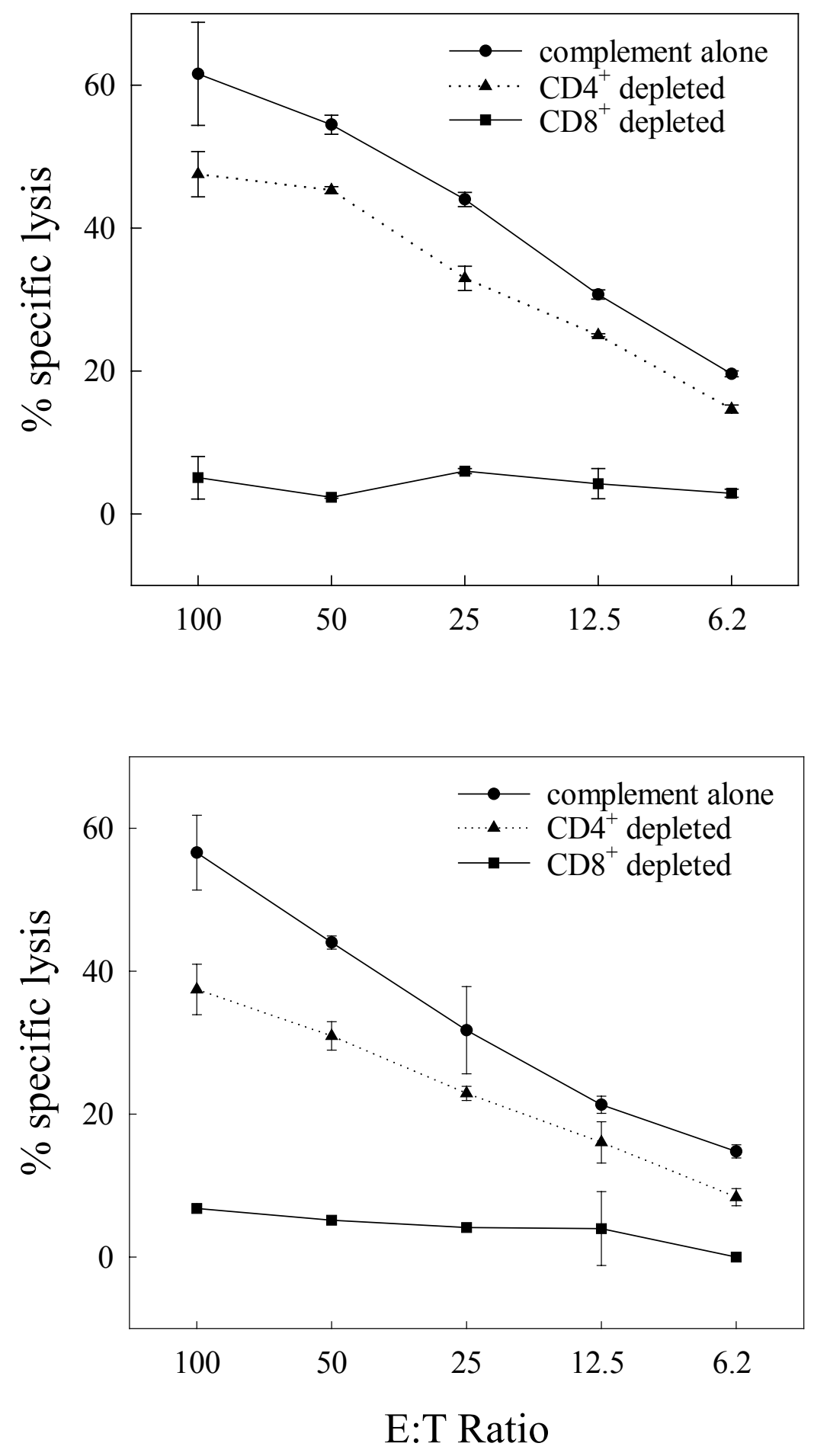
Figure 12. . Immediate ex vivo $51 \mathrm{Cr}$ release assay of splenocytes recovered from reovirus infected C3H SCID recipients ten days after adoptive transfer of purified splenic $\mathrm{T}$ cells from donor $\mathrm{C} 3 \mathrm{H}$ mice previously infected perorally $(\mathrm{A})$ or in the hind footpads $(\mathrm{B})$ with reovirus. Recovered splenocytes were complement depleted of either $\mathrm{CD4}^{+}$cells or $\mathrm{CD8}^{+}$ cells as described in methods. Control fractions were treated with complement alone.

Data are given as mean $\%$ specific lysis \pm standard deviation. 

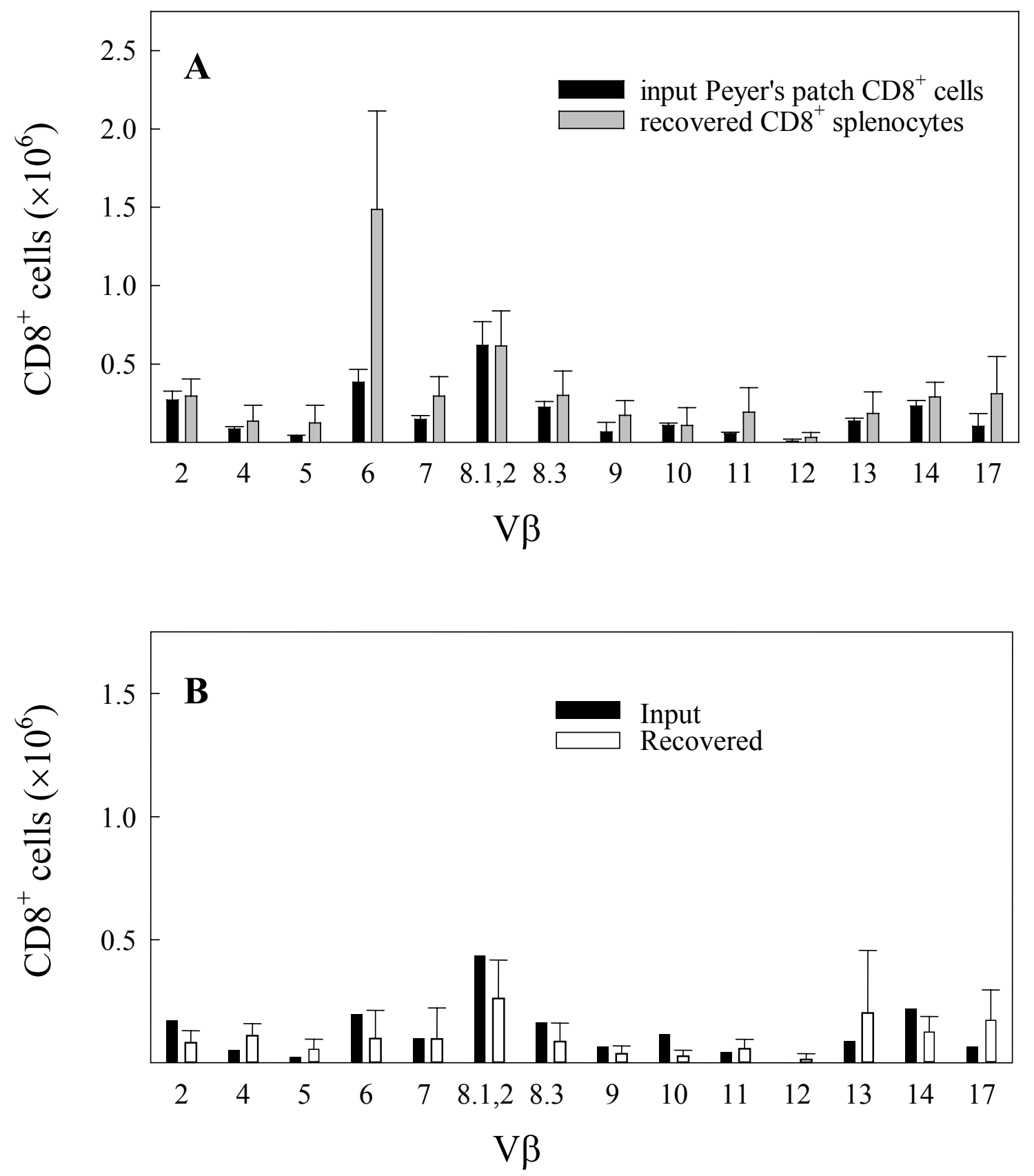
Figure 13. VB repertoire of CD8+ lymphocytes recovered from the spleens of infected $\mathrm{C} 3 \mathrm{H}$ SCID recipient mice of perorally infected (A) or naïve (B) donor $\mathrm{C} 3 \mathrm{H}$ mice, ten days after adoptive transfer of Peyer's patch cells as described in methods. Data are expressed as (means \pm standard deviations) $\times 10^{-6} \mathrm{CD8}^{+}$cells.

Figure $A, n=5$ recipient mice compiled from 2 experiments.

Figure $\mathrm{B}, \mathrm{n}=3$ recipient mice. 


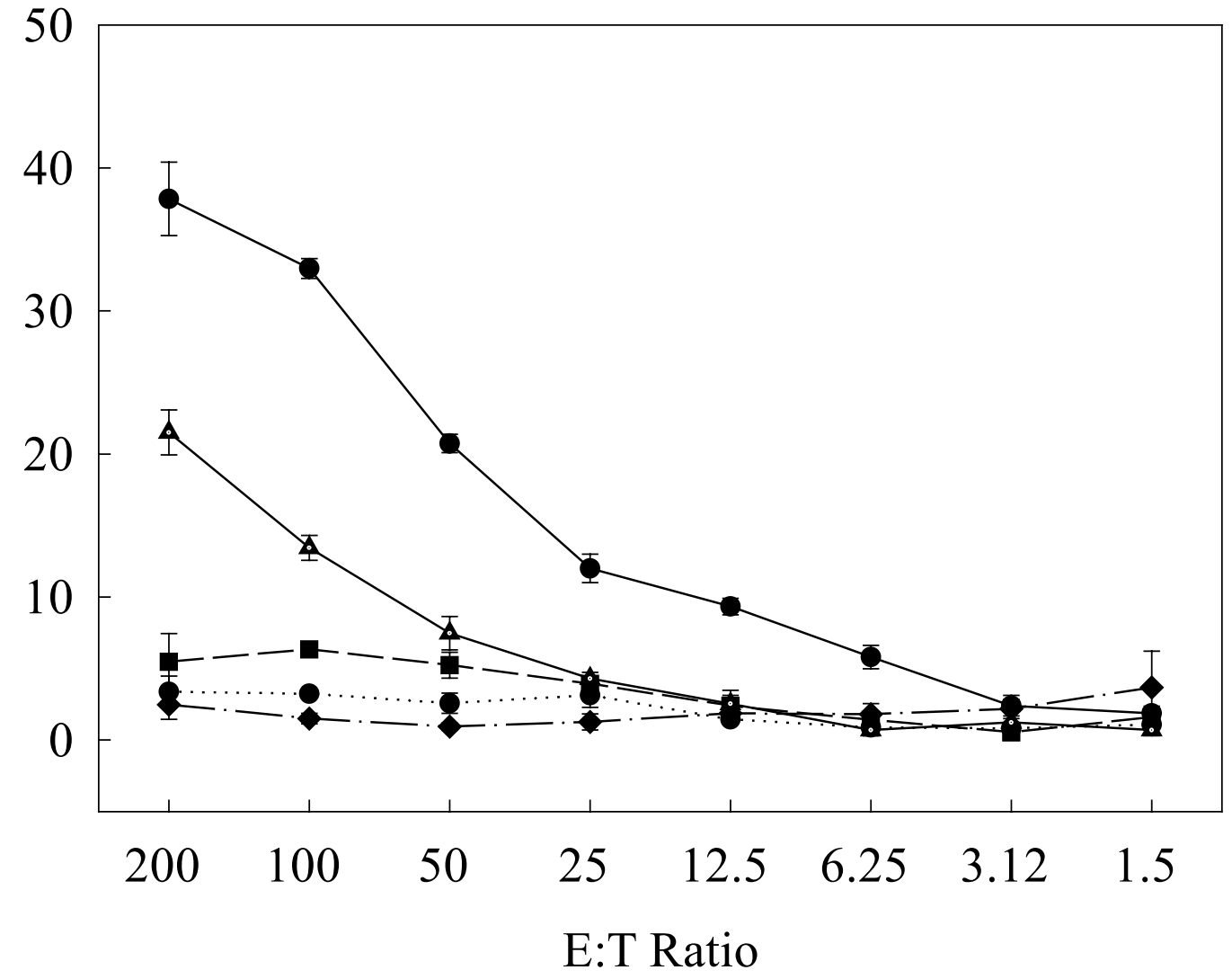


Figure 14. Ex vivo 51Cr release assay of splenocytes from reovirus-infected C3H SCID mice 10 days after adoptive transfer of purified Peyer's patch $\mathrm{T}$ cells from naïve $\mathrm{C} 3 \mathrm{H}$ donors or immune donors infected perorally with reovirus one month previously. Splenocytes from infected SCID recipients of immune donor $\mathrm{T}$ cells mediate virus-specific cytotoxicity immediately ex vivo, whereas splenocytes of infected SCID recipients of naïve $T$ cells do not. 
A popliteal ly mph nodes

B Peyer's patches

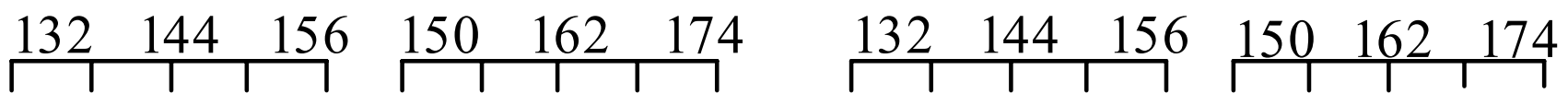
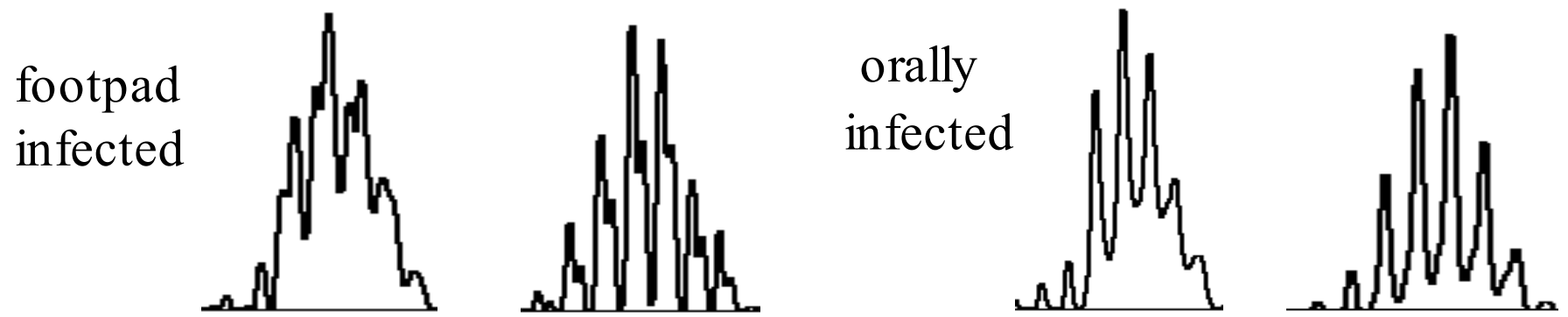

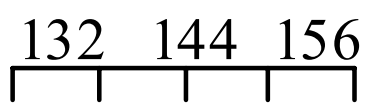
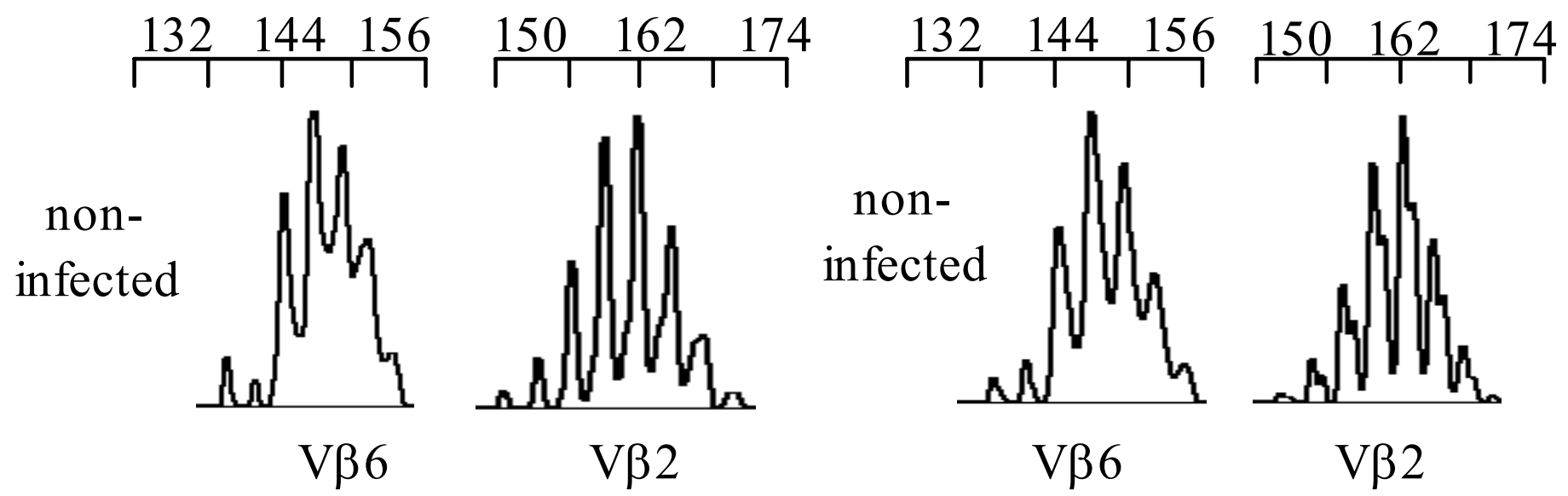
Figure 15. CDR3 length profiles of $\mathrm{V} \beta 6+$ and $\mathrm{V} \beta 2+\mathrm{CD8}+\mathrm{T}$ cells from the popliteal lymph nodes of footpad infected mice (A) and Peyer's patch cells of orally infected mice (B) compared to cells from non-infected controls. Cells from infected and non-infected mice were harvested 10 days after infection, and were depleted of $\mathrm{CD}^{+}$cells. Cellular RNA was isolated and used as template for RT-PCR using V $\beta 2$ or V $\beta 6$ and $C \beta$ primers and assessed using an automated sequencer as described in methods. Data are shown as representative CDR3 $\beta$ length profiles for $\mathrm{V} \beta 6$ and $\mathrm{V} \beta 2 \mathrm{CD8}^{+}$. No differences are seen in the profiles of cells from infected mice compared to those of non-infected mice immediately ex vivo. 

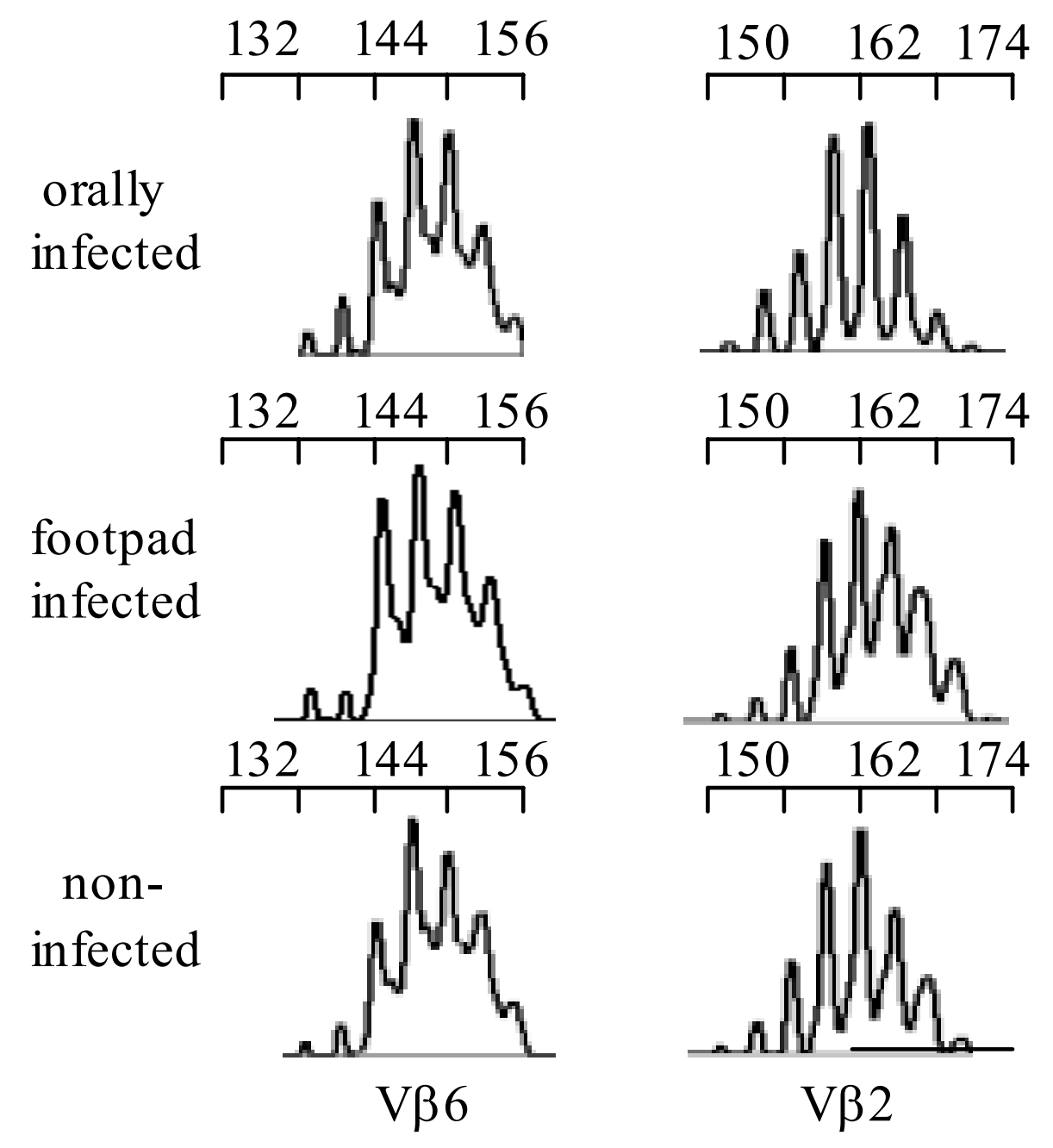
Figure 16. CDR3 $\beta$ length profiles of $\mathrm{V} \beta 6+$ and $\mathrm{V} \beta 2+\mathrm{CD} 8+\mathrm{T}$ cells from the spleens of footpad infected mice and orally infected mice, compared to cells from non-infected controls. Cells from infected and non-infected mice were harvested 10 days after infection, and were depleted of $\mathrm{CD4}^{+}$cells. Cellular RNA was isolated and used as template for RTPCR using V $\beta 2$ or $V \beta 6$ and $C \beta$ primers and assessed using an automated sequencer as described in methods. Data are shown as representative CDR3 $\beta$ length profiles for V $\beta 6$ and $\mathrm{V} \beta 2 \mathrm{CDB}^{+}$. No differences are seen in the profiles of cells from infected mice compared to those of non-infected mice immediately ex vivo. 
Donor input cells
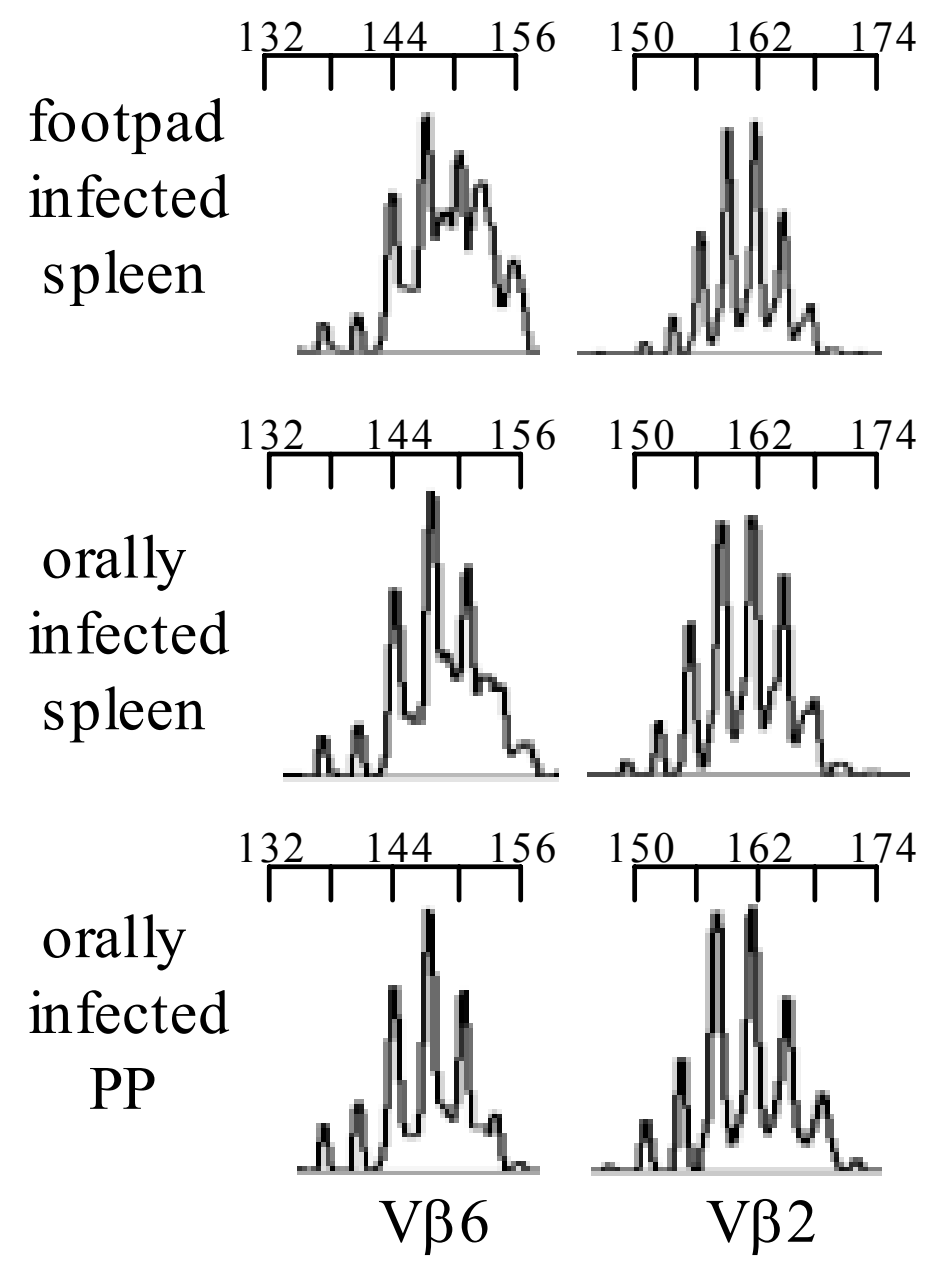

Recovered SCID splenocytes
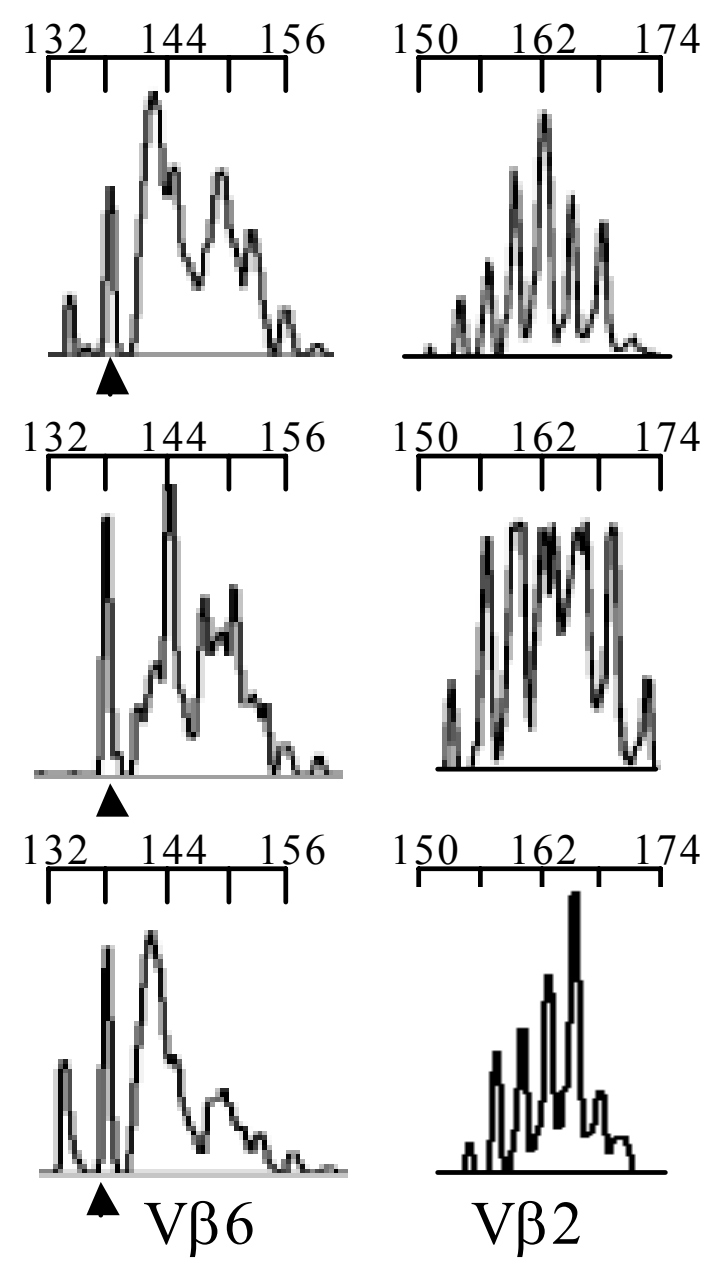
Figure 17. CDR3 $\beta$ length profiles of $\mathrm{V} \beta 6+$ and $\mathrm{V} \beta 2+\mathrm{CD} 8+\mathrm{T}$ cells recovered from the spleens of reovirus infected SCID recipients of purified splenic T cells from footpad infected mice or purified $\mathbf{T}$ cells from the spleens or Peyer's patches of orally infected donor mice. Cells were recovered from recipient mice 10 days post-transfer, and were depleted of $\mathrm{CD}^{+}$cells. Cellular RNA was isolated and used as template for RT-PCR using $V \beta 2$ or $V \beta 6$ and $C \beta$ primers and assessed using an automated sequencer as described in methods. Data are shown as representative CDR3 $\beta$ length profiles for V $\beta 6$ and V $\beta 2 \mathrm{CD8}^{+}$ cells. A consistent expansion of $\mathrm{V}^{+} 6^{+} \mathrm{CD8}^{+}$cells using a TCR $\beta$ chain $\mathrm{CDR} 3 \beta$ length is indicated by the arrow $(\Delta)$. No consistent or clear changes are seen in the control Vß2 profiles. 
2 week cultured splenocytes
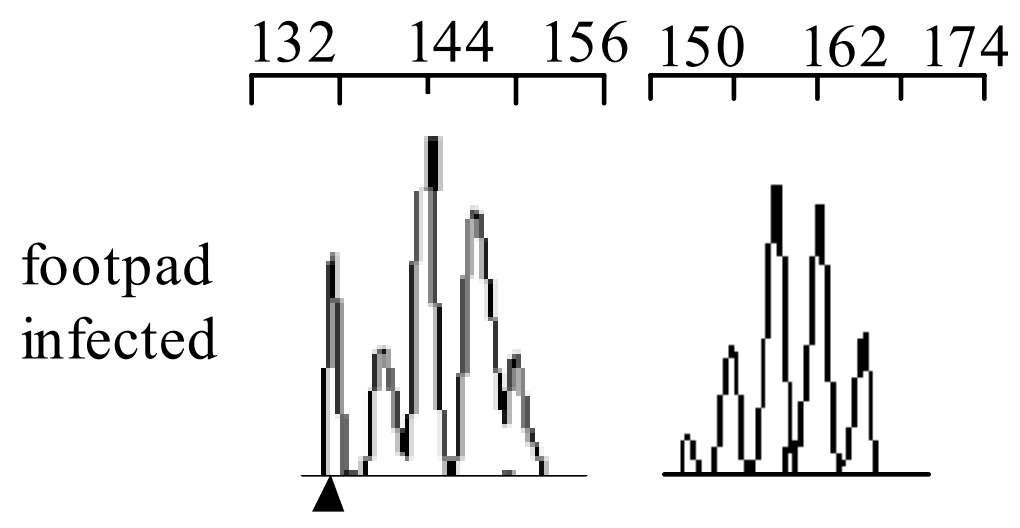

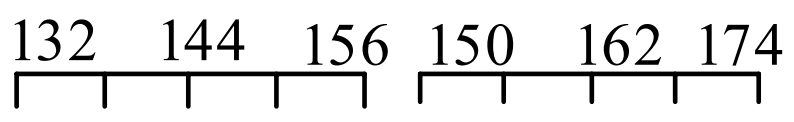

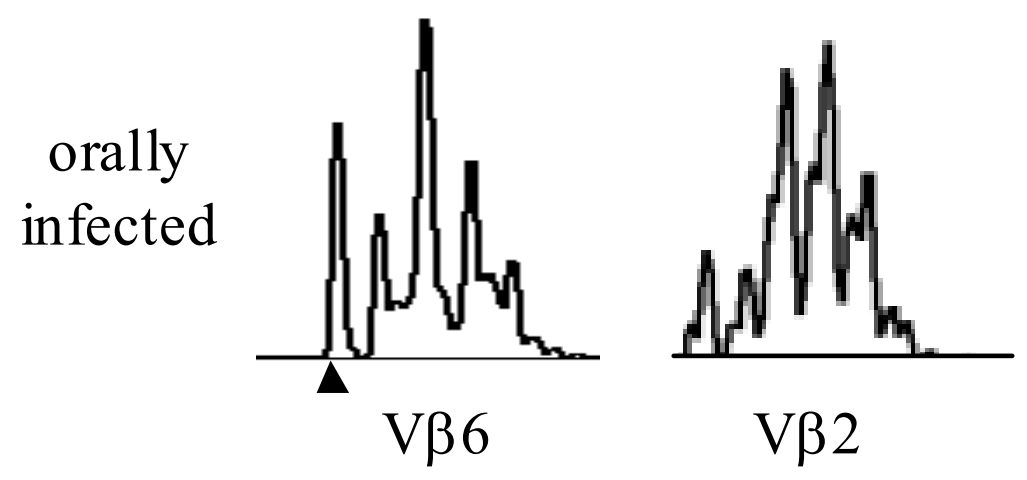


Figure 18. CDR3 $\beta$ length profiles of $\mathrm{V} \beta 6+$ and $\mathrm{V} \beta 2+\mathrm{CD8}+\mathrm{T}$ cells from cultured cell lines established from the spleens of footpad infected mice or orally infected mice. Cells were restimulated in vitro with reovirus for 2 weeks, and were depleted of CD4+ cells prior to analysis. Cellular RNA was isolated and used as template for RT-PCR using V $\beta 2$ or V $\beta 6$ and $C \beta$ primers and assessed using an automated sequencer as described in methods. Data are shown as representative CDR3 $\beta$ length profiles for $\mathrm{V} \beta 6$ and $\mathrm{V} \beta 2 \mathrm{CD8}^{+}$. A consistent expansion of $\mathrm{V}^{+} 6^{+} \mathrm{CD8}^{+}$cells using a TCR $\beta$ chain $\mathrm{CDR} 3 \beta$ length is indicated by the arrow $(\Delta)$. No consistent or clear changes are seen in the control $V \beta 2$ profiles. 
Cell lines from recovered SCID splenocytes

$$
\begin{array}{lll}
132 \quad 144 \quad 156 \\
\Gamma \quad 1 \quad 1
\end{array}
$$
footpad
infected

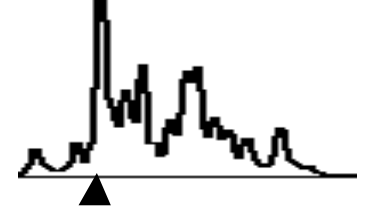

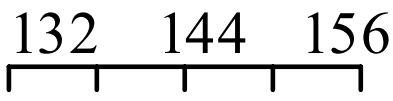

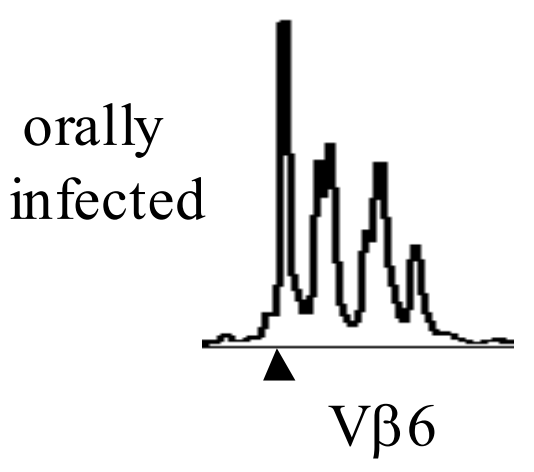

$\mathrm{V} \beta 2$

Not done

$\mathrm{V} \beta 2$

Not done

$\mathrm{V} \beta 2$ 
Figure 19. CDR3 $\beta$ length profiles of $\mathrm{V} \beta 6+\mathrm{CD8}+\mathrm{T}$ cells from cell lines established from recovered splenocytes of reovirus infected SCID recipients of purified splenic $T$ cells from footpad infected mice or purified $T$ cells from the spleens of orally infected donor mice. Cells were recovered from recipient mice 10 days post-transfer, and were depleted of $\mathrm{CD4}^{+}$ cells. The cells were re-stimulated with reovirus in vitro for a period of 3 weeks. Cellular RNA was then isolated and used as template for RT-PCR using V $\beta 6$ and $C \beta$ primers and assessed using an automated sequencer as described in methods. Data are shown as CDR3 $\beta$ length profiles for $\mathrm{V} \beta 6 \mathrm{CD8}^{+}$cells. A dramatic and overwhelming expansion of $\mathrm{V}^{+} 6^{+} \mathrm{CD8}^{+}$ cells using a TCR $\beta$ chain CDR3 $\beta$ length is indicated by the arrow $(\Delta) . V \beta 2$ profiles were not assessed, as the cell lines from recipients of footpad infected donors and orally infected donors were shown to be $100 \%$ and $65 \% \mathrm{~V} \beta 6^{+}$, respectively. 


\section{References}

Arenz, M., Herzog-Hauff, S., Meyer Zum Buschenfelde, K.H., and H.F. Lohr. 1997. Antigenindependent in vitro expansion of T cells does not affect the T cell receptor $\mathrm{V}$ beta repertoire. $J$. Mol. Med. 75:678-686.

Battegay, M., Moskophidis, D., Rahemtulla, A., Hengartner, H., Mak, T.W., and R.M. Zinkernagel. 1994. Enhanced establishment of a viral carrier state in adult CD4+ T-cell-deficient mice. J. Virol. 68:4700-4704.

Bode, U., Duda, C., Weidner, F., Rodriguez-Palmero, M., Wonigeit, K., Pabst, R., and J. Westermann. 1999. Activated T cells enter rat lymph nodes and Peyer's patches via high endothelial venules: survival by tissue-specific proliferation and preferential exit of CD8+ T cell progeny. Eur. J. Immunol. 29:1487-1495.

Bodkin, D.K., Nibert, M.L., and B.N. Fields. 1989. Proteolytic digestion of reovirus in the intestinal lumens of neonatal mice. J. Virol. 63:4676-4681.

Bousso, P., Levraud, J.P., Kourilsky, P., and J.P. Abastado. 1999. The composition of a primary T cell response is largely determined by the timing of recruitment of individual T cell clones. $J$. Exp. Med. 189: 1591-1600. 
Campbell, N., Yio, X.Y., So, L.P., Li, Y., and L. Mayer. 1999. The intestinal epithelial cell: processing and presentation of antigen to the immune system. Immunol. Rev. 172:315-324. Chen, D., Lee, F., Cebra, J.J., and D.H. Rubin. 1997. Predominant T cell receptor Vbeta usage of intraepithelial lymphocytes during the immune response to enteric reovirus infection. $J$. Virol. $71: 3431-3436$.

Cole, G.A., Hogg, T.L., and D.L. Woodland. 1995. T cell recognition of the immunodominant Sendai virus NP324-332/Kb epitope is focused on the center of the peptide. J. Immunol. $155: 2841-2848$.

Cuff, C.F., Cebra, C.K., Rubin, D.H., and J.J. Cebra. 1993. Developmental relationship between cytotoxic alpha/beta T cell receptor-positive intraepithelial lymphocytes and Peyer's patch lymphocytes. Eur. J. Immunol. 23:1333-1339.

Cuff, C.F., Fulton, J.R., Barnett, J.B., and C.S. Boyce. 1998. Enteric reovirus infection as a probe to study immunotoxicity of the gastrointestinal tract. Toxicol. Sci. 42:99-108.

Cuff, C.F., Lavi, E., Cebra, C.K., Cebra, J.J., and D.H. Rubin. 1990. Passive immunity to fatal reovirus serotype 3-induced meningoencephalitis mediated by both secretory and transplacental factors in neonatal mice. J. Virol. 64:1256-1263. 
Farstad, I.N., Halstensen, T.S., Lien, B., Kilshaw, P.J., and A.I. Lazarovitz. 1996. Distribution of $\beta 7$ integrins in the human intestinal mucosal and organized gut-associated lymphoid tissue. Immunology. 89:227-237.

Finberg, R., Weiner, H.L., Fields, B.N., Benacerraf, B., and S.J. Burakoff. 1979. Generation of cytolytic T lymphocytes after reovirus infection: role of the S1 gene. Proc. Natl. Acad. Sci. USA. $76: 442-446$.

George, A., Kost, S.I., Witzleben, C.L., Cebra, J.J., and D.H. Rubin. 1990. Reovirus-induced liver disease in severe combined immunodeficient (scid) mice. A model for the study of viral infection, pathogenesis, and clearance. J. Exp. Med. 171:929-934.

Haanen, J.B., Toebes, M., Cordaro, T.A., Wolkers, M.C., Kruisbeek, A.M., and T.N. Schumacher. 1999. Systemic $\mathrm{T}$ cell expansion during a localized viral infection. Eur. J. Immunol. 29:1168-1174.

Hershberg, R.M., and L.F. Mayer. 2000. Antigen processing and presentation by cells- intestinal epithelial polarity and complexity. Immunol. Today. 21: 123-128.

Huang, F.D., Platt, N., Wykes, M., Major, J.R., Powell, T.J., Jenkins, C.D., and G.G. MacPherson. 2000. A discrete subpopulation of dendritic cells transports apoptotic intestinal epithelial cells to the T cell areas of the mesenteric lymph nodes. J. Exp. Med. 191:435-444. 
Ishikawa, H., Saito, H., Suzuki, K., Oida, T., and Y. Kanamori. 1999. New gut-associated lymphoid tissue cryptopatches breed murine intestinal intraepithelial T cell precursors. Immunol. Res. 20:243-250.

Kaiserlian, D., and N. Etcharct. 1999. Entry sites for oral vaccines and drugs: A role for M cells, enterocytes and dendritic cells? Semin Immunol. 11:217-224.

Kang, H.K., Mikszta, J.A., Deng, H., Sercarz, E.E., Jensen, P.E., and B.S. Kim. 2000. Processing and reactivity of $\mathrm{T}$ cell epitopes containing two cysteine residues from hen egg white lysozyme (HEL74-90). J. Immunol. 164:1775-1782.

Kelsall, B.L., and W. Strober. 1996. Distinct populations of dendritic cells are present in the subepithelial dome and T cell regions of the murine Peyer's patch. J. Exp. Med. 183:237-247.

Lefrancois, L., Parker, C.M., Olson, S., Muller, W., Wagner, N., Schon, M.P., and L. Puddington. 1999. The role of beta 7 integrins in CD8 $\mathrm{T}$ cell trafficking during an antiviral immune response. J. Exp. Med. 189:1631-1638.

Letvin, N.L., Kauffman, R.S., and R. Finberg. 1981. T lymphocyte immunity to reovirus: cellular requirements for generation and role in clearance of primary infections. J. Immunol. 127:23342339. 
London, S.D., Rubin, D.H., and J.J. Cebra. 1987. Gut mucosal immunization with reovirus serotype 1/L stimulates virus-specific cytotoxic $\mathrm{T}$ cell precursors as well as IgA memory cells in Peyer's patches. J. Exp. Med. 165:830-847.

London, S.D., Cebra, J.J., and D.H. Rubin. 1989. Intraepithelial lymphocytes contain virusspecific, MHC-restricted cytotoxic cell precursors after gut mucosal immunization with reovirus serotype 1/Lang. Reg. Immunol. 2:98-102.

Major, A.S., and C.F. Cuff. 1996. Effects of the route of infection on immunoglobulin G subclasses and specificity of the reovirus-specific humoral immune response. J. Virol. 70:59685974.

Matloubian, M., Concepcion, R.J., and R. Ahmed. 1994. CD4+ T cells are required to sustain CD8+ cytotoxic T-cell responses during chronic viral infection. J. Virol. 68:8056-8063.

Matsumoto, S., Setoyama, H., and Y Umesaki. 1992. Differential induction of major histocompatibility complex molecules on mouse intestine by bacterial colonization. Gastroenterology. 103:1777-1782.

Moghaddami, M., Cummins, A., and G. Mayerhofr. 1998. Lymphocyte-filled villi: comparisons with other lymphoid aggregations in the mucosal of the human small intestine. Gastroenterology. 115:1414-1425. 
Muller, S., Buhler-Jungo, M., and C. Mueller. 2000. Intestinal intraepithelial lymphocytes exert potent protective cytotoxic activity during an acute viral infection. J. Immunol. 164:1986-1994.

Palmer, E.L., and M.L. Martin. 1977. The fine structure of the capsid of reovirus type 3. Virology. 76:109-113.

Pang, K.Y., Walker, W.A., and K.J. Bloch. 1981. Intestinal uptake of macromolecules. Differences in distribution and degradation of protein antigen in control and immunized rats. Gut. 22:1018-1024.

Pannetier, C., Cochet, M., Darche, S., Casrouge, A., Zoller, M., and P. Kourilsky. 1993. The sizes of the CDR3 hypervariable regions of the murine $\mathrm{T}$ cell receptor beta chains vary as a function of the recombinant germ-line segments. Proc. Natl. Acad. Sci. USA. 90:4319-4323.

Periwal, S.B., and J.J. Cebra. 1999. Respiratory mucosal immunization with reovirus serotype 1/L stimulates virus-specific humoral and cellular immune responses, including double-positive (CD4 (+)/CD8 (+)) T cells. J. Virol. 73:7633-7640.

Rocha, B., Dautigny, N., and P. Pereira. 1989. Peripheral T lymphocytes: expansion potential and homeostatic regulation of pool sizes and CD4/CD8 ratios in vivo. Eur. J. Immunol. 19:905911.

Rubin, D.H., Kornstein, M.J., and A.O. Anderson. 1985. Reovirus serotype 1 intestinal infection: a novel replicative cycle with ileal disease. J. Virol. 53:391-398. 
Rudolphi, A., Spiess, S., Conradt, P., Claesson, M.H., and J. Reimann. 1991. CD3+ T cells in severe combined immune deficiency (scid) mice. I. Transferred purified CD4+ T cells, but not CD8+ T cells are engrafted in spleen of congenic scid mice. Eur. J. Immunol. 21:523-533.

Saito, H., Kanamori, Y., Takemori, T., Nariuchi, H., Kubota, E., Takahashi-Iwanaga, H., Iwanaga, T., and H. Ishikawa. 1998. Generation of intestinal T cells from progenitors residing in gut cryptopatches. Science. 280:275-278.

Slifka, M.K., Matloubian, M., and R. Ahmed. 1995. Bone marrow is a major site of long-term antibody production after acute viral infection. J. Virol. 69:1895-1902.

Smith, R.E., Zweerink, H.J., Joklik, W.K. 1969. Polypeptide components of virions, top component and cores and reovirus type 3. Virology. 39:791-810.

So, A.L., Small, G., Sperber, K., Berker, K., Oei, E., Tyorkin, M., and L. Mayer. 2000. Factors affecting antigen uptake by human intestinal epithelial cell lines. Dig. Dis. Sci. 45:1130-1137.

Taterka, J., Cebra, J.J., and D.H. Rubin. 1995. Characterization of cytotoxic cells from reovirus infected SCID mice: activated cells express natural killer- and lymphokine-activated killer-like activity but fail to clear infection. J. Virol. 69:3910-3914. 
Thompson, A.H., London, L., Bellum, S.C., Hamamdzic, D., Harley, R.A., and S.D. London. 1996. Respiratory-mucosal lymphocyte populations induced by reovirus serotype 1 infection. Cell. Immunol. 169:278-287.

Weinstein, P.D., and J.J. Cebra. 1991. The preference for switching to IgA expression by Peyer's patch germinal center B cells is likely due to the intrinsic influence of their microenvironment. $J$. Immunol. 147:4126-4135.

Wolf, J.L., Rubin, D.H., Finberg, R., Kauffman, R.S., Sharpe, A.H., Trier, J.S., and B.N. Fields. 1983. Intestinal M cells: a pathway for entry of reovirus into the host. Science. 212:471-472.

Zimmermann, C., and H. Pircher. 1999. A novel approach to visualize polyclonal virus-specific CD8 T cells in vivo. J. Immunol. 162:5178-5182.

Zimmermann, C., Prevost-Blondel, A., Blaser, C., and H. Pircher. 1999. Kinetics of the response of naïve and memory CD8 T cells to antigen: similarities and differences. Eur. J. Immunol. 29:284-290. 


\title{
Preservation of Intestinal Immunity to Reovirus Infection in Ageing
}

\author{
Jonathan R. Fulton and Christopher F. Cuff \\ Department of Microbiology and Immunology, West Virginia University \\ School of Medicine
}




\begin{abstract}
Systemic immunity is progressively impaired in ageing, predisposing to morbidity and mortality from neoplasia and infectious disease. However, the effect of ageing on mucosal immunity is controversial. To assess intestinal immunity in ageing, young and aged mice were orally immunized with reovirus serotype 1 strain Lang or cholera toxin (CT). Analysis of local intestinal and systemic reovirus or CT-specific IgA and IgG responses was performed by ELISPOT analysis of Peyer's patches (PP) and spleens, and by ELISA of intestinal fragment culture supernatant and fecal IgA and serum IgG. Reovirus-specific cytotoxic T lymphocyte (CTL) responses were assessed by frequency analysis and bulk culture ${ }^{51} \mathrm{Cr}$ release assay. As previously reported by others, aged mice immunized orally with CT mounted diminished intestinal and systemic IgA responses to CT compared to young mice. By contrast, aged mice yielded two to three-fold more reovirus-specific IgA-producing cells in the PP compared to young mice, and also higher titers of reovirus-specific IgA in fragment culture supernatants. In addition, reovirus-specific cytotoxicity and CTL frequencies from aged mice were not different from those of young mice. Together, these results suggest a diminished potential for systemic and intestinal immunity to orally applied protein antigens in ageing, but an intact ability to respond to intestinal virus infection. Infection with a replicating virus may induce inflammatory mediators and innate immune factors that potentiate the priming of mucosal immunity, overcoming ageing related deficits otherwise seen following oral immunization with nonreplicating or inert antigens, thus suggesting the importance of antigen replication to antigenspecific immunotherapy strategies in the elderly.
\end{abstract}

Key words: ageing, GALT, mucosal, CTL, IgA, IgG, Peyer's patch, intestine, reovirus, cholera toxin 


\section{Introduction}

Ageing is associated with a decline of immune function in humans and animals. Declining immune function and regulation is believed to result in an increased incidence of infectious diseases, neoplasia, and autoimmunity in the aged (1). Ageing affects cellular immunity mediated by both $\mathrm{CD} 4^{+}$and $\mathrm{CD} 8^{+} \mathrm{T}$ cells, and $\mathrm{CD} 8^{+} \mathrm{CTL}(2)$. A number of studies have documented decreased CTL proliferation and killing in aged mice $(3,4)$ and humans $(5-7)$, that can lead to prolonged duration of infection (8). Impaired CTL proliferation results from decreased T helper (Th) cell support (9), diminished antigen presenting cell function (10), and defective cell signaling following TCR/CD3 ligation (11-13) and CD28 co-stimulation (14). Decreased cytotoxicity by CTL results from defective production of perforin (15-17) or serine esterase gene products (15), and possibly impaired exocytosis of cytotoxic granule contents (18). In some studies, naive precursor CTL (pCTL) frequencies are lower in aged animals and humans following infection or immunization compared to the pCTL frequencies in similarly treated young animals (19-22). By contrast, other studies suggest that the initial frequencies of antigenspecific pCTL may be similar in young and aged animals, but pCTL from aged subjects may undergo far fewer rounds of proliferation than those from young, generating a smaller overall response magnitude in the aged $(10,23)$. CTL that are generated in aged subjects may show similar (24) or decreased (4) levels of cytotoxicity on a per-cell basis, but typically produce diminished amounts of IFN- $\gamma$ compared to CTL from young subjects, possibly as a result of a shifting Th type 1 to a type 2 immune predilection in ageing $(7,25,26)$.

Humoral responses to both B cell mitogens and specific antigens such as CT (27), keyhole limpet hemocyanin, and sheep red blood cells (28) are also diminished in aged animals and humans. It is believed that these failings are due more to acquired defects in Th cell function 
than to intrinsic defects in the responder B cells themselves $(29,30)$. Evidence does exist, however, that some murine B cell populations do acquire defects with ageing in cell cycle progression following stimulation by autoreactive T cells (31) or B cell mitogens (32).

Although many of the studies that have shown immune hyporesponsiveness have focused on systemic immunity, there is evidence that mucosa-associated lymphoid tissues may retain immune function longer than systemic immune tissues. For example, Gallichan and Rosenthal (33) showed that mice infected intranasally with a recombinant adenovirus vector containing the herpes simplex virus glycoproteins B ( $\mathrm{gB}$ ) mounted both mucosal and systemic CTL responses, and these mice maintained detectable gB-specific CTL in mucosal lymphoid tissue, although not in systemic lymphoid tissue, for longer than 1.5 years following immunization. Mucosal humoral immunity may also be differentially affected by ageing compared to systemic immunity. Kawanishi et al. (34) found preservation, and/or enhancement of intestinal IgA responses to orally administered Mycobacterium tuberculosis antigen generated in the Peyer's patches (PP) and the mesenteric lymph nodes of aged mice compared to young. These findings were interpreted as a lack of $\mathrm{CD}^{+}{ }^{+}$suppressor $\mathrm{T}$ cell function, as co-culture experiments of aged PP B cells and Th cells primed in vivo with $\mathrm{PP} \mathrm{CD} 8^{+}$cells from young mice did not overproduce antigen-specific IgA, whereas similar co-cultures using young PP B cells and Th cells with aged $\mathrm{CD}^{+}$cells did overproduce IgA. This hyper-responsiveness was reversible by treatment with exogenous IL-2 (35), a protocol that had been previously shown to compensate for decreased Th cell function in ageing (36). However, a recent report by Koga et al. (37) indicated that murine mucosal IgA responses to orally administered CT and ovalbumin progressively declined with increasing age. The results suggested that defective induction of humoral and Th cell responses was more pronounced, and began at an earlier age in mice given CTB or ovalbumin by an oral 
route than those immunized systemically. Thus, the effects of ageing on immune responses to mucosal immunization remain controversial.

In this report we examine CTL and humoral responses in aged mice following oral infection with the model enteric pathogen, reovirus, which induces CTL in PP and spleen as well as humoral immunity following oral infection of immunocompetent mice (38). Our data suggest the ageing intestinal immune system remains as capable of responding to virus infection as that of the young, and that there is no diminution of anti-viral CTL or B cell priming or function in the intestinal immune tissues with ageing. The preservation of immune responsiveness to oral reovirus infection suggests that this route may prove efficacious in vaccination and immunotherapeutic strategies in the aged.

\section{Methods}

Mice: Male mice were used in all studies. Balb/c mice, age 18-24 months, were born in our animal facility and housed in individual micro-isolator cages in a pathogen-free room. Additional Balb/c mice, age 22 months, and B6C3F1 mice, age 18-22 months, were obtained from the National Institutes of Ageing (Bethesda, MD). C3HeB/FeJ mice were obtained from Jackson Laboratories (Bar Harbor, MA) at 8 weeks of age. Young Balb/c and B6C3F1 mice were obtained at 8 weeks of age from Charles River (Wilmington, MA), and Harlan Laboratories (Indianapolis, IN), respectively, and allowed to acclimate for 1 week before use. All animals were kept in micro-isolator cages in a pathogen-free room and maintained on autoclaved food and water. Virus-infected animals were housed in a laminar flow hood in a separate room under negative pressure. 
Virus: Third passage reovirus serotype 1 strain Lang (T1L) was grown in L929 cells and purified by freon extraction followed by $\mathrm{CsCl}$ gradient centrifugation as described (39). Infectious virus titers were assessed by plaque assay in L929 cells (40).

Animal infections: Mice were infected orally with $3 \times 10^{7}$ plaque-forming units (pfu) of reovirus in a vehicle of $50 \mu 1$ of sterile borate-buffered saline and gelatin by gavage. Systemically infected mice subcutaneously received $3 \times 10^{7}$ pfu of reovirus in $100 \mu 1$ of vehicle.

Cholera toxin immunizations: Mice were orally immunized with $10 \mu \mathrm{g}$ of azide-free CT (List Biological Laboratories, Campbell, CA) in 50 $\mu$ l sterile PBS by gavage on days 0, 7 and 14 .

Preparation and culture of splenic and PP cells: Single cell suspensions of spleens were prepared by expression through sterile nylon mesh. Splenocytes were depleted of red blood cells by incubation in a hypotonic solution of $\mathrm{NH}_{4} \mathrm{Cl}$ and Tris- $\mathrm{HCl}$. Splenocytes were washed 3 times in tissue culture medium (TCM) consisting of RPMI-1640 (Biowhittaker, Walkersville, MD) supplemented with 10\% FBS (Hyclone, Logan,VT) 2mM L-glutamine, 100U penicillin/ml, $0.1 \mu \mathrm{g}$ streptomycin $/ \mathrm{ml}$, and $50 \mu \mathrm{M} \beta$-mercaptoethanol. Single-cell suspensions of PP were prepared by mechanical dissociation using 18-gauge needles. CTL were generated by restimulating splenocytes and PP cells with reovirus T1L at a multiplicity of infection (MOI) of 1 for $1 \mathrm{hr}$ at $25^{\circ} \mathrm{C}$. Cells were resuspended in TCM to a final concentration of $2 \times 10^{6}$ cells $/ \mathrm{ml}$ and incubated at $37^{\circ} \mathrm{C}$ in an atmosphere of $5 \% \mathrm{CO}_{2}$.

Preparation of bone marrow cells: Femurs and tibiae were excised, and the ends were removed with scissors. Cold Hank's buffered salt solution (HBSS) (Sigma, St. Louise, MO) was forced through the length of the bones by means of a 26-gauge needle attached to a $3 \mathrm{ml}$ syringe inserted into one cut end of the bone. The contents were flushed into $15 \mathrm{ml}$ centrifuge tubes. The 
marrow plug was rendered a single-cell suspension by expression through nylon mesh and resuspended in TCM. Viability was determined by trypan blue exclusion.

Bulk 51Cr release assay: Assays were performed as previously described (41). Briefly, Ficoll-Hypaque gradient-purified effector CTLs were added to triplicate wells of reovirusinfected or non-infected ${ }^{51}$ Cr-labeled L929 $\left(\mathrm{H}-2^{\mathrm{k}}\right)$ or KD2SV $\left(\mathrm{H}-2^{\mathrm{d}}\right)$ target cells in a V-bottom 96-well plate (Nalgene) at effector: target cell ratios starting at 25:1. The assay plates were incubated for $4 \mathrm{hrs}$ at $37^{\circ} \mathrm{C}$ in an atmosphere of $5 \% \mathrm{CO}_{2}$. The cells were then pelleted by centrifugation, and $100 \mu \mathrm{l}$ of supernatant was removed from each well. Specific lysis of reovirus infected targets for each effector: target ratio was calculated as described (38).

Limiting dilution analysis: Splenocytes were diluted to $2.4 \times 10^{6} / \mathrm{ml}$ in TCM. Replicates $(n=24)$ were serially diluted two-fold in U-bottomed 96-well culture plates (Costar, Corning NY). To each well $2.5 \times 10^{4}$ syngeneic thioglycollate-elicited peritoneal macrophages previously pulsed with reovirus T1L and irradiated with 2000 rad of gamma radiation were added in $60 \mu 1$ TCM. The plates were incubated overnight at $37^{\circ} \mathrm{C}$ in an atmosphere of $5 \% \mathrm{CO}_{2}$, whereupon 20 $\mu 1$ of IL-2 containing supernatant from concanavalin A-stimulated rat splenocytes, and an equal volume of $1 \mathrm{mM} \alpha$-methylmannoside in RPMI-1640, were added to each well to give a final volume of $200 \mu \mathrm{l}$. The plates were incubated at $37^{\circ} \mathrm{C}$ in an atmosphere of $5 \% \mathrm{CO}_{2}$ for 4 more days, whereupon the contents of each well was split into duplicate wells of 96-well V-bottom plates (Nalgene, Rochester, NY). To each well, $5 \times 10^{3}{ }^{51} \mathrm{Cr}$ (Dupont, Wilmington, DE)-labeled syngeneic target cells, either infected or not infected with reovirus, were added in a volume of $100 \mu \mathrm{l}$ of TCM. The plates were incubated for $4 \mathrm{hrs}$ at $37^{\circ} \mathrm{C}$ in an atmosphere of $5 \% \mathrm{CO}_{2} .100 \mu 1$ of supernatant was removed from each well and the specific activity of each supernatant sample was determined using a Clinigamma gamma counter (Wallac, Gaithersburg, MD). Positive wells 
were determined as ${ }^{51} \mathrm{Cr}$ activity in the supernatant from the plate incubated with infected targets being at least 3 standard deviations above the spontaneous release of reovirus-infected target cells, and simultaneously not showing such activity in the corresponding well of the plate incubated with non-infected targets. The frequencies of virus-specific pCTLs were then determined by the $\chi^{2}$ minimization method of Taswell (42).

Elispot: Elispot analysis of reovirus-specific IgA and IgG-producing cells and cholera toxin B subunit (CTB) specific IgA-producing cells was performed by incubating various numbers of splenic, PP and bone marrow mononuclear cells for 4 hrs on ELISPOT plates (Millipore, Bedford, MA) previously coated with reovirus as described (43), or recombinant CTB subunit (List Biological Laboratories). The plates were then washed 8 times with $0.5 \%$ Tween 20 in PBS. Alkaline-phosphatase conjugated goat anti-mouse IgG or IgA antibodies (Southern Biotechnology, Birmingham, AL) diluted 1:200 in PBS + 5\% FBS were then added to individual wells, and the plates were incubated at $4^{\circ} \mathrm{C}$ overnight. Spots were developed with $100 \mu 1$ of BCIP-NBT substrate (Sigma) in de-ionized $\mathrm{H}_{2} \mathrm{O}$.

\section{Flow cytometric analysis of splenocytes and bone marrow mononuclear cells:}

Constant numbers of mononuclear splenocytes and bone marrow cells were stained with a phycoerythrin (PE)-conjugated anti-CD8 $\alpha \mathrm{mAb}$ (Caltag, Burlingame, CA), fluorescein isothiocyanate (FITC)-conjugated anti- CD4, FITC-conjugated anti-surface immunoglobulin, or PE-conjugated anti-B220 (Pharmingen, San Diego, CA). Cells were analyzed with a FACScan flow cytometer (Becton-Dickinson, Franklin Lakes, NJ), and data were assessed using WinMIDI 2.8 software (Joseph Trotter, Scripps Research Institute, La Jolla, CA).

Preparation of PP and lamina propria (LP) fragment cultures, and fecal extracts: Fragmented PP and LP were cultured by a modified version of the method of Logan et al. (44). 
PP were removed from the small intestine and washed in RPMI- $1640+5 \%$ bovine calf serum. Patches from individual mice were cultured in $2 \mathrm{ml}$ of TCM for 5 days at $37^{\circ} \mathrm{C}$ in an atmosphere of $5 \% \mathrm{CO}_{2}$. Fecal extracts were generated from pellets formed 7 days after the final immunization as described (45).

LP cultures were generated following the removal of the PP by longitudinal cutting of the small intestine followed by transverse cuts to yield $3-5 \mathrm{~cm}$ long segments. Intestinal epithelial cells were removed by 2 successive 30 minute incubations at $37^{\circ} \mathrm{C}$ in $1 \mathrm{mM} \mathrm{EDTA} \mathrm{in} \mathrm{Ca}^{2+}$ and $\mathrm{Mg}^{2+}$-free HBSS with gentle stirring. Fragments from individual mice were then washed and cultured together in $5 \mathrm{ml} \mathrm{TCM}$ for 5 days as above. Supernatants from day 5 PP and LP cultures were collected, clarified by centrifugation, and frozen at $-20^{\circ} \mathrm{C}$ prior to analysis.

ELISA: Assessment of reovirus-specific IgA from LP fragment culture supernatants and virus-specific serum IgG was done by ELISA as described by Major and Cuff (43). Briefly, high protein binding ELISA plates (Costar) were coated with $50 \mu$ l per well of $10^{10}$ particles $/ \mathrm{ml}$ of reovirus T1L or $1 \mu \mathrm{g} / \mathrm{ml}$ goat anti-mouse immunoglobulin heavy and light chain, (Southern Biotechnology) in $0.1 \mathrm{M} \mathrm{NaHCO}_{3}$. CT- specific IgA from fragment culture supernatants and fecal extracts, and CT-specific serum IgG were assessed on ELISA plates coated with $5 \mu \mathrm{g} / \mathrm{ml}$ CTB in PBS. $100 \mu 1$ of serial four-fold dilutions of supernatant samples, fecal extracts, or a purified murine IgA standard (Southern Biotechnology) in PBS $+10 \%$ FCS were added to triplicate wells, and subsequently incubated with $100 \mu \mathrm{l} /$ well of $1 \mu \mathrm{g} / \mathrm{ml}$ biotinylated goat anti-mouse $\mathrm{IgA}$ (Southern Biotechnology). Plates were incubated in $0.25 \mu \mathrm{g} / \mathrm{well}$ avidin-conjugated peroxidase

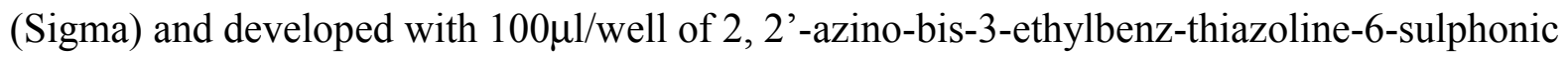
acid (Sigma) as per manufacturer's instructions. Colorimetric analysis was performed at $405 \mathrm{~nm}$ on an ELISA microplate reader (Bio-Tek Instruments Inc., Winooski, VT.) 
Statistical analysis: Means and standard deviations of pCTL frequency, IgA and IgGproducing cells, and mononuclear cell numbers recovered from PP and spleens were compared by Student's t-test using Sigmastat 2.0 software (Jandel Scientific, Chicago Il). Data are expressed as arithmetic mean \pm standard deviation unless otherwise indicated.

\section{Results}

We assessed the intestinal anti-viral humoral immune response following enteric reovirus infection in aged (18-24 months) and young ( 8 weeks) Balb/c mice. 10 days after oral infection with reovirus, anti-reovirus IgA and total IgA producing cells were enumerated in PP and spleens of aged and young mice by ELISPOT assay (Table 1). Aged mice had a significantly elevated frequency of PP anti-reovirus IgA antibody forming cells (AFC) as a fraction of total PP cells or total IgA AFC compared to young mice ( $p<0.008$ and $p<0.0005$ respectively). By contrast, the frequency of reovirus-specific IgA AFC among total cells or as a fraction of total IgA AFC in the spleens of aged and young Balb/c mice was not different $(p=0.277$ and $p=0.329$, respectively). The absolute numbers of PP cells recovered from aged and young animals were not significantly different $\left(1.07 \pm 0.37 \times 10^{7}\right.$ and $1.35 \pm 0.33 \times 10^{7}$ respectively, $\left.p=0.07\right)$. Small intestinal LP fragment cultures were established to assess the production of reovirusspecific IgA at the effector site of the intestinal mucosa. The quantity of reovirus-specific and total IgA in LP fragment culture supernatants of aged and young Balb/c mice was determined by ELISA (Figure 20). Aged mice showed a 3-fold greater production of reovirus-specific IgA compared to young mice ( $p=0.001$ ), whereas no significant difference was found between aged and young mice in total IgA production $(p=0.452)$. High titers of reovirus-specific IgG were detected in the serum of orally infected aged and young Balb/c mice, and were not significantly different (data not shown). 
The effect of ageing on the generation of reovirus-specific CTL following enteric reovirus infection was also determined. Splenocytes from aged and young Balb/c mice infected orally with reovirus 10 days previously were assessed for pCTL frequency by LDA (Figure 21A). Aged Balb/c mice had a frequency of $151 \pm 44$ pCTLs $/ 10^{6} \mathrm{CD}^{+}$splenocytes, which was not significantly different from the frequency of $120 \pm 57$ pCTLs $/ 10^{6} \mathrm{CD} 8^{+}$splenocytes from young Balb/c mice $(p=0.670)$. Absolute numbers of splenocytes were not different between aged and young mice, with $8.15 \pm 2.79 \times 10^{7}$ and $10.3 \pm 3.6 \times 10^{7}$ cells recovered per mouse, respectively $(p=0.077)$. Absolute numbers of $\mathrm{CD}^{+}$cells were also similar in aged and young Balb/c mice (data not shown). Additionally, bulk cultures of reovirus re-stimulated splenocytes from aged and young Balb/c mice showed no differences in anti-reovirus cytotoxicity of reovirus-infected murine KD2SV fibroblasts by bulk culture ${ }^{51} \mathrm{Cr}$ release assay (Figure 21B). Splenocytes from aged and young B6C3F1 mice infected orally with reovirus were also assessed for pCTL frequency by LDA, and for bulk culture cytotoxicity by ${ }^{51} \mathrm{Cr}$ release assay. Aged B6C3F1 mice had a frequency of $92.7 \pm 34.1$ pCTLs $/ 10^{6} \mathrm{CD} 8^{+}$splenocytes, which was similar to the frequency of $112 \pm 37.7 \mathrm{pCTLs} / 10^{6} \mathrm{CD}^{+}$splenocytes from young mice, $(p=0.471)$ (Figure 21C). Absolute numbers of splenocytes were not different between aged and young mice, with $3.55 \pm 0.84 \times 10^{7}$ and $3.89 \pm 1.43 \times 10^{7}$ cells per mouse, respectively $(p=0.90)$. Absolute numbers of $\mathrm{CD} 8^{+}$cells were also not found to be different between aged and young B6C3F1 mice $(p=0.06)$. Analysis of B6C3F1 splenic bulk cultures by ${ }^{51} \mathrm{Cr}$ release assay showed no difference in reovirus-specific cytotoxicity between aged and young mice (Figure 21D). To examine CTL generation at the intestinal inductive site, PP lymphocytes from orally infected aged and young B6C3F1were re-stimulated in vitro and assessed for CTL activity by ${ }^{51} \mathrm{Cr}$ release 
assay. CTL from aged and young mice mediated similar levels of virus-specific cytotoxicity over a range of effector to target ratios (Figure 22).

We assessed the systemic anti-viral humoral immune response following subcutaneous reovirus infection in aged (22 months) and young ( 8 weeks) Balb/c mice. 10 days after infection with reovirus, splenic reovirus-specific IgG AFC and total Ig AFC were enumerated for aged and young mice by ELISPOT assay (Figure 23). Aged mice had a similar frequency of reovirusspecific IgG AFC as a fraction of total Ig AFC compared to young mice $(66,419 \pm 18441 \mathrm{IgG}$ $\mathrm{AFC} / 10^{6} \mathrm{Ig} \mathrm{AFC}$ and $72,640 \pm 16,892 \mathrm{IgG} \mathrm{AFC} / 10^{6} \mathrm{Ig} \mathrm{AFC}$, respectively, $\left.p=0.637\right)$. Subcutaneously infected aged and young B6C3F1 mice also had similar frequencies of splenic reovirus-specific IgG AFC as a fraction of $\operatorname{Ig} \mathrm{AFC}\left(98,267 \pm 22,269 \mathrm{IgG} \mathrm{AFC} / 10^{6} \mathrm{Ig} \mathrm{AFC}\right.$ and $121,131 \pm 24,872 \operatorname{IgG} \mathrm{AFC} / 10^{6} \mathrm{Ig} \mathrm{AFC}$, respectively, $p=0.220$ ). The absolute numbers of splenic mononuclear cells recovered from aged and young animals were not significantly different for Balb/c mice $\left(17.12 \pm 3.90 \times 10^{7}\right.$ and $13.62 \pm 5.36 \times 10^{7}$ respectively, $\left.p=0.332\right)$ or B6C3F1 mice $\left(10.5 \pm 1.96 \times 10^{7}\right.$ and $8.52 \pm 1.91 \times 10^{7}$ respectively, $\left.p=0.198\right)$. Furthermore, similar titers of reovirus-specific $\operatorname{IgG}$ were detected in the serum of subcutaneously infected aged and young Balb/c mice $(p=0.110)$ and $\mathrm{B} 6 \mathrm{C} 3 \mathrm{~F} 1$ mice $(p=0.315)$ (Figure 24). We also assessed the generation of reovirus specific CTL in subcutaneously infected aged and young Balb/c mice and B6C3F1 mice. Bulk cultures of reovirus re-stimulated splenocytes from aged and young mice mediated similar levels of reovirus-specific cytotoxicity against reovirus-infected targets (data not shown).

As a previous report by Koga et al., (2000) showed diminished intestinal humoral responses by aged C57BL/6 mice to CT, we assessed intestinal CT specific humoral immune response following oral $\mathrm{CT}$ immunization in aged and young $\mathrm{B} 6 \mathrm{C} 3 \mathrm{~F} 1$ and Balb/c. Mice were 
orally immunized 3 times at 7 day intervals with CT. Seven days following the final immunization, CT-specific IgA AFC were enumerated in PP and spleens of aged and young mice by ELISPOT assay. Aged Balb/c mice had a significantly lower frequency of PP CTspecific IgA AFC as a fraction of total PP Ig AFC compared to young mice $(4,329.8 \pm 2,007.9$ $\operatorname{IgA~AFC~} / 10^{6} \operatorname{Ig} \mathrm{AFC}$ and 9,945.9 $\pm 1,276.0 \mathrm{IgA} \mathrm{AFC} / 10^{6} \mathrm{Ig} \mathrm{AFC}$, respectively, $p=0.015$ ) (Figure 25A). Similarly, aged B6C3F1 mice showed diminished numbers of PP CT-specific IgA $\mathrm{AFC}$ as a fraction of total PP cells compared to young mice $\left(2.8 \pm 2.0 \mathrm{IgA} \mathrm{AFC} / 10^{6} \mathrm{PP}\right.$ cells and $19.0 \pm 16.6 / 10^{6} \mathrm{PP}$ cells, respectively, $p=0.170$ ) (Figure 25B) as well as significantly fewer splenic CT-specific IgA AFC both absolutely (294 \pm 83 IgA AFC/ spleen and 33,875 \pm 11650 $\mathrm{IgA} \mathrm{AFC/} \mathrm{spleen,} \mathrm{respectively,} p=0.008)$ as a fraction of total splenic IgA AFC compared to young mice $\left(1,797.6 \pm 1,129.5 \mathrm{IgA} \mathrm{AFC} / 10^{6} \operatorname{Ig} \mathrm{AFC}\right.$ and $165,519.6 \pm 66,235.8 \mathrm{IgA} \mathrm{AFC} / 10^{6} \mathrm{Ig}$ AFC, respectively, $p=0.013)$.

Small intestinal LP fragment cultures were established to assess the production of CTspecific IgA at the intestinal mucosa. The quantity of CT specific IgA in LP fragment culture supernatants of aged and young Balb/c mice was determined by ELISA (Figure 26A). Aged Balb/c mice showed a 4-fold lower production of CT-specific IgA compared to young mice ( 8.3 $\pm 6.7 \mathrm{ng} / \mathrm{ml}$ and $36.3 \pm 35.1 \mathrm{ng} / \mathrm{ml}$, respectively, $p=0.246$ ). Similarly, fragment cultures from aged B6C3F1 mice produced 3-fold less CT specific IgA than those from young mice $(4.4 \pm 1.8$ $\mathrm{nr} / \mathrm{ml}$ and $13.2 \pm 7.8$, respectively, $p=0.130$ ). Extracts from fecal pellets formed 7 days following the final immunization were assessed for CT specific IgA content (Figure 26B). Aged Balb/c mice produced approximately 5 - fold less fecal CT specific IgA than young mice $(29.0 \pm 29.8$ $\mathrm{ng} / \mathrm{ml}$ extract and $148.8 \pm 38.8 \mathrm{ng} / \mathrm{ml}$ extract, respectively, $p=0.013)$. Similarly, aged B6C3F1 mice produced approximately 8 -fold less CT specific fecal $\operatorname{IgA}$ than young mice $(30.4 \pm 16.0$ 
$\mathrm{ng} / \mathrm{ml}$ and $262.7 \pm 276.1 \mathrm{ng} / \mathrm{ml}$ feces, respectively, $p=0.220)$. Additionally, aged Balb/c and B6C3F1 mice produced 4 to 2 times lower titers of serum CT specific IgG compared to young mice (data not shown). The effect of ageing on CTL memory responses was also examined. Mice infected orally with reovirus 16 months previously and subsequently orally re-infected or not reinfected were assessed for reovirus-specific splenic pCTL frequency by LDA and for bulk culture cytotoxicity by ${ }^{51} \mathrm{Cr}$ release assay. Re-infected mice had a pCTL frequency of $101 \pm$ $56.1 / 10^{6} \mathrm{CD}^{+}$cells in two separate experiments (Figure 27). The frequency of reovirus-specific splenic pCTL in mice infected once 16 months previously was barely detectable by LDA (5.6 \pm $5.2 / 10^{6} \mathrm{CD}^{+}$cells).

Mice infected only once 16 months previously had reovirus-specific IgA and IgGproducing cells within the bone marrow, and a variably lower frequency of such cells in the spleen. Mice re-infected with reovirus had a 5-6-fold higher frequency of reovirus-specific IgA-

producing cells and an approximately 2-fold higher frequency of IgG-producing cells in the bone marrow and spleen than mice infected only once 16 months previously (Table 2). Reovirusspecific serum IgG and LP culture supernatant IgA content of were also determined. Animals not re-infected with reovirus had nearly undetectable amounts of reovirus-specific IgA in PP and LP culture supernatants (Table 3), and similarly showed low titers of reovirus-specific IgG in the serum (data not shown).

\section{Discussion}

We used oral infection with reovirus to test the ability of the intestinal immune system of aged mice to respond to an enteric virus infection. Previous reports indicate that the immune system in general is compromised in ageing, and this has suggested a causal link between diminished immune function in ageing, with an increased incidence of vaccination failure, 
infectious disease, autoimmunity, and neoplasia. We found a similar splenic anti-reovirus IgG response and an enhanced anti-reovirus intestinal IgA immune response in the PP of aged Balb/c mice, and an equivalent to slightly enhanced response in $\mathrm{B} 6 \mathrm{C} 3 \mathrm{~F} 1$ mice, compared to young mice. The enhanced IgA response in the priming site of the PP and the enhanced secretion of reovirus-specific IgA within the small intestine LP of aged animals together suggest that the primed reovirus-specific IgA-producing cells proliferated and trafficked normally to the LP in the aged animals. By contrast, migration to the spleen of reovirus-specific IgA-producing cells was similar in both aged and young mice. The apparent increase in IgA humoral immunity to intestinal virus infection was not a compensatory effect of diminished virus-specific $\mathrm{T}$ cellmediated cytotoxicity, as the splenic reovirus-specific pCTL frequency and bulk culture cytotoxicity of lymphocytes from aged animals was not different from that of young animals.

In addition to mediating cytotoxicity, enteric $\mathrm{CD} 8^{+} \mathrm{T}$ cells have been suggested to exert a modulatory effect upon intestinal humoral immune responses. Kawanishi et al. (34) found that unfractionated mycobacterial antigens given orally to aged mice resulted in an enhanced specific IgA response compared to that of young mice. This elevated response was attenuated by the exogenous addition of IL-2, which caused the IgA response of the aged mice to resemble that of the young. $\mathrm{CD} 8^{+} \mathrm{T}$ cells have also been shown to have a modulatory role in the intestinal humoral immune response to reovirus. Major and Cuff (46) showed that $\beta 2$-microglobulin deficient mice, which lack conventional $\mathrm{CD}^{+} \mathrm{T}$ cells, mount an enhanced anti-viral IgA response following enteric reovirus infection as compared to wild-type controls. In light of these reports, our data suggest a functional preservation in the cytotoxic function of virus-specific $\mathrm{CD}^{+} \mathrm{T}$ cells with ageing, despite a potential diminution in regulatory function exerted on the intestinal humoral immune response. 
Bulk cultures of PP cells from aged and young $\mathrm{B} 6 \mathrm{C} 3 \mathrm{~F} 1$ mice re-stimulated in vitro showed similar levels of reovirus-specific cytotoxicity. Taken together with the similar reovirusspecific pCTL frequencies recovered from the spleens of aged and young mice, these results suggest that $\mathrm{pCTL}$ priming within the PP and migration to the spleen occurs normally in aged mice. Following oral reovirus infection, reovirus-specific CTLs localize to the PP (38), spleen (47), and the effector site of the intestinal epithelial surface (48). These $\operatorname{CD} 8^{+} \mathrm{T}$ cell receptor $\alpha \beta^{+}$ cells may be initially primed within the PP $(49,50)$, and would therefore be of similar origin to those CTLs we have assayed within the spleen and PP. Thus, similar priming of virus-specific CTL responses in the PP and intact pCTL migration indicated by similar frequencies of $\mathrm{pCTL}$ recovered from the spleens together suggest similar numbers of virus-specific CTL migrating to and mediating cytotoxicity within the gut mucosal effector sites of the LP and intestinal epithelium. Therefore, it appears that in both strains of mice tested the priming and cytotoxic effector functions of reovirus-specific CTL in the intestinal mucosa are intact in aged animals compared to young. Furthermore, the mucosal humoral immune response to virus infection is intact, or even enhanced, in agreement with the results of Kawanishi et al. (34).

Several factors may play a role in attenuating the immunosenescence of the gut immune system. The intestinal intraepithelial cells and resident LP mononuclear cells (51) produce a variety of immunomodulatory factors as a result of constant stimulation by dietary and microfloral antigens. Local production of IL-1, IL-6, IL-7, IL-8, IL-12, IL-15, IL-16, IL-18 (5257), and innate mucosal adjuvants such as lymphotactin (58) may maintain a high degree of constant immune tension that potentiates immune responsiveness to reovirus.

In contrast to immune responses induced by enteric reovirus infection, our finding of diminished intestinal and systemic humoral immunity in aged Balb/c and B6C3F1 mice 
following oral immunization with CT agrees well with the findings of Koga et al. (37), wherein mice one year of age or older showed diminished mucosal and systemic humoral and cellular immune responses to oral immunization with ovalbumin and CT compared to young controls. However, the inflammatory state induced by a replicating, invasive intestinal pathogen may account for the enhanced humoral and intact cellular immunity seen in aged mice following oral reovirus infection, which may not be equivalently mimicked with the classic mucosal adjuvant $\mathrm{CT}$, or an otherwise inert protein antigen such as ovalbumin. This prediction is further supported by our findings of similar humoral and CTL immune responses between aged and young Balb/c and $\mathrm{B} 6 \mathrm{C} 3 \mathrm{~F} 1$ mice following subcutaneous reovirus infection, and suggests innate immune factors can play an important role in overcoming otherwise defective initiation of adaptive immune responses in ageing.

Finally, we have assessed humoral and CTL immunity in aged mice infected orally with reovirus T1L early in life and re-infected orally 16 months later. Virus infection early in life generates memory CTL and B cells, some of which might persist into old age (33), giving some degree of a secondary immune response to re-infection of the aged animal despite systemic immunosenescence (59). Although mice infected orally with reovirus in youth had virus-specific IgG and IgA producing cells in the spleens and bone marrow, they had very low splenic reovirus-specific pCTL frequencies at 16 months post-infection.

Aged mice orally re-infected with reovirus mounted both mucosal and systemic humoral immune responses, and generated splenic pCTL. It is, however, uncertain to what degree these responses reflect the re-activation of memory B cells and $\mathrm{pCTL}$, as opposed to a primary response of naïve pCTL and B cells. In any event, oral reovirus infection of aged mice induced both humoral and cell mediated immunity. 
In conclusion, we have shown the maintenance during ageing of the priming and function of both humoral and cellular intestinal immunity following enteric virus infection. We have also noted enhanced mucosal humoral responsiveness, in agreement with the previous findings of Kawanishi et al. (34). However, instead of finding generally diminished $\mathrm{CD} 8^{+} \mathrm{T}$ cell function, we have noted the preservation of $\mathrm{CD} 8^{+} \mathrm{T}$ cell mediated cytotoxicity. It is possible that the loss of $\mathrm{CD}^{+} \mathrm{T}$ cell regulatory function on intestinal humoral immunity may be more susceptible to ageing than is CTL function. In any event, enhanced mucosal humoral immunity concurrent with preserved CTL function in the aged intestine need not be considered an ageing-related dysfunction, but may be an exploitable phenomenon in the design and application of immunotherapy strategies aimed at generating potent mucosal and systemic immunity in the elderly. 
Table I. Elispot analysis of PP and spleens from old and young Balb/c mice ${ }^{a}$

\begin{tabular}{ccccc} 
Tissue & group & reo-IgA $/ 10^{6}$ cells $^{b}$ & Total IgA $/ 10^{6}$ cells & reo-IgA $/ 10^{6} \operatorname{IgA}{ }^{c}$ \\
\hline \multirow{3}{*}{$\mathrm{PP}^{d}$} & aged & $92(52)$ & $1981(804)$ & $47354(18968)$ \\
& young & $22(19)$ & $3470(2370)$ & $12670(11379)$ \\
& $p$ & 0.008 & 0.058 & 0.0005 \\
\multirow{3}{*}{ Spleen } & aged- & $22(19)$ & $581(262)$ & $38525(25395)$ \\
& young & $17(9)$ & $554(340)$ & $32700(21279)$ \\
& $p$ & 0.277 & 0.431 & 0.329 \\
\hline
\end{tabular}

${ }^{a} \mathrm{n}=8$ aged, 7 young; data are compiled from 2 separate experiments.

${ }^{b}$ reovirus-specific IgA-producing cells $/ 10^{6}$ mononuclear cells

${ }^{c}$ reovirus-specific IgA-producing cells $/ 10^{6} \mathrm{IgA}$-producing cells

${ }^{d}$ Number of PP cells recovered from aged and young mice were comparable.

Aged mice yielded $1.07 \pm 0.37 \times 10^{7}$ cells/mouse. Young mice yielded $1.35 \pm 0.33 \times 10^{7}$ cells/mouse, $p=0.07$. 
Table II. Elispot analysis of bone marrow and spleens from old mice

$\begin{array}{lll}\text { Experiment } & 1 & 2\end{array}$

\begin{tabular}{|c|c|c|c|c|c|}
\hline Tissue & re-infection ${ }^{a}$ & reo- $\operatorname{IgA} / 10^{6}$ cells $^{b, c}$ & reo-IgG/ $10^{6}$ cells $^{c, d}$ & reo-IgA $/ 10^{6}$ cells $^{b, c}$ & reo-IgG/ $10^{6}$ cells $^{c, d}$ \\
\hline \multirow[t]{2}{*}{$\mathrm{BM}$} & - & $18(18)$ & $38(23)$ & $42(11)$ & $152(48)$ \\
\hline & + & $109(30)$ & $104(26)$ & $256(187)$ & $288(217)$ \\
\hline \multirow[t]{2}{*}{ Spleen } & - & $2(3)$ & $42(11)$ & $12(16)$ & $39(20)$ \\
\hline & + & $13(2)$ & $110(70)$ & $118(76)$ & $82(19)$ \\
\hline
\end{tabular}

${ }^{a}$ All mice were infected orally at 8 weeks of age and 16 months later were either re-infected $(+)$ or not re-infected (-).

${ }^{b}$ Mean \pm standard deviation

${ }^{c}$ reovirus-specific IgA-producing cells $/ 10^{6}$ cells

${ }^{D}$ reovirus-specific IgG-producing cells $/ 10^{6}$ cells 
Table III. Reovirus specific and total IgA from LP and PP fragment cultures from old mice

\begin{tabular}{|c|c|c|c|c|c|}
\hline \multicolumn{2}{|c|}{ Experiment } & \multicolumn{2}{|c|}{1} & \multicolumn{2}{|c|}{2} \\
\hline Tissue & re-infection ${ }^{a}$ & reo-IgA $(\mathrm{ng} / \mathrm{ml})^{b}$ & reo-IgG $(\mathrm{ng} / \mathrm{ml})^{b}$ & reo-IgA $(\mathrm{ng} / \mathrm{ml})^{b}$ & reo- $\operatorname{IgA}(\mathrm{ng} / \mathrm{ml})^{b}$ \\
\hline \multirow[t]{2}{*}{ LP } & - & $<4.0$ & $24319(2746)$ & $0.9(1.3)$ & $5550(2526)$ \\
\hline & + & $60.6(49.4)$ & 29477 (2948) & $31.3(17)$ & 7195 (2629) \\
\hline \multirow[t]{2}{*}{ PP } & - & $<0.8$ & $1262(48)$ & $<0.2$ & $1732(556)$ \\
\hline & + & $9.9(7.3)$ & $2673(1187)$ & $3.3(0.5)$ & $2978(1413)$ \\
\hline
\end{tabular}

${ }^{a}$ All mice were infected orally at 8 weeks of age and 16 months later were either re-infected $(+)$ or not re-infected (-).

${ }^{b}$ Mean \pm standard deviation 


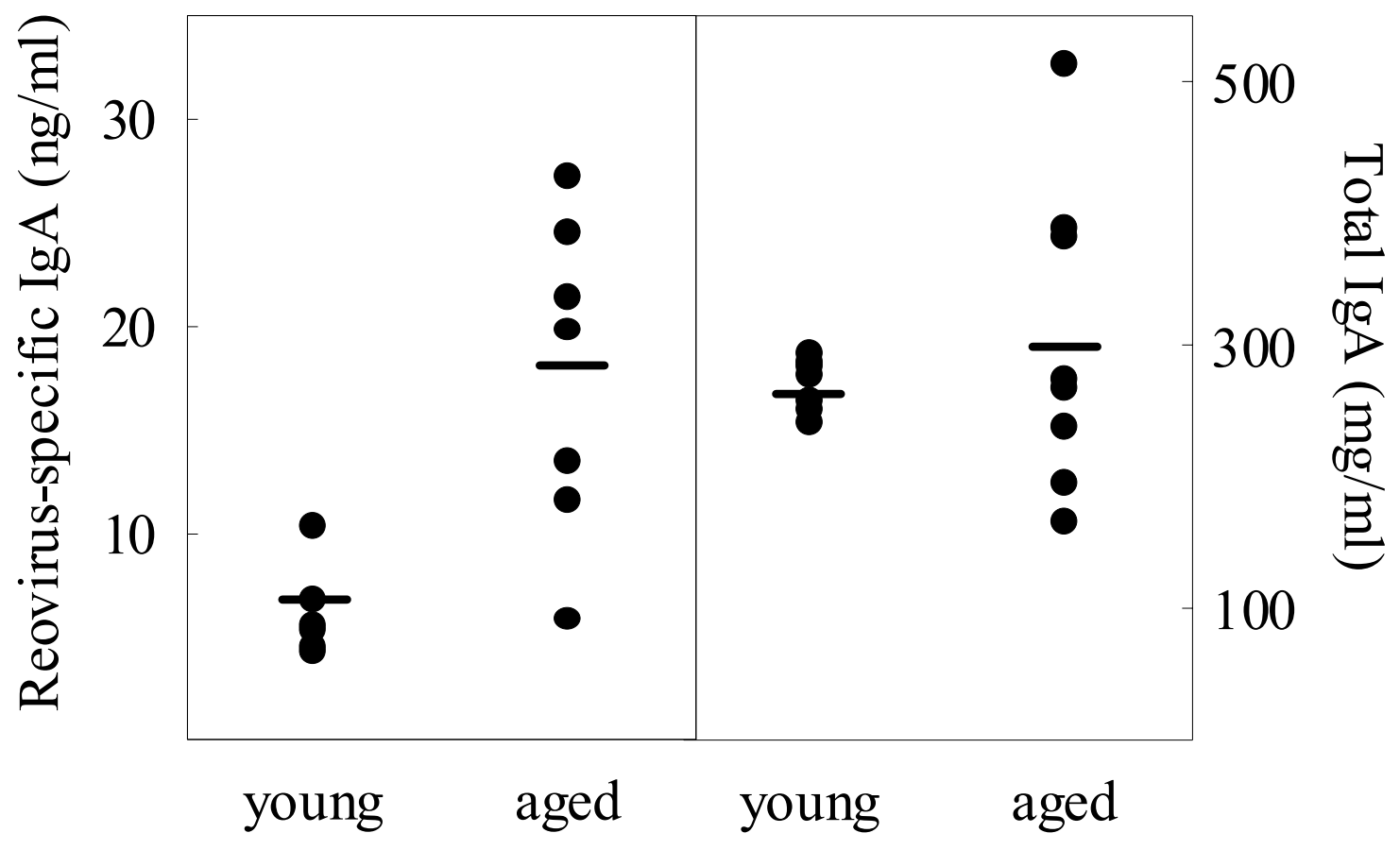


Figure 20. Reovirus-specific and total IgA in LP fragment cultures. Old and young Balb/c mice were infected orally with reovirus, and 10 days later small intestines were removed, fragmented, and cultured for 5 days as described in methods. Reovirus-specific and total IgA concentrations were determined for individual mice by ELISA. Data are expressed in $\mathrm{ng} / \mathrm{ml}$ for reovirus-specific IgA, and $\mu \mathrm{g} / \mathrm{ml}$ for total IgA, as determined by titration of supernatants in comparison to a known murine IgA standards. Reovirus-specific IgA concentration of LP culture supernatants from aged mice was found to be significantly elevated compared to LP culture supernatants from young mice $(p=0.001)$. No significant difference was found in total IgA concentration between old and young mice $(p=0.452)$. 

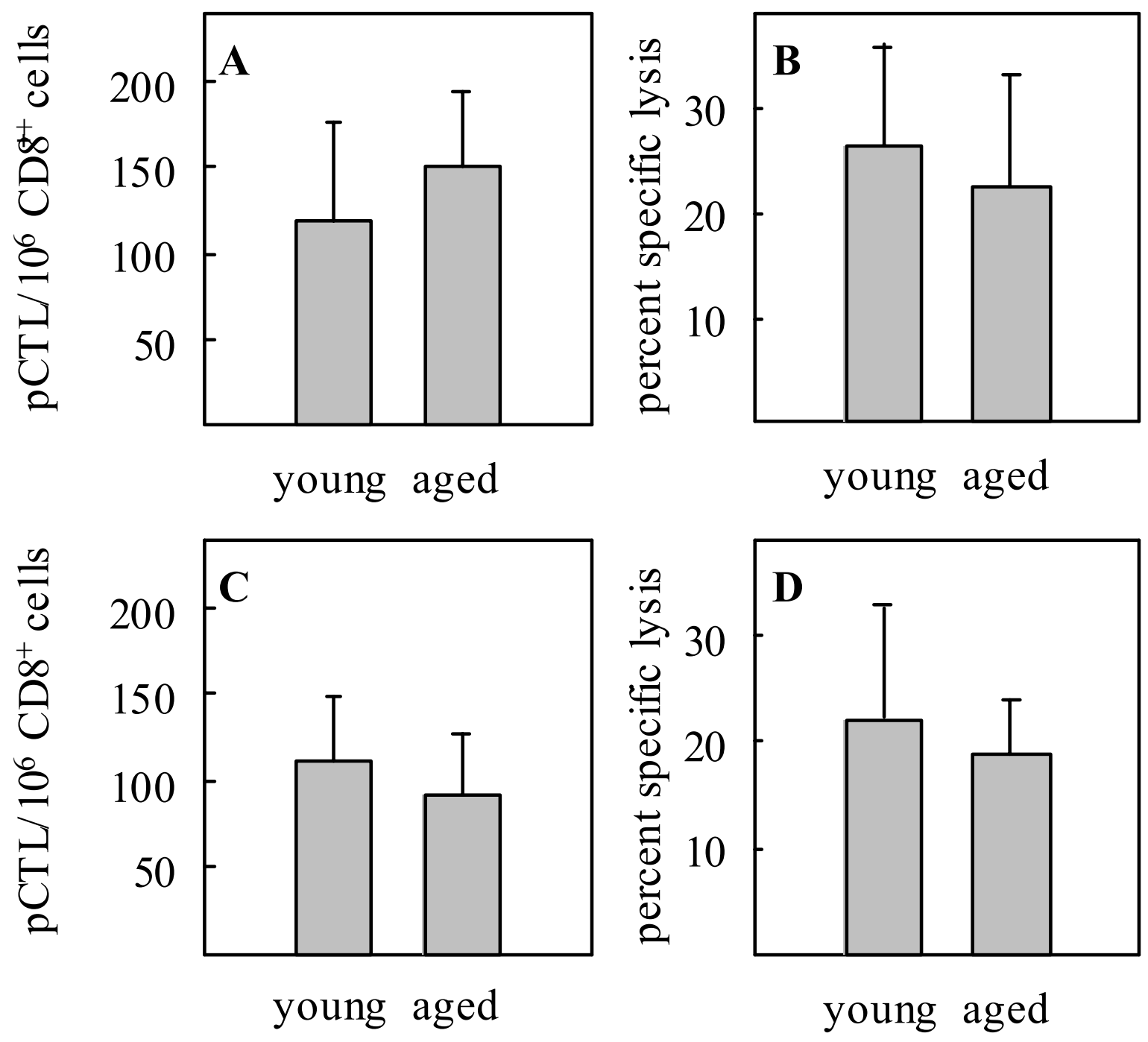
Figure 21. Splenic reovirus-specific pCTL frequencies of young and old mice. Balb/c (A) and $\mathrm{B} 6 \mathrm{C} 3 \mathrm{~F} 1(\mathrm{C})$ mice were infected orally with reovirus and 10 days later spleens were assessed for the frequency of pCTL by limiting dilution analysis as described in methods. Data are expressed as means \pm standard deviation $/ 10^{6} \mathrm{CD8}^{+}$cells.

CTL activity of bulk cultures of splenocytes from Balb/c (B) and B6C3F1 (D) mice were also similar.

Data are expressed as the means and standard deviations of cytotoxicity against reovirus-infected target cells less background non-specific lysis for triplicate wells at an effector: target ratio of 25:1 


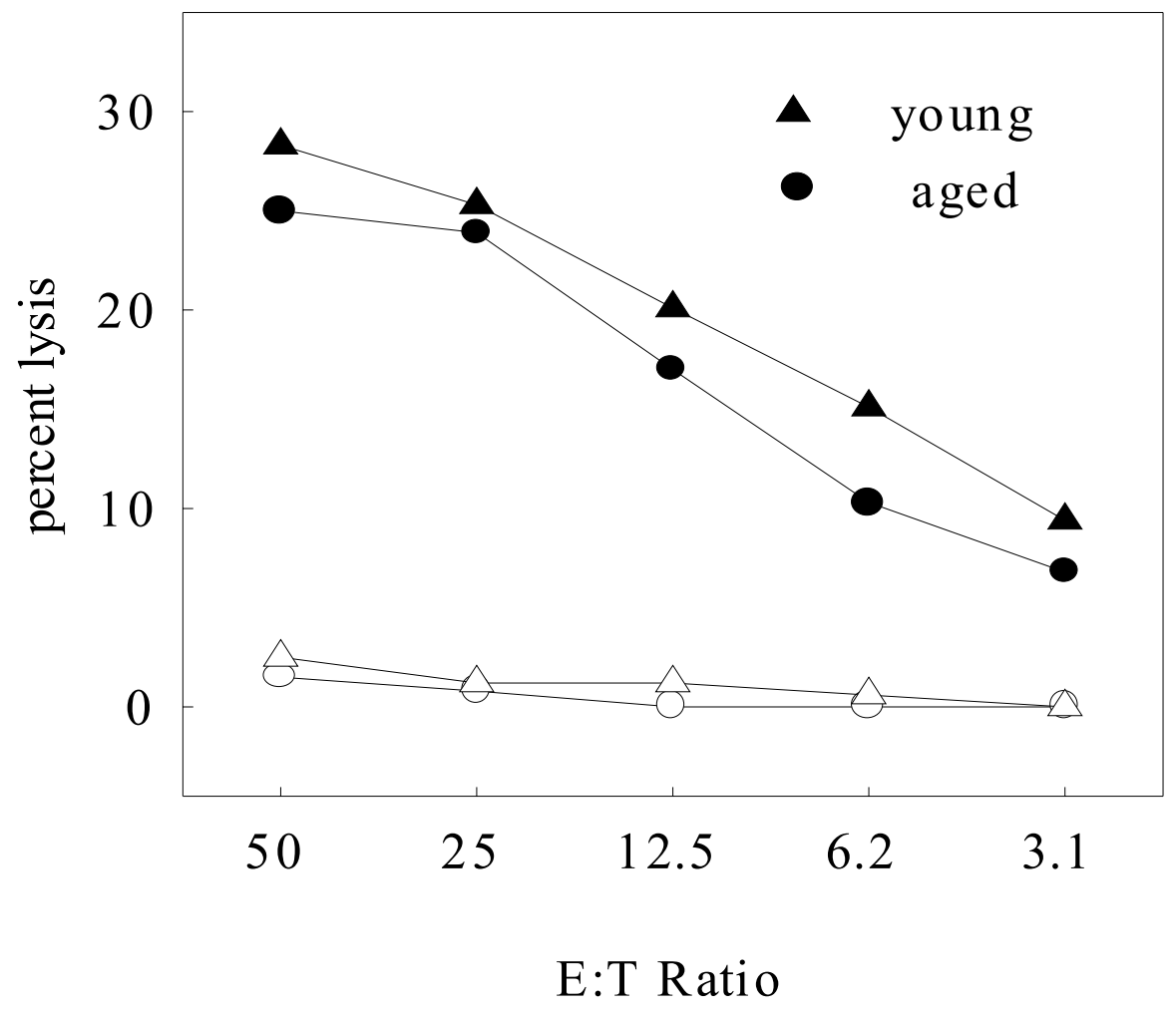


Figure 22. $51 \mathrm{Cr}$ release assay of pooled PP lymphocytes from orally infected young and old B6C3F1 mice re-stimulated in vitro with reovirus for 2 weeks. Data are expressed as the mean cytotoxicity against reovirus-infected ( $\triangle$ young PP, $\bullet$ old PP) and non-infected $(\triangle$ young PP, $\bigcirc$ old PP) targets. 

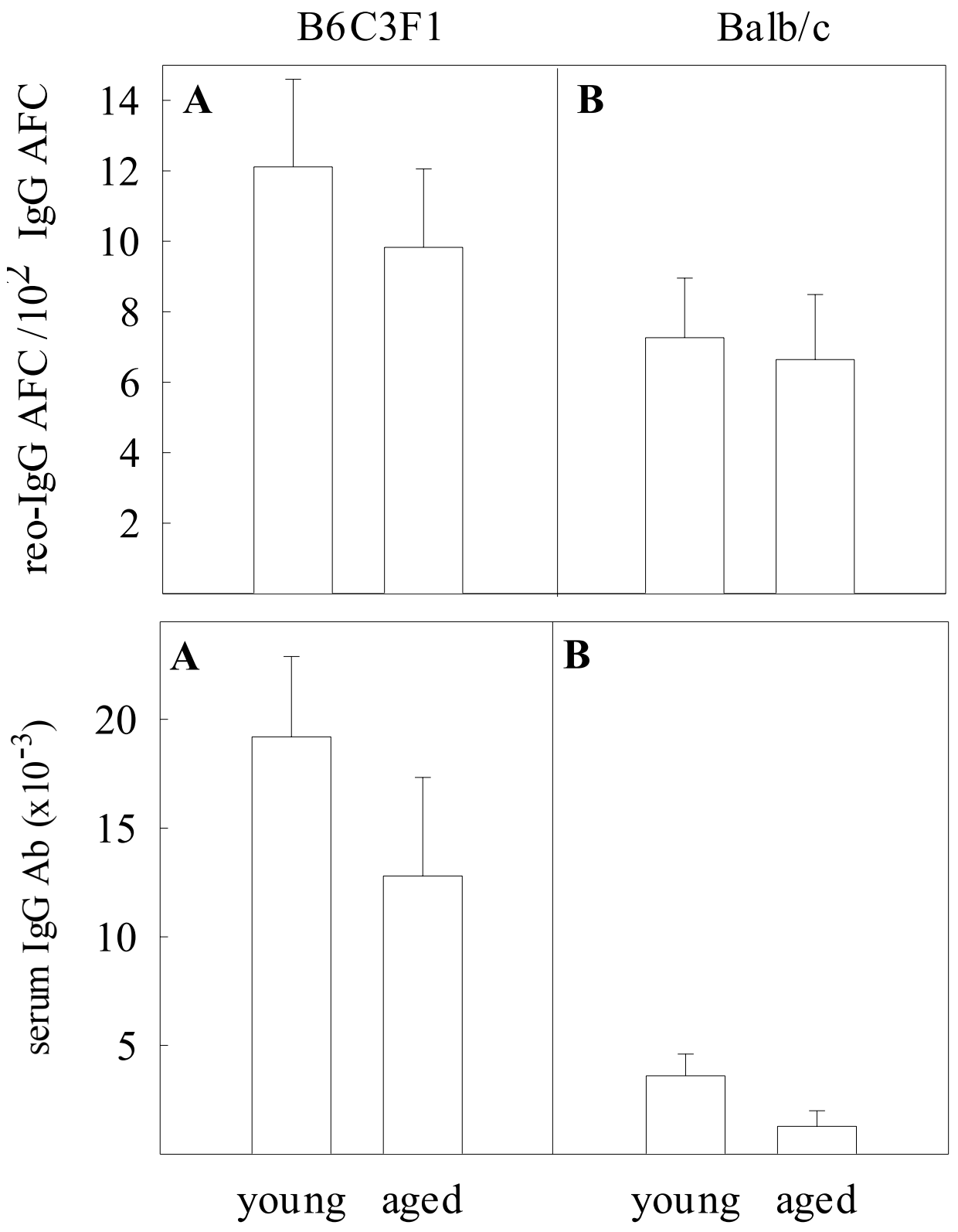
Figure 23. Splenic reovirus-specific IgG AFC frequencies of young and old mice. B6C3F1 (A) and Balb/c (B) mice were infected subcutaneously with reovirus and 10 days later spleens were assessed for the frequency of reovirus-specific IgG AFC by ELISPOT analysis as described in methods. Data are expressed as means \pm standard error IgG AFC / $10^{2}$ Ig AFC. 


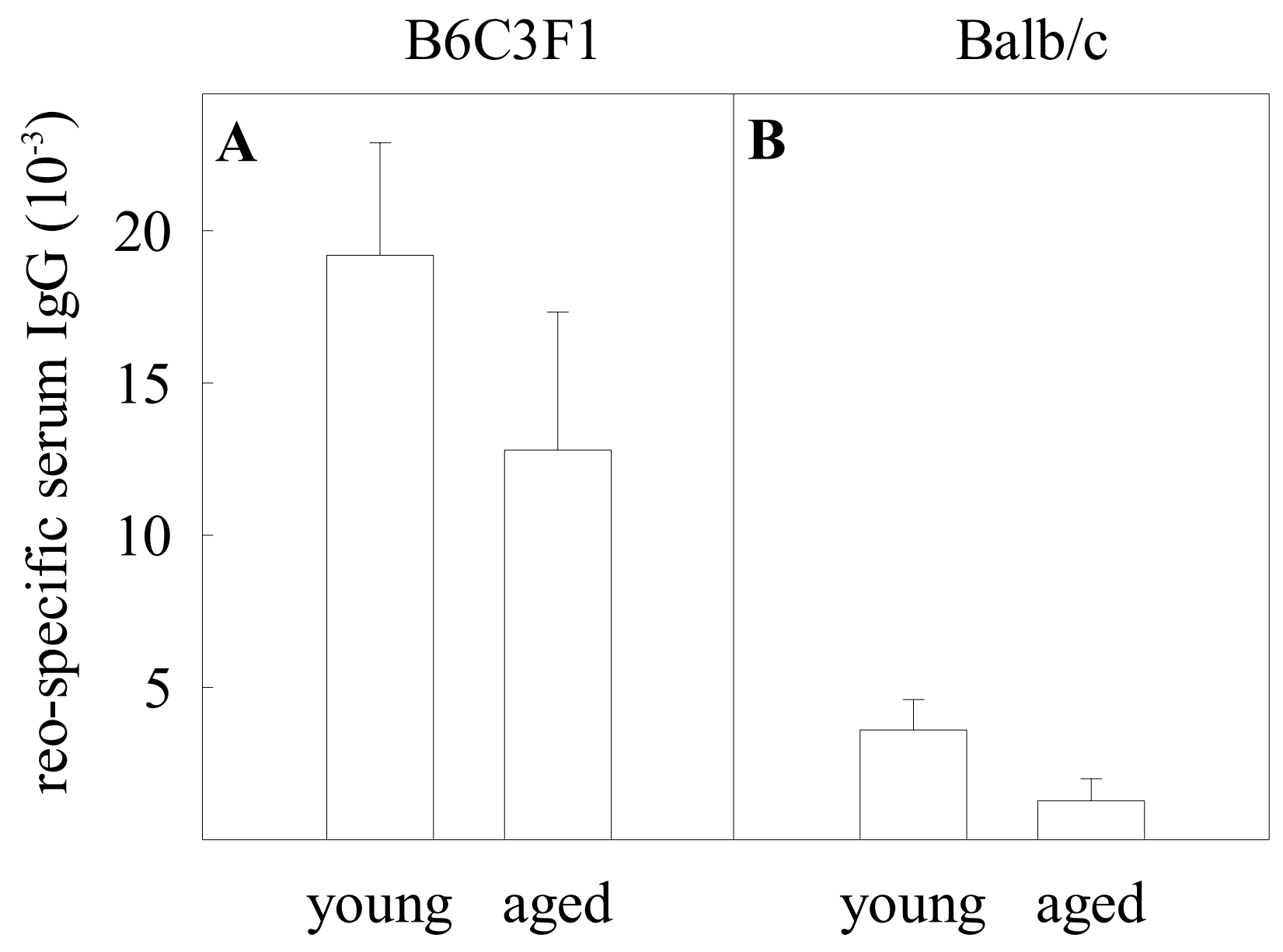


Figure 24. Reovirus-specific serum IgG titers from young and old mice. B6C3F1 (A) and Balb/c (B) mice were infected subcutaneously with reovirus and 10 days later sera were collected and assessed for reovirus-specific IgG by ELISA. Data are expressed as reciprocal mean titer \pm standard error IgG $\left(\times 10^{-3}\right)$. 


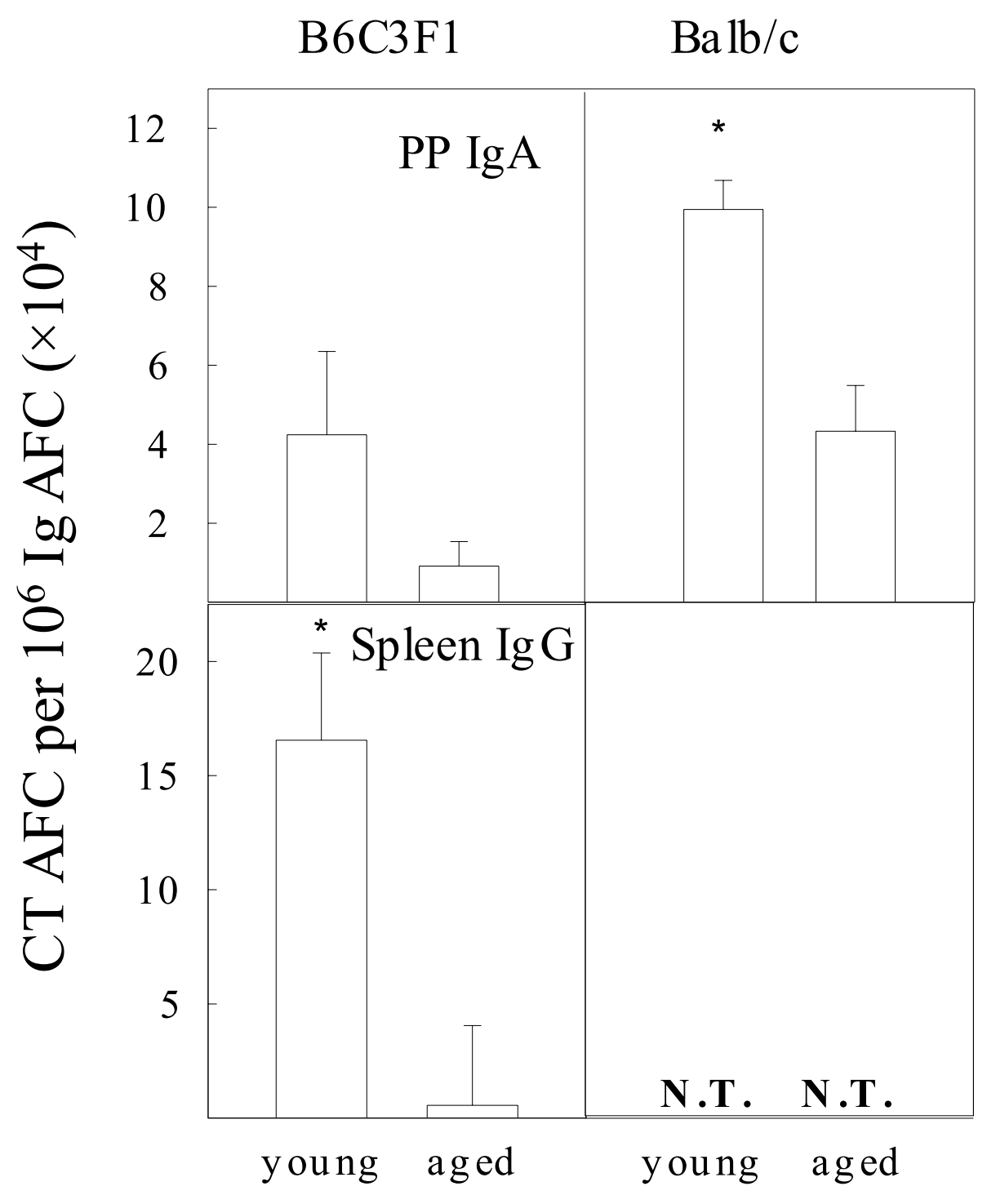


Figure 25. Peyer's patch and splenic CT-specific IgA AFC frequencies of young and old mice. B6C3F1 (A and C) and Balb/c (B) mice were immunized orally 3 times with CT and 7 days following the last immunization PP and spleens were assessed for the frequency of CT specific IgA AFC by ELISPOT analysis as described in methods. Data are expressed as means \pm standard error IgA or IgG AFC / $10^{2}$ Ig AFC. Young Balb/c mice had significantly more PP CT-specific IgA AFC than old mice $(p=0.015)$; young B6C3F1 mice had significantly more splenic CT-specific IgG AFC than old mice $(p=0.013)$. N.T.= not tested. 


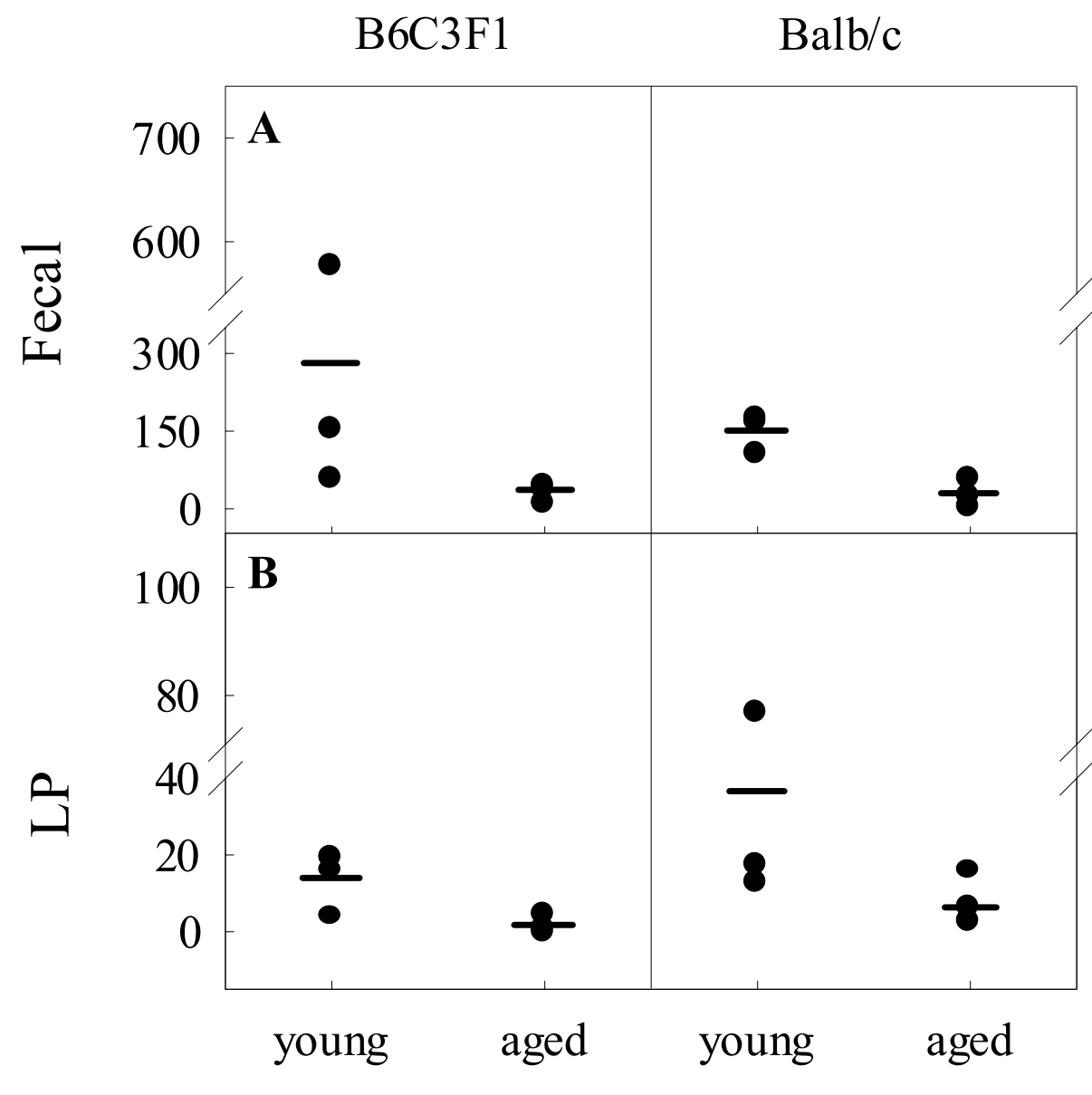


Figure 26. CT-specific IgA in fecal pellet extracts (A) and LP fragment cultures (B). Young and old B6C3F1 and Balb/c mice were orally immunized 3 times with CT, and 7 days following the final immunization, small intestines were removed, fragmented, and cultured for 5 days as described in methods. Additionally, fecal pellets formed on day seven following the final immunization were collected and extracted with PBS. CT-specific IgA concentrations were determined for individual mice by ELISA. Data are expressed in ng CT-specific IgA / ml supernatant and ng CT-specific IgA / ml fecal extract, as determined by titration of samples in comparison to a known murine IgA standard. 


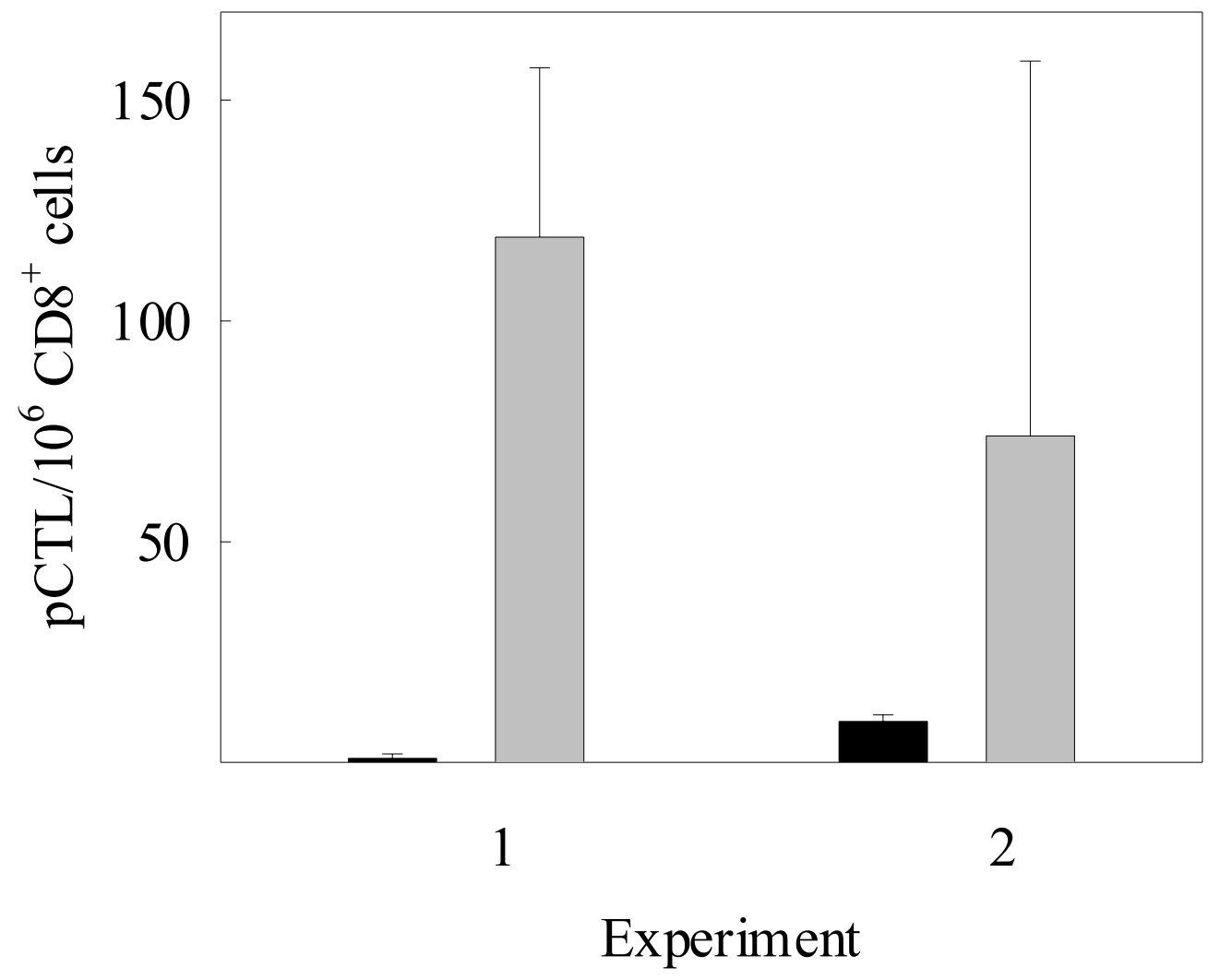


Figure 27. Splenic reovirus-specific pCTL frequencies of mice infected orally 16 months previously. Mice were either re-infected orally $(\square)$ or not re-infected ( $\square)$, and 10 days later spleens were assessed for the frequency of pCTL by limiting dilution analysis as described in methods. Data are expressed as (means \pm standard deviation $) /\left(10^{6} \mathrm{CD8}^{+}\right.$cells $)$. Reinfected groups consisted of $n=3$ and $n=2$ mice for experiments 1 and 2 , respectively. Not re-infected groups, $n=2$ and $n=3$ mice. 


\section{References}

Phair, J.P., Hsu, C.S. and Y.L. Hsu. 1988. Ageing and infection. Ciba Found. Symp. 134:143.

Chakravarti, B., and G.N. Abraham. 1999. Ageing and T cell-mediated immunity. Mech. Ageing Dev. 108:183.

Effros, R.B., and R.L.Walford. 1983. The immune response of aged mice to influenza: diminished T-cell proliferation, interleukin 2 production and cytotoxicity. Cell. Immunol. $81: 298$.

Bender, B.S., Taylor, S.F. Zander, D.S. and R. Cottey. 1995. Pulmonary immune responses of young and aged mice after influenza challenge. J. Lab. Clin. Med. 126:169.

Fagiolo, U., Amadori, A. Cozzi, E. Bendo, R. Lama, M. Douglas, A. and G. Palu. 1993. Humoral and cellular immune response to influenza virus vaccination in aged humans. Ageing. $5: 451$.

Mbawuike, I.N., Lange, A.R. and R.B. Couch. 1993. Diminished influenza A virus-specific MHC class I-restricted cytotoxic T lymphocyte activity among elderly persons. Viral Immunol. $6: 55$

Mbawuike, I.N., Acuna, C.L. Walz, K.C. Atmar, R.L. Greenberg, S.B. and R.B. Couch. 1997. Cytokines and impaired CD8+ CTL activity among elderly persons and the enhancing effect of IL-12. Mech. Ageing Dev. 94:25. 
Bender, B.S., Johnson, M.P. and P.A. Small. 1991. Influenza in senescent mice: impaired cytotoxic T-lymphocyte activity is correlated with prolonged infection. Immunology 72:514.

Taylor, S.F., Cottey, R.J. Zander, D.S. and B.S. Bender.1997. Influenza infection of beta 2microglobulin deficient (beta $2 \mathrm{~m}-/-$ ) mice reveals a loss of CD4+ T cell functions with ageing. $J$. Immunol. 159:3453.

Effros, R.B. and R.L. Walford. 1984.The effect of age on the antigen presenting mechanism in limiting dilution precursor cell frequency analysis. Cell. Immunol. 88:531.

Whisler, R.L., Bagenstose, S.E. Newhouse, Y.G. and K.W. Carle. 1997. Expression and catalytic activities of protein tyrosine kinases (PTKs) Fyn and Lck in peripheral blood T cells from elderly humans stimulated through the T cell receptor (TCR)/CD3 complex. Mech. Ageing Dev. 98:57.

Fulop, T., Gagne, D. Goulet, A.C. Desgeorges, S. Lacombe, G. Arcand, M. and G. Dupuis. 1999. Age-related impairment of p561ck and ZAP-70 activities in human T lymphocytes activated through the TcR/CD3 complex. Exp. Gerontol. 34:197.

Whisler, R.L., Chen, M. Liu, B. and Y.G. Newhouse. 1999. Age-related impairments in TCR/CD3 activation of Zap-70 are associated with reduced tyrosine phosphorylations of zetachains and p59fyn/p561ck in human T cells. Mech. Ageing Dev. 111:49.

Engwerda, C.R., Handwerger, B.S. and B.S. Fox. 1994. Aged T cells are hyporesponsive to costimulation mediated by CD28. J. Immunol. 152:3470. 
Bloom, E.T., Umehara, H. Bleackley, R.C. Okumura, K. Mostowski, H. and J.T. Babbitt. 1990. Age-related decrement in cytotoxic T lymphocyte (CTL) activity is associated with decreased levels of mRNA encoded by two CTL-associated serine esterase genes and the perforin gene in mice. Eur. J. Immunol. 20:2309.

Horvath, J.A., Mostowki, H. S. Okumura, K. and E.T. Bloom. 1992. Pore-forming protein in individual cytotoxic $\mathrm{T}$ lymphocytes: the effect of senescence provides a probe for understanding the lytic mechanism. Eur. J. Immunol. 22:2649.

Rukavina, D., Laskarin, G. Rubesa, G. Strbo, N. Bedenicki, I. Manestar, D. Glavas, M. Christmas, S.E. and Podack, E.R. 1998. Age-related decline of perforin expression in human cytotoxic T lymphocytes and natural killer cells. Blood. 92:2410.

Mariani, E., Sgobbi, S. Meneghetti, A. Tadolini, M. Tarozzi, A. Sinoppi, M. Cattini, L. and A. Facchini. 1996. Perforins in human cytolytic cells: the effect of age. Mech. Ageing Dev. 92:195. Nordin, A.A., and G.D. Collins. 1983. Limiting dilution analysis of alloreactive cytotoxic precursor cells in ageing mice. J. Immunol. 131:2215.

Zharhary, D. Segev, Y. and H.E. 1984. Gershon. T-cell cytotoxicity and ageing: differing causes of reduced response in individual mice. Mech. Ageing Dev. 25:129.

Mariani, E., Roda, P. Mariani, A.R. Vitale, M. Degrassi, A. Papa, S. and A. Facchini. 1990. Ageassociated changes in CD8+ and CD16+ cell reactivity: clonal analysis. Clin. Exp. Immunol. 81:479. 
Fagnoni, F.F., Vescovini, R. Passeri, G. Bologna, G. Pedrazzoni, M. Lavagetto, G. Casti, A. Franceschi, C. Passeri, M. and P. Sansoni. 2000. Shortage of circulating naïve CD8 (+) T cells provides new insights on immunodeficiency in ageing. Blood. 95:2860.

Vissinga, C., Hertogh-Huijbregts, A. Rozing, J. and L. Nagelkerken. 1990. Analysis of the agerelated decline in alloreactivity of CD4+ and CD8+ T cells in CBA/RIJ mice. Mech. Ageing Dev. 51:179.

Miller, R.A. 1984. Age-associated decline in precursor frequency for different T cell-mediated reactions, with preservation of helper or cytotoxic effect per precursor cell. J. Immunol. 132:63.

Mbawuike, I.N., Acuna, C. Caballero, D. Pham-Nguyen, K. Gilbert, B. Petribon, P. and M. Harmon. 1996. Reversal of age-related deficient influenza virus-specific CTL responses and IFN-gamma production by monophosphoryl lipid A. Cell Immunol. 173:64.

Zhang, Y., Acuna, C.L. Switzer, K.C. Song, L. Sayers, M. and I.N. Mbaiwuike. 2000. Corrective effects of interleukin-12 on age-related deficiencies in IFN-gamma production and IL-12Rbeta2 expression in virus-specific CD8+ T cells. J. Interferon Cytokine Res. 20:235. Schmucker, D.L., Daniels, C.K. Wang, R.K. and K. Smith. 1988. Mucosal immune response to cholera toxin in ageing rats. I. Antibody and antibody-containing cell response. Immunology. 64:691. 
Jones, S.E., Davila, D.R. Haley, P.J. and D.E. Bice. 1993. The effects of age on immune responses in the antigen-instilled dog lung. Antibody responses in the lung and lymphoid tissues following primary and secondary antigen instillation. Mech. Ageing Dev. 68:191.

Zharhary, D. 1986.T cell involvement in the decrease of antigen-responsive B cells in aged mice. Eur. J. Immunol. 16:1175.

Marcenaro, L., Russo, C. Kim, Y.T. Siskind, J.W. and M.E. Weksler. 1989. Immunological studies of ageing. Normal B-cell repertoire in aged mice: studies at a clonal level. Cell Immunol. 119:202.

Nagarkatti, P.S. Seth, A. Nagarkatti, M. Muthusamy, N. Rychlik, B. and B.A. Subbarao. 1989. Specific defect in the proliferative capacity of B cells from old mice stimulated with autoreactive T cells. Cell Immunol. 120:102.

Subbarao, B., Morris, J. and R.J. Kryscio. 1990. Phenotypic and functional properties of Blymphocytes from aged mice. Mech. Ageing Dev. 51:223.

Gallichan, W.S., and K.L.Rosenthal. 1996. Long-lived cytotoxic T lymphocyte memory in mucosal tissues after mucosal but not systemic immunization. J. Exp. Med. 184:1879. Kawanishi, H., Ajitus, S. and S. Mirabella. 1990. Impaired humoral immune responses to mycobacaterial antigen in aged murine gut-associated lymphoid tissues. Mech. Ageing Dev. $54: 143$. 
Ajitsu, A., Mirabella, S. and H. Kawanishi. 1990. In vivo immunologic intervention in agerelated T cell defects in murine gut-associated lymphoid tissue by Il-2. Mech. Ageing Dev. 54:163.

Thoman, M.L., and W.O. Weigle. 1985. Reconstitution of in vivo cell-mediated lympholysis responses in aged mice with interleukin-2. J Immunol. 134:949.

Koga, T., McGhee, J.R. Kato, H. Kato, R. Kiyono, H. and K. Fujihashi. 2000. Evidence for early ageing in the mucosal immune system. J. Immunol. 165:5352.

London, S.D., Rubin, D.H. and J.J. Cebra. 1987. Gut mucosal immunization with reovirus serotype 1/L stimulates virus-specific cytotoxic $\mathrm{T}$ cell precursors as well as IgA memory cells in Peyer's patches. J. Exp. Med. 165:830.

Smith, R.E., Zweerink, H.J., and W.K. Joklik. 1969. Polypeptide components of virions, top component and cores of reovirus type 3. Virology. 39:791.

Cuff, C.F., Lavi, E. Cebra, C.K. Cebra, J.J. and D.H. Rubin. 1990. Passive immunity to fatal reovirus serotype 3 -induced meningoencephalitis mediated by both secretory and transplacental factors in neonatal mice. J. Virol. 64:1256.

Cuff, C.F., Fulton, J.R. Barnett, J.B. and C.S. Boyce. 1998. Enteric reovirus infection as a probe to study immunotoxocity of the gastrointestinal tract. Toxicol. Sci. 42:99. 
Taswell, C. 1981.Limiting dilution assays for the determination of immunocompetent cell frequencies. I. Data analysis. J. Immunol. 126:1614.

Major, A.S., and C.F. Cuff. 1996. Effects of the route of infection on immunoglobulin G subclasses and specificity of the reovirus-specific humoral immune response. J. Virol. 70:5968.

Logan, A.C., Chow, K.P. George, A. Weinstein, P.D. and J.J. Cebra. 1991. Use of Peyer's patch and lymph node fragment cultures to compare local immune responses to Morganella morganii. Infect. Immun. 59:1024.

Yamamoto, S., Kiyono, H. Yamamoto, M. Imaoka, K. Yamamoto, M. Fujihashi, K. Van Ginkel, F. Noda, M. and Y. A. Takeda. 1997. Nontoxic mutant of cholera toxin elicits Th2-type responses for enhanced mucosal immunity. Proc. Natl. Acad. Sci. 94:5267.

Major, A.S., and C.F. Cuff. 1997. Enhanced mucosal and systemic immune responses to intestinal reovirus infection in beta2-microglobulin-deficient mice. J. Virol. 71:5782.

Letvin, N.L., Kauffman, R.S. and R. Finberg. 1981. T lymphocyte immunity to reovirus: cellular requirements for generation and role in clearance of primary infections. J. Immunol. 127:2334.

London, S.D., Cebra, J.J. and D.H. Rubin. 1989. Intraepithelial lymphocytes contain virusspecific, MHC-restricted cytotoxic cell precursors after gut mucosal immunization with reovirus serotype 1/Lang. Reg. Immunol. 2:98. 
Cuff, C.F., Cebra, C.K. Rubin, D.H. and J.J. Cebra. 1993. Developmental relationship between cytotoxic alpha/beta $\mathrm{T}$ cell receptor-positive intraepithelial lymphocytes and PP lymphocytes. Eur. J. Immunol. 23:1333

Chen, D., Lee, F. Cebra, J.J. and D.H. Rubin. 1997. Predominant T cell receptor Vbeta usage of intraepithelial lymphocytes during the immune response to enteric reovirus infection. J. Virol. $71: 3431$.

Woolverton, C.J., Holt, L.C. Mitchell, D. and R.B. Sartor. 1992. Identification and characterization of rat intestinal lamina propria cells: consequences of microbial colonization. Vet. Immunol. Immunopathol. 334:127.

Lorentz, A., Schwengberg, S. Sellge, G. Manns, M.P. and S.C. Bischoff. 2000. Human intestinal mast cells are capable of producing different cytokine profiles: role of IgE receptor cross-linking and IL-4. J. Immunol. 164:43.

Goodrich, M.E., and McGee, D.W. 1999. Preferential enhancement of B cell IgA secretion by intestinal epithelial cell-derived cytokines and interleukin-2. Immunol. Invest. 28:67.

Bilenker, M., Roberts, A.I. Brolin, R.E. and E.C. Ebert. 1995. Interleukin-7 activates intestinal lymphocytes. Dig. Dis. Sci. 40:1744.

Finkelman, F.D., Madden, K.B. Cheever, A.W. Katona, I.M. Morris, S.C. Gately, M.K. Hubbard, B.R. Gause, W.C. and J.F. Urban. 1994. Effects of Interleukin-12 on immune 
responses and host protection in mice infected with intestinal nematode parasites. J. Exp. Med. 179:1563.

Lai, Y.G., Gelfanov, V. Gelfanova V. Kulik L. Chu, C.L. Jeng, S.W. and N.S. Liao. 1999. IL-15 promotes survival but not effector function differentiation of CD8+ TCRalphabeta+ intestinal intraepithelial lymphocytes. J. Immunol. 163:5943.

Pizarro, T.T., Michie, M.H. Bentz, M. Woraratanadharm, J. Smith, M.F. Foley, E. Moskaluk, C.A. Bickson, S.J. and F. Cominelli. 1999. IL-18, a novel immunoregulatory cytokine, is up regulated in Crohn's disease: expression and localization in intestinal mucosal cells. J. Immunol. 162:6829.

Lillard, J.W., Boyaka, P.N. Hedrick, J.A. Zlotnik, A. and J.R. McGhee. 1999. Lymphotactin acts as an innate mucosal adjuvant. J. Immunol. 152:1959.

Bender, B.S., Tallman, E. Johnson, M.P. and P.A. Small. 1990. Enhancement of anti-influenza cytotoxic T-lymphocyte activity in senescent mice by vaccination in early life. Mech. Ageing Dev. 55:1. 


\section{Addendum to:}

The CTL V $\beta$ Repertoire Induced Following Oral or Systemic Infection with Reovirus 


\section{Introduction}

Additional experiments were performed to determine the effect of the route of infection upon the selection and expansion of virus-specific CTL populations. Some of these experiments were initial pilot studies of our adoptive transfer experiments as described in Chapter 2, wherein we assessed the $\mathrm{V} \beta$ repertoire of $\mathrm{CD} 8^{+}$splenocytes recovered from reovirus-infected CB.17 SCID recipients of purified splenic $\mathrm{T}$ cells from congenic Balb/c donors infected orally or in the hind footpads. We believed adoptively transferred virus-specific cells could be expanded in reovirus infected SCID mice as indirectly evidenced by the clearance of reovirus infection from infected SCID recipients of immune, unfractionated Peyer's patch cells (George et al., 1990). We further expected to be able to see a dramatic proliferative expansion of $\mathrm{CD} 8^{+} \mathrm{T}$ cells, as seen by Zimmermann et al., (1999) following the adoptive transfer of purified T cells from mice previously immunized against vesicular stomatitis virus (VSV) or vaccina virus into naïve mice subsequently infected with VSV of vaccinia. In the second week post-transfer, we found a dramatic expansion of $\mathrm{CD}^{+} \mathrm{T}$ cells in the infected SCID recipient mice consisting of a limited number of $V \beta$ subpopulations that were the same regardless of the route of infection of the donor mice. These data suggest that oral and parenteral reovirus infection of donor mice induce similar responder CTL populations.

\section{Methods}

Mice: Male CB.17 SCID mice were born and housed in microisolator cages within isolation bubbles at the University of Pennsylvania Department of Biology animal care facility. Male Balb/c mice were acquired from Charles River Laboratories (Wilmington, MA) at 8 weeks of age and allowed to acclimate for 1 week before use. Balb/c mice were kept in micro-isolator cages in a pathogen-free room, and all mice were maintained on autoclaved food and water. Virus- 
infected Balb/c mice were housed in a laminar flow hood in a separate room under negative pressure.

Virus: Third passage reovirus T1L stocks were grown in L929 cells. Cells were disrupted by sonication in a buffer of $0.01 \mathrm{M}$ Tris, $0.25 \mathrm{M} \mathrm{NaCl}, 0.01 \mathrm{M} \beta$-mercaptoethanol, and $0.1 \%$ sodium deoxycholate ( $\mathrm{pH} 7.4$ ) and purified by freon extraction followed by step-wise $\mathrm{CsCl}$ gradient centrifugation as described (Smith et al., 1969). Infectious reovirus titers were assessed by plaque assay in L929 cells as described (Cuff et al. 1990).

Animal infections: Orally-infected mice received $3 \times 10^{7}$ plaque-forming units (pfu) of reovirus in $50 \mu \mathrm{l}$ of sterile borate-buffered saline and gelatin by gavage using a stainless steel feeding tube attached to a $1 \mathrm{ml}$ syringe. Hind footpad infected mice received $1.5 \times 10^{7} \mathrm{pfu}$ of reovirus in $50 \mu \mathrm{l}$ of sterile borate-buffered saline and gelatin in each hind footpad. SCID recipient mice received $3 \times 10^{7}$ pfu of reovirus in $100 \mu$ l total of sterile borate-buffered saline and gelatin by intraperitoneal injection.

Preparation of spleen cells: Single cell suspensions were prepared by expressing spleens through sterile nylon mesh. Splenocytes were depleted of red blood cells by incubation in a hypotonic solution of $\mathrm{NH}_{4} \mathrm{Cl}$ and Tris-HCl. Splenocytes were washed 3 times in medium consisting of RPMI-1640 (Biowhittaker, Walkersville, MD) supplemented with 10\% FBS (Hyclone, Logan, VT), 2mM L-glutamine (Biowhittaker), 100U penicillin/ml, 0.1 $\mu \mathrm{g}$ streptomycin/ml (Biowhittaker), and 50 $\mu \mathrm{M} \beta$-mercaptoethanol, (TCM). Cell viability was determined by trypan blue exclusion.

Adoptive transfer: RBC-depleted donor lymphocytes collected from mice that were infected 30 days previously were depleted of surface Ig-positive B cells by three successive incubations on sterile $16 \times 100 \mathrm{~mm}$ polystyrene bacteriology grade petri dishes (Falcon) coated 
with $100 \mu \mathrm{g} / \mathrm{ml}$ goat anti-mouse $\operatorname{IgG}, \operatorname{IgM}$ and IgA antibody (Rockland) in a $0.15 \mathrm{M}$ Tris buffer (pH 9) for 45 minutes at $4^{\circ} \mathrm{C} .1 .5 \times 10^{7}$ non-adherent $\mathrm{T}$ cells were resuspended in $1 \mathrm{ml}$ of sterile PBS, and were injected intraperitoneally into reovirus-infected SCID recipient mice using a 20gauge needle.

Flow cytometric analysis of effector cells: RBC-depleted mononuclear splenocytes were stained with phycoerythrin (PE)-conjugated anti-CD8 $\alpha$ mAb (CALTAG, Burlingame, CA), and fluorescein isothiocyanate (FITC)-conjugated mAbs to either CD4 or V $\beta$ 2, 4, 5, 6, 7, $8.1 / 8.2,8.3,9,10,11,12,13,14,17$, (Pharmingen). Some cells were also stained with a biotinylated anti-V $\beta 3$ mAb (Pharmingen) followed by FITC-avidin (CALTAG). Percentages of cells staining positive for each marker were determined by analysis on a FACScan flow cytometer (Becton-Dickinson, Franklin Lakes, NJ), and results were further analyzed using WinMIDI 2.8 software (Joseph Trotter, Scripps Research Institute, La Jolla, CA).

Statistical analysis: Means and standard deviations were calculated for each V $\beta$ population expressed among all groups of mice and tissues assessed by flow cytometry. Statistically significant differences in $\mathrm{V} \beta$ expression were determined by one - way analysis of variance (ANOVA) followed by the Tukey test using Sigmastat 2.0 software (Jandel Scientific, Chicago, IL).

\section{Results and Discussion}

Adoptively transferred, reovirus-primed $\mathrm{CD} 8^{+}$cells from immunocompetent donors expand in reovirus-infected SCID recipients. Splenocytes from Balb/c mice infected orally or in the hind footpads with $3 \times 10^{7}$ pfu of reovirus 30 days previously were depleted of B cells by panning on anti-murine Ig coated petri plates. $2.0 \times 10^{7}$ pooled enriched T cells were transferred into isogenic SCID CB.17 recipients ( $n=12$ recipients of per orally infected donors; $n=10$ 
recipients of footpad-infected donors) themselves infected with $3 \times 10^{7}$ pfu of reovirus 4 days previously. Splenocytes from the recipient mice were isolated at day 9, 11, and 13 post-transfer, and assessed for $\mathrm{CD}^{+}$and $\mathrm{CD} 8^{+} \mathrm{T}$ cell number and TCR V $\beta$ repertoire by flow cytometry. A progressive increase in the number of $\mathrm{CD} 4^{+} \mathrm{T}$ cells recovered from the spleens of infected recipient mice is seen in the second week post-transfer, with $8.3 \times 10^{6} \pm 2.5 \times 10^{6}, 17.6 \times 10^{6} \pm$ $11.0 \times 10^{6}$, and $9.4 \times 10^{6} \pm 0.7 \times 10^{6} \mathrm{CD}^{+}$cells, and $8.1 \times 10^{6} \pm 7.6 \times 10^{6}, 15.8 \times 10^{6} \pm 1.8 \times 10^{6}$, and $20.8 \times 10^{6} \pm 9.4 \times 10^{6}, \mathrm{CD}^{+}$cells recovered on days 9,11 , and 13 , from SCID recipients of cells from orally infected and footpad infected donors, respectively (Figure 28A and B, respectively). A more dramatic increase is seen among recovered $\mathrm{CD} 8^{+} \mathrm{T}$ cells. $9.8 \times 10^{6} \pm 5.8 \times 10^{6}, 30.5 \times 10^{6} \pm$ 19.9 $910^{6}$, and $25.4 \times 10^{6} \pm 9.0 \times 10^{6} \mathrm{CD} 8^{+}$cells, and $5.8 \times 10^{6} \pm 4.3 \times 10^{6}, 32.9 \times 10^{6} \pm 11.0 \times 10^{6}$, and $30.9 \times 10^{6} \pm 9.8 \times 10^{6} \mathrm{CD}^{+}$cells were recovered on days 9,11 , and 13 from SCID recipients of cells from orally infected and footpad infected donors, respectively (Figure 28A and B). Recovered $\mathrm{CD} 8^{+}$cells outnumber $\mathrm{CD} 4^{+}$cells by approximately $2: 1$ after day 9 post-transfer in recipient mice of both orally and footpad infected donors.

A dramatic and progressive increase in the percentage of $\mathrm{V} B 3^{+} \mathrm{CD} 8^{+}$cells was observed with increasing time post-transfer for SCID recipients of both orally infected and footpad infected donors (percentage data not shown). The absolute numbers of $\mathrm{CD} 8^{+}$splenocytes of the assessed $\mathrm{V} \beta$ repertoire were determined for both groups at all time-points (Figure 29). Again, a progressive increase with time in the number of $\mathrm{V} \beta 3^{+}$cells was seen in both groups of recipient mice. One-way ANOVA showed that among SCID recipients of footpad infected mice there is a significant increase in the number of ${\mathrm{V} \beta 3^{+}}^{+}$cells from day 9 to day 11 and day 13 post-transfer as determined by the Tukey test $(p<0.05)$. Similar changes are seen in the recipients of cells from footpad-infected donors. Other minor changes were observed for some $V \beta$ subpopulations, 
notably the $\mathrm{V} \beta 8.3^{+}$and $\mathrm{V} \beta 9^{+} \mathrm{CD} 8^{+}$subpopulations, but none of these were as striking as the change in the $\mathrm{V} B 3^{+} \mathrm{CD} 8^{+}$cells, nor were any other changes found to be statistically significant by one way ANOVA.

Our data indicate that the $\mathrm{V} B 3^{+} \mathrm{CD} 8^{+} \mathrm{T}$ cell subpopulation was primed in vivo following either oral or parenteral reovirus infection of donor mice, as this population was greatly expanded in the infected CB.17 SCID recipient mice. Furthermore, no significant differences were found among any of the TCR V $\beta$ subpopulations of $\mathrm{CD} 8^{+} \mathrm{T}$ cells recovered from the recipients of cells from orally infected or parenterally infected donors. Our data suggest the finding that the route of infection does not select distinct responder CTL populations, at least at the level of the TCR V $\beta$ repertoire, is true for the murine $\mathrm{Balb} / \mathrm{c}$ strain as well as the $\mathrm{C} 3 \mathrm{HeB} / \mathrm{FeJ}$ strain. 


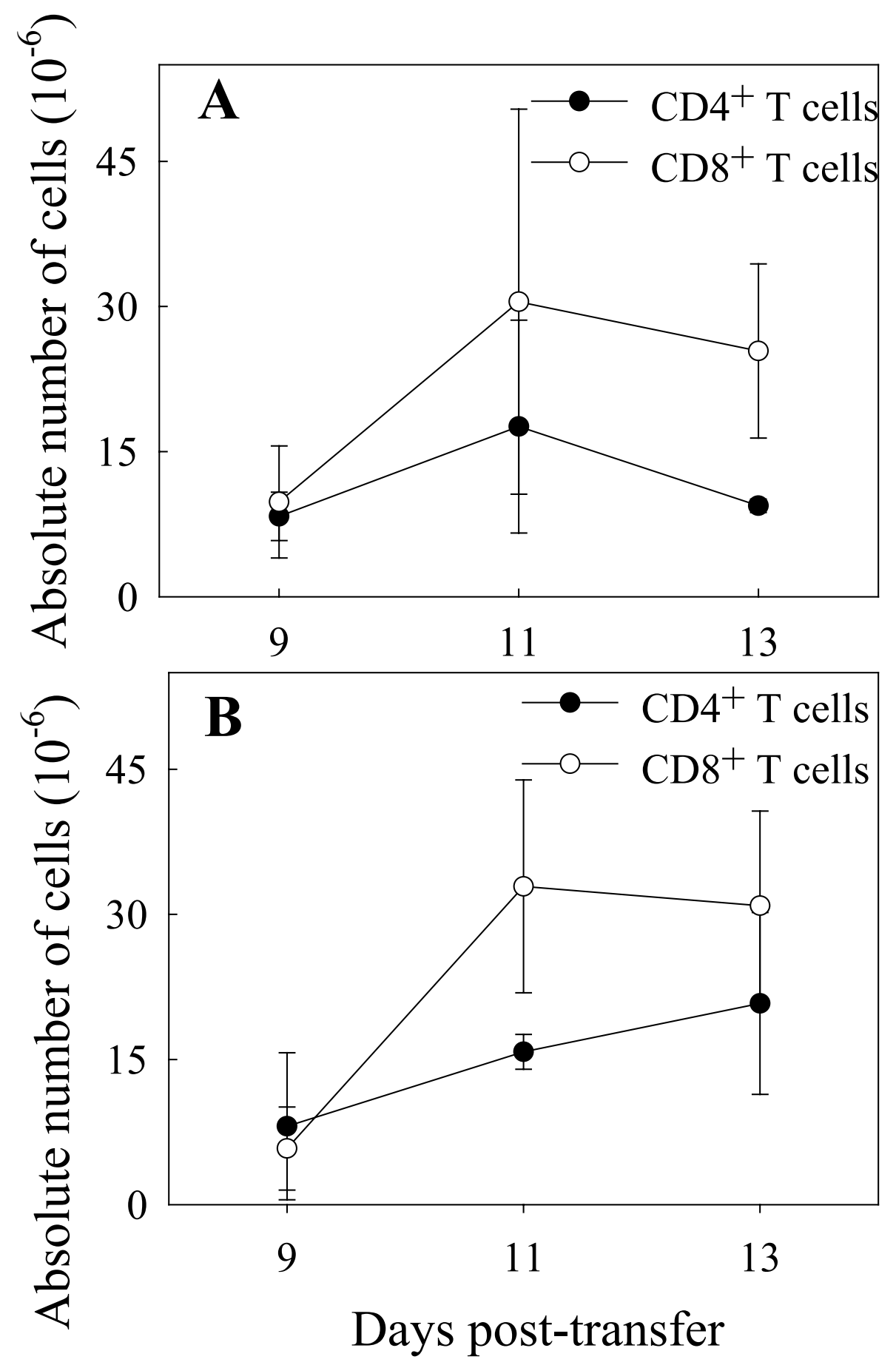


Figure 28. CD4+ and CD8+ cell numbers recovered from reovirus infected SCID recipient mice of immune donor T cells. Infected CB.17 SCID mice received $1.5 \square 107$ purified splenic T cells from orally infected (A) and hind footpad infected (B) donor mice as described in methods. Splenocytes were isolated from recipient SCID mice at days 9,11 , or 13 posttransfer and assessed for $\mathrm{CD}^{+}(\bullet)$ and $\mathrm{CD8}^{+}(\circ) \mathrm{T}$ cells by flow cytometry. Data are given as (mean \pm standard deviation $)\left(\times 10^{-6}\right.$ cells $)$. 


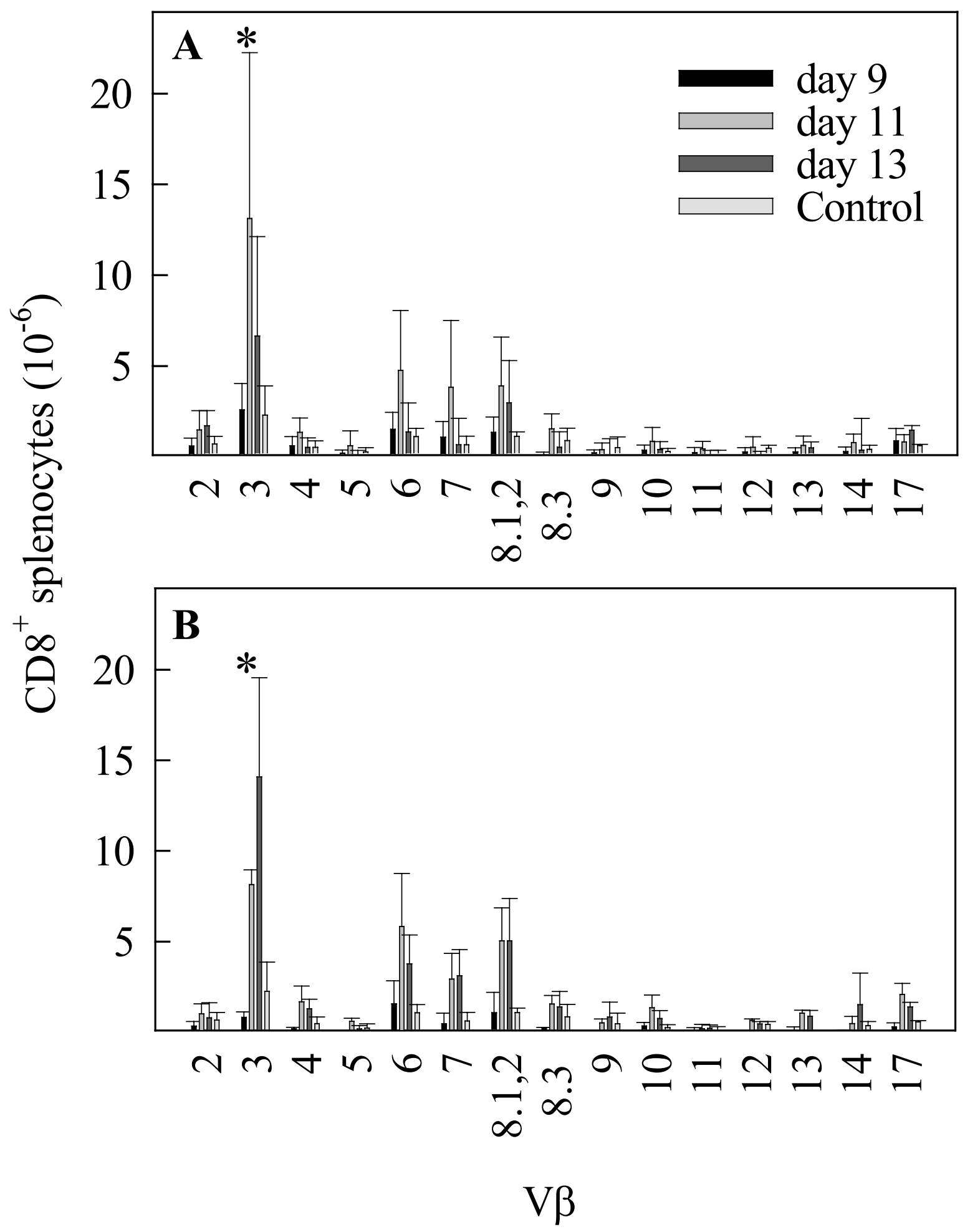


Figure 29. V $\beta$ repertoire, in absolute numbers, of $\mathrm{CD8}+\mathrm{T}$ cells recovered from reovirusinfected SCID recipients of purified T cells from immune donor mice. Infected CB.17 SCID mice received $1.5 \times 107$ purified splenic $T$ cells from orally infected (A) and hind footpad infected (B) donor mice as described in methods. Splenocytes were isolated from recipient SCID mice at days 9,11 , or 13 post-transfer and assessed for changes in the $V \beta$ repertoire $\mathrm{CD8}^{+} \mathrm{T}$ cells by flow cytometry, and absolute numbers were calculated.

Data are given as (mean \pm standard deviation $)\left(\times 10^{-6} \mathrm{CD} 8^{+}\right.$cells $)$.

* significantly different by one way ANOVA followed by the Tukey test, $(p<0.05)$. 


\section{Acknowledgements}

We wish to thank Dr. John Cebra for the donation of his CB.17 SCID mice and facilities to our work. We also wish to thank Alec MacKay for his assistance with flow cytometry. We further wish to thank the staff of the University of Pennsylvania animal care facility for maintaining the SCID mice. 


\section{References}

Cuff, C.F., Lavi, E., Cebra, C.K., Cebra, J.J., and D.H. Rubin. 1990. Passive immunity to fatal reovirus serotype 3 -induced meningoencephalitis mediated by both secretory and transplacental factors in neonatal mice. J. Virol. 64:1256-1263.

George, A., Kost, S.I., Witzleben, C.L., Cebra, J.J., and D.H. Rubin. 1990. Reovirus-induced liver disease in severe combined immunodeficient (scid) mice. A model for the study of viral infection, pathogenesis, and clearance. J. Exp. Med. 171:929-934.

Smith, R.E., Zweerink, H.J., Joklik, W.K. 1969. Polypeptide components of virions, top component and cores and reovirus type 3. Virology. 39:791-810.

Zimmermann, C., and H. Pircher. 1999. A novel approach to visualize polyclonal virus-specific $\mathrm{CD} 8^{+} \mathrm{T}$ cells in vivo. J. Immunol. 162:5178-5182. 


\title{
Human Intestinal Immunity
}

\author{
Jonathan R. Fulton, Cynthia A. Cunningham, \\ and Christopher F. Cuff \\ Department of Microbiology and Immunology, \\ West Virginia University \\ School of Medicine
}

In press in Colonic Diseases. Timothy Coke M.D. Editor

Humana Press Inc., Louiseville, KY. 


\section{Introduction}

The gastrointestinal system is essentially a long muscular tube, the functional surface of which is a thin, mucus-coated layer approximately $1 \mathrm{~mm}$ thick, that is joined at both ends with the external integument and thus is a contact surface with the external environment (MacDonald $e t$ al., 1999). The mucosal surface area of the adult human intestine is estimated to be approximately $300 \mathrm{~m}^{2}$ (Brandtzaeg et al., 1989). This surface is constantly exposed to antigens, which proximally is mostly of dietary origin, and distally tends to be bacterial products derived from colonic flora. Providing a protective barrier at this external surface is complicated by the need to selectively absorb nutrients. To prevent the colonization and/or invasion of the intestinal mucosal by foreign organisms, the intestine makes use of a number of innate and adaptive defense factors. This chapter provides a broad overview of immune responses in the intestine.

\section{Innate immunity}

A palisade of columnar intestinal epithelial cells (IEC) with interspersed mucoussecreting goblet cells maintains the first line of innate mucosal defense. Mucous from the goblet cells sheaths the mucosal epithelium, and together with the glycoproteins of the IEC glycocalyx forms a size-restrictive permeability barrier against lumenal antigens (Pitman and Blumberg, 2000). Tight junctions between adjacent IEC serve to prevent intercellular passage of antigens and organisms into the intestinal tissues. Thus, under ideal conditions, the majority of lumenal contents that gain access to the intestinal tissues are small nutrient molecules transported transcellularly across the IEC.

The integrity of the IEC barrier in the villi and crypts is maintained by constant proliferation of epithelial stem cells located roughly halfway down the villi, the progeny of which migrate either downward into the crypt or upward to the tips of the villi. Progeny cells 
initially have the ability to differentiate into a number of epithelial cell types, such as absorptive IEC, Paneth cells, and goblet cells, undergoing progressive differentiation during migration. These cell types have specialized functions in protection of the mucosa. For instance, Paneth cells, which are located at the bases of the crypts, produce a number of exocrine antimicrobial factors, such as the anion-binding, pore-forming $\alpha$-defensins (cryptins) (Jones and Bevin, 1992), peptidoglycan hydrolyzing lysozyme (Valnes et al., 1984), and phospholipase A2 (Kiyohara et al., 1992), that contest microbial colonization of the crypt epithelium. Goblet cells, in addition to secreting the mucus barrier (Jabbal et al., 1976), also produce trefoil proteins (Podolsky et al., 1993), factors that enhance IEC migration toward sites of injury and are thus believed to play a role in maintaining the integrity of the epithelial barrier (Modlin and Poulsom, 1997).

\section{Adaptive Immunity}

\section{Organization and Location of Intestinal Lymphocytes:}

While passive and active innate factors of the intestinal epithelium provide a basic outermost layer of defense against a broad assortment of environmental antigens and organisms, a vast array of cells of hematopoietic origin are dispersed throughout the underlying lamina propria, clustered in highly organized secondary lymphoid tissues, and intercalated within the IEC palisade. These bone marrow derived cells include $\mathrm{CD} 4^{+} \mathrm{T}$ helper cells (Th), which support and direct many of the effector functions of other cells, $\mathrm{CD} 8^{+} \mathrm{T}$ cells and Natural Killer (NK) cells, which mediate cytotoxicity against infected, transformed, or stressed self cells. Other cells involved include B lymphocytes, which produce antigen specific immunoglobulin of primarily the IgA isotype and lesser quantities of $\operatorname{IgM}$ and $\operatorname{IgG}$ (Brantzaeg et al., 1989), macrophages, dendritic cells, and tissue granulocytes such as mast cells and eosinophils. 
Lymphoid tissues of the intestine are typically categorized into 2 types: 1 ) inductive tissues, wherein naïve $\mathrm{T}$ and $\mathrm{B}$ cells as well as antigen-experienced memory cells are primed by gut lumenal antigens and then induced to proliferate, and 2) effector tissues, the sites to which primed lymphocytes migrate and mediate protective immune function (Mowat and Viney, 1997). Inductive tissues include the Peyer's patches (PP), the mesenteric lymph nodes (MLN), the appendix, isolated lymphoid follicles (LF), and potentially such structures as the lymphocytefilled villi (Moghaddami et al., 1999). Effector tissues include the intestinal lamina propria underlying the villus and crypt absorptive epithelium, and the T lymphocyte-dominated intraepithelial lymphocyte (IEL) compartment (MacDonald et al., 1999). Collectively, all these tissues are referred to as the gut-associated lymphoid tissue (GALT). Inductive lymphoid follicles, which are aggregates of $\mathrm{B}$ and $\mathrm{T}$ cells, interdigitating dendritic cells, and macrophages separated from the lumenal contents by specialized follicle associated epithelium (FAE), are distributed along the length of the intestine from the duodenum to the ano-rectal junction (Moghaddami et al., 1999). While generally located in the lamina propria of the antimesenteric gut wall, human follicles are distributed randomly around the circumference of the gut. While generally not macroscopically visible, on histological examine they can be extensive, and may be either entirely contained within the lamina propria, or may have long extensions under the muscularis mucosa, creating structures previously identified as sub-mucosal lymphoid aggregates (Moghaddami et al., 1999).

The largest aggregates of inductive lymphoid tissue in the GALT, the PP are considered the premier priming tissue of intestinal immunity (Kelsall et al., 1996). PP, defined as collections of 5 or more lymphoid follicles, are roughly analogous to peripheral lymph nodes, and are grossly located toward the antimesenteric wall of the intestine. Histologically and 
functionally, the lymphoid follicle and appendix are very similar to the PP, although some question still remains as to whether the appendix may also serve as a primary B cell organ in the human perinatal gut (Dasso et al., 2000). The quantity of follicular lymphoid tissue in the gut is considerable; there are estimated to be 11,000 to 14,000 scattered LF in the large intestine alone (Gebbers et al., 1992).

The lumenal face of the PP, as with all gut follicular tissues, is covered with a specialized dome of epithelial cells, known as follicle associated epithelium (FAE) (Jacob et al., 1987). Among the conventional columnar epithelial cells of the FAE are interspersed thin, flat microfold (M) cells that lack the long, organized apical microvilli, glycocalyx, and mucus coating, that characterize the villus absorptive epithelium. The lack of these covering structures, which form a size-selective permeability barrier to prevent access of large antigenic molecules and organisms from the absorptive epithelium, make $\mathrm{M}$ cells natural portals of ingress for lumenal antigens and organisms into the Peyer's patches (Neutra et al., 1996). Indeed, in animal studies intestinal pathogens such as Salmonella typhi (Kohbata et al., 1986), Shigella flexneri (Wassef et al., 1989), Yersinia enterocolitica (Grutzkau et al. 1990), reovirus (Wolfe et al., 1983), poliovirus type 1 (Sicinski et al., 1990), and Cryptosporidium (Marcial and Madara, 1986) are believed to bind to glycoproteins on the $M$ cell surface, facilitating their ingress. $M$ cells also have been shown to express Fcalpha receptors (FcoR) that selectively allow the binding and uptake of lumenal antigens bound by secreted IgA, the immunoglobulin most associated with mucosal humoral immunity (Neutra, 1999). Additionally, particulate and soluble antigen may be non-specifically internalized and transcytosed by M cells.

Although M cells have been demonstrated to contain lysosomal vesicles, express major histocompatibility class I and, in the small but not the large intestine, class II molecules (Bjerke 
and Brandtzaeg, 1988; Ueki et al., 1995), and are in intimate contact with a population of T and B lymphocytes actually encased within folds of the M cell (Farstad et al., 1994), it remains uncertain whether they play a role as antigen presenting cells. However, $\mathrm{M}$ cells transcellularly translocate lumenal antigens and organisms into the sub-epithelial dome region of the Peyer's patch, which immediately underlies the FAE (Mowat and Viney, 1997). This area is rich in macrophages and immature dendritic cells that can internalize, process, and present peptide antigen epitopes in association with MHC class I and class II molecules to antigen-specific T cells (Mowat and Viney, 1997). Maturing antigen-bearing dendritic cells and macrophages subsequently migrate from the sub-epithelial dome region deeper into the $\mathrm{T}$ cell enriched parafollicular regions, where they join with networks of interdigitated dendritic cells that serve to prime $\mathrm{CD} 4^{+}$Th cells and cytolytic $\mathrm{CD} 8^{+} \mathrm{T}$ cells (Steinman et al., 1997).

\section{Cellular Immunity}

T-helper cells:

Adaptive immune responses can be broadly divided into two arms: 1) the cellular response, mediated primarily $\mathrm{CD} 4^{+}$and $\mathrm{CD} 8^{+} \mathrm{T}$ cells, and the myeloid cells they influence, and 2) the humoral response, consisting of B cells and the specific antibodies they produce under the influence primarily of $\mathrm{CD} 4^{+}$Th cells. Central to both arms of adaptive immunity is the priming of antigen-specific naive $\mathrm{T}$ lymphocytes by antigen-presenting dendritic cells. Interaction of $\mathrm{T}$ cells bearing T cell receptors (TCR) specific for antigenic epitopes expressed in association with MHC class I or II molecules on the surface of dendritic cells results in mutual stimulation and activation of the $\mathrm{T}$ cell and dendritic cell. Binding of dendritic cell CD40 to T cell CD40 ligand (CD40L) induces the dendritic cells to upregulate expression of $\mathrm{T}$ cell co-stimulatory molecules B7.1 and B7.2, which in turn interact with T cell surface molecule CD28 to augment the 
stimulatory signaling of the TCR-CD3 complex with its epitope (McLellan et al., 1996). These multiple interactions required to initiate a T-cell mediated immune response are important controls in preventing the development of aberrant immune responses.

$\mathrm{CD}^{+} \mathrm{T}$ cells recognizing antigen through the TCR and receiving co-stimulation through B7-CD28 ligation, up regulate both T cell growth factor IL-2 production (Petro et al., 1995), and expression of the high affinity IL-2 receptor alpha subunit (TAC; CD25) (Kremer et al., 1998), allowing for autocrine induction of proliferation. Activated progeny $\mathrm{CD} 4^{+} \mathrm{T}$ cells may then either differentiate into effector cells, or migrate from the PP via efferent microlymphatic vessels to complete differentiation and mediate effector function in distant tissues (Dunkley and Husband, 1987).

Effector $\mathrm{CD}^{+} \mathrm{T}$ cells are classified as either T-helper type 1 (Th1) or T-helper type 2 (Th2), and are defined by the cytokines that they produce. Th1 cells produce IL-2, IFN- $\gamma$, and TNF- $\alpha$. IFN- $\gamma$ activates macrophages, up regulates MHC class II expression of APC (Farrar and Schreiber, 1993), induces activated B cells to undergo immunoglobulin heavy chain isotype switching to $\mathrm{IgG} 2$, and inhibits the establishment of Th2 responses (Farrar and Schreiber, 1993). Th1 cytokines, in particular IFN- $\gamma$ and IL-2, can also augment $\mathrm{CD} 8^{+}$cytotoxic $\mathrm{T}$ cell responses (Biron, 1994). Th2 effector T cells produce predominately IL-4, IL-5, IL-6, and IL-10 (McHugh et al., 1996). These cytokines act on humoral responses; IL-4 induces isotype class switching to IgE and IgG1 (Rousset et al., 1991), whereas IL-5, IL-6, and IL-10 supports proliferation of activated B cells, and their differentiation into antibody-secreting plasma cells (Burdin et al., 1995). Selection of Th1 versus Th2 responses is thought to be influenced by the local cytokine milieu (Shibuya et al., 1998), concurrent immune responses (McHugh et al., 1995), the nature of the antigen (McHugh et al., 1996), and the APC (Schmitz et al., 1993), and individual genetic 
predisposition (Shibuya et al., 1998). It is thought that T-helper cells are the most important lymphocyte population in immunopathological inflammatory bowel diseases.

Cytotoxic T lymphocyte responses:

$\mathrm{CD} 8^{+} \mathrm{T}$ cells are responsible for killing cells infected with intracellular pathogens by effector mechanisms such as membrane pore-forming perforin and granzyme mediated cytotoxicity, as well as by inducing apoptosis of target cell by Fas-FasL interactions. CD8 ${ }^{+}$cells can also kill intracellular bacteria such as mycobacteria by means of the antimicrobial protein granulysin. Naïve $\mathrm{CD} 8^{+} \mathrm{T}$ cells, are activated by antigen expressed in association with $\mathrm{MHC}$ class I molecules by dendritic cells in the parafollicular $\mathrm{T}$ cell area of the PP. Effector $\mathrm{CD} 8^{+} \mathrm{T}$ cells produce cytokines such as IFN- $\gamma$, IL-4, and small amounts of IL-2, which can influence other aspects of immune system. In addition, evidence exists in animal and human models that $\mathrm{CD} 8^{+} \mathrm{T}$ cells can suppress immune responses.

\section{Effector $\mathbf{T}$ cells in the Intestine}

\section{Overview:}

T lymphocytes in the intestinal lamina propria and intraepithelial lymphocyte compartment are numerous, and are phenotypically, functionally, and developmentally diverse. In general terms, LP and some IEL $\mathrm{T}$ cells can be considered as conventional antigen experienced, chronically activated immigrant lymphocytes from GALT and peripheral immune compartments. Four general functions can be ascribed to both LP and IEL T cells: Th cell support of secretory IgA and IgM mediated immune exclusion; protection of the mucosa against invasive organisms; regulation and attenuation of inflammatory responses; repair and maintenance of the integrity of the epithelial barrier. These functions are mediated by secreted cytokines, and direct cell contact dependent ligation of signal-transducing cell surface molecules. 
As cytokine profiles, cell surface receptor expression, and activation requirements of mucosal effector $\mathrm{T}$ cells are often quite different, it is likely that the above mentioned functions are mediated, with varying amounts of overlap, by distinct $\mathrm{T}$ cell subsets.

\section{Lamina propria $\mathrm{T}$ cells:}

Lamina propria $\mathrm{T}$ lymphocytes are $55 \% \mathrm{CD} 4^{+}, 24 \% \mathrm{CD} 8^{+}$, similar to percentages in the peripheral blood (Schieferdecker et al., 1992). Most $\mathrm{CD}^{+} \mathrm{T}$ cells (95\%) use the $\alpha \beta$ form of the TCR (Ullrich et al., 1990), again similar to the peripheral blood, and although LP $\gamma \delta \mathrm{TCR}^{+}$cells account for $<5 \%$ of $\mathrm{T}$ cells, they are slightly enriched in the LP as compared to the systemic periphery. The LP also contains a minor T cell population that expresses both CD4 and CD8 (14\%) of unknown function (Abuzakouk et al., 1998). These so-called 'double positive' cells are rarely found in the peripheral blood. LP T cells bear surface marker phenotypes associated with previous antigen-experience such as CD45RO (96\%), cellular adhesion molecules CD2 (Schieferdecker et al., 1992), $\alpha_{4} \beta_{7}$ (70\%) (Farstad et al., 1996), $\alpha_{E} \beta_{7}(52 \%)$, and basement membrane binding CD44 (>80\%) (Ebert and Roberts, 1996). LP T cells proliferate at relatively low levels, and are refractory to TCR-specific antigen mediated proliferative responses, although proliferation can be induced by ligation of alternative $\mathrm{T}$ cell stimulation molecules such as $\mathrm{CD} 2$ and CD28 (De Maria et. al., 1993), and epithelial goblet cell derived cytokine IL-7 (Watanabe et al., 1995). Nevertheless, many LP T cells are constitutively activated as evidenced by the expression among roughly $15 \%$ of $\mathrm{CD} 4^{+}$and $\mathrm{CD} 8^{+}$cells of $\mathrm{CD} 25$, the IL-2R alpha subunit referred to as TAC (Zeitz et al., 1988) and the apoptosis-inducing FasL (De Maria et al., 1996). Furthermore, LP T cells were shown to spontaneously produce IFN- $\gamma$ and IL-4 ex vivo. Additionally, high levels of both Th1 cytokines such as IFN- $\gamma$ (Gonsky et al., 2000) IL-2

(Gonsky et al., 1998), and TNF- $\alpha$ (Targan et al., 1995) and Th2 cytokines such as IL-4 (Targan 
et al., 1995), IL-5 (Boirivant et al., 1996), and IL-10 (Braunstein et al., 1997) can be induced by ligation of CD2. Together the available data suggests that these cells are antigen-experienced but somewhat refractory to re-activation. This physiologic state of T-cells in normal LP probably also contributes to the state of immunologic tolerance of the intestinal immune system. Perhaps the most important function of LP T cells is the support of B cell proliferation and differentiation. Differentiation of antigen-experienced B cells into pIgA secreting plasma cells is promoted by T cell cytokines TGF- $\beta$, IL-5, and IL-10, whereas IL-6 supports IgA, IgM, and IgG3 production (Riordan et al., 1998). Compelling evidence exists that LP T cells are constitutively activated to support B cell function, as LP T cells co-cultured with LPS-stimulated LP B cells provided Th cell support for secretion of IgA and IgM, whereas autologous PB T cells did not support IgA and IgM production (Danis and Heatley, 1987). Interestingly, TGF- $\beta$, IL-6 (Goodrich and McGee, 1998), and IL-15 (Hiroi et al., 2000) produced by IEC are thought to augment LP pIgA production in vivo by B2 cells and B1 cells, respectively, suggesting a complex array of factors collectively involved in mucosal immunoregulation.

\section{Intraepithelial lymphocytes:}

In terms of phenotype, effector mechanisms, priming, and ontogeny, LP T cells are similar to other peripheral antigen-experienced $\mathrm{T}$ cells, yet contrast dramatically with IEL. IEL are a dispersed effector lymphocyte population intercalated within the IEC palisade just above the IEC basement membrane, and thus are in intimate contact with the columnar epithelium. The IEL compartment consists of $\mathrm{T}$ lymphocyte-like cells, with diverse and often unconventional phenotypes and developmental backgrounds. Most IEL bear the $\mathrm{CD}_{4} 5 \mathrm{RO}^{+}$phenotype of antigenexperienced cells, and the integrin $\alpha_{E} \beta_{7}$ (Jarry et al., 1990), the ligand for which is E-cadherin expressed on IEC (Higgins et al., 1998). IEL also express HLA-DR and decreased levels of 
TCR/CD3 as compared to PBL, possibly indicating recent activation (Abuzakouk et al., 1996). Up to $90 \%$ of IEL bear the CD8 phenotype, most of which the conventional heterodimeric form CD8 $\alpha \beta$. About $10 \%$ of IEL express CD4, some of which also co-express CD8 in the unconventional homodimeric $\mathrm{CD} 8 \alpha \alpha$ form. $\mathrm{CD} 4{ }^{+}$IEL are found throughout the length of the intestine, and may respond to antigen presented on HLA-DR by IEC (Hoang et al., 1992). Additionally, small populations of $\mathrm{CD}^{-} \mathrm{CD}^{-}$cells are found in the IEL compartment predominantly in the large intestine. Most IEL are $\alpha \beta \mathrm{TCR}^{+}$, with significant fraction of $\gamma \delta \mathrm{TCR}^{+}$ cells that variably increases in quantity from $2 \%$ to $10 \%$ in the duodenum up to approximately $40 \%$ in the large intestine (Trejdosiewicz et al., 1989; Ullrich et al., 1990). While $\alpha \beta \mathrm{TCR}^{+} \mathrm{IEL}$ can express any of the CD8 and CD4 phenotypes, the most prevalent population is the seemingly conventional CD8 $\alpha \beta^{+} \alpha \beta \mathrm{TCR}^{+}$IEL. Of $\gamma \delta \mathrm{TCR}^{+}$IEL, about $58 \%$ express the homodimeric $\mathrm{CD} 8 \alpha \alpha$, with the remainder being $\mathrm{CD}^{-} \mathrm{CD}^{-}$(Deusch et al., 1991).

The distinct phenotypes may correlate with ontogeny. Numerous studies undertaken in animals have suggested that $\mathrm{CD} 8 \alpha \alpha^{+} \alpha \beta \mathrm{TCR}^{+}$and $\gamma \delta \mathrm{TCR}^{+}$IEL may be derived from extrathymic maturation pathways. This is less clear in humans. Nevertheless, these distinct phenotypes do correlate with function and specificity. It is well established that IEL with the conventional $\mathrm{CD} 8 \alpha \beta^{+} \alpha \beta \mathrm{TCR}^{+}$phenotype contain conventional antigen-specific cytotoxic $\mathrm{T}$ cell populations (Chardes et al., 1994; Chen et al., 1997), and that these CTL are derived from CD8 ${ }^{+}$ T cells primed in Peyer's patches (Cuff et al., 1993) and other secondary lymphoid tissues (Sydora et al., 1996), and assume effector function upon TCR mediated recognition of antigen. Analysis of the TCR $\beta$ chain sequences have demonstrated that while CD8 $\alpha \beta^{+}$and $\operatorname{CD} 8 \alpha \alpha^{+} \mathrm{T}$ cells are not derived from the same precursor $\mathrm{T}$ cells, they are both the clonal progeny of very restricted group of progenitors as compared to peripheral blood $\mathrm{CD} 8^{+} \mathrm{T}$ cells (Pluschke et al., 
1994). This restricted clonality is thought to derive from a chronic expansion and of IEL of certain antigenic specificies by enteric microfloral or dietary antigens. However, $\operatorname{CD} 8 \alpha \beta^{+}$ $\alpha \beta$ TCR $^{+}$IEL bearing identical TCR $\beta$ chain sequences may be simultaneously broadly distributed throughout the length of the intestine and locally enriched (Regnault et al., 1995), and also found within the thoracic duct and LP (Arstila et al., 2000), indicating that clonal populations of IEL may proliferate locally, and that progeny cells may migrate out of the epithelium and recirculate to distant sections of the intestine (Arstila et al., 2000).

While $\mathrm{CD} 8 \alpha \beta^{+} \alpha \beta \mathrm{TCR}^{+}$IEL are responsive to TCR mediated recognition of antigen, $\mathrm{CD} 8 \alpha \alpha^{+}$ $\alpha \beta \mathrm{TCR}^{+}$and $\gamma \delta \mathrm{TCR}^{+}$IEL are much less responsive to TCR mediated activation (Barrett et al., 1992). However, IEL are stimulated to proliferate and assume effector function by alternative pathways such as ligation of IEL CD2 (Van Houten et al., 1993) and thus may be activated in vivo by contact with the IEC CD2 ligand LFA-1 (Kvale et al., 1992). Additionally, IEC express surface glycoproteins 180 , which can stimulate IEL proliferation through direct binding of CD8 (Li et al., 1995). Furthermore, IEL proliferation can be induced by goblet cell secreted IL-7, which is augmented by LP CD4 ${ }^{+}$T cell TNF- $\alpha$ and IL-2 (Ebert, 1998), and by direct contact with LP myofibroblasts underlying the IEC basement membrane (Roberts et al., 1997). CD8 $\alpha \beta^{+}$ $\alpha \beta \mathrm{TCR}^{+} \mathrm{IEL}$ are MHC class I restricted, mediate effector function by perforin and FasL mediated cytotoxicity (Corazza et al., 2000), and produce cytokines such as IFN- $\gamma$ (Fan et al., 1998), IL-8, and TNF- $\alpha$ (Lundqvist et al., 1996), and chemokines such as MIP-1 $\beta$ (Mattapallil et al., 1998). CD8 $\alpha \alpha^{+} \alpha \beta \mathrm{TCR}^{+}$IEL and $\gamma \delta \mathrm{TCR}^{+}$IEL also have the ability to mediate cytotoxicity and secrete IFN- $\gamma$, although both subsets have been shown to recognize alternative non-classical MHC molecules on IEC, such as CD1d (Panja et al., 1993; Sydora et al., 1996). Indeed, $\gamma \delta$ TCR ${ }^{+}$ IEL expressing the TCR V $\delta 1$ chain, a unique, defining phenotype of the majority of IEL $\gamma \delta \mathrm{TCR}^{+}$ 
IEL (Deusch et al., 1991), respond to IEC expression of MHC Class I like (MICA) molecules MICA-A and MICA-B, polymorphic molecules upregulated on stressed or damaged epithelium (Groh et al., 1998; Griffith et al., 1998). Additionally, some murine $\alpha \beta \mathrm{TCR}^{+}$and $\gamma \delta \mathrm{TCR}^{+} \mathrm{IEL}$ have been shown to proliferate and secrete IL-3, myeloid cell growth factor GM-CSF, and IFN- $\gamma$ in response to soluble 65-kDa mycobacterial heat shock protein (HSP) (Beagley et al., 1993), which has been thought to be a cross-reactive antigen with mammalian HSP. In murine studies, $\mathrm{CD} 8 \alpha \alpha^{+} \alpha \beta \mathrm{TCR}^{+}$IEL and $\gamma \delta \mathrm{TCR}^{+}$IEL have been shown to mediate spontaneous NK cell like killing, possibly through surface NK cell markers (Guy-Grand et al., 1996). In humans, NK cell marker CD56 and NKG2D have been found on $\gamma \delta \mathrm{TCR}^{+} \mathrm{IEL}$, although human IEL do not appear to mediate much spontaneous ex vivo cytotoxicity (Deusch et al., 1991;Bauer et al., 1999) without stimulation by IL-2, IL-7 or IL-15, which induce so-called lymphokine activated killer (LAK) function (Ebert, 1999). Thus, $\mathrm{CD} 8 \alpha \alpha^{+} \alpha \beta \mathrm{TCR}^{+} \mathrm{IEL}$ and $\gamma \delta \mathrm{TCR}^{+} \mathrm{IEL}$ seem to remain cytolytically inactive until stimulated by a battery of IEC and LP cytokines and epithelial stress markers, thereby fine-tuning an otherwise non-specific response specifically against stressed or infected epithelial cells, possibly an ancient immune function predating the evolution of antigen-specific adaptive immunity (Hayday, 2000).

In addition to cytolysis of infected and possibly stressed epithelial cells (Suzuki et al., 1997), IEL influence epithelial barrier integrity. IEL have been shown to produce epithelial cell mitogen keratinocyte growth factor (KGF) that, in addition to KGF produced by LP fibroblasts, can repair damaged epithelium at the resolution of an inflammatory immune response. Indeed, mice lacking $\gamma \delta \mathrm{TCR}^{+}$IEL show a reduction in epithelial cell turnover (Komano et al., 1995), and resolution of mucosal inflammation with subsequent restoration of the epithelium is greatly impaired (Roberts et al., 1996). IEL are reciprocally influenced by the epithelial cell cytokine 
products, such as IEC IL-15 (Yoshikai et al., 1999) and goblet cell IL-7, which can induce IEL proliferation and LAK function, and IEC TGF- $\beta$ (Ebert, 1999) and nitric oxide (Chung et al., 2000), which have been shown to prevent IEL activation. Thus, as the immediate front line of the immune system in the intestine, different IEL subpopulations function to maintain normal epithelium growth homeostasis, non-specifically prevent infection of the epithelium, specifically resolve infection and guard against re-infection, and to resolve inflammation and repair epithelial damage, under the dynamic reciprocal control of numerous in situ factors of immune, epithelial, and stromal cell origin.

\section{Humoral Immunity:}

The primary follicles, located within the PP, appendix, or as dispersed isolated lymphoid aggregates, are the sites of initiation of humoral immune responses, and appear as roughly spherical lymphocyte aggregates containing predominately naive B cells that express several surface markers including $\mathrm{CD}_{19^{+}}, \mathrm{CD} 20^{+}$, surface $\operatorname{IgD}\left(\operatorname{sIgD}^{+}\right)$and $\operatorname{IgM}\left(\operatorname{sIgM}^{+}\right)($Farstad et. al., 2000). In addition, follicles contain follicular dendritic cells (FDC) and occasional $\mathrm{CD}^{+} \mathrm{Th}$ cells. Primary follicles initially occur in the developing fetal intestine, but the development of secondary follicles during the B cell proliferative event known as the germinal center (GC) reaction does not occur until after birth, concomitant with the establishment of gut microflora (Brandtzaeg et al., 1987). Naïve antigen specific B cells initially contact antigen percolating through the parafollicular $\mathrm{T}$ cell area by means of their antigen-binding surface $\operatorname{IgD}$ and $\operatorname{IgM}$, and subsequently internalize, process, and present epitopes to antigen-specific $\mathrm{CD} 4^{+} \mathrm{T}$ cells that themselves have been previously activated by the interdigitating dendritic cells in the parafollicular T cell area. In a manner that is analogous to the interaction between dendritic APC and T-cells, $\mathrm{CD}^{+}{ }^{+} \mathrm{T}$ cell CD40L interaction with $\mathrm{B}$ cell surface $\mathrm{CD} 40$ induces upregulation co- 
stimulatory B7 molecules on the B-cell, which binds T cell surface CD28 and induce effector cytokine production, which subsequently induces B cell proliferation, resulting in the germinal center (GC) reaction (Gulbranson-Judge et al., 1997; Maclennan et al., 1997). GC reactions occurring within extant primary follicles produce a characteristic appearance of the secondary follicle, with a dark zone of rapidly proliferating $\mathrm{Ki}-67^{+} \operatorname{sIgD}^{-} \operatorname{sIgM}^{+} \mathrm{CD} 38^{+}$centroblasts, which undergo isotype switching predominately to the IgA1 and IgA2 subclasses, and somatic hypermutation of antigen-binding heavy chain complementarity determining regions, a light zone of $\operatorname{sgD}^{-} \operatorname{sIgM}^{+} \mathrm{CD} 38^{+}$centrocytes, and a mantle zone of predominantly naïve B cells (Brandtzaeg and Bjerke, 1990). Low affinity antigen specific antibody produced early in the GC reaction, or from previous humoral responses forms insoluble antigen-antibody immune complexes on the membrane of FDC within the follicle. During a GC reaction, FDC are induced to shed these immune complexes on membrane vesicles known as immune complex coated bodies (Ahmed and Gray, 1996). Centrocytes with hypermutated heavy chains are competitively positively selected by their ability to bind and internalize these immune complexes, and subsequently to present epitopes to follicle-infiltrating $\mathrm{CD}^{+} \mathrm{T}$ cells, and thereby avoid apoptosis. This competitive selection for B cells expressing mutated surface immunoglobulin best able to bind limiting amounts of antigen is the basis of the phenomenon of affinity maturation, and allows for progressively more effective fine tuning of humoral immunity.

Following proliferation of activated antigen-specific B cell, somatic hypermutation and possibly isotype class switching, the resulting centrocytes do not usually immediately terminally differentiate to antibody secreting plasma cells, but rather they migrate from the lymphoid tissue via the efferent lymphatics to traffic to effector tissues, or they enter the marginal zone around the periphery of the follicular mantle zones to become a quiescent $\operatorname{sigD}{ }^{-} \operatorname{sig} \mathrm{M}^{+}$or $\operatorname{sIg} \mathrm{A}^{+} \mathrm{CD} 20^{+}$ 
reserve pool of antigen experienced 'memory' cells (Dunn-Walters et al., 1996). These cells await subsequent re-exposure to antigen, whereupon they can provide a secondary 'recall' response that is more rapid and of greater magnitude than the primary response.

The primary immunoglobulin isotype produced by mucosal B cells is IgA. In humans, there are two subclasses, IgA1 and IgA2. Effector plasma cells in the intestinal LP secrete predominantly IgA1 in the proximal intestine, and IgA2 distally, although substantial amounts of both are produced at all sites along the length of the intestine (Brandtzaeg and Bjerke, 1990). Lesser quantities of B cells expressing immunoglobulin of other isotypes are also produced. In order of occurrence, these are $\operatorname{IgM}>\operatorname{IgG} 1>\operatorname{IgG} 2>\operatorname{IgG} 3 / \operatorname{IgG} 4>\operatorname{IgE}$. The determination of isotype is determined in the initial priming site. Indeed, antigen experienced B cells exiting the gut via the mesenteric lymph expressed $\operatorname{sg} A$, $\operatorname{sgM}$ or $\operatorname{sIgG}$ in a ratio of 5:1: approximately 0.5 (Farstad et al., 1997). Commitment to the IgA isotype, which contrasts with the PLN isotype class switch preference for IgG was shown to be due to intrinsic factors of the mucosal priming site microenvironment. Later in vitro studies demonstrated that PP DC and T cell co-cultures with mitogen stimulated B cells produce IgA. It is now known that the intestinal $\mathrm{CD} 4^{+} \mathrm{T}$ cell derived cytokine TGF- $\beta$, and the interaction of T cell, and possibly DC CD40L with CD40 on the surface of activated B cells induces the IgA isotype class switching within the intestinal secondary follicles. The exact mechanisms for induction of IgA class switching and its regulation are still being elucidated.

\section{Intestinal effector $B$ cells:}

The intestinal lamina propria, which contains approximately $10^{10}$ Ig-producing B cells and plasma cells per meter, totaling roughly $80 \%$ of the body total, is far and away the largest B cell organ of the body (Brandtzaeg et al., 1999). In the human gut, $\operatorname{IgA}^{+} \mathrm{B}$ cells expressing either 
the IgA1 or IgA2 subclasses predominate over all other isotypes, accounting for about $80 \%$ of B cells in the LP of the proximal jejunum (77\% IgA1, 23\% IgA2), increasing to $84 \%$ in the ileum (60\% IgA1, 40\% IgA2), and 90\% in the large intestine (36\% IgA1, 64\% IgA2) (Kett et al., 1986). The relative predominance $\operatorname{IgA} 2^{+} \mathrm{B}$ cells in the large intestine may be due to the abundance of bacterial products such as LPS, which tend to induce isotype class switching to IgA2 (Kett et al., 1986). $\operatorname{IgM}^{+}$cells show a substantial though decreasing representation from $18 \%$ of B cells in the jejunum to $6 \%$ in the large intestine (Kett et al., 1986). $\operatorname{IgG}^{+}$cells represent about $4 \%$ of all B cells throughout the intestine, with a subclass distribution of $>60 \% \operatorname{IgG} 1,20 \%$ IgG2, and $<10 \%$ IgG3 and IgG4 (Bjerke and Brandtzaeg, 1990). $\operatorname{IgE}^{+}$and $\operatorname{IgD}^{+} \mathrm{LP} B$ cells are infrequent (Brandtzaeg et al., 1987). Nearly all $\mathrm{LP} \mathrm{IgA}^{+}$and $\operatorname{IgM}^{+} \mathrm{B}$ cells, and $>80 \%$ of $\operatorname{IgG}^{+} \mathrm{B}$ cells also express the $15 \mathrm{kDa}$ J (joining) chain (Bjerke and Brandtzaeg, 1990), which aggregates intracellular IgA monomers into predominantly dimeric IgA, and occasional larger polymeric masses, and aggregates intracellular IgM into predominantly pentameric forms, and occasional hexameric forms collectively called polymeric IgA (pIgA) and pIgM, respectively (Brandtzaeg et al., 1999a). Monomeric IgG, by contrast, does not aggregate into polymers, and J chain expression by $\operatorname{IgG}^{+}$cells is believed to be a by-product of mucosal priming of these cells (Brandtzaeg et al., 1999b). J-chain dependent pIgA and pIgM secreted by LP plasma cells is bound by the polymeric Ig receptor (pIgR) on IEC, translocated, and released in association with the secretory component (SC) following cleavage of the pIgR on the lumenal face of the IEC (Brandtzaeg et al., 1999b). Large amounts of IgG are also secreted into the intestinal lumen, most likely by passive diffusion through paracellular junctions (Prigent-Delecourt et al., 1995), although some evidence exists that an FcyR expressed on small intestinal IEC and provides for transport from the lumen to the LP of maternal IgG in breast milk, serves as a bi-directional 
transporter, and can transport LP IgG onto the mucosal surface (Dickinson et al., 1999). Much of this IgG is probably derived from serum protein filtrate in the LP. Taken together, these findings suggest a not inconsequential role for $\operatorname{IgG}$ in addition to $\mathrm{pIgA}$ and $\mathrm{pIgM}$ in normal intestinal immunity.

\section{Intestinal humoral immunity:}

Intestinal immunity, mounted under constant challenge by commensal and pathogenic organisms, is further complicated by the requirement to tolerate a massive load of normal floral and dietary-derived antigens. Despite the vast antigenic challenge, and the apparent chronic activation of the GALT, it is striking that not only is the intestinal mucosal not chronically, massively inflamed, but that with only rare exceptions, immune responses to most innocuous antigens are apparently inhibited. Perhaps the most important mechanism of preventing damaging immune responses to normal floral and dietary antigens is to preclude their contact with intestinal lymphocytes. This function is partially mediated by the epithelial cell and mucus barriers, and also by secreted antibody of predominantly the IgA isotype, with some contribution of IgM and IgG. Coating of lumenal organisms and antigens by secretory IgA prevents epithelial invasion by pathogens and commensal opportunists, as well as attenuating uptake of lumenal antigens, processes collectively known as "immune exclusion" (Neutra, 1999). In the LP, pIgA is bound by the pIgR on the basolateral surface of the IEC. Receptor bound pIgA is subsequently translocated to the lumenal, apical IEC surface, and is there released by proteolytic cleavage of the pIgAR. In the lumen, pIgA prevents antigen and organism contact with the IEC. It is also believed that during the translocation event pIgA may bind to antigen or organisms previously internalized by the IEC and "carry" it back to the lumen. By contrast, although FAE lacks pIgR (Brandtzaeg and Bjerke, 1989), and therefore does not transport pIgA into the gut lumen, M cell 
Fc $\alpha \mathrm{R}$ binds antigen-bound secretory $\operatorname{IgA}$ in the lumen, translocating it and any associated antigen into the PP for subsequent immune priming (Neutra, 1999). Nevertheless, normal human GALT does not prime humoral responses to dietary antigen, as potent IgA responses, in contrast to $\operatorname{IgE}$ responses, against food antigens do not occur in humans except in cases of chronic intestinal inflammatory diseases (Husby et al., 2000). Indeed, intestinal IgA responses seem exquisitely restricted to commensal and pathogenic organisms and their structural constituents and secreted products. This is perhaps most clearly illustrated in studies using germ-free mice, wherein $\mathrm{GC}$ reactions are absent until colonization of the mucosa with gut microflora, monoassociation with segmented filamentous bacteria, or monoinfection with the model intestinal pathogen, reovirus. Interestingly, while PP and appendix-derived B cells have been demonstrated to provide IgA and IgM lymphocytes throughout the gut LP, studies in mice suggest that up to half of all gut $\mathrm{LP}_{\operatorname{IgA}}^{+}$cells are the progeny of an unconventional population of peritoneal cavity derived $\mathrm{CD}^{+} \mathrm{B}$ cells, also known as $\mathrm{B} 1 \mathrm{~B}$ cells, as distinguished from conventional bone-marrow derived GC-primed B2 cells (Murakami and Honjo, 1995). B1 B cells tend to produce IgM of low affinity to non-proteinaceous bacterial structural components, such as phosphatidylcholine and lipopolysaccharide, generally considered to be Th cell independent antigens. Nevertheless, under the influence of Th cell cytokines possibly from peritoneal cavity $\mathrm{CD}^{+} \mathrm{T}$ cell, or lamina propria $\mathrm{T}$ cells, or even possibly such novel factors as enteric nervous system neuropeptide vasoactive intestinal peptide (Boirivant et al., 1994), activated B1 cells can undergo isotype class switching to IgA. It is thought that B1 B cells represent ancient, innate 'natural antibody' B cell immunity that forms an initial, non-specific, high volume, low affinity component of immune exclusion. By contrast, conventional (B2) B cells form a highly selective, high affinity response to commensal and pathogenic organisms that 
manage to penetrate the B1 B cell pIgA line of defense (Bos et al., 1999). Interestingly, low affinity immunoglobulin from B1 B cells against some bacteria constituents, such as phosphatidylcholine-coated surface glycoproteins, can cross react with tissue self-antigens, possibly precipitating antibody-mediated autoimmunity, a phenomenon, which is currently under investigation (Murakami and Honjo, 1995).

\section{Migration of lymphocytes from priming to effector sites:}

Initiation of immune responses in the PP results in production of antigen-specific antibody and the appearance of activated T-cells at effector sites in the intestine as well as at other mucosal sites such as the respiratory tract, the mammary gland, and perhaps the genitourinary tract. This phenomenon has led investigators to describe a 'Common Mucosal Immune System' (CMIS). The cellular basis for this CMIS has been understood for almost 30 years. Immune cells activated in the PP preferentially migrate back to the intestine, but a fraction will distribute to other mucosal compartments. The phenomenon of shared memory lymphocytes among anatomically distinct mucosal compartments is the rationale behind mucosal vaccine strategies aimed at providing protective immunity at targeted mucosal tissues by antigen application at physically more accessible and convenient locations. The molecular basis for this preferential re-circulation has been more clearly elucidated only in the last decade.

Most cells leaving the intestine and entering the mesenteric lymphatic vessels are believed to migrate to the mesenteric lymph nodes (MLN). Proliferating T and B cells from the GALT can produce large numbers of progeny in the MLN, which, along with quiescent recirculating naïve and memory cells, can reenter the efferent lymphatics, going on to the thoracic duct and then the systemic venous circulation. Antigen experienced T and B cells can then migrate to mucosal effector sites or to other secondary lymphoid tissues. 
Memory T and B cells exiting the gut express a heterodimeric protein receptor referred to as $\alpha_{4} \beta_{7}$ integrin, which allows for the preferential trafficking of gut-primed lymphocytes to the intestinal lamina propria and back to the intestinal priming tissues such as PP and MLN. The phenomenon of recirculation of antigen-experienced cells back to the gut tissues is mediated by $\alpha_{4} \beta_{7}$ ligation of the mucosal addresin cell adhesion molecule 1 (MadCAM-1) constitutively expressed on the intravascular surfaces of the flat endothelial cells of the post-capillary venules of the gut LP, and also on the specialized cuboidal high endothelial vessels (HEV) cells that serve as the points of egress of naïve and memory $\mathrm{T}$ and $\mathrm{B}$ cells from the venous circulation into the organized intestinal priming lymphoid tissues (Farstad et al., 1996). Differential glycosylation of the MadCAM molecule within the endothelial cells at these locations allow for specific interactions with distinct lymphocyte ligands expressed on naïve or activated lymphocytes. Thus, naïve cells are excluded from effector compartments, which preferentially recruit antigen experienced $\alpha_{4} \beta_{7}{ }^{+}$ cells.

Expression of $\alpha_{4} \beta_{7}$ is not confined solely to intestinally primed lymphocytes, or even more generally to mucosally primed lymphocytes. Nevertheless, cells primed in distinct mucosal immune compartments show a definite preference for returning to their compartment of priming. This is likely mediated by differential levels of expression of other integrins and chemokine receptors that contribute to lymphocyte homing. However, a detailed discussion of these interactions is beyond the scope of this chapter.

\section{Oral tolerance:}

Secretory $\operatorname{IgA}$, and to a lesser extent IgM mediated immune exclusion and epithelial barrier function to exclude lumenal antigen from the intestinal tissues are important mechanisms by which the intestine prevents uncontrolled immune activation and inflammation. Intestinal $\mathrm{T}$ 
cell responses, and by extension systemic $\mathrm{T}$ cell responses are tightly controlled by mechanisms ranging from outright elimination of antigen activated $\mathrm{T}$ cells, known as clonal deletion, debilitation of proliferation and effector function, known as anergy, and the generation of antigen specific effector $\mathrm{T}$ cells secreting a cytokine profile that attenuates other $\mathrm{T}$ and $\mathrm{B}$ cell responses, known as active suppression. Collectively, these mechanisms contribute to a phenomenon of elicited antigen-specific hyporesponsiveness, known as oral tolerance. Oral $\mathrm{T}$ cell tolerance is generated to protein antigens, and therefore requires T cell interaction with APC and antigen. The most important determinants of $\mathrm{T}$ cell tolerance as regards the mechanisms of clonal deletion or anergy are most likely a large quantity of antigen (Friedman and Meiner, 1994 ) and low levels of co-stimulatory molecule expression on APC presenting antigen to T cells (Van Gool et al., 1999; Koenen and Joosten, 2000). Both T cell recognition of antigen via the TCR, and CD28 ligation by APC B7 are required to activate naïve T cells. In the absence of sufficient APC activation by CD40L-CD40 interaction (Van Gool et al., 1999) or local inflammatory cytokines such as IL-12 (Villegas et al., 1999), the APC will express B7 at levels insufficient for naïve $T$ cell co-stimulation, rendering responder T cells anergic (Frauwirth et al., 2000). Antigen experienced T cells, which express the CD28-like high affinity receptor for B7, known as CTLA4, can be deleted by interaction with antigen on APC expressing limiting levels of B7, which preferentially binds CTLA-4 in lieu of CD28, thereby inducing T cell apoptosis (Perez et al., 1997). In instances where exceptionally high local concentration of antigen are present, the APC may provide such high numbers of MHC antigen complexes to induce responder T cells to undergo apoptosis, leading to tolerance by clonal deletion (Chen et al., 1997).

Active suppression, by contrast, is more complicated, involving under different circumstances $\mathrm{CD}^{+}, \mathrm{CD} 8^{+}$, or $\gamma \delta \mathrm{TCR}^{+} \mathrm{T}$ cells, and is dependent on CD40L-CD40 interaction 
and antigen presenting cell activation (Kweon et al., 1999). In general, active suppression is mediated by antigen-specific T cells that produce the pleiotropic cytokine TGF- $\beta$, and the Th2 associated cytokine IL-10 (Kitani et al., 2000). TGF- $\beta$, frequently expressed in GALT by CD4 ${ }^{+}$ Th3, or T regulatory cells either alone or in addition to a Th2 cytokine profile (Kitani et al., 2000), and also by some $\gamma \delta \mathrm{TCR}^{+}$IEL (Barrett et al., 1992) and IEC (Goodrich and McGee, 1998). TGF- $\beta$ strongly attenuates T cell responses (Levings and Roncarolo, 2000), while also serving as the primary B cell IgA switch factor (van Vlasselaer et al., 1992). Thus, TGF- $\beta$ serves to prevent damaging immunity directly, and by augmenting IgA mediated immune exclusion. IL10, a Th2 cytokine involved in downregulating APC antigen processing and presentation, and in blocking Th1 responses and cell mediated immune responses such as delayed type hypersensitivity, also contributes to the $\operatorname{IgA}$ response by promoting $\operatorname{IgA}^{+} \mathrm{B}$ cell differentiation to IgA secreting plasma cells. Indeed, IL-10 is secreted by IEC; possibly to both reduce APC presentation of cell-mediated immune responses to innocuous antigens, and to increase local pIgA production. GALT $\mathrm{CD} 8^{+} \mathrm{T}$ cells also mediate active suppressive tolerance by TGF- $\beta$ production, often in concert with TGF- $\beta$ producing $\mathrm{CD}^{+} \mathrm{T}$ cells. Animal studies have shown a pivotal role for $\gamma \delta \mathrm{TCR}^{+} \mathrm{T}$ cells in oral tolerance induction and maintenance, as oral tolerance to ovalbumin protein could not be established in mice depleted of $\gamma \delta \mathrm{TCR}^{+}$T cells (Mengel et al., 1995; Ke et al., 1997). Furthermore, previously established oral tolerance to ovalbumin protein could be abrogated by depletion in vivo of $\gamma \delta \mathrm{TCR}^{+} \mathrm{T}$ cells (Mengel et al., 1995). Conversely, adoptive transfer of murine $\gamma \delta \mathrm{TCR}^{+} \mathrm{T}$ cells has been shown to abrogate previously established oral tolerance (Fujihashi et al., 1992). The complex role of $\gamma \delta \mathrm{TCR}^{+} \mathrm{T}$ cells in oral tolerance induction and maintenance are subject to ongoing investigation. $\gamma \delta \mathrm{TCR}^{+} \mathrm{T}$ cells of the IEL compartment also have a crucial supportive role in lamina propria IgA production, as TCR $\delta$ - 
chain gene deleted mice have severely diminished LP IgA production, but normal LP IgM and IgG production, suggesting an important role of mucosal $\gamma \delta \mathrm{TCR}^{+} \mathrm{T}$ cells in maintaining $\operatorname{IgA}$ immune exclusion. Numerous clinical studies have been initiated attempting to ameliorate intestinal and systemic immunopathology by inducing tolerogenic intestinal immune responses, thus exploiting a natural protective phenomenon for broad therapeutic benefit. 


\section{References}

Abuzakouk M, Kelleher D, Feighery C, O’Farrelly C. Increased HLA-DR and decreased CD3 on human intestinal intraepithelial lymphocytes; evidence of activation. Gut 1996;39:396-400.

Abuzakouk M, Carton J, Feighery C, O’Donoghue DP, Weir DG, O’Farrelly C. CD4+ CD8+ and CD8alpha+beta- T lymphocytes in human small intestinal lamina propria. Eur. $J$. Gastroenterol. Hepatol. 1998;10:325-329.

Ahmed R, Gray D. Immunological memory and protective immunity: understanding their relation. Science 1996;272:54-60.

Arstila T, Arstila TP, Calbo S, Selz F, Malassis-Seris M, Vassalli P, Kourilsky P, Guy-Grand D. Identical $\mathrm{T}$ cell clones are located in the mouse gut epithelium and lamina propria and circulate in the thoracic duct lymph. J. Exp. Med. 2000;191:823-834.

Bauer S, Groh V, Wu J, Steinle A, Phillips JH, Lanier LL, Spies T. Activation of NK cells and T cells by NKG2D, a receptor for stress-inducible MICA. Science 1999;285:727-729.

Barrett TA, Gajewski TF, Danielpour D, Chang EB, Beagley KW, Bluestone JA. Differential function of intestinal intraepithelial lymphocyte subsets. J. Immunol. 1992;149:1124-1130. 
Beagley KW, Fujihashi K, Black CA, Lagoo AS, Yamamoto M, McGhee JR, Kiyono H. The Mycobacterium tuberculosis 71-kDa heat-shock protein induces proliferation and cytokine secretion by murine gut intraepithelial lymphocytes. Eur. J. Immunol. 1993;23:2049-2052.

Biron CA. Cytokines in the generation of the immune responses to, and resolution of, virus infection. Curr. Opin. Immunol. 1994;6:530-538.

Bjerke K, Brandtzaeg P. Terminally differentiated human intestinal B cells. J chain expression of IgA and IgG subclass-producing immunocytes in the distal ileum compared with mesenteric and peripheral lymph nodes. Clin. Exp. Immunol. 1990;82:411-415.

Bjerke K, Brandtzaeg P. Terminally differentiated human intestinal B cells. IgA and IgG subclass-producing immunocytes in the distal ileum, including Peyer's patches, compared with lymph nodes and palatine tonsils. Scand. J. Immunol. 1990;32:61-67.

Boirivant M, Fais S, Annibale B, Agostini D, Delle Fave G, Pallone F. Vasoactive intestinal polypeptide modulates the in vitro immunoglobulin A production by intestinal lamina propria lymphocytes. Gastroenterology 1994;106:576-582.

Boirivant M, Fuss I, Fiocchi C, Klein JS, Strong SA, Strober W. Hypoproliferative human lamina propria $\mathrm{T}$ cells retain the capacity to secrete lymphokines when stimulated via CD2/CD28 pathways. Proc. Assoc. Am. Physicians 1996;108:55-67. 
Bos NA, Cebra JJ, Kroese FGM. B-1 cells and the intestinal microflora. Curr Top. Microbiol. Immunol. 2000;252:211-220.

Brandtzaeg P, Baekkevold ES, Farstad IN, Jahnsen FL, Johansen FE, Nilsen EM, Yamanaka T. Regional specialization in the mucosal immune system: what happens in the microcompartments? Immunol Today 1999;20:141-151.

Brandtzaeg P, Bjerke K, Kett K, Kvale D, Rognum TO, Scott H, Sollid LM, Valnes K. Production and secretion of immunoglobulins in the gastrointestinal tract. Ann. Allergy 1987;59:21-39.

Brandtzaeg P, Bjerke K. Human Peyer's patches: Lympho-epithelial relationships and characteristics of immunoglobulin-producing cells. Immunol. Invest. 1989;18:29-45.

Brandtzaeg P, Bjerke K. Immunomorphological characteristics of human Peyer's patches. Digestion 1990;2: 262-273.

Braunstein J, Qiao L, Autschbach F, Schurmann G, Meuer S. T cells of the human intestinal lamina propria are high producers of interleukin-10. Gut 1997;41:215-220.

Burdin N, Van Kooten C, Galibert L, Abrams JS, Wijdenes J, Banchereau J, Rousset F. Endogenous IL-6 and IL-10 contribute to the differentiation of CD40-activated human B lymphocytes. J. Immunol. 1995;154: 2533-2544. 
Chardes T, Buzoni-Gatel D, Lepage A, Bernard F, Bout D. Toxoplasma gondii oral infection induces specific cytotoxic CD8 alpha/beta+ Thy-1+ gut intraepithelial lymphocytes, lytic for parasite-infected enterocytes. J. Immunol. 1994;153:4596-4603.

Chen Y, Inobe J, Weiner HL. Inductive events in oral tolerance in the TCR transgenic adoptive transfer model. Cell. Immunol. 1997;178:62-68.

Chen D, Lee F, Cebra JJ, Rubin DH. Predominant T cell receptor Vbeta usage of intraepithelial lymphocytes during the immune response to enteric reovirus infection. J. Virol. 1997;71:34313436.

Chung CS, Song GY, Wang W, Chaudry IH., Ayala A. Septic mucosal intraepithelial lymphoid immune suppression: role for nitric oxide not Interleukin-10 or transforming growth factor-beta. J. Trauma. 2000;48:807-812.

Corazza N, Muller S, Brunner T, Kagi D, Mueller C. Differential contribution of Fas- and perforin -mediated mechanisms to the cell-mediated cytotoxic activity of naïve and in vivo primed intestinal intraepithelial lymphocytes. J. Immunol. 2000;164:398-403.

Cuff CF, Cebra CK, Rubin DH, Cebra JJ. Developmental relationship between cytotoxic alpha/beta T cell receptor-positive intraepithelial lymphocytes and Peyer's patch lymphocytes. Eur. J. Immunol. 1993;23:1333-1339. 
Danis VA, Heatly RV. Evidence for regulation of human colonic mucosal immunoglobulin secretion by intestinal lymphoid cells. J. Clin. Lab. Immunol. 1987;22:7-11.

Dasso JF, Obiakor H, Bach H, Anderson AO, Mage RG. A morphological and immunohistological study of the human and rabbit appendix for comparison with the avian bursa. Dev. Comp. Immunol. 2000;24:797-814.

De Maria R, Boirivant M, Cifone MG, Ronciaoli P, Hahne M, Tschopp J, Pallone F, Santoni A, Testi R. Functional expression of Fas and Fas ligand on human gut lamina propria $\mathrm{T}$ lymphocytes. A potential role for the acidic sphingomyelinase pathway in normal immunoregulation. J. Clin. Invest. 1996;97,316-322.

De Maria R, Fais S, Silvestri M, Frati L, Pallone F, Santoni A, Testi R. Continuous in vivo activation and transient hyporesponsiveness to $\mathrm{TcR} / \mathrm{CD} 3$ triggering of human gut lamina propria lymphocytes. Eur. J. Immunol. 1993;23:3104-3108.

Deusch K, Pfeffer K, Reich K, Gestettenbauer M., Daum S, Luling F, Classes M. Phenotypic and functional characterization of human TCR gamma delta+ intestinal intraepithelial lymphocytes. Curr. Top. Microbiol. Immunol. 1991;173:279-283. 
Deusch K, Luling F, Reich K, Classes M, Wagner H, Pfeffer K. A major fraction of human intraepithelial lymphocytes simultaneously expresses the gamma/delta T cell receptor, the CD8 accessory molecule and preferentially uses the $\mathrm{V}$ delta 1 gene segment. Eur. J. Immunol. 1991;21:1053-1059.

Dickinson BL, Badizadegan K, Wu Z, Ahouse JC, Zhu X, Simister NE, Blumberg RS, Lencer WI. Bidirectional FcRn-dependent IgG transport in a polarized human intestinal epithelial cell line. J. Clin. Invest. 1999;104:903-911.

Dunkley ML, Husband AJ. Distribution and functional characteristics of antigen-specific helper T cells arising after Peyer's patch immunization. Immunology 1987;61:475-482.

Dunn-Walters DK, Isaacson PG, Spencer J. Sequence analysis of rearranged IgVH genes from microdissected human Peyer's patch marginal zone B cells. Immunology 1996;88:618-624.

Ebert EC, Roberts AI. Costimulation of the CD3 pathway by CD28 ligation in the human intestinal lymphocytes. Cell Immunol. 1996;171:211-216.

Ebert EC. Tumor necrosis factor-alpha enhances intraepithelial lymphocyte proliferation and migration. Gut 1998;42:650-655.

Ebert EC. Inhibitory effects of transforming growth factor-beta (TGF-beta) on certain functions of intraepithelial lymphocytes. Clin. Exp. Immunol. 1999;115:415-420. 
Fan JY, Boyce CS, Cuff CF. T-helper 1 and T-helper 2 cytokine responses in gut-associated lymphoid tissue following enteric reovirus infection. Cell. Immunol. 1998;188:55-63.

Farrar MA, Screiber RD. The molecular cell biology of interferon-gamma and its receptor. Annu. Rev. Immunol. 1993;11:571-611.

Farstad IN, Carlsen H, Morton HC, Brandtzaeg P. Immunoglobulin A cell distribution in the human small intestine: phenotypic and functional characteristics. Immunology 2000;101:354-363.

Farstad IN, Halstensen TS, Fausa O, Brandtzaeg P. Heterogeneity of M-cell-associated B and T cells in human Peyer's patches. Immunology 1994;83:457-464.

Farstad IN, Halstensen TS, Lien B, Kilshaw PJ, Lazarovitz AI. Distribution of b7 integrins in the human intestinal mucosal and organized gut-associated lymphoid tissue. Immunology 1996;89:227-237.

Farstad IN, Norstein J, Brandtzaeg P. Phenotypes of T and B cells in human intestinal and mesenteric lymph. Gastroenterology 1997;112:163-173.

Frauwirth KA, Alegre ML, Thompson CB. Induction of T cell anergy in the absence of CTLA4/B7 interaction. J. Immunol. 2000;164:2987-2993. 
Friedman A, Weiner HL. Induction of anergy or active suppression following oral tolerance is determined by antigen dosage. Proc. Natl. Acad. Sci. USA 1994;91:6688-6692.

Fujihashi K, Taguchi T, Aicher WK, McGhee JR, Bluestone JA, Eldridge JH, Kiyono H. Immunoregulatory functions for murine intraepithelial lymphocytes: gamma/delta $\mathrm{T}$ cell receptor-positive $(\mathrm{TCR}+) \mathrm{T}$ cells abrogate oral tolerance, while alpha/beta $\mathrm{TCR}+\mathrm{T}$ cells provide B cell help. J. Exp. Med. 1992;175:695-707.

Gebbers JO, Kennel I, Laissue JA. Lymphoid follicles of the human large bowel mucosal: structure and function. Verh. Dtsch. Ges. Pathol. 1992;76:126-130.

Gonsky R, Deem RL, Bream JH, Lee DH, Young HA, Targan SR. Mucosa-specific targets for regulation of IFN-gamma expression: lamina propria $\mathrm{T}$ cells use different cis-elements than peripheral blood T cells to regulate transactivation of IFN-gamma expression. J. Immunol. 2000;164:1399-1407.

Gonsky R, Deem RL, Hughes CC, Targan SR. Activation of the CD2 pathway in lamina propria T cells up-regulates functionally active AP-1 binding to the IL-2 promoter, resulting in messenger RNA transcription and IL-2 secretion. J. Immunol. 1998;160:4914-4922.

Goodrich ME, McGee DW. Regulation of mucosal B cell immunoglobulin secretion by intestinal epithelial cell-derived cytokines. Cytokine 1998;10:948-955. 
Griffith E, Ramsburg E, Hayday A. Recognition of human gut gamma-delta cells of stressinducible major histocompatibility molecules on enterocytes. Gut 1998;43:166-167.

Groh V, Steinle A, Bauer S, Spies T. Recognition of stress-induced MHC molecules by intestinal epithelial gamm delta T cells. Science 1998;279:1737-1740.

Grutzkau A, Hanski C, Hahn H, Riecken EO. Involvement of M cells in the bacterial invasion of Peyer's patches: a common mechanism shared by Yersinia entercolitica and other enteroinvasive bacteria. Gut 1990;31:1011-1015.

Gulbranson-Judge A, Casamayor-Palleja M, MacLennan IC. Mutually dependent T and B cell responses in germinal centers. Ann N.Y. Acad. Sci. 1997:815:199-210.

Guy-grand D, Cuenod-Jabri B, Malassis-Seris M, Selz F, Vassalli P. Complexity of the mouse gut $\mathrm{T}$ cell immune system: identification of two distinct natural killer $\mathrm{T}$ cell intraepithelial lineages. Eur. J. Immunol. 1996;26:2248-2256.

Hayday AC. [gamma][delta] cells: a right time and a right place for a conserved third way of protection. Аnпи. Rev. Immunol. 2000;18:975-1026.

Higgins JM, Mandlebrot DA, Shaw SK, Russell GJ, Murphy EA, Chen YT, Nelson WJ, Parker CM, Brenner MB. Direct and regulated interaction of integrin alphaEbeta7 with E-cadherin. $J$. Cell Biol. 1998;140:197-210. 
Hiroi T, Yanagita M, Ohta N, Sakaue G, Kiyono H. IL-15 and IL-15 receptor selectively regulate differentiation of common mucosal immune system-independent B-1 cells for IgA responses. $J$. Immunol. 2000;165:4329-4337.

Hoang P, Crotty B, Dalton HR, Jewell DP. Epithelial cells bearing class II molecules stimulate allogeneic human colonic intraepithelial lymphocytes. Gut 1992;33:1089-1093.

Husby, S. Normal immune responses to ingested foods. J. Pediatr. Gastroenterol. Nutr. 2000. 30suppl:S13-19.

Jabbal I, Kells DI, Forstner G, Forstner J. Human intestinal goblet cell mucin. Can. J. Biochem. 1979;54:707-716.

Jacob E, Baker SJ, Swaminathan SP. 'M' cells in the follicle-associated epithelium of the human colon. Histopathology 1987;11:941-952.

Jarry A, Cerf-Bensussan N, Brousse N, Selz F, Guy-Grand D. Subsets of CD3+ (T cell receptor alpha/beta or gamma/delta) and CD3- lymphocytes isolated from normal human gut epithelium display phenotypical features different from their counterparts in peripheral blood. Eur. J. Immunol. 1990;20:1097-1103.

Jones DE, Bevin CL. Paneth cells of the human small intestine express an antimicrobial peptide gene. J. Biol. Chem. 1992;267:23216-23225. 
Ke Y, Pearce K, Lake JP, Ziegler HK, Kapp JA. Gamma delta T lymphocytes regulate the induction and maintenance of oral tolerance. J. Immunol. 1997;158:3610-3618.

Kelsall BL, Strober W. The role of dendritic cells in antigen processing in the Peyer's patch. Ann. N.Y. Acad. Sci. 1996;778:47-54.

Kett K, Brandtzaeg P, Radl J, Haaijman JJ. Different subclass distribution of IgA-producing cells in human lymphoid organs and various secretory tissues. J. Immunol. 1986;136:3631-3635.

Kitani A, Chua K, Nakamura K, Strober W. Activated self-MHC-reactive T cells have the cytokine phenotype of the Th3/T regulatory cell 1 T cells. J. Immunol. 2000;165:691-702.

Kiyohara H, Egami H, Shibata Y, Murata K, Ohshima S, Ogawa M. Light microscopic immunohistochemical analysis of the distribution of groups II phospholipase A2 in human digestive organs. J. Histochem. Cytochem. 1992;40:1659-1664.

Kohbata S, Yokoyama H, Yabuuchi E. Cytopathogenic effect of Salmonella typhi GIFU 10007 on M cells of murine Peyer's patches in ligated ileal loops: an ultrastructural study. Microbiol. Immunol. 1986;30: 1225-1237.

Koenen HJ, Joosten I. Blockade of CD86 and CD40 induces alloantigen-specific immunoregulatory $\mathrm{T}$ cells that remain anergic even after reversal of hyporesponsiveness. Blood 2000;95:3153-3161. 
Komano H, Fujiura Y, Kawaguchi M, Matsumoto S, Hashimoto Y, Obana S, Mombaerts P, Tonegawa S, Yamamoto H, Itohara S. Homoestatic regulation of intestinal epithelia by intraepithelial gamma delta T cells. Proc. Natl Acad. Sci. USA 1995;92:6147-6151.

Kremer IB, Cooper KD, Teunissen MB, Stevens SR. Low expression of CD40 and B7 on macrophages infiltrating UV-exposed human skin; role in IL-2Ralpha-T cell activation. Eur. $J$. Immunol. 1998;28:2936-2946.

Kvale D, Krajci P, Brandtzaeg P. Expression and regulation of adhesion molecules ICAM-1 (CD54) and LFA-3 (CD58) in human intestinal epithelial cell lines. Scand. J. Immunol. 1992;35:669-676.

Kweon MN, Fujihashi K, Wakatsuki Y, Koga T, Yamamoto M, McGhee JR, Kiyono H. Mucosally induced systemic T cell unresponsiveness to ovalbumin requires CD40 ligand -CD40 interactions. J. Immunol. 1999;162:1904-1909.

Levings MK, Roncarolo MG. T-regulatory 1 cells: a novel subset of CD4 T cells with immunoregulatory properties. J. Allergy Clin. Immunol. 2000;106:S109-112.

Li Y, Yio XY, Mayer L. Human intestinal epithelial cell induced CD8+ T cell activation is mediated through CD8 and the activation of CD8-associated p561ck. J. Exp. Med. 1995;182:1079-1088. 
Lundqvist C, Melgar S, Yeung MM, Hammarstrom S, Hammarstrom ML. Intraepithelial lymphocytes in human gut have lytic potential and a cytokine profile that suggests $\mathrm{T}$ helper 1 and cytotoxic functions. J. Immunol. 1996;157:1926-1934.

MacDonald TT, Bajaj-Elliot M, Pender SLF. T cells orchestrate intestinal mucosal shape and integrity. Immunol. Today 1999;20:505-510.

MacLennan IC, Gulbranson-Judge A, Toellner KM, Casamayor-Palleja M, Chan E, Sze, DM, Luther SA, Orbea HA. The changing preference of T and B cells for partners as T-dependent antibody responses develop. Immunol. Rev. 1997:156:53-66.

Marcial MA, Madara JL. Cryptosporidium: cellular localization, structural analysis of absorptive cell parasite membrane-membrane interactions in guinea pigs, and suggestion of protozoan transport by M cells. Gastroenterology 1986;90:583-594.

Mattapallil JJ, Smit-McBride Z, McChesney M, Dandekar S. Intestinal intraepithelial lymphocytes are primed for gamma interferon and MIP-1beta expression and display antiviral cytotoxic activity despite severe CD4(+) T-cell depletion in primary simian immunodeficiency virus infection. J. Virol. 1998;72: 6421-6429.

McHugh S, Deighton J, Rifkin I, Ewan P. Kinetics and functional implication of Th1 and Th2 cytokine production following activation of peripheral blood mononuclear cells in primary culture. Eur. J. Immunol. 1996;26:1260-1265. 
McHugh S M, Rifkin I, Deighton J, Wilson AB, Lachmann PJ, Lookwood CM, Ewan P. The immunosuppressive drug thalidomide induces T helper cell type 2 (Th2) and concomitantly inhibits Th1 cytokine production in mitogen- and antigen-stimulated human peripheral blood mononuclear cell cultures. Clin. Exp. Immunol. 1995;99:160-167.

McLellan AD, Sorg RV, Williams LA, Hart DN. Human dendritic cells activate T lymphocytes via a CD40: CD40 ligand-dependent pathway. Eur. J. Immunol. 1996;26:1204-1210.

Mengel J, Cardillo F, Aroeira LS, Williams O, Russo M, Vaz, NM. Anti-gamma delta T cell antibody blocks the induction and maintenance of oral tolerance to ovalbumin in mice. Immunol. Lett. 1995;48:97-102.

Modlin IM, Poulsom R. Trefoil peptides: mitogens, motogens, or mirages? J. Clin. Gastroenterology. 1997;1:S94-100.

Moghaddami M, Cummins A, Mayerhofr G. Lymphocyte-filled villi: comparisons with other lymphoid aggregations in the mucosal of the human small intestine. Gastroenterology $1998 ; 115: 1414-1425$.

Mowat AM, Viney JL. The anatomical basis of intestinal immunity. Immunol. Rev. $1997 ; 156: 145-166$. 
Murakami M, Honjo T. The involvement of B-1 cells in mucosal immunity and autoimmunity. Immunol. Today 1995;16:534-539.

Neutra MR, Pringault E, Kraehenbuhl JP. Antigen sampling across epithelial barriers and induction of mucosal immune responses. Аnпu. Rev. Immunol. 1996;14:275-300.

Neutra MR. M cells in antigen sampling in mucosal tissues. Curr. Top. Microbiol. Immunol. $1999 ; 236: 17-32$.

Panja A, Blumberg RS, Balk SP, Mayer L. CD1d is involved in T cell-intestinal epithelial cell interactions. J. Exp. Med. 1993;178:1115-1119.

Perez VL, Van Parijs L, Biuckians A, Zheng XX, Strom TB, Abbas AK. Induction of peripheral T cell tolerance in vivo requires CTLA-4 engagement. Immunity 1997;4:11-417.

Petro TM, Chen SS, Panther RB. Effect of CD80 and CD86 on T cell cytokine production. Immunol. Invest. 1995;4:65-976.

Pitman RS, Blumberg RS. First line of defense: the role of the intestinal epithelium as an active component of the mucosal immune system. Gastroenterology 2000;35:805-814.

Pluschke G, Taube H, Krawinkel U, Pfeffer K, Wagner H, Classes M, Deusch K. Oligoclonality and skewed $\mathrm{T}$ cell receptor $\mathrm{V}$ beta gene segment expression in in vivo activated human intestinal intraepithelial T lymphocytes. Immunobiology 1994;192:77-93. 
Podolsky DK, Lynch-Devaney K, Stow JL, Oates P, Murgue B, De-Beaumont M, Sands BE, Mahida YR. Identification of human intestinal trefoil factor. Goblet cell-specific expression of a peptide targeted for apical secretion. J. Biol. Chem. 1993;268:6694-6702.

Podolsky DK. Mechanisms of regulatory peptide action in the gastrointestinal tract: trefoil peptides. Gastroenterology 2000;35:69-74.

Prigent-Delecourt L, Coffin B, Colombel JF, Dehinnin JP, Vaerman JP, Raumbaud JC. Secretion of immunoglobulins and plasma proteins from the colonic mucosal: an in vivo study in man. Clin. Exp. Immunol. 1995;99:221-225.

Regnault A, Kourilsky P, Cumano A. The TCR-beta chain repertoire of gut-derived T lymphocytes. Semin. Immunol. 1995;7:307-319.

Riordan SM, McIver CJ, Wakefield D, Thomas MC, Duncombe VM, Bolin TD. Interleukin-6 and small intestinal luminal immunoglobulins. Dig. Dis. Sci. 1998;43:442-445.

Roberts AI. Nadler SC., Ebert EC. Mesenchymal cells stimulate human intestinal intraepithelial lymphocytes. Gastroenterology 1997;113:144-150.

Roberts SJ, Smith AJ, West AB, Wen L, Findly RC, Owen MJ, Hayday AC. T-cell alpha beta+ and gamma delta + deficient mice display abnormal but distinct phenotypes toward a natural, widespread infection of the intestinal epithelium. Proc. Natl. Acad. Sci. USA 1996;93:1177411779. 
Rousset F, Garcia E, Banchereau J. Cytokine-induced proliferation and immunoglobulin production of human B lymphocytes triggered through their CD40 antigen. J. Exp. Med. 1991;173:705-710.

Schieferdecker HL, Ullrich R, Hirseland H, Zeitz M. T cell differentiation antigens on lymphocytes in the human intestinal lamina propria. J. Immunol. 1992;149:2816-2822.

Schmitz J, Assenmacher M, Radbruch A. Regulation of T helper cell cytokine expression: functional dichotomy of antigen-presenting cells. Eur. J. Immunol. 1993;23:191-199.

Shibuya K, Robinson D, Zonin F, Hartley SB, Macatonia SE, Somoza C, Hunter CA, Murphy KM, O'Garra A. IL-1 alpha and TNF-alpha are required for IL-12-induced development of Th1 cells producing high levels of IFN-gamma in BALB/C but not C57BL/6 mice. J. Immunol. 1998;160:1708-1716.

Sicinski P, Rowinski J, Warchol JB, Jarzabek Z, Gut W, Szcygiel B, Bielecki K, Koch G. Poliovirus type 1 enters the human host through intestinal M cells. Gastroenterology 1990;98:56-58.

Steinman RM, Pack M, Inaba K. Dendritic cells in the T-cell areas of lymphoid organs. Immunol. Rev. 1997;156:25-37. 
Suzuki Y, Mori K, Iwanaga T. Intraepithelial gamma delta T cells are closely associated with apoptotic enterocytes in the bovine intestine. Arch. Histol. Cytol. 1997;60:319-328.

Sydora BC, Brossay L, Hagenbaugh A, Kronenberg M, Cheroutre H. TAP-independent selection of CD8+ intestinal intraepithelial lymphocytes. J. Immunol. 1996;56:4209-4216.

Sydora BC, Jamieson BD, Ahmed R, Kronenberg M. Intestinal intraepithelial lymphocytes respond to systemic lymphocytic choriomeningitis virus infection. Cell. Immunol. 1996;167:161169.

Targan SR, Deem RL, Liu M, Wang S, Nel A. Definition of a lamina propria T cell responsive state. Enhanced cytokine responsiveness of T cells stimulated through the CD2 pathway. J. Immunol. 1995;154:664-675.

Trejdosiewicz LK, Smart CJ, Oakes DJ, Howdle PD, Malizia G, Campana D, Boylston AW. Expression of T-cell receptors TcR1 (gamma/delta) and TcR2 (alpha/beta) in the human intestinal mucosal. Immunology 1989;68:7-12.

Ullrich R, Schieferdecker HL, Ziegler K, Riecken EO, Zeitz M. gamma delta T cells in the human intestine express surface markers of activation and are preferentially located in the epithelium. Cell Immunol. 1990;128:619-627. 
Valnes K, Brandtzaeg P, Elgio K, Stave R. Specific and nonspecific humoral defense factors in the peithelium of normal and inflamed gastric mucosal. Immunohistochemical localization of immunoglobulins, secretory component, lysozyme, and lactoferrin. Gastroenterology $1984 ; 86: 402-412$.

Van Gool SW, Vermeiren J, Rafiq K, Lorr K, de Boer M, Ceuppens JL. Blocking of CD40CD154 and CD80/CD86-CD28 interactions during primary allogeneic stimulation results in T cell anergy and high IL-10 production. Eur. J. Immunol. 1999;29:2367-2375.

Van Houten N, Mixter PF, Wolfe J, Budd, RC. CD2 expression on murine intestinal intraepithelial lymphocytes is bimodal and defines proliferative capacity. Int. Immunol. $1993 ; 5: 665-672$.

Van Vlasselaer P, Punnonen J, de Vries JE. Transforming growth factor-beta directs IgA switching in human B cells. J. Immunol. 1992;148:2062-2067.

Villegas EN, Elloso MM, Reichmann G, Peach R, Hunter CA. Role of CD28 in the generation of effector and memory responses required for resistance to Toxoplasma gondii. J. Immunol. $1999 ; 163: 3344-3353$.

Wassef JS, Keren DF, Mailloux JL. Role of M cells in initial antigen uptake and in ulcer formation in the rabbit intestinal loop model of shigellosis. Infect. Immun. 1989;57:858- 863. 
Watanabe M, Ueno Y, Yajima T, Iwao Y, Tsuchiya M, Ishikawa H, Aiso S, Hibi T, Ishii H. Interleukin 7 is produced by human intestinal epithelial cells and regulates the prolferation of intestinal mucosal lymphocytes. J. Clin. Invest. 1995;95:2945-2953.

Wolfe JL, Rubin DH, Finberg R, Kauffman RS, Sharpe AH, Trier JS, Fields BN. Intestinal M cells: a pathway for entry of reovirus into the host. Science 1983;212:471-472.

Yoshikai Y. The interaction of intestinal epithelial cells and intraepithelial lymphocytes in host defense. Immunol. Res. 1999;20:219-235.

Zeitz M, Greene WC, Peffer NJ, James SP. Lymphocytes isolated from the intestinal lamina propria of normal nonhuman primates have increased expression of genes associated with T-cell activation. Gastroenterology 1988;94:647-655. 


\title{
Curriculum Vitae
}

\author{
Jonathan Reid Fulton
}

\section{Educational Background:}

B.S., Biology. University of Pittsburgh, Pittsburgh PA, 1993

Post-Bacc. Studies, CCAC, Pittsburgh PA, 1993-1994.

M.D. West Virginia University School of Medicine, Morgantown WV, 2004

Ph.D., Immunology. West Virginia University School of Medicine, Morgantown WV, 2004

\section{Work Experience:}

Graduate Teaching Asst. West Virginia University Dept. Microbiology and Immunology. 19962001.

Research Asst. Department of Immunopathology, Children's Hospital, UPMC, Oakland PA, 1992-1994.

\section{Publications:}

- Fulton JR, Cuff CF. Effects of Aging on Humoral and Cellular Immune Responses to Reovirus. Manuscript submitted for review. 2004.

- Fulton JR, Smith J, Cunningham C, Cuff CF. Influence of the Route of Infection on the Development of T-Cell Receptor Beta-Chain Repertoires of Reovirus-Specific Cytotoxic T Lymphocytes. J. Virol. 2004 Feb; 78(3): 1582-90.

- Fulton JR, Cunningham C, Cuff CF. Intestinal Immunity. Colonic Diseases, Humana Press. 2003.

- Cunnigham C, Fulton JR, Cuff CF. Inflammation in Intestinal Disease. Colonic Diseases. Humana Press. 2003.

- Cuff CF, Fulton JR, Barnett JB, Boyce CS. Enteric Reovirus Infection as a Probe to Study Immunotoxicity of the Gastrointestinal Tract. Toxicol. Sci. 1998 Apr; 42(2):99108.

- Moyna NM, Acker GR, Fulton JR, Weber K, Goss FL, Robertson RJ, Tollerud DJ, Rabin BS. Lymphocyte Fuction and Cytokine Production During Incremental Exercise in Active and Sedentary Males and Females. Int. J. Sports Med. 1996 Nov; 17(8): 585-91. 
- Moyna NM, Acker GR, Fulton JR, Weber K, Goss FL, Robertson RJ, Rabin BS. Exercise-Induced Alterations in Natural Killer Cell Number and Function. Eur J. Appl Physiol Occup Physiol. 1996; 74(3): 227-33.

- Moyna NM, Acker GR, Fulton JR, Weber K, Goss FL, Robertson RJ, Rabin BS. The Effects of Incremental Submaximal Exercise on Circulating Leukocytes in Physically and Sedentary Males and Females. Eur. J. Appl. Physiol. Occup. Physiol. 1996; 74(3): $211-8$.

\section{Awards:}

Eagle Scout Award, 1988.

Phi Eta Sigma Freshman Honor Society, University of Pittsburgh, 1990.

Phi Beta Kappa Honor Society, University of Pittsburgh, 1991.

Golden Key National Honor Society, University of Pittsburgh, 1992.

Alpha Omega Alpha Medical Honor Society, West Virginia University School of Medicine, 2002. 